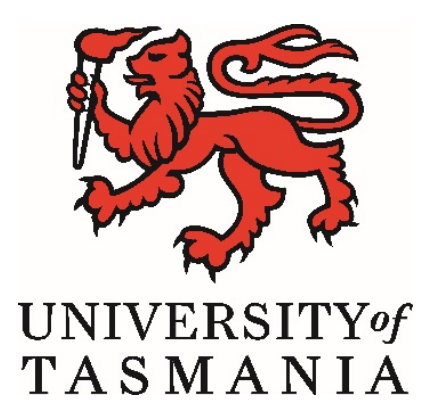

\title{
Power Management Optimization of Hybrid Power Systems for Electric Ferries
}

\author{
By \\ Monaaf Dheaa Abdulradha Al-Falahi \\ Australian Maritime College \\ College of Sciences and Engineering \\ University of Tasmania
}


[Page intentionally left blank] 


\section{Declarations}

\section{Declaration of Originality and Authority of Access}

I declare that this thesis contains no material which has been accepted for a degree or diploma by the University or any other institution, except by way of background information and duly acknowledged in the thesis, and that, to the best of my knowledge and belief, this thesis contains no material previously published or written by another person, except where due acknowledgement is made in the text of the thesis.

This thesis may be made available for loan and limited copying in accordance with the Copyright Act 1968.

\section{Statement of Published Work Contained in The Thesis}

The publishers of the papers comprising Chapters 2A, 2B, 3B, 4 and 5 hold the copyright for that content, and access to the material should be sought from the respective journals and conference proceedings. The remaining non-published content of the thesis, Chapters $3 \mathrm{~A}$ is submitted and under review, and may be made available for loan and limited copying and communication in accordance with the Copyright Act 1968.

Monaaf Dheaa Abdulradha Al-Falahi

Date: $12 / 07 / 19$ 


\section{Statement of Co-Authorship}

The following people and institutions contributed to the publication of work undertaken as part of this thesis:

\begin{tabular}{|c|c|}
\hline Candidate & $\begin{array}{l}\text { Mr Monaaf Al-Falahi } \\
\text { Australian Maritime College } \\
\text { College of Sciences and Engineering } \\
\text { University of Tasmania }\end{array}$ \\
\hline Author 1 (Primary Supervisor) & $\begin{array}{l}\text { Dr Shantha Gamini Jayasinghe } \\
\text { Australian Maritime College } \\
\text { College of Sciences and Engineering } \\
\text { University of Tasmania }\end{array}$ \\
\hline Author 2 (Secondary Supervisor) & $\begin{array}{l}\text { Dr Hossien Enshaei } \\
\text { Australian Maritime College } \\
\text { College of Sciences and Engineering } \\
\text { University of Tasmania }\end{array}$ \\
\hline Author 3 & $\begin{array}{l}\text { Professor Josep M. Guerrero } \\
\text { Institute of Energy Technology } \\
\text { Aalborg University }\end{array}$ \\
\hline Author 4 & $\begin{array}{l}\text { Professor Udaya Madawala } \\
\text { Department Electrical and Computer Engineering } \\
\text { Faculty of Engineering } \\
\text { University of Auckland }\end{array}$ \\
\hline Author 5 & $\begin{array}{l}\text { Dr Craig Baguley } \\
\text { School of Engineering, Computer and Mathematical } \\
\text { Sciences } \\
\text { Auckland University of Technology }\end{array}$ \\
\hline Author 6 & $\begin{array}{l}\text { Dr Tomasz Tarasiuk } \\
\text { Department of Marine Electrical Power Engineering } \\
\text { Gdynia Maritime University }\end{array}$ \\
\hline Author 7 & $\begin{array}{l}\text { Dr Vikram Garaniya } \\
\text { Australian Maritime College } \\
\text { College of Sciences and Engineering } \\
\text { University of Tasmania }\end{array}$ \\
\hline Author 8 & $\begin{array}{l}\text { Mr Zheming Jin } \\
\text { Institute of Energy Technology } \\
\text { Aalborg University }\end{array}$ \\
\hline Author 9 & $\begin{array}{l}\text { Mr Kutaiba Sabah } \\
\text { Australian Maritime College } \\
\text { College of Sciences and Engineering } \\
\text { University of Tasmania }\end{array}$ \\
\hline Author 10 & $\begin{array}{l}\text { Mr James Coleiro } \\
\text { Australian Maritime College } \\
\text { College of Sciences and Engineering } \\
\text { University of Tasmania }\end{array}$ \\
\hline
\end{tabular}




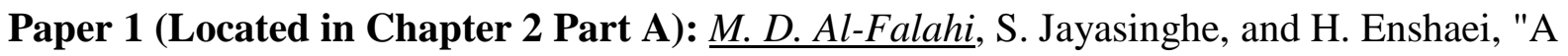
review on recent size optimization methodologies for standalone solar and wind hybrid renewable energy system," Energy Conversion and Management, vol. 143, pp. 252-274, 2017. [Candidate was the primary author and contributed 85\% of the work with Author 1 and Author 2 assisted in review and refine the paper].

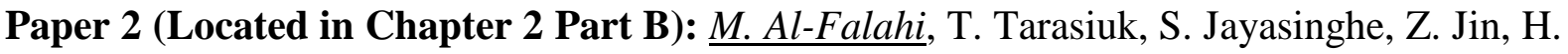
Enshaei, and J. Guerrero, "AC ship microgrids: control and power management optimization," Energies, vol. 11, p. 1458, 2018.

[Candidate was the primary author and contributed 65\% of the work with Author 1 assisted in writing some parts and refining the paper, Author 2, 3 and 8 reviewed the paper and Author 6 contributed in providing experimental data and assisted in control part].

Paper 3 (Located in Chapter 3 Part A): M. D. A. Al-Falahi, S. G. Jayasinghe, H. Enshaei, C. Baguley, and U. Madawala, " Diesel, Hybrid or Fully Electric Ferry, A Method for Operating Cost Analysis and Battery Size Optimization," 2019 9th International Conference on Power and Energy Systems (ICPES). Accepted, 2019.

[Candidate was the primary author and contributed 85\% of the work with Author 1 assisted in reading the refining the paper, Author 2, 4 and 5 assisted in review the paper].

Paper 4 (Located in Chapter 3 Part B): M. D. A. Al-Falahi, J. Coleiro, S. G. Jayasinghe, H. Enshaei, V. Garaniya, C. Baguley, et al., "Techno-Economic Feasibility Study of BatteryPowered Ferries," presented at the Power Electronics Conference (SPEC), IEEE Annual Southern, Singapore, 2018.

[Candidate was the primary author and contributed 75\% of the work, Author 1 assisted in reading the refining the paper, Authors 2,4,5 and 7 assisted in reviewing the paper and author 10 assisted in the economic analysis part]. 
Paper 5 (Located in Chapter 4): M. D. Al-Falahi, K. S. Nimma, S. D. Jayasinghe, H. Enshaei, and J. M. Guerrero, "Power management optimization of hybrid power systems in electric ferries," Energy Conversion and Management, vol. 172, pp. 50-66, 2018.

[Candidate was the primary author and contributed 85\% of the work, Authors 1 and 2 assisted in reading the refining the paper, Author 3 assisted in reviewing the paper and Author 9 assisted in the optimization part].

Paper 6 (Located in Chapter 5): M. D. Al-Falahi, S. Jayasinghe, and H. Enshaei, "Hybrid Algorithm for Optimal Operation of Hybrid Energy Systems in Electric Ferries," Energy, vol.187, p115923.

[Candidate was the primary author and contributed 85\% of the work with Author 1 and Author 2 assisted in reviewing and refining the paper].

We the undersigned agree with the above stated "proportion of work undertaken" for each of the above published (or submitted) peer-reviewed manuscripts contributing to this thesis Signed:

Dr Shantha Gamini Jayasinghe

Primary Supervisor

University of Tasmania

Date: $12 / 07 / 19$ for

Mr Michael van Balen

Head of School

University of Tasmania

Date: 18/07/2019 


\section{Other publications}

During the candidature period, the candidate has contributed in other projects in the field of power management and size optimization of hybrid energy systems in conjunction with the main project presented in this thesis. The methods used in these projects are relevant to the methods applied in this thesis. However, these publications are not included in the body of this thesis as the methods were applied mainly on land-based hybrid power systems. The participation in these projects has helped the candidate to develop the power management strategy used in the main project.

1. M. D. Al-Falahi, K. S. Nimma, S. Jayasinghe, and H. Enshaei, "Sizing and modeling of a standalone hybrid renewable energy system," in Power Electronics Conference (SPEC), IEEE Annual Southern, 2016, pp. 1-6.

2. S. G. Jayasinghe, M. Al-Falahi, H. Enshaei, N. Fernando, and A. Tashakori, "Floating power platforms for mobile cold-ironing," in Power Electronics Conference (SPEC), IEEE Annual Southern, 2016, pp. 1-5.

3. K. S. Nimma, M. D. Al-Falahi, H. D. Nguyen, S. Jayasinghe, T. S. Mahmoud, and M. Negnevitsky, "Grey wolf optimization-based optimum energy-management and battery-sizing method for grid-connected microgrids," Energies, vol. 11, p. 847, 2018 


\section{Acknowledgments}

I would like to thank my supervisors, Dr Shantha Gamini Jayasinghe and Dr Hossien Enshaei, for their continued support and guidance throughout my candidature. The efforts and guidance they provided kept me on track and helped me to face many challenges throughout my candidature.

I would like to thank all staff and colleagues at the University of Tasmania who helped me throughout my research journey.

Finally, I would like to thank my parents, Dr Dheaa and Dr Amal, for their sacrifices and the efforts they have made to provide me the best through my life. Their support and advises throughout this adventure have been essential. Also, I would like to thank my brothers, Dr Omar and Mr Muath, for their support and encouragement. 
I dedicate this thesis to my parents. 


\begin{abstract}
Ferries, which transport passengers, vehicles, and cargo, are encountering new opportunities and challenges in the transportation industry. In particular, fluctuations in the oil price and strict emission regulations imposed by the International Maritime Organization and other governing bodies are the main concerns of the contemporary ferry industry. Therefore, the industry and academia are actively exploring avenues for emission reduction and fuel efficiency improvement.
\end{abstract}

Integration of more-electric technologies (METs), such as electric propulsion, into ferries has been identified as a promising approach to reduce emissions and operating cost. Further developments in the MET approach have decreased the fossil fuel-generated power on board ferries while renewable energy sources (RESs) such as fuel cells and solar cells are added to fill the gap. However, typical power levels and operating characteristic of these RESs do not meet load requirements in ferries and thus, battery energy storage has become an essential element in such hybrid systems to ensure reliable supply of power.

The presence of various sources and different types of loads, such as propulsion and service loads, make the ferry power system a complex network. Effective and efficient management of such a system to achieve the optimal use of available energy is inaccurate with traditional approaches. This gets further complicated with the presence of various constrains and operating requirements of each individual unit. Thus, advanced power management strategies are required to achieve optimal operation of future more-electric ferries.

This study aims to develop, evaluate, and compare the performance of classical and metaheuristic algorithm-based power management systems (PMSs) for hybrid power systems in short-haul electric ferries for fuel efficiency improvements and emission reductions. With the current trends in the industry, emission reduction and fuel efficiency improvement are taken as operational cost functions for the optimization. Moreover, reliability parameters such as safe operating region, available energy, and battery lifetime are taken as constraints in the optimization process. The developed PMSs should minimize the operational cost function and optimally manage the uncertainties of the short-haul ferry power system.

A simulation model of a direct current (DC) ferry power system is developed in MATLAB/Simulink to validate the developed PMSs. A DC distribution system is chosen in this study due to the fact that majority of energy storage technologies are DC systems. 
Moreover, drawbacks of alternating current (AC) distribution system such as harmonics, reactive power, and the need for synchronizations are not present in DC systems. In addition, the elimination of rectifier stage in variable speed drives reduces the power loss and cost. Therefore, future domestic ferry power systems will use DC distribution over AC. The developed model is simulated for different scenarios and validated with actual data measured from a short-haul domestic diesel-electric driven ferry.

In terms of battery sizing, the main parameters that can affect the battery size for shorthaul ferries are battery depth-of-discharge (DoD) and ferry operational profile (number of stops at terminal, number of cruising periods, and maximum load). The influence of the DoD is more significant than other parameters on the size and lifetime of the battery. This condition also affects the payback period (PBP) of the system. Large DoD value results in low battery size capacity, small PBP, and short battery lifetime. These factors are taken and embedded into the equations to get more realistic results from the optimization process.

Results of the study show that, in terms of PMSs, single meta-heuristic optimization algorithm provides better fuel consumption and emission reductions compared to classical method that uses pre-determined conditions. However, hybrid meta-heuristic optimization algorithm provides further promising optimization results due to its high capability to converge to feasible solutions under different conditions, unlike single meta-heuristic optimization algorithms.

In summary, this study suggests that the hybrid power system using DC distribution with battery storage and hybrid meta-heuristic-based PMS is an efficient combination to achieve low fuel consumptions, lower emissions, and reduced in-port noises for short-haul electric ferries. The insight gained from this study can assist marine coastal vehicles operating in close proximity to residential areas having high concern on gas pollutant emissions. This study also enables other researchers and designers in the field of marine power systems to adapt and extend the methods to other marine vessels. 


\section{Table of Contents}

Declarations ...............................................................................................................................................ii

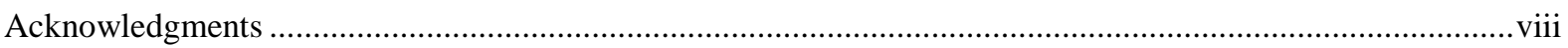

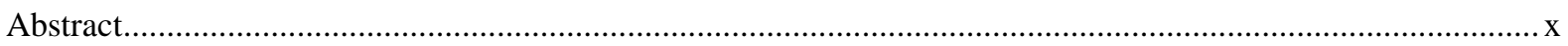

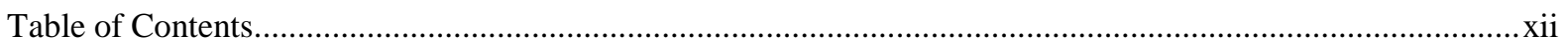

List of Figures....................................................................................................................................

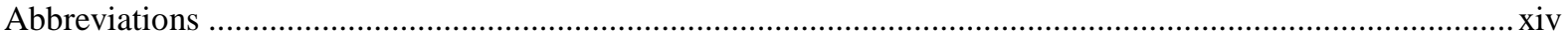

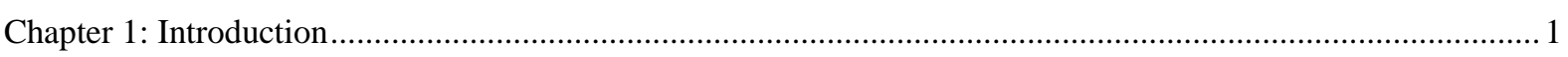

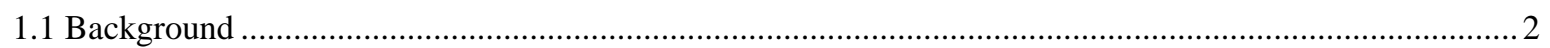

1.1.1 Shipboard power systems....................................................................................................

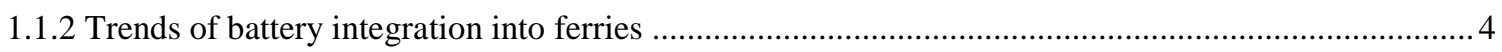

1.1.3 Optimization techniques for hybrid power systems ...................................................................... 7

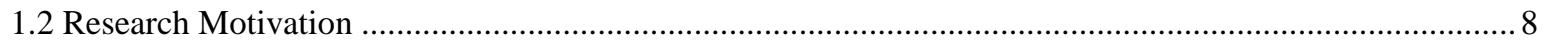

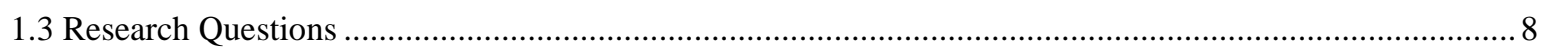

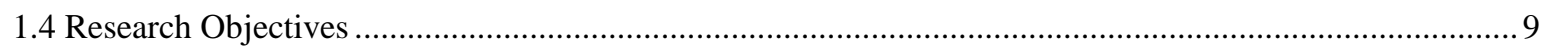

1.5 Research Methodology ............................................................................................................. 9

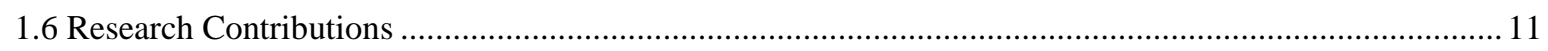

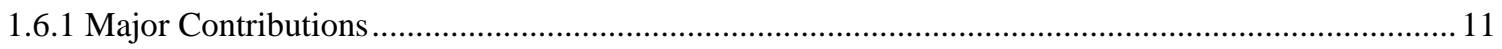

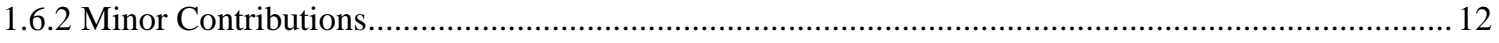

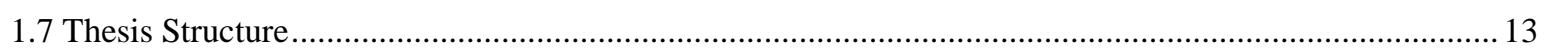

Chapter 2: Optimization Methods and Shipboard Power Systems Configurations ...............................................17

2.1 Part A: Optimization Methods in Hybrid Power Systems ....................................................................... 18

2.2 Part B: Shipboard Power Systems: Configurations and Power Management .............................................. 42

Chapter 3: Comparison of Several Operational Scenarios of Electric Ferries ....................................................... 64

3.1 Part A: Comparison Between Hybrid and Fully Electric Ferries Based on Operational Cost and Battery

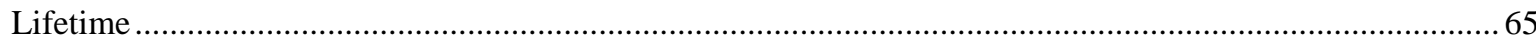

3.2 Part B: Techno-Economic Feasibility Study of Battery- Powered Ferries ................................................ 75

Chapter 4: Application of Classical and Meta-Heuristic-Based Optimization for Electric Ferries ........................ 84

Chapter 5: Performance Improvements of Using Hybrid Meta-Heuristic-Based Optimization Compared to

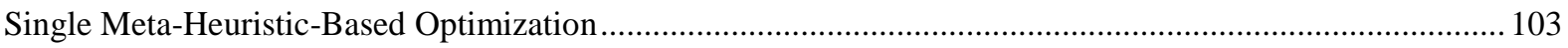

Chapter 6: Summary, Findings, Conclusions and Future Works ............................................................ 120

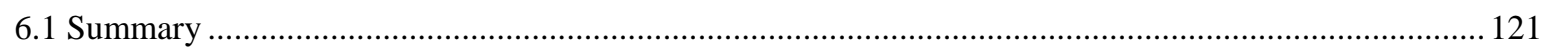

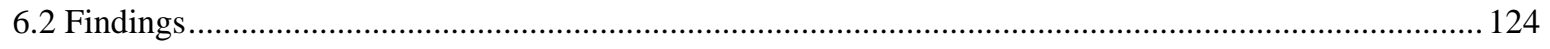

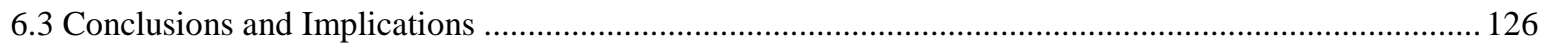

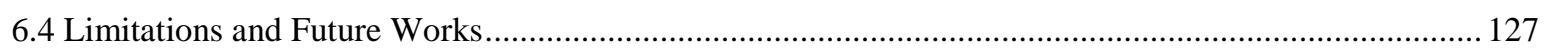

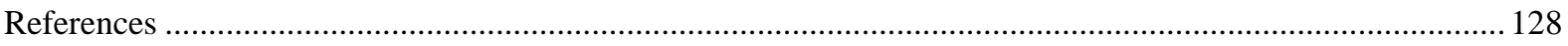




\section{List of Figures}

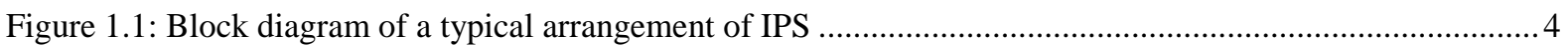

Figure 1.2: Number of hybrid and battery-powered ferries from 2013 to 2018 (Data collected from [14-26]) ..... 5

Figure 1.3: Summary of hybrid and battery-powered ferries in operation from 2013 to 2018 (Data collected from

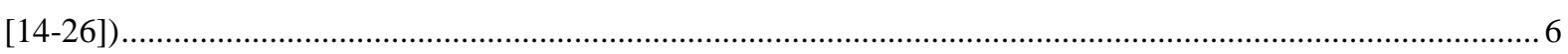

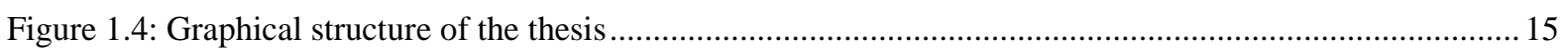




\section{Abbreviations}

AC

Alternating Current

DC

Direct Current

DoD

Depth-of Discharge of Battery

ECA

Emission Control Area

EEDI

Energy Efficient Index

ESS

Energy Storage System

FL-GWO

Fuzzy Logic-Grey Wolf Optimization

GWO

Grey Wolf Optimization

HV

High Voltage

IMO

International Maritime Organization

IPS

Integrated Power System

LV

Low Voltage

LNG

Liquefied Natural Gas

MARPOL

International Convention for The Prevention of Pollution from Ships

MET

More-Electric Technologies

$\mathrm{NO}_{\mathrm{x}}$

Nitrogen Oxide

PBP

Payback Period

PMS

Power Management System

RES

Renewable Energy Source

SFOC

Specific Fuel Oil Consumption

$\mathrm{SO}_{\mathrm{x}}$

Sulphur Oxides

SoC

State-of-Charge of Battery

SPS

Shipboard Power System 



\section{Chapter 1: Introduction}

This chapter introduces shipboard power systems, trends in ferry electrification, and optimization methods. Moreover, it presents the motivation for this research, research questions, objectives, novelty and thesis structure. The thesis incorporates publications, where all chapters are based on peer-reviewed journal and conference papers. A summary of each paper and its contribution to the narrative as a whole is provided in this chapter. A brief introduction to each paper is provided at the start of each chapter. 


\subsection{Background}

Electric propulsion was first introduced in the late 1830s by Moritz Hermann, a German scientist, using a simple $1 \mathrm{~kW}$ direct current (DC) motor to run a small boat [1]. Thereafter, the technology progressed slowly, and the widespread use of electric propulsion in commercial applications started only after 1980, in line with the rapid development of power electronic converter technologies. These converter technologies enable variable speed operation and thus improve the fuel efficiency. In addition, enhanced control flexibility, improved maneuvering capability, fast dynamic response, low noise and vibration, flexibility in engine placement, and low maintenance are other immediate advantages of electric propulsion. Nevertheless, the reduced emission, which is a result of low fuel consumption, is becoming a prominent reason for choosing electric propulsion at present, especially for large cruise ships [2]. International convention for the prevention of pollution from ships (MARPOL) imposed regulations on marine vessels to reduce emissions of nitrogen oxide $\left(\mathrm{NO}_{\mathrm{x}}\right)$ and sulfur oxides $\left(\mathrm{SO}_{\mathrm{x}}\right)$ and stated requirements of a minimum efficiency level [3]. Moreover, the new emission regulations imposed by the International Maritime Organization (IMO) along with the growing global environmental concerns are causing a major shift in the approach of the industry to propulsion system design and have increased the demand for environmentally friendly marine power system solutions [4]. In addition, fluctuations in oil prices require incentives to investigate more technologically advanced and efficient solutions than before to reduce the operational expenses.

Despite the advantages of electric propulsion, prime mover-driven generators remain the source of electrical power. Therefore, the industry and academia are actively exploring various technologies that help reduce emissions and save energy ranging from the use of low emission fuels such as liquefied natural gas to more electrification through increasing the hybridization [5]. In the same context, IMO defined the energy efficiency design index (EEDI) to control and limit emissions of vessels [6]. All these requirements motivate industry and academia to investigate new technologies for reducing emission and fuel consumption in the maritime transportation industry.

\subsubsection{Shipboard power systems}

Owing to the progressive development in power electronic devices, the integration of moreelectric technologies (METs) is gaining increasing attention in the marine industry. As a result, the integration of renewable energy sources (RESs) and energy storage systems (ESSs) is 
becoming popular in the maritime industry due to the growing demand for emission reductions and fuel efficiency improvements.

Shipboard power systems generally follow the shore practice. Thus, $400 \mathrm{~V} / 50 \mathrm{~Hz}$ or 440 V/60 Hz three-phase low-voltage (LV) alternating current (AC) distribution systems are common in most of the ships. This implementation allows normal industrial equipment, which is designed to withstand harshness at sea, to be used on ships. This equipment generally constitutes the auxiliary service loads of ships. Propulsion loads are considerably higher than the above-mentioned service loads. Thus, the LV distribution system is insufficient to cater to these loads. Therefore, high-voltage (HV) three-phase distribution systems, such as 3.3, 6.6, and $11 \mathrm{kV}$, are used to transmit power to these loads. The introduction of power electronic devices has enabled powering of propulsion motors and service loads through the common bus [7]. This arrangement is known as integrated power system (IPS) in which all energy sources, propulsion motors, and service load are connected to the same network. This setting allows high component arrangement flexibility, increased overall system efficiency, and reduced number of prime movers. Therefore, the trend has now been shifted from segregated power systems to IPSs [8].

A typical arrangement of IPS is shown in Figure $1.1[1,4,9]$. The system is composed of several components connected to the same distribution network. Generation units consist of the prime movers coupled with alternators. The power converters are used to convert AC to DC voltages, and vice versa. The power convertors are connected to the generation units if the type of distribution network is DC. Power convertors also needed for propulsion and service loads depend on the types of distribution network, propulsion motor, and service loads. RESs and ESSs are optional. They are used in new ship IPS to reduce dependency on prime movers for generating power and reducing fuel consumption and emission [4]. 


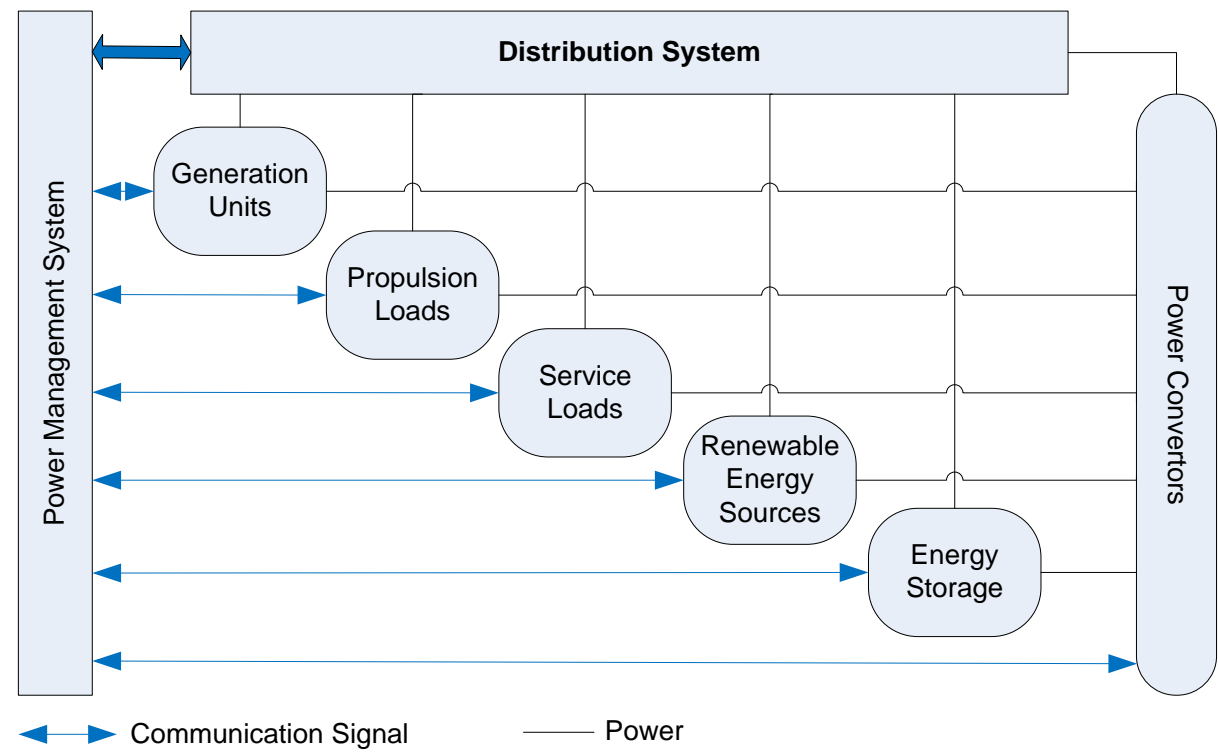

Figure 1.1: Block diagram of a typical arrangement of IPS

Although the overwhelming majority of present electric vessels use IPS with AC distribution systems, the penetration of power electronic converters into power systems is increasing the trend toward DC distribution systems [10]. Compared with a shipboard AC system, DC system provides more flexibility as prime movers and can be controlled at its optimal speeds and maximum efficiency. This attribute to reduce the fuel consumption and emission and increases fuel saving [4]. It also offers additional advantages of space and weight savings, flexible arrangement of equipment, and noise reduction of a diesel engine in the harbor [1]. Moreover, DC shipboard power system enables easy integration of RESs and ESS [6].

The above-mentioned advantages of DC distribution systems have increased the integration of RESs and ESSs into ship power systems. However, typical power levels and densities of RESs remain inadequate to meet the requirements of large ships. In addition, large ESSs are required to satisfy their loads; thus, technical and economic factors need to be considered to justify the hybridization for large ships with RESs or EESs or both [11].

\subsubsection{Trends of battery integration into ferries}

Short-haul ferries, which operate for a short distance with a relatively low power demand, are suitable for the integration of RESs and EESs [12]. Thus, all-electric and hybrid-electric ferries are practically achievable, and the integration of RESs greatly reduces their emissions. However, the slow dynamics or intermittent nature of RESs prevents them from being the main source of power in ferries. Thus, battery ESS has become an integral part in such systems to ensure reliable supply of power. Accordingly, the trend toward integration of batteries into 
ferries has gained close attention in recent years $[12,13]$. This trend can be observed clearly in Figure 1.2.

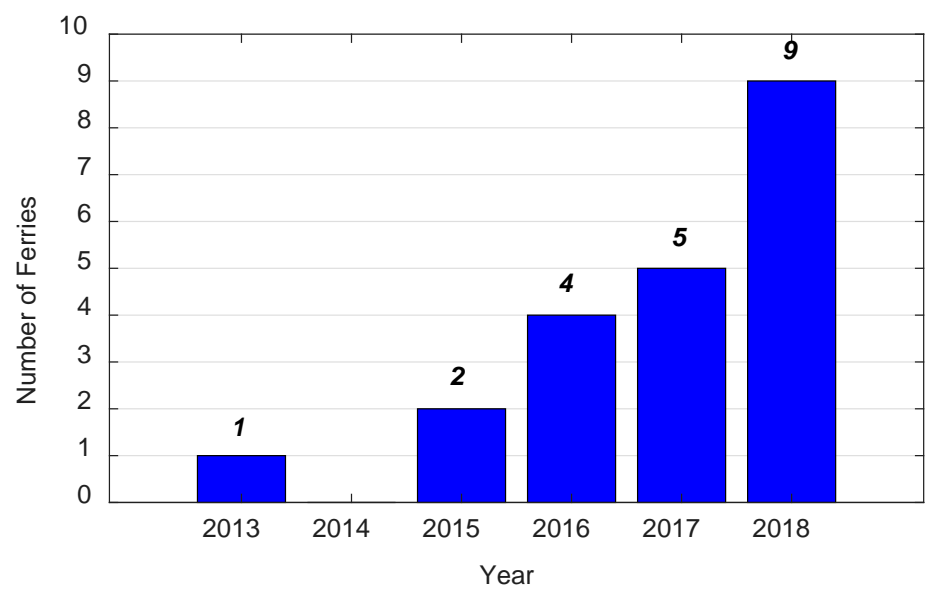

Figure 1.2: Number of hybrid and battery-powered ferries from 2013 to 2018 (Data collected from [14-26])

MV Halaing, the first hybrid electric ferry with battery storage, started its operation in 2013 and recorded significant fuel savings and emission reductions [14]. Following the same trend, Ampere ferry in Norway, which is the first battery-powered ferry in the world, started its operation in 2015 and reported 60\% savings of fuel in 2016 and zero emissions [14]. Another successful example is Finland's first hybrid ferry, Elektra, which started operating in 2017 using batteries as main power supply with diesel generators, reduced $60 \%$ of $\mathrm{CO}_{2}$ emissions with low electricity costs per crossing, and provided large operating cost savings [16]. These successful implementations have attracted many ferry operators and owners to invest in hybrid and battery-powered ferries especially in Europe due the introduction of emissions control areas (ECAs) in the European waters [12]. The investment in such clean ferries is not only on building new ferries with battery storage systems but also on retrofitting some of current electric ferries by integrating a battery system. Such battery integration into existing dieselelectric ferries can provide up to 65\% of emission reduction with high operating cost savings. This scheme was founded by a major project co-financed by European Union in which several diesel-electric ferries was retrofitted to hybrid battery-diesel electric ferries, such as $\mathrm{M} / \mathrm{F}$ Yycho Brahe in Demark [15]. Figure 1.3 shows a summary of the current battery-powered and hybrid ferries in operation since the first hybrid electric ferry with battery storage was introduced in 2013 to the end of 2018. 

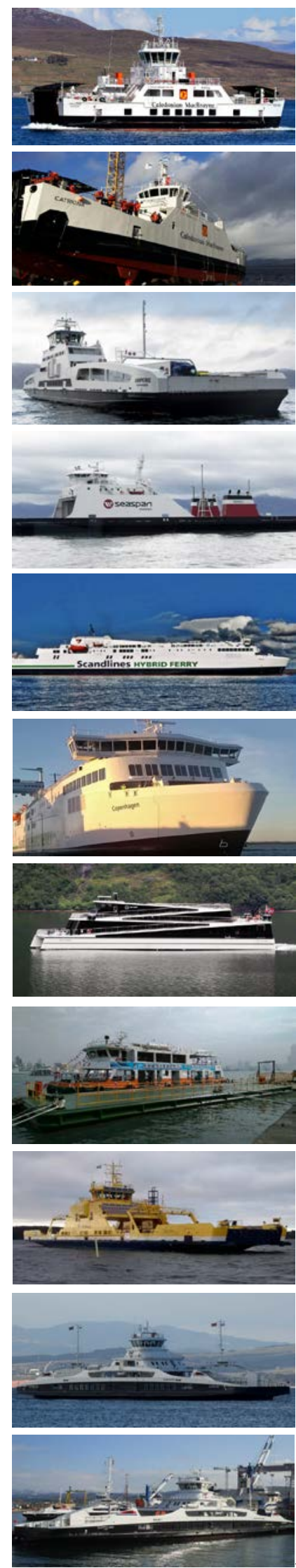

Figure 1.3: Summary of hybrid and battery-powered ferries in operation from 2013 to 2018 (Data collected from [14-26])

MV Hallaig, UK Year: 2013

Owner: Caledonian Maritime Assets Type: Ro-ro ferry

Capacity: 23 cars, 150 passengers Power system: Hybrid Batterydiesel

MV Catriona, UK Year: 2015

Owner: Caledonian Maritime Assets Type: Ro-ro ferry

Capacity: 23 cars, 150 passengers Power system: Hybrid Batterydiesel

Ampere ferry, Norway Year: 2015 Owner: Norland Type: Ro-ro ferry

Capacity: 120 cars, 360 passengers Power system: Battery-powered ferry

Seaspan Swift, Canada Year: 2016

Owner: Seaspan Ferries Type: Ro-ro cargo ferry Capacity: Cargo

Power system: Hybrid Batterydiesel-LNG

M/V Berlin, Germany Year: 2016 wner: Scandlines ype: Ro-ro ferry Capacity: 460 cars, 1300 passengers Power system: Hybrid Battery-

$\mathrm{M} / \mathrm{V}$ Copenhagen, Germany Year: 2016

Owner: Scandlines

Type: Ro-ro ferry

Capacity: 460 cars, 1300 passengers Power system: Hybrid Batterydiesel

Vision of The Fjords, Norway Year: 2016

Owner: The Fjords

Type: passenger ferry Capacity: 400 passengers Power system: Hybrid Battery-

Ferry happiness, Taiwan Year: 2017

ype: Passengers ferry Capacity: 100 passengers Power system: Hybrid Batterydiese

The Elektra, Finland Year: 2017

Owner: FinFerries

Type: Ro-ro ferry Power system: Hybrid Batterydiesel-solar

MF Eidsfjord, Norway Year: 2017

Owner: Fjord1

Type: Ro-ro ferry

Capacity: 120 cars, 349 passenge Power system: Battery-powered

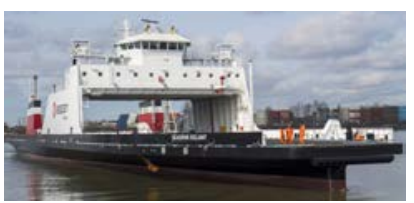

Seaspan Reliant, Canada Year: 2017

Owner: Seaspan Ferries

Corporation

Type: Ro-ro ferry

Capacity: 90 cars

Power system: Hybrid Batterydiesel-LNG

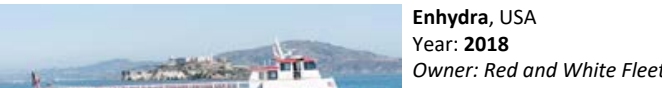

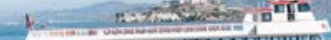

Owner: Red and White Fleet

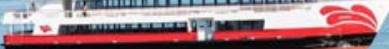

Type: Passenger ferry

Capacity: 600 passenger

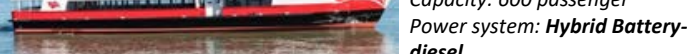
diesel

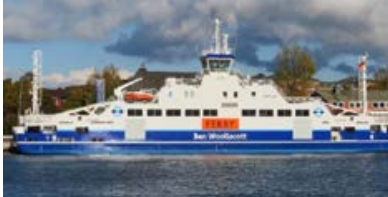

Ben Woollacott, UK

Year: 2018

Owner: The Transport for London Type: Ro-ro ferry

Capacity: 45 cars, 150 passenger Power system: Hybrid Batterydiese

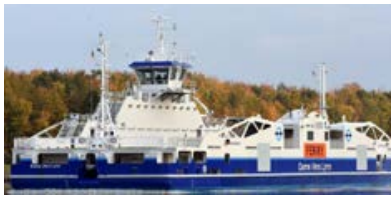

Vame Vera Lynn, UK

Year: 2018

Owner: The Transport for London Type: Ro-ro ferry

Capacity: 45 cars, 150 passenger Power system: Hybrid Batterydiese

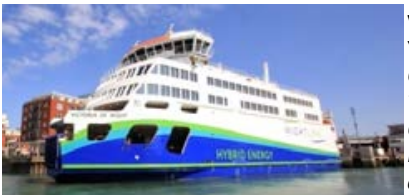

ictoria of Wight, UK Year: 2018 Owner: Wightlink Type: Ro-ro ferry

Capacity: 178 cars, 1175 passenger Power system: Hybrid Batterydiese

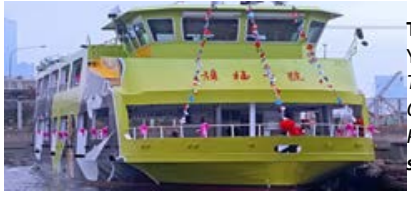

u No.1, Taiwan

Yr: 2018

Type: Passenger ferry

Capacity: 150 passenger, 46 bicycle Power system: Hybrid Battery olar-diese

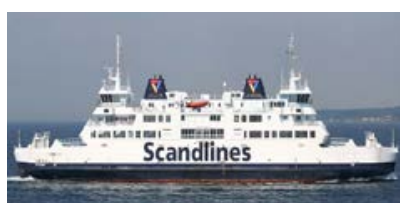

/F Tycho Brahe, Denmark

ear: 2018 (Retrofit with battery) Owner: HH Ferries

Capacity: 240 cars, 1250 passenger diesel

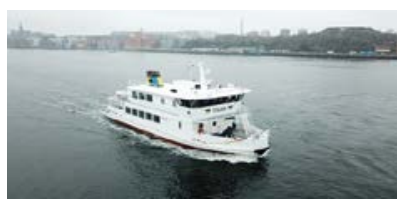

-class, Sweden

Year: 2018

Owner: Waxholmsbolaget

Type: Passenger ferry

Capacity: 150 passenger

Power system: Hybrid Batterydiesel

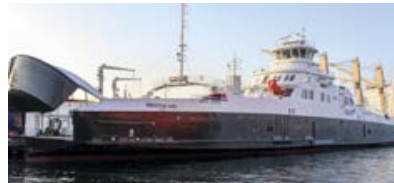

$\emptyset$ kstrafjord, Norway

Year: 2018

Owner: Fjord

Type: Ro-ro ferry

Capacity: 130 cars, 299 passenge Power system: Hybrid Batterydiesel

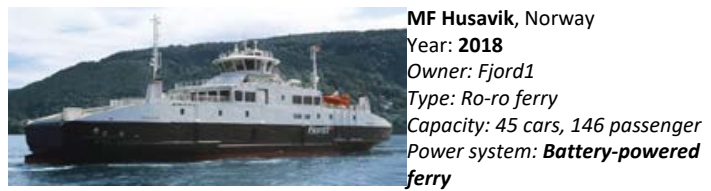

MF Gloppefjord, Norwa

: Ro-ro ferry

Capacity: 120 c

tem: Battery-powered 
This trend continuous as an increasing number of ferries are being built with hybrid and fully battery-powered system due to their advantages of fuel savings and emission and noise reductions, especially as most ferries operate close to human habitation areas where emissions and noises are of concerns [14, 27]. Many battery-powered and hybrid ferries and boats that move through inland waterways in the Netherlands, Norway, Belgium, and Canada are being built and soon to make their first voyage [28-30]. Only Norway will renew and build over 20 ferries with a battery storage system in the next few years [12].

The challenge in such hybrid system is matching the characteristic of the battery storage system (discharge and charge rates) and other generation units to meet the load requirements. The way that the system is operated can significantly affect the operating costs and the battery lifetime. Moreover, the efficient operation of the system will maximize the benefits of using such hybrid- and all-electric systems with batteries. In this context, the development of efficient power management strategies along with the progressive development in batteries will help further increase the trend of battery-powered and hybrid electric ferries with battery storage systems.

\subsubsection{Optimization techniques for hybrid power systems}

The use of optimization techniques is essential to operate the power systems optimally and efficiently. The presence of highly dynamic loads and the integration of multiple energy generation units and storage systems with several interfacing power electronic converters increase the complexity of the electrical power system onboard ferries. This condition requires an advanced optimization technique to optimally match the power generation and load demand while optimizing the use of available resources.

Hybrid power system optimization techniques can be classified into classical and modern techniques. Classical techniques use iterative, numerical, analytical, probabilistic, and graphical construction methods [3]. These methods utilize differential calculus in deriving the optimal solution [20]. Modern techniques use meta-heuristic and evolutionary algorithms $[3,23]$. These methods can determine the global optimal system and have improved convergence and accuracy in finding a set of optimal solutions [3,25].

The multi-scheme classical technique method, which uses a deterministic rule-based method with preplanned states to manage the power flow, is the simplest power management method. This method has been applied for hybrid ferries and boats in [31-36] and is mostly 
used for hybrid ferry with fuel cell system. Despite being a simple method, several innumerable contingencies may be present during the vessel operation. Thus, pre-planned power management methods can result in suboptimal fuel efficiency.

The advanced power management-based meta-heuristic optimization algorithm is a promising approach to match the power generation and load demand while meeting the operational constrains and reducing fuel consumption and emissions [37]. Furthermore, the use of a hybrid meta-heuristic algorithm (by combining two or more algorithms) can provide more promising optimization results for hybrid energy systems than the single meta-heuristic algorithm [37-40]. Nevertheless, a few studies have applied the meta-heuristic algorithm to solve several optimization problems for shipboard power systems, namely, reconfiguration and restoration of the ship power system [41, 42], optimization of the ship power configuration under several operating conditions [43] and minimization of operational cost and emission [44, 45]. However, the implementation of meta-heuristic optimization for short-haul hybrid ferries with a battery storage system is limited. Therefore, a study to fill this research gap is required to develop, investigate, and assess meta-heuristic optimization-based power management system (PMS) for short-haul hybrid ferries with a battery storage system.

\subsection{Research Motivation}

The trend towards further electrification of ferries is limited by the operational profiles of vessels and the optimization of the system. The introduction of multi-generation sources, storage systems, and interfacing power electronic converters in hybrid- and all-electric ferries increase the complexity of the ferry power system. Effective and efficient management of such a system to achieve optimal use of available energy is ineffective with traditional approaches. Therefore, advanced optimization strategies to maximize fuel savings and minimize operating costs while satisfying all operational constraints can help attract the attention of ferry owners and operators to invest in hybrid- and all-electric ferries.

\subsection{Research Questions}

1. What types of optimization algorithms can improve the design and operation of hybrid power systems while fulfilling the reliability, economic, and environmental criteria?

2. What is the most feasible shipboard power system configuration in terms of reliability, ease of operation, and fuel efficiency? 
3. What are the main parameters in sizing a battery storage system for electric ferries and how they affect the battery life?

4. How a power management strategy can optimize the operation of short-haul electric ferries in terms of fuel consumption, emission reduction, and cost effectiveness?

\subsection{Research Objectives}

1. To investigate the most recent optimization algorithms for hybrid power systems and the most suitable power system configuration for marine electric vessels

2. To identify the main design parameters that affect the lifetime of batteries in the power system of short-haul electric ferries

3. To develop an innovative power management strategy for optimally managing the power sharing of short-haul electric ferries in terms of fuel consumption and emission reduction

4. To compare and evaluate the performance of classical, single meta-heuristic, and hybrid meta-heuristic-based power management strategies for short-haul electric ferries

5. To validate the developed power management strategy by using a set of data captured from a short-haul ferry, which is currently in operation as a case study

\subsection{Research Methodology}

The following seven steps were undertaken to answer the research questions and to fulfill the research objectives. The first two steps were undertaken as comprehensive literature study which looked at different optimization methods and shipboard power system configurations to identify the performance of recent optimization methods and the most suitable power system configurations in hybrid power systems. From step 3, the methods were carried out to achieve the main research objectives.

1. To identify parameters and study the performance of recent optimization algorithms used to optimize standalone hybrid power systems in terms of system reliability, economic advantage, and environmental factors. Research on modern optimization methods and their performance in hybrid marine power systems is limited. Hybrid marine power systems have a lot in common with terrestrial hybrid power systems. Thus, many of the methods and considerable equipment and components at the generation side are the same for them. For this end, a comprehensive literature study was conducted that looked at different optimization methods and their performance for 
terrestrial hybrid power systems (Chapter 2 Part A). The optimization methods for hybrid power systems can be categorized into three categories: classical methods, modern methods, and computer tools. Modern techniques, based on single artificial intelligence algorithms, are becoming more popular than classical algorithms owing to their capabilities in solving complex optimization problems. In addition, hybrid algorithms are preferred over single algorithms mainly due to their ability to provide promising optimization results. Therefore, three methods, classical, single metaheuristic, and hybrid meta-heuristic algorithms, are investigated.

2. Study the shipboard power system configurations and identify the most suitable configuration to achieve optimal operation of the system in terms of fuel consumption and emission reduction (Chapter 2 Part B). The trend toward the use of DC distribution systems onboard is becoming popular. The reason is that DC distribution systems provide an efficient distribution of electric energy by linking AC and DC energy sources through power-electronic devices that customize energy flow to the load. Therefore, DC distribution system is used in this study.

3. Collect and measure data from a short-haul domestic electric ferry to validate the proposed system. Case study and data are analyzed and presented in Chapters 3, 4, and 5.

4. Develop simulation model with PMS based on classical method namely, rule-based method, for electric ferries in MATLAB/Simulink (Chapter 3 Part A) and discuss the parameters that affect the sizing of the onboard batteries (Chapter 3 Part A and B). Three scenarios are simulated: (1) standalone hybrid system, (2) grid-connected hybrid system, and (3) grid-connected fully electric system. Main battery sizing parameters are identified, and optimal sizing of battery storage system is carried out. The operating cost factor is used to assess the performance of each scenario. In addition, a technoeconomic feasibility study is performed to assess the feasibility of battery-powered ferries (Chapter 3 Part B). The technical study includes sizing of the battery storage system based on battery DoD and maximum load scenario. The economic study includes initial investment, operational cost, and maintenance cost of a battery-powered electric ferry. The economic analysis considered the payback period (PBP) and battery lifecycle as assessment factors. 
5. Design, application and comparison of classical rule-based method and single metaheuristic grey wolf optimization (GWO) method to optimally manage the power generation in hybrid ferries (Chapter 4). Moreover, the performance of the DC hybrid power system is compared with that of the conventional AC system. Fuel consumption and emission reduction are used as assessment factors to identify the performance of each method. The specific fuel oil consumption (SFOC) is used in the fuel consumption equation, and the main affecting optimization parameters of the SFOC of onboard engines are identified.

6. Develop a hybrid meta-heuristic algorithm-based PMS for the fuel savings of hybrid electric ferries and compare it with single meta-heuristic algorithm in terms of performance (Chapter 5). The proposed hybrid PMS method applies an interactive approach on the basis of a GWO and fuzzy expert system to improve the computational efficiency of the algorithm. The main objective function of the optimization problem is to reduce the operating cost by minimizing fuel consumption. A general SFOC equation is derived using regression estimation based on fuel consumption data obtained from different engine manufacturers. This equation can be used to estimate fuel consumption for different diesel engines with the same rated power. The optimization problem is decomposed in two subproblems: (1) finding the best battery charging power based on battery SoC and power difference between generation and load and (2) determining the optimal power management for generators and battery. Performance of the proposed fuzzy logic-GWO (FL-GWO) algorithm is compared with that of GWO algorithm and assessed in terms of fuel consumption, operating cost, and standard deviation.

\subsection{Research Contributions}

The novelty of this study is derived from the development of new methods that address the important issues in the field of marine power system. The contribution of the work is divided into major and minor contributions.

\subsubsection{Major Contributions}

1. Develop a novel power management strategy for short-haul hybrid electric ferries:

This study provides a novel hybrid meta-heuristic-based PMS for operating cost and fuel consumption reductions in short-haul hybrid electric ferries. This study is the first to develop a hybrid meta-heuristic algorithm for efficiently managing the 
power system in short-haul hybrid electric ferries with battery storage systems. This study enables other researchers to adapt and extend the proposed methods to other marine vessels.

\section{Test the performance of optimization methods for short-haul electric ferries:}

This study assesses the performance of classical, single meta-heuristic, and hybrid meta-heuristic methods used to optimize the power of short-haul electric ferries with batteries in terms of fuel consumption and emission reduction. This study is the first in the field of marine power systems to address and compare the performance of three optimization approaches in optimizing the power in shorthaul electric ferries with battery storage systems.

\subsubsection{Minor Contributions}

1. Compare different ferry power system configurations in terms of fuel consumption reductions:

This study examines and compares different possible power system configurations for short-haul electric ferries. These configurations are AC power, standalone DC hybrid power, grid-connected DC hybrid power, and grid-connected all-electric systems. The best system configuration in terms of fuel consumptions and operating costs is determined to help researchers and designers in the field of marine power systems test conformance of other configurations.

2. Provide a techno-economic study of battery-powered ferry:

This study is conducted to investigate and justify the applicability of implementing all-electric battery-powered systems for short-haul ferries from economical and technical aspects.

3. Identify the main parameters for battery sizing in short-haul hybrid electric ferries:

Critical parameters which affect the size and lifetime of onboard batteries are identified. This study enables designers to adapt the sizing approach for optimally sizing the onboard batteries for short-haul hybrid electric ferries. This study also enables other researchers to adapt and extend the sizing method to other situations.

4. Propose a simplified approach to estimate the fuel consumption of onboard diesel engines: 
The study presents a simplified approach based on regression estimation to derive SFOC equation for onboard engines. This simple approach can be used to estimate the fuel consumption of engines when detailed information of the engines is unavailable.

\subsection{Thesis Structure}

The thesis incorporates publications of journal and conference papers. Details of the corresponding publications are given at the beginning of each chapter. The thesis consists of six chapters. A graphical structure of the thesis is shown in Figure 1.4. The structure of the thesis is outlined below.

Chapter 1: This chapter provides an introduction and relevant background leading to the research questions and objectives. A summary of the research methodology is also presented, and the novel aspects of this research are outlined.

Chapter 2 (Papers 1 and 2): This chapter is divided into two parts. Part A (Paper 1) provides a comprehensive review and analysis of recent optimization algorithms in the field of hybrid power systems. Performance of several types of optimization method is compared and discussed. Part B (Paper 2) provides a review on shipboard power system architectures. In addition, control techniques and power management strategies that are used or proposed for ship microgrids are discussed.

Chapter 3 (Papers 3 and 4): This chapter is divided into two parts. Part A (Paper 3) provides design and simulation of a classical optimization method, namely, rule-based method, to optimally manage the power system of short-haul ferries for minimizing operating cost. Moreover, an approach to find the optimal battery capacity is presented. The proposed methods are applied for three power system configurations, each of which is assessed in terms of the operating cost factor. Part B (Paper 4) provides a techno-economic analysis of battery-powered ferries. The sizing of the battery system is carried out on the basis of battery DoD and maximum load scenario. In addition, initial investment, operational cost and maintenance cost of a battery-powered electric ferry are discussed and assessed in terms of PBP and battery lifecycle.

Chapter 4 (Paper 5): In this chapter, a meta-heuristic optimization algorithm namely GWO is designed and applied to optimally manage the operation of short-haul hybrid ferries in terms of fuel and emission reduction. The performance of the algorithm is compared with that of classical rule-based method. The simulation results obtained from the existing ferry power 
system are used as a benchmark to show the fuel saving improvements provided by using GWO and rule-based methods.

Chapter 5 (Paper 6): In this chapter, a novel hybrid meta-heuristic algorithm namely fuzzy logic-GWO (FL-GWO) is developed and applied to optimally manage the operation of shorthaul hybrid ferries in terms of operating cost minimization. The performance of FL-GWO is compared and assessed in terms of fuel consumption, operating cost, and standard deviation. In addition, a simplified approach to estimate the fuel consumption of onboard diesel engines is proposed.

Chapter 6: This chapter provides a summary of the thesis, answers to research questions, and concludes the main findings and outcomes of the thesis. In addition, implications of the findings, recommendations, and future work are presented. 


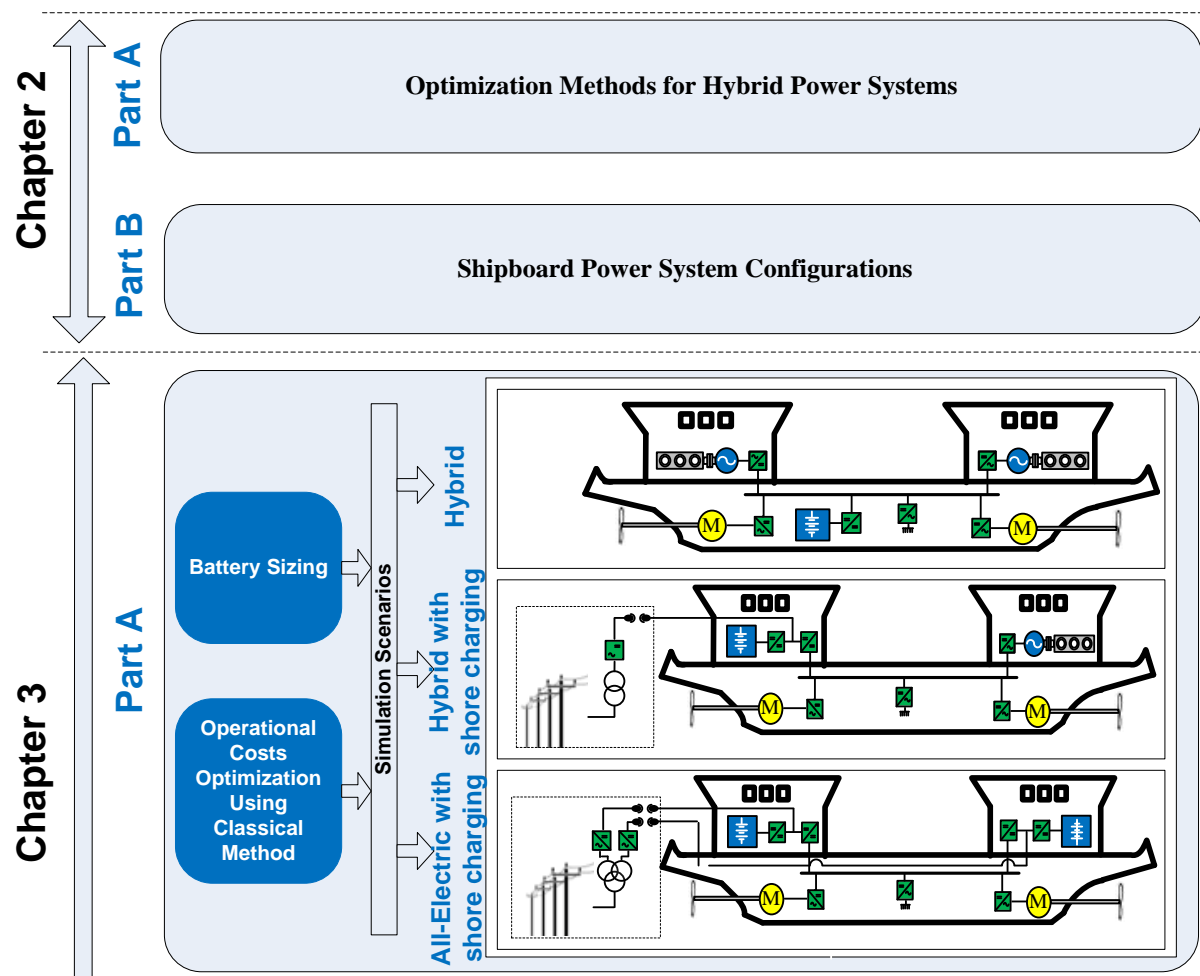

Paper 1: M. D. Äl-Falahi, S Jayasinghe, and H. Enshaei, "A review on recent size optimization

methodologies for standalone solar and wind hybrid renewable energy system," Energy Conversion and Management, vol. 143, pp. 252-274, 2017.

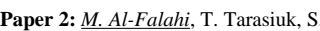
Jayasinghe, Z. Jin, H. Enshaei, and J. Guerrero, "AC ship microgrids: control and power management optimization," Energies, vol. 11, p. 1458, 2018.

Paper 3: M. D. A. Al-Falahi, S. G ayasinghe, H. Enshaei, C. Baguley, and U. Madawala, "Diesel, Hybrid or Fully Electric Ferry, A Method For Operating Cost Analysis And Battery Size Optimization," 2019 9th International Conference on Power Accepted, 2019.

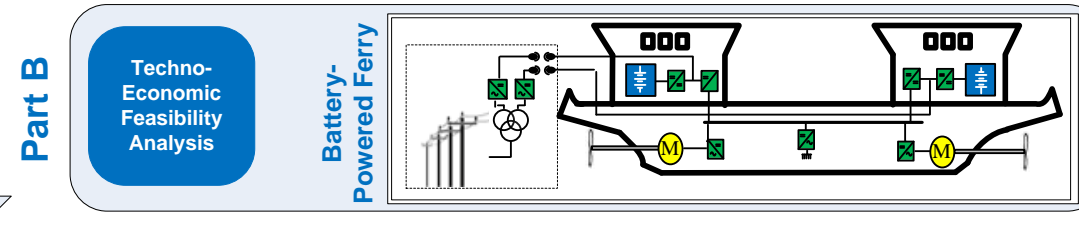

Paper 4: M. D. A. Al-Falahi, J. Coleiro, S. G. Jayasinghe, H. Enshaei, V. Garaniya, C. Baguley, et al., "Techno-Economic Feasibility Study of Battery- Powered Ferries," presented at the Power Electronic Conference (SPEC), IEEE Annual Southern, Singapore, 2018.

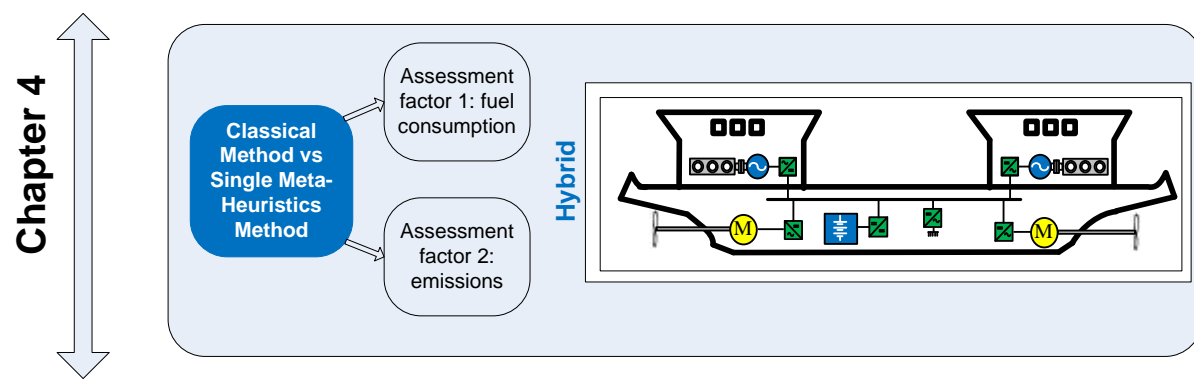

Paper 5: M. D. Al-Falahi, K. S. Nimma, S. D. Jayasinghe, $\mathrm{H}$. Enshaei, and J. M. Guerrero, "Power manageme. M. Guerrero, "Power power syst optimization of hybrid Energy Conversion and Management, vol. 172, pp. 50-66, 2018.

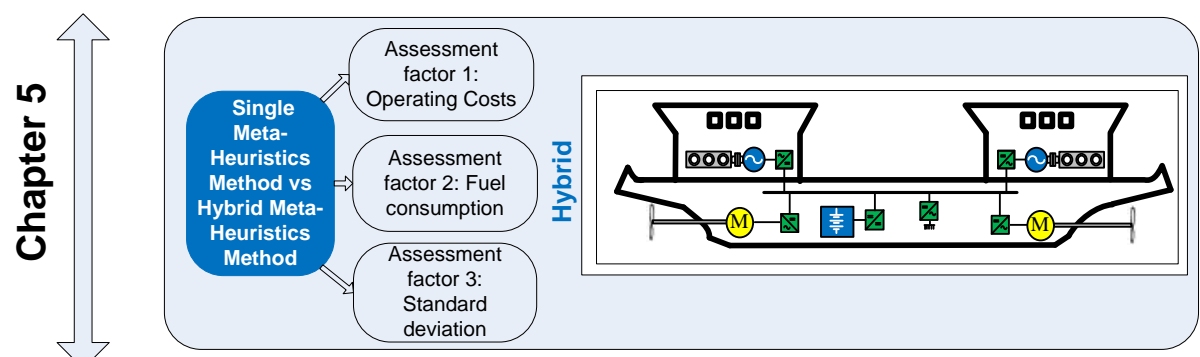

Paper 6: M. D. Al-Falahi, S ayasinghe, and H. Enshaei, "Hybri Algorithm for Optimal Operation of Hybrid Power Systems in Electric Ferries”. Energy, vol.187, p115923.

ט)

Summary, Findings, Conclusions and Future Works

Figure 1.4: Graphical structure of the thesis 


\section{Chapter 2: \\ Optimization Methods and Shipboard Power Systems Configurations}

This chapter provides a comprehensive review and discussion of recent optimization algorithms in hybrid power systems and configurations of shipboard power systems. This chapter consists of two subchapters.

Part A: Optimization Method in Hybrid Power Systems

Part B: Shipboard Power Systems: Configurations and Power Management 


\section{Chapter 2}

\subsection{Part A: Optimization Methods in Hybrid Power Systems}

This part provides a comprehensive review on recent optimization algorithms used to optimize standalone hybrid power systems in terms of reliability, economic, environmental, and social factors. Research on artificial optimizations and their performance in hybrid marine power systems is limited. Given that hybrid marine power systems have same properties as terrestrial hybrid power systems, many of the methods and considerable equipment and components at the generation side are same for them. Thus, a comprehensive literature study is conducted to analyze different optimization methods and their performance for terrestrial hybrid power systems. This subchapter has been published in the Journal of Energy Conversion and Management. The citation of the article is:

Al-Falahi, M. D., Jayasinghe, S. D. G., \& Enshaei, H. (2017). A review on recent size optimization methodologies for standalone solar and wind hybrid renewable energy system. Energy Conversion and Management, 143, 252-274. 
Review

\title{
A review on recent size optimization methodologies for standalone solar and wind hybrid renewable energy system
}

\author{
Monaaf D.A. Al-falahi* ${ }^{*}$ S.D.G. Jayasinghe, H. Enshaei \\ Australian Maritime College, University of Tasmania, Tasmania 7248, Australia
}

\section{A R T I C L E I N F O}

\section{Article history:}

Received 20 January 2017

Received in revised form 13 March 2017

Accepted 5 April 2017

Available online 13 April 2017

\section{Keywords:}

Hybrid energy system

Off-grid

Optimization

Renewable energy sources

Solar

Standalone

Wind

\begin{abstract}
A B S T R A C T
Electricity demand in remote and island areas are generally supplied by diesel or other fossil fuel based generation systems. Nevertheless, due to the increasing cost and harmful emissions of fossil fuels there is a growing trend to use standalone hybrid renewable energy systems (HRESs). Due to the complementary characteristics, matured technologies and availability in most areas, hybrid systems with solar and wind energy have become the popular choice in such applications. However, the intermittency and high net present cost are the challenges associated with solar and wind energy systems. In this context, optimal sizing is a key factor to attain a reliable supply at a low cost through these standalone systems. Therefore, there has been a growing interest to develop algorithms for size optimization in standalone HRESs. The optimal sizing methodologies reported so far can be broadly categorized as classical algorithms, modern techniques and software tools. Modern techniques, based on single artificial intelligence (AI) algorithms, are becoming more popular than classical algorithms owing to their capabilities in solving complex optimization problems. Moreover, in recent years, there has been a clear trend to use hybrid algorithms over single algorithms mainly due to their ability to provide more promising optimization results. This paper aims to present a comprehensive review on recent developments in size optimization methodologies, as well as a critical comparison of single algorithms, hybrid algorithms, and software tools used for sizing standalone solar and wind HRES. In addition, an evaluation of all the possible combinations of standalone solar and wind energy systems, including their assessment parameters of economical, reliability, environmental, and social aspects, are also presented.
\end{abstract}

(c) 2017 Elsevier Ltd. All rights reserved.

\section{Contents}

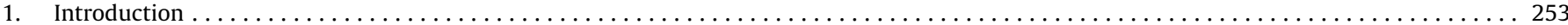

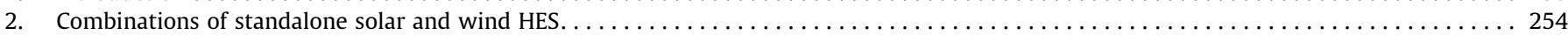

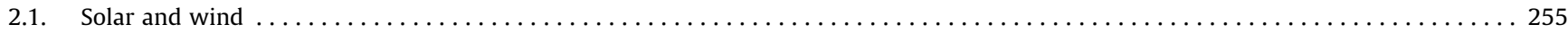

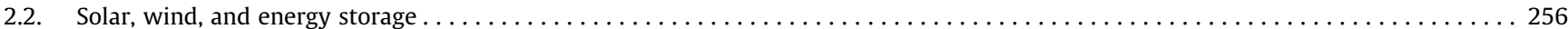

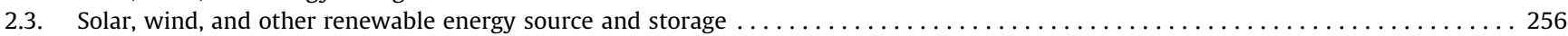

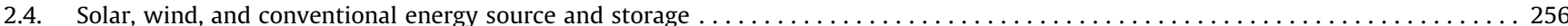

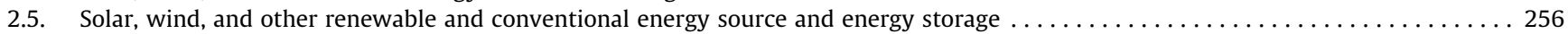

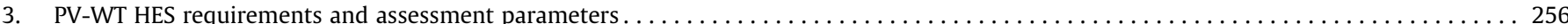

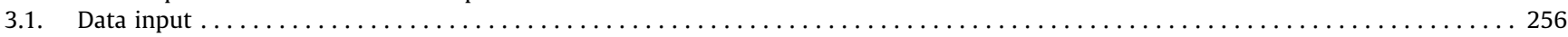

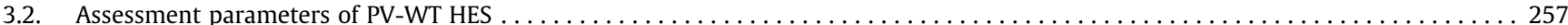

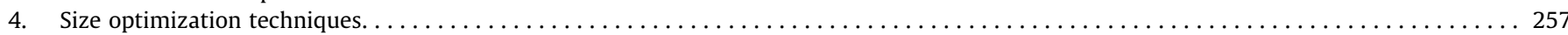

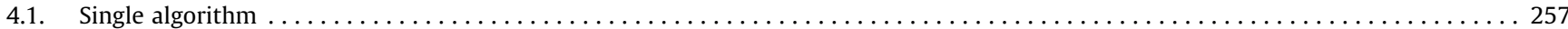

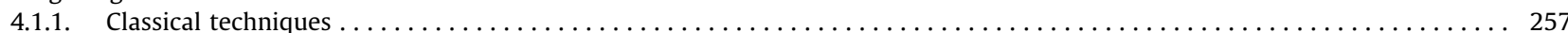

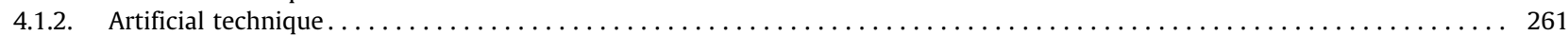

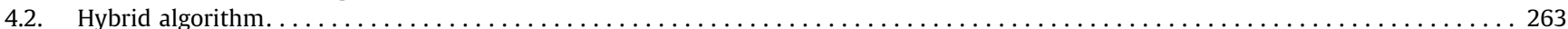

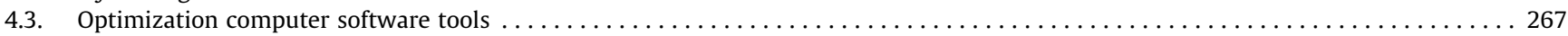

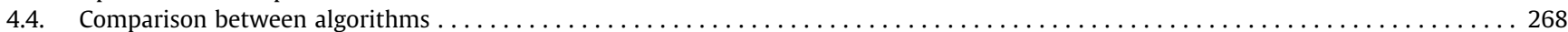

\footnotetext{
* Corresponding author.

E-mail addresses: monaaf.alfalahi@utas.edu.au (M.D.A. Al-falahi), shanthaj@ utas.edu.au (S.D.G. Jayasinghe), hossein.enshaei@utas.edu.au (H. Enshaei).
} 


$\begin{array}{ll}\text { Nomenclature } \\ \text { LPSP } & \text { loss of power supply probability } \\ \text { LOLP } & \text { loss of load probability } \\ \text { LOLR } & \text { loss of load risk } \\ \text { LOLE } & \text { loss of load expectation } \\ \text { LOEE } & \text { loss of energy expectation } \\ \text { UL } & \text { unmet load } \\ \text { DPSP } & \text { deficiency in power supply probability } \\ \text { EENS } & \text { expected energy not supplied } \\ \text { ENS } & \text { energy not supplied } \\ \text { EIR } & \text { energy index of reliability } \\ \text { ELF } & \text { equivalent loss factor } \\ \text { D } & \text { net dump energy } \\ \text { TED } & \text { total energy deficit } \\ \text { WRE } & \text { wasted renewable energy } \\ \text { REP } & \text { renewable energy penetration } \\ \text { FEE } & \text { final excess of energy } \\ K_{1} & \text { energy fluctuation rate } \\ P(R) & \text { risk state probability } \\ \text { ASC } & \text { annual system cost } \\ \text { TIC } & \text { total investment cost }\end{array}$

$\begin{array}{ll}\text { LCC } & \text { life cycle cost } \\ \text { COE } & \text { cost of energy } \\ \text { LCOE } & \text { levelised cost of energy } \\ \text { LEP } & \text { loss of energy probability } \\ \text { TC } & \text { total cost } \\ \text { TAC } & \begin{array}{l}\text { annual total cost } \\ \text { E }\end{array} \\ \text { total } \mathrm{CO}_{2} \text { emissions } \\ \text { EE } & \text { embodied energy } \\ \text { LCA } & \text { life cycle assessment } \\ \text { HDI } & \text { human development index } \\ \text { JC } & \text { job creation } \\ \text { SCC } & \text { social cost of carbon } \\ \text { SOC } & \text { state of charge } \\ \text { TPC } & \text { total precent cost } \\ \text { NPC } & \text { net precent cost } \\ \text { NPV } & \text { net present value } \\ P(H) & \text { percentage of healthy state probability } \\ \text { TC } & \text { total cost }\end{array}$

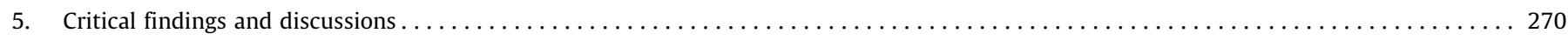

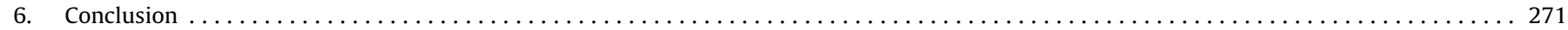

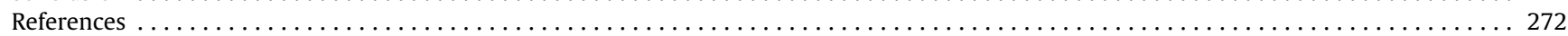

\section{Introduction}

Electrical power is one of the most commonly sought commodities of mankind. Currently, more than $70 \%$ of the global electricity demand is supplied by burning fossil fuels, such as crude oil, coal, and natural gas [1]. With the growth of economies and world population, the demand for electricity increases and as a result the fossil fuel consumption increases. However, conventional fuel reserves are finite and depleting rapidly which require immediate attention and sustainable approaches to avoid potential energy crisis in the future. Additionally, fossil fuels account for harmful emissions, including greenhouse gasses (GHGs), which contribute to the global warming [2,3]. In the current context, these problems are addressed in several ways. One of the popular approach is to widen the public awareness on reducing energy consumption in domestic and industrial spheres and promote energy efficient technologies. Another approach is to promote renewable energy systems (RES) and develop associated technologies to make them reliable, costeffective, environmental friendly and affordable even to the general public to use in their residential applications. The latter has drawn more attention in the research community, industries, and governments and as a result, many countries and regions have taken strong initiatives to increase their renewable energy capacity.

In Europe, the European Technology Platform for Electricity Networks of the Future, also known as ETP Smart Grids (ETP SG) produced the Strategic Research Agenda 2035 (SRA 2035) which expected that by 2020 , approximately $34 \%$ of the total electrical energy consumption will come from renewable energy and will have gone more than that by 2035 [4]. The European Union (EU) council adopted the Energy Roadmap 2050 in June 2012 which declared that decarburization by $80 \%$ reduction (compared with the estimated level in 1990) of GHG emissions in European energy system will be technically and economically feasible. This can be achieved by implementing numerous strategies, such as increasing the development of renewable energy generation, which can be seen clearly where the local and small-scale generation from renewable energy sources has remarkably increased in Europe from $312 \mathrm{GW}$ at the end of 2012 to $380 \mathrm{GW}$ at the end of 2014 [4]. In Italy, $11.4 \mathrm{GW}$ of photovoltaic (PV) power capacity had been connected to the distribution network in December 2012 [4]. In Germany, as of September 2015, RESs accounted for $47 \%$ of installed net generating capacity [5]. Furthermore, the annual energy production at about $38.850 \mathrm{GW}$ comes from PVs in August 2015 keeps Germany with the largest amount of installed PV capacity in the world [5,6], and about $41.353 \mathrm{GW}$ are from onshore and offshore wind turbines in September 2015 [5]. Similar trends are observed in other countries and regions such as the USA $[2,7,8]$, with over $16 \mathrm{GW}$ of installed solar power in 2014 [6]. This trend increased the total installed PV power globally to reach over $177 \mathrm{GW}[4]$.

The aforementioned renewable energy capacities include large scale wind and solar systems, as well as residential PV systems. Majority of the residential PV systems work in the grid connected mode, in which excess power is injected to the grid during the day time and power is received from the grid at night. However, in remote areas where the grid extension is not feasible, HRESs are used in the standalone mode for individual houses or in microgrids (MGs) where several houses are connected to form a small power grid $[9,10]$. The second approach is becoming popular in islands and rural areas [11] as it provides a cost-effective alternative where power grid extensions is expensive and fuel transportation is difficult and costly [12]. Currently, the population in islands is estimated to be over 740 million worldwide based on geographic information system (GIS) analysis [13]. Another study has shown that many islands in the Indian and Pacific Oceans spend up to $30 \%$ of their gross domestic product (GDP) on conventional energy resources, such as fossil fuel [14]. In the Caribbean islands, power systems mainly depend on fossil fuel where the oil price can reach up to four times higher than the prices in the mainland [15]. In recent years, the energy demand is increasing in islands and remote areas, which means that it is not a cost-effective to keep 
relying on fossil fuels. Therefore, standalone HRES or MGs with RES is a promising and sustainable solution to supply the growing population and industries in remote areas and islands with clean and cost effective electrical power [14,16,17].

The intermittent nature of the environment is reflected in the electrical power generated from the RES as most of them come from the environment. For example, wind and solar energy has a strong dependency on the environmental conditions, which is considered as the major drawback of these sources. Nevertheless, this problem can be solved by combining two or more energy sources along with a back-up unit to form a HRES [18]. The combination of RES with complementary characteristics, such as wind and solar, is common in HRESs. Moreover, the integration of energy storage systems (ESSs), such as battery banks, or conventional energy sources, such as diesel generators, makes HRESs capable of providing more economic and reliable supply of electricity to a given application $[18,19]$. However, the high initial cost, increased maintenance cost, and different rates of depreciation are the main challenges associated with these hybrid systems [18]. Moreover, as the HRES design is affected by various factors, such as availability of energy sources and specification of sites, as well as technical, and social constraints [20-22]; they influence the power production arrangements of the system, which increases the total cost of the system [22]. In this context, an optimal sizing combination is a vital factor to achieve higher reliability with lowest costs.

The optimal design of HRESs is a complicated task since the optimal configuration depends on the knowledge of energy sources, technical specifications, environmental conditions, and load profiles [18]. Studies on modeling, configurations, planning, and optimization techniques of HRESs have been conducted for various locations and constraints [3,12,18,20,23-34]. Majority of these studies have used solar and wind hybrid systems as they are efficiently complement each other [28]. In [3], authors have considered the size optimization techniques of on-grid and offgrid solar and wind hybrid systems. In [23], authors have provided a review on optimization and control strategies used for standalone and grid-connected HES. In [27], authors have focused on modelling and size optimization for stand-alone HRESs. The article covers some of artificial single algorithms and classical methods as well. In [29], authors have provided a review on some of the optimization algorithms, operating and control strategies and energy management of standalone and grid-connected hybrid system with the feasibility of the different controllers. In [20], authors provided a review on planning, configurations, modelling and optimization of HRES for standalone applications. However, these articles have not comprehensively addressed all recent single algorithms, hybrid algorithms and software tools with critical comparison of their performances in sizing of standalone solar and wind hybrid systems for remote areas and islands. In [24], authors have provided an overview of some of the sizing algorithms and discussed the optimal sizing process of two HRESs. In [30], authors have focused only on some single artificial algorithms for standalone and grid-connected applications. In [18], authors have provided a review on the use of artificial intelligent algorithms in sizing HRES. In [12], the authors focused on integration configurations, storage system options, sizing methodologies and control and management of standalone HRES. This article provided an overview of some of the single artificial algorithm, classical algorithms and software tools. In [31], authors have provided a review on optimum design of many hybrid combinations covering some of the artificial single algorithms and software tools. In [32], authors have provided a review on multi-objective artificial algorithms considering a few combinations of standalone hybrid systems. In [33], authors have discussed the optimal sizing of different hybrid system combinations for standalone and grid-connected applications which covers some of the artificial and classical sizing methodologies. In [34], authors have focused on hybrid energy systems based on solar, wind and fuel cell energy sources covering only multi-objective optimization algorithms. In [8], authors have focused on the feasibility analysis, control, and modeling of HREs with some artificial optimization techniques.

Even though, the abovementioned literature covers a wide range of sizing optimization, a comprehensive review, putting together the recent single and hybrid size optimization algorithms and software tools with critical comparison of their performances in standalone solar and wind based hybrid systems for remote areas and islands, has not yet been reported. Given the potential of PV-WT HES, especially standalone system for remote and island areas, this article fills this particular gap by presenting a comprehensive review on the recent development in single algorithms, hybrid algorithms and software tools used for optimal sizing of PV-WT HES and assessment parameters including economical, reliability, environmental, and social aspects. Additionally, this article provides the reader with critical comparison between size optimization techniques used for standalone PV-WT HESs with different energy sources and storage systems.

The rest of the paper is structured as follows: Section 2 presents the possible solar and wind configurations and combinations for standalone application, together with a discussion their advantages and limitations. Section 3 explains data input models and the assessments used for optimal design of standalone PV-WT HESs. Section 4 reviews and lists the most recent optimization methodologies for standalone solar and wind energy systems, including single classical algorithms, single modern algorithms, hybrid algorithms and software tools. Moreover, this section presents a performance comparison of optimization algorithms as well. Section 5 presents the findings and discusses the highlighted issues in size optimization and the future trends in size optimization of standalone HRESs. Conclusion drawn from the study are also presented in Section 5.

\section{Combinations of standalone solar and wind HES}

The integration of RESs with other conventional energy sources (CESs) and/or energy storage (ES) devices is common in forming hybrid systems to satisfy a given load demand. For example, PVWT combination provides more reliable power for off-grid and standalone applications compared to individual systems [20]. However, as mentioned above, this particular RES combination requires an energy storage system to be added to alleviate the supply-demand mismatch. Moreover, CESs, such as diesel generators, or modern sources, such as fuel cells, can also be added to the RES to achieve a better energy balance. Fig. 1 shows four possible configurations of such standalone solar and wind HES. Out of these configurations, the dc-coupled connection, shown in Fig. 1 (a), has become popular among many researchers because of the ease of integration and the absence of power quality issues, such as harmonics and reactive power [35-43]. The blackout for ac loads in the event of a failure in the inverter stage is a major drawback of this configuration. To overcome this problem, a number of inverters can be connected in parallel with the main inverter and a fault accommodation mechanism can be employed [44]. However, these solutions increase cost, complexity, weight, and volume. The accoupled system, shown in Fig. 1(b), is a better solution where all the sources are connected to a common ac-bus through interfacing power electronic converters [45-48]. Even if there is a fault in an inverter, the others can continue to supply the entire load or a part of it. Nevertheless, the need for synchronization and inherent power quality issues, such as harmonics and reactive power, are the major disadvantages of this architecture $[12,44]$. The hybrid- 


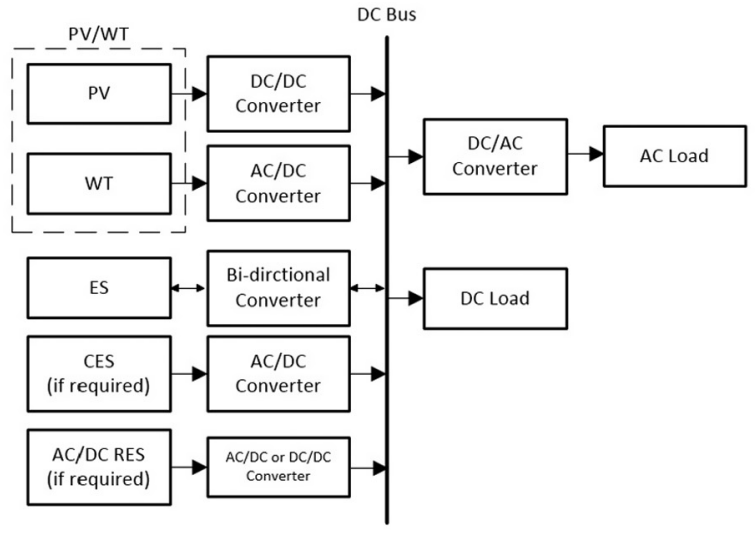

(a) DC-coupled

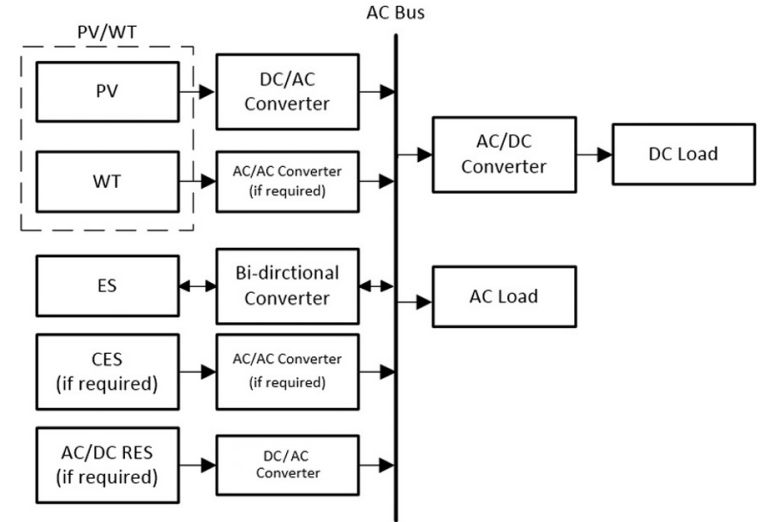

(b) AC-coupled

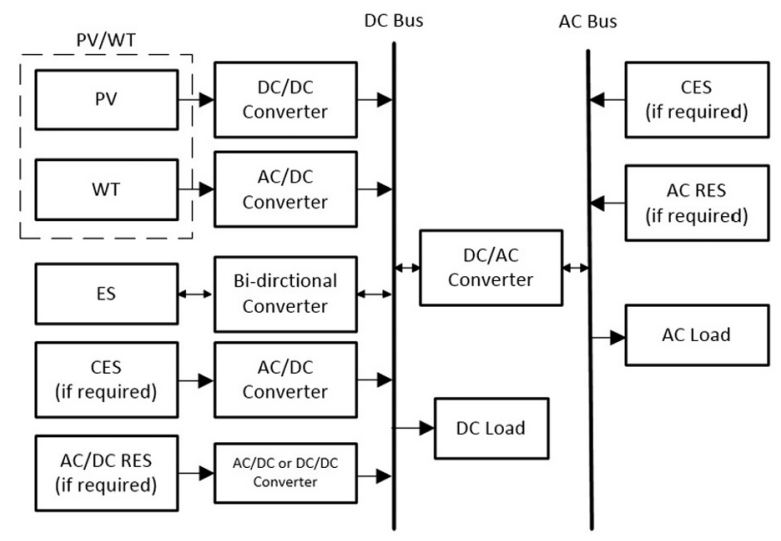

(c) hybrid-copuled option 1

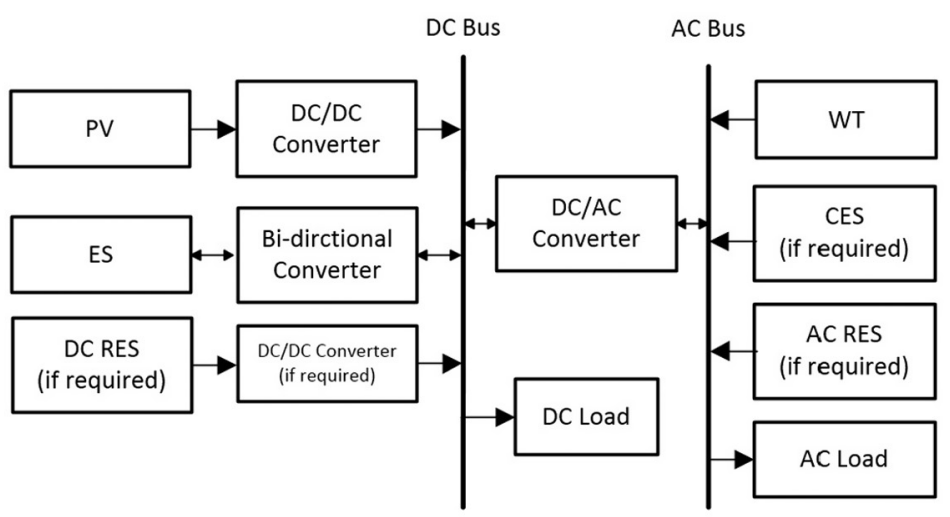

(d) hybrid-coupled option 2

Fig. 1. Standalone PV-WT HES configurations: (a) DC-coupled, (b) AC-coupled, (c) hybrid-coupled option 1 and (d) hybrid-coupled option 2.

coupled systems, shown in Fig. 1(c) and (d), are becoming popular nowadays as they combine the advantages of both dc- and accoupled systems, as well as cost-effectiveness and flexibility to combined loads and sources depending on their characteristics [49-54], [17,55-58]. Moreover, they are more efficient as some of the sources can be connected to the bus directly or with a simple conversion stage $[20,44]$. However, there is no 'one fit all' solution in terms of the combination of RESs and their interconnection; thus, the most suitable combination and architecture should be chosen for the given application and geographical location. Commonly used solar and wind RES combinations are briefly discussed below.

\subsection{Solar and wind}

As solar and wind are strongly correlated to the climate, the generated power fluctuates within a large range and thus the connection to a grid or a back-up device is required to supply the required load. Therefore, the use of a single source, such as wind or solar, for off-grid applications is considered unreliable $[3,59]$. Moreover, wind system alone is found to be uneconomical for some standalone applications [50,57,60-63]. However, WT produces more power than PV system alone, and thus, integrating WTs with PV is important in establishing an eco-friendly HRES for diesel-free generation in standalone applications [64]. In this 
context, solar and wind configuration has more sense in on-grid application [65]. In off-grid application, solar and wind are usually connected with a storage system and/or other energy sources to maintain continued power supply.

\subsection{Solar, wind, and energy storage}

In standalone application, the widely used hybrid solar, wind and energy storage (PV-WT-ES) system combination has proven its reliability to satisfy the load requirements of remote and rural areas. In this combination, PV panels (PVPs) and WTs are connected to a storage device in order to eliminate the power fluctuation of solar and wind resources and to meet the load demand.

The hybrid PV-WT-BS system proved to be the most costeffective combination for islands and remote area compared to PV-BS, WT-BS, and PV-WT hybrid configurations [49,66]. This has been verified through an examination with seven different heuristic optimization techniques [67]. Moreover, recent studies have shown that, PV-WT-BS HES can fully satisfy the load requirements in residential applications in remote and rural areas [49,64,68,69].

Hydrogen tank (HT) is another energy storage option. However, due to high initial costs of such storage system and the need for a fuel cell (FC) to convert the stored energy back into electricity, the hybrid PV-WT-FC energy system is considered to be less costeffective compared to the hybrid PV-WT-BS system [50,70]. Nevertheless, the hybrid PV-WT-FC energy system is more cost effective and reliable compared with hybrid PV-FC and WT-FC systems $[70,71]$. Depending on the area specification such as water availability and rainfall rate, pumped hydro storage system can be a reliable energy storage option. A technical feasibility study by [72] found that a hybrid PV-WT-pumped hydro storage system is capable of supplying the full load demand in a remote area without grid support. Another storage option is the super-capacitor which has a high power density and high charge/discharge efficiency [73]. However, they have not been widely used because of their high cost and limited energy capacity compared to battery or other competitive energy storage technologies.

\subsection{Solar, wind, and other renewable energy source and storage}

In this combination, all energy sources combined with PV and WT are RESs, including FC, hydro generator (HG), biomass (BM), and biogas (BG). The main advantage of this combination is its minimal or zero carbon emissions. Furthermore, the use of more RES to compensate conventional sources increases job creation (JC) as it increases the manufacturing and installation rates of renewable systems [74]. FC system, including electrolyzer (EL) and HT, provides an environmental friendly and high efficiency energy system $[53,75]$. However, the initial cost of this system is relatively high [50]. Therefore, integrating FC and HT with a hybrid solar and wind system can effectively reduce the installation costs of FC and HT [76]. Out of the above-mentioned RES combinations the PV-WT-FC system with HT storage found to be more common as it provides a cost-effective solution compared to PV-FC and WTFC systems [39,50,53,57,71].

In certain locations, especially in rural and remote areas, the use of an integrated renewable energy (IRE) system by utilizing as much renewable sources as possible at the site to produce electricity can provide more cost-effective option than introducing CES. For example, for villages and areas where biomass resources are available, PV-WT-BM-BS can provide a more cost-effective option than using CES such diesel generator [17] .Some other hybrid combinations, such as PV-WT-BG-BM-BS and PV-WT-BG-BM-HG-BS systems are also capable of providing cost-effective and reliable systems for remote areas and villages [74,77].

\subsection{Solar, wind, and conventional energy source and storage}

In this combination, PV and WT are combined with CES and ES system. Mostly, in this combination, diesel generator (DG) and battery storage (BS) are coupled with PV and WT. Although this configuration produces some emissions due to the use of CES, it is widely used in standalone application as it is more reliable in supplying the load demand. The use of BS is more cost-effective than totally relying on DG as a back-up source of PV and WT [78]. Therefore, the PV-WT-DG-BS HES is common in standalone applications as it can ensure continuity of power supply $[61,79,80]$. Depending on the load demand and the size of the battery, the DG can be considered as a back-up power source. The DG operates only when PV power, WT power, and BS back up are not able to supply the load demand [81]. This reduces the operating hours of the DG and thus reduces the emissions [80,82]. Therefore, PV-WT-DG-BS HES is more cost-effective and reliable for standalone application than PV-WT-DG HES $[38,79,83]$.

\subsection{Solar, wind, and other renewable and conventional energy source and energy storage}

In this combination, PV and WT are combined with RESs, CES and ES. This combination is not widely implemented as it has a high initial cost and maintenance cost. However, in some locations, this combination provides a cost-effective system more than other configurations depending on the site's specifications, such as the availability of RESs, transportation of fuel, and load demand. Some studies proposed combinations such as PV-WT-DG-FC-BS-HT [84], PV-WT-DG-hydro generator (HG)-BS [85,86], PV-WT-DG-FC-BS-HT [40], PV-WT-DG-FC-bio-diesel(BD)-BS [87], PV-WT-HG-BS [88], and PV-WT-BD-HG-BS [89].

\section{PV-WT HES requirements and assessment parameters}

\subsection{Data input}

Solar irradiance and wind speed data affects the size optimization sizing results. The accuracy of the optimization results improves when the forecasted data is used instead of the data of the past years [90-93]. Moreover, the peaks of solar irradiation and wind speed influence the size optimization results by increasing the initial and operation cost values [46]. Therefore, implementing estimation and forecasting techniques to obtain a forecasted data improves the accuracy of the size optimization algorithm results. Hocaoglu et al. [93] investigated the effects of past years' solar irradiation data on the sizing of HES, and found that previous years' data cannot produce a similar loss of load probability (LLP) for a future year. Gupa et al. [90] investigated the use of historical and forecasted data on the optimization results. The authors implemented a back propagation trained artificial neural network (BPANN) for forecasting wind speed and solar irradiance. The study found that the forecasted weather data improves the optimization results. Sinha and Chandel [94] used artificial neutral network (ANN) to predict solar and wind data, and found that the predicted data by ANN are close to the measured and estimated data. Rajkumar et al. [95] applied an Adaptive Neuro-Fuzzy Inference System (ANFIS) to model a PV module and WT and thereby generate solar radiation, wind speed, and temperature datasets. To predict the output power of PV and WT, weather-generated data are used to train the neuro-fuzzy model. Nogueira et al. [96] calculated the hourly generated wind and solar power using a statistical model based on the Weibull and Beta probability density function (pdf). Khatod et al. [97] also had applied Beta and Weibull distributions for predicting the solar 
radiation and wind speed. Ekren and Ekren [98] used the ARENA simulation software to predict the wind speed, solar radiation, and electricity consumption distributions at a telecommunication base station in order to design a HES to supply it. The authors in [37] used autoregressive moving average models (ARMA) to model the variation of solar irradiance and Weibull distribution to model for wind speed in Kent, UK. Zhao and Yuan [82] obtained the one year hourly wind speed data through HOMER according to Weibull distribution and local meteorological data collected, and obtained the one year hourly solar radiation data on horizontal plane by using solar radiation law. Azimi et al. [99] developed a hybrid forecasting method consists of a time series analysis, a novel cluster selection algorithm and multilayer perceptron neutral network (MLPNN) to predict solar radiations. Chen [100] estimated the WT and PV power generation based on previous hourly solar irradiation, wind speed, and temperature data. Vasilj et al. [101] presented an estimation model based on Monte Carlo simulation (MCS) to estimate the power uncertainties and associated balancing and reserve power requirements of hybrid PV-WT system due to solar irradiation and wind speed uncertainties. The model uses production simulation for solar radiation and wind speed and forecast error simulation for wind speed, PV power, and load forecast error.

Not only the site energetic potential (solar radiation and wind speed) but also the load profile constitution affect the optimization results [79]. The load profile can be accomplished through measurement and load research surveys. If the load profile is not available, synthetically generated load profiles can be used. Several studies have been conducted on load profile estimation using different estimation and prediction methods [102-109]. ANN is used in [110] to generate a load profile based on its typical meteorological year 2 (TMY2) weather data. The ANN model was trained with the TMY2 weather data and the load profile data of neighboring regions is used to estimate a residential load for Gujarat, India. Cross-entropy (CE) is a non-parametric estimation method for density probability. This method has been used by [111] to estimate the pdf of the user energy consumption starting from measured data.

Given the fact that most of standalone HESs are used in remote and rural areas, the load profile data is unavailable in many cases. Therefore, increasing the research on improving the accuracy of estimation and forecasting approaches to obtain more accurate load profile data is necessary so as to increase the accuracy of the size optimization results.

\subsection{Assessment parameters of PV-WT HES}

There are various indicators reported in literature to assess HRES. These indicators can be broadly classified into four categories, namely: economical, reliability, environmental, and social assessments. These parameters evaluate the availability and feasibility of HES to help in the design and construction of an optimal system for a given application. Economical assessment is a main factor in determining the desirable minimum initial, maintenance, replacement, and any other future costs of a HRES. The reliability assessment evaluates the hybrid system's ability to ensure the cohesion of HRES in order to satisfy load demand. Environmental assessment evaluates the amount of $\mathrm{CO}_{2}$ and other obnoxious emissions produced by the system throughout a given period of time. Social assessment evaluates the capability of the HES to produce energy for increasing the human development index (HDI). Moreover, it evaluates the social acceptance of installing hybrid system and job creation. The summary of the assessment parameters for standalone PV-WT HES is illustrated in Table 1.

\section{Size optimization techniques}

Size optimization techniques can be classified into classical techniques, modern techniques and software tools. Classical techniques use iterative, numerical, analytical, probabilistic, and graphical construction methods [3]. These methods utilize differential calculus in deriving the optimum solution [20]. Modern techniques use artificial and hybrid methods [3,23]. These methods can determine the global optimum system and has better convergence and accuracy in finding a set of optimal solutions [3,25]. The third size optimization approach for HES sizing include computer software tools. The most widely used software tool in size optimization for standalone PV-WT HES is Hybrid Optimization Model for Electric Renewables (HOMER) [23,112]. Another software, named Improved Hybrid Optimization by Genetic Algorithm (iHOGA) has been used in sizing optimization for standalone PV-WT HES [23]. Fig. 2 shows the recent size methodologies for standalone PV-WT HES.

As the HRES design is complex due to the uncertainties associated with renewable resources and other technical factors and the constraints associated with the site location and system components. Classical techniques are not efficient in solving such complex problems. Therefore, in the last decade, modern techniques that are based on meta-heuristics algorithms have extensively been used $[3,113]$.

Sizing optimization methodologies can use either a single objective optimization (SOO) function or multi objective optimization (MOO) functions. SOO is used to find the optimum solution corresponding to the minimum or maximum value defined by the SOO function. In contrast, MOO combines two or more individual objective functions to determine a set of trade-off solutions, which allow decision makers to select the most suitable solution based on the problem requirements [32]. In this context, the use of MOO provides more efficient results as it finds the global optimum Pareto-set solutions, thereby improves the costeffectiveness and reliability of HES combination compared to the SOO $[39,42]$.

Most classical techniques use single algorithms with SOO function. Modern techniques use single and hybrid algorithms to solve SOO or MOO problems. Hence, modern methods are more flexible in dealing with complex optimization problems, and they provide more accurate results. An overview of the optimization techniques discussed in this paper is shown in Fig. 3.

\subsection{Single algorithm}

Single algorithms including classical and artificial techniques used to solve the size optimization for PV-WT HES are reviewed in the following sub-sections and the summary of each technique is presented in Table 2.

\subsubsection{Classical techniques}

A limited number of studies have recently been carried out using classical methods in size optimization of standalone PV-WT HES. Most of these studies are conducted using iterative algorithms [47,50,114-121]. Hosseinalizadeh et al. [50] implemented an iterative algorithm to optimize a standalone PV-WT-FC-BS-HT HES in terms of minimizing the system's total COE for four different regions in Iran. The authors used a proton exchange membrane fuel cell (PEMFC) as a back-up source to the battery storage system rather than directly supplying the load. The PEMFC operates when the charge level of the battery bank drops below the allowable level. The authors of [50] have assessed the reliability of the HES by using LOEE and LOLE as assessment parameters. In this study, it has been assumed that the value of LOLE parameter must be less 
Table 1

Summary of economical, reliability, environmental, and social assessment parameters for PV-WT HES.

\begin{tabular}{|c|c|c|c|}
\hline Assessment & Indicator & Description & Ref. \\
\hline \multicolumn{4}{|l|}{ Reliability } \\
\hline & Loss of Power Supply Probability (LPSP) & The probability of unmet load over the total energy produced & {$[67,72,83,90,95,164,165]$} \\
\hline & Loss of Load probability (LOLP) or (LLP) & The ratio of annual energy deficits to annual load demands & {$[120,146]$} \\
\hline & $\begin{array}{l}\text { Loss of Load Risk (LOLR) or loss of load } \\
\text { expectation (LOLE) or loss of energy } \\
\text { expectation (LOEE) }\end{array}$ & $\begin{array}{l}\text { The average number of hours for which the system load is expected to exceed } \\
\text { the available generation capacity }\end{array}$ & {$[49,50,97]$} \\
\hline & Unmet Load (UL) & $\begin{array}{l}\text { The amount of power shortage at load that exceeds the amount of generated } \\
\text { energy from all energy sources and stored energy in all storage devices }\end{array}$ & {$[72,90,113]$} \\
\hline & $\begin{array}{l}\text { Deficiency in Power Supply Probability } \\
\text { (DPSP) }\end{array}$ & The amount of power shortage at each hour & [37] \\
\hline & $\begin{array}{l}\text { Expected Energy not Supplied (EENS) or } \\
\text { Energy not supplied (ENS) }\end{array}$ & The amount of load energy not supplied during a period of time. & {$[49,54,97,140]$} \\
\hline & Energy Index of Reliability (EIR) & The ratio of expected energy not supplied to the load demand & {$[140]$} \\
\hline & Equivalent Loss Factor (ELF) & The ratio of the effective forced outage hours to the total number of hours & {$[39,52]$} \\
\hline & Net Dump energy (D) & The total dump energy produced from RES & [55] \\
\hline & Total Energy Deficit (TED) & $\begin{array}{l}\text { The ratio of energy not supplied to the consumer when was requested on the } \\
\text { total energy required }\end{array}$ & {$[118]$} \\
\hline & Wasted Renewable Energy (WRE) & The energy produced by RES that cannot be stored in the storage system & {$[166]$} \\
\hline & Renewable energy penetration (REP) & $\begin{array}{l}\text { The ratio of total energy generated from RESs to the total energy demand of } \\
\text { the load for a year }\end{array}$ & [47] \\
\hline & Final Excess of Energy (FEE) & $\begin{array}{l}\text { The difference between the accumulated electrical energy of the battery and } \\
\text { initial electrical energy of the battery }\end{array}$ & {$[35]$} \\
\hline & Loss of Energy Probability (LEP) & $\begin{array}{l}\text { The ratio of the wasted energy by the scheduler model and the total load } \\
\text { demand during a year }\end{array}$ & {$[42]$} \\
\hline & Energy Fluctuation Rate $\left(\mathrm{K}_{\mathrm{l}}\right)$ & $\begin{array}{l}\text { The fluctuation rate of the entire system indicating that the optimal system } \\
\text { output energy is matching the load demand to reduce the energy impulse of } \\
\text { the system, increase the power supply quality and reduce the work load of the } \\
\text { scheduler model }\end{array}$ & {$[42]$} \\
\hline & Risk state probability $P(R)$ & $\begin{array}{l}\text { The percentage of time when generation is inadequate to supply load within a } \\
\text { study period }\end{array}$ & {$[132]$} \\
\hline & Percentage of healthy state probability $P(H)$ & $\begin{array}{l}\text { The percentage of time for which the system has adequate reserves to satisfy } \\
\text { laid down reverse criteria within a study period }\end{array}$ & {$[132]$} \\
\hline \multicolumn{4}{|l|}{ Economic } \\
\hline & $\begin{array}{l}\text { Net Precent Cost (NPC) or total precent cost } \\
\text { (TPC) or Net present value (NPV) or total } \\
\text { cost (TC) }\end{array}$ & $\begin{array}{l}\text { The total investment, maintenance, operation and replacement costs } \\
\text { throughout the life time of the system }\end{array}$ & {$[21,78,118,167]$} \\
\hline & Total investment cost (TIC) & $\begin{array}{l}\text { Include capital cost, installation cost, annual operation and maintenance cost } \\
\text { and replacement cost throughout the system lifetime }\end{array}$ & {$[51]$} \\
\hline & Life Cycle Cost (LCC) & $\begin{array}{l}\text { The costs of system operation throughout lifetime. Does not include } \\
\text { manufacturing and disposal costs }\end{array}$ & {$[54,55,66,131]$} \\
\hline & $\begin{array}{l}\text { Levelised cost of energy (COE) or (LCE) or } \\
\text { (LCOE) }\end{array}$ & $\begin{array}{l}\text { The ratio of the costs and total energy consumed by the load throughout the } \\
\text { lifetime of the system }\end{array}$ & {$[45,121,132,167,168]$} \\
\hline & $\begin{array}{l}\text { Total annual cost (TAC) or annual system } \\
\text { cost (ASC) }\end{array}$ & $\begin{array}{l}\text { The summation of capital costs, replacement costs, operation costs and annual } \\
\text { maintenance costs }\end{array}$ & {$[53,82]$} \\
\hline & Cumulative savings & $\begin{array}{l}\text { Sum of money which is saved by hybrid system for a period of time because of } \\
\text { fuel saving }\end{array}$ & [147] \\
\hline \multicolumn{4}{|l|}{ Environment } \\
\hline & Total $\mathrm{CO}_{2}$ Emissions (E) or fuel emissions & $\begin{array}{l}\text { The total amount of } \mathrm{kg} \text { of } \mathrm{CO}_{2} \text { emissions produced by the system throughout a } \\
\text { period of time }\end{array}$ & {$[55,82,83,113,169]$} \\
\hline & Embodied Energy (EE) & $\begin{array}{l}\text { The energy that the hybrid system does not consume during its use, but } \\
\text { involves the consumption of non-renewable primary energy for components } \\
\text { manufacturing. In other words, it is the energy required by all the activities } \\
\text { associated to a production process }\end{array}$ & [131] \\
\hline & Life cycle assessment (LCA) & $\begin{array}{l}\text { The assessment of all the stages of a product's life of hybrid system } \\
\text { components including emissions associated with material manufacturing, } \\
\text { procurement and transportation }\end{array}$ & {$[80]$} \\
\hline \multicolumn{4}{|l|}{ Social Criteria } \\
\hline & Human Development Index (HDI) & $\begin{array}{l}\text { HDI is a country development indicator that takes into account life expectancy } \\
\text { at birth, expected years of schooling and gross national income per capita. It } \\
\text { depends on the consumption of electricity, so the extra energy that can be } \\
\text { supplied by the hybrid system can improve the HDI index }\end{array}$ & [21] \\
\hline & Job Creation (JC) & $\begin{array}{l}\text { The jobs created of manufacturing, installation and O\&M of the hybrid system } \\
\text { components. The number of jobs created by a hybrid system depends on the } \\
\text { combination of components, so more jobs created better hybrid system } \\
\text { combinations }\end{array}$ & {$[21,74]$} \\
\hline & Social Cost of Carbon (SCC) & $\begin{array}{l}\text { Imposed by incorporating an additional cost component. It acknowledges } \\
\text { effect of emissions from DGs on society }\end{array}$ & {$[132]$} \\
\hline & Socio-demographic factor & $\begin{array}{l}\text { It describes the energy consumption pattern of a household load in a certain } \\
\text { location. This factor can be used in sizing HES by estimate how a one class of } \\
\text { user responds to the demands from another class of user }\end{array}$ & [36] \\
\hline
\end{tabular}




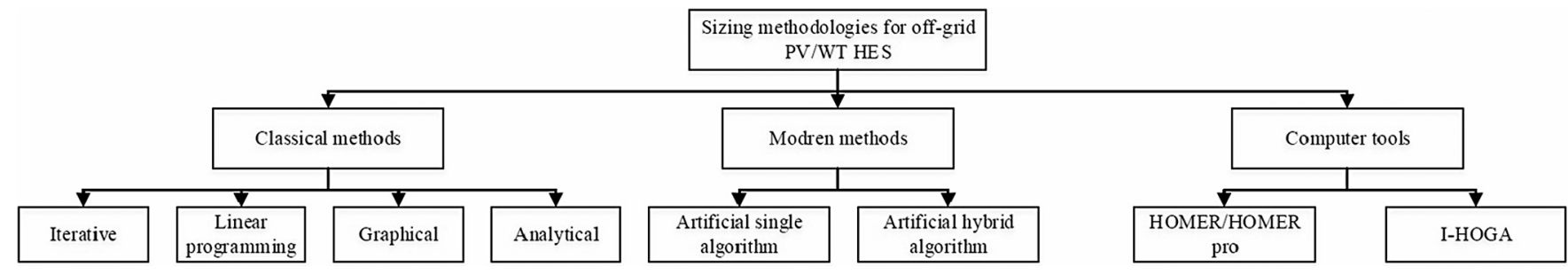

Fig. 2. Recent size optimization methodologies for standalone PV-WT HES.

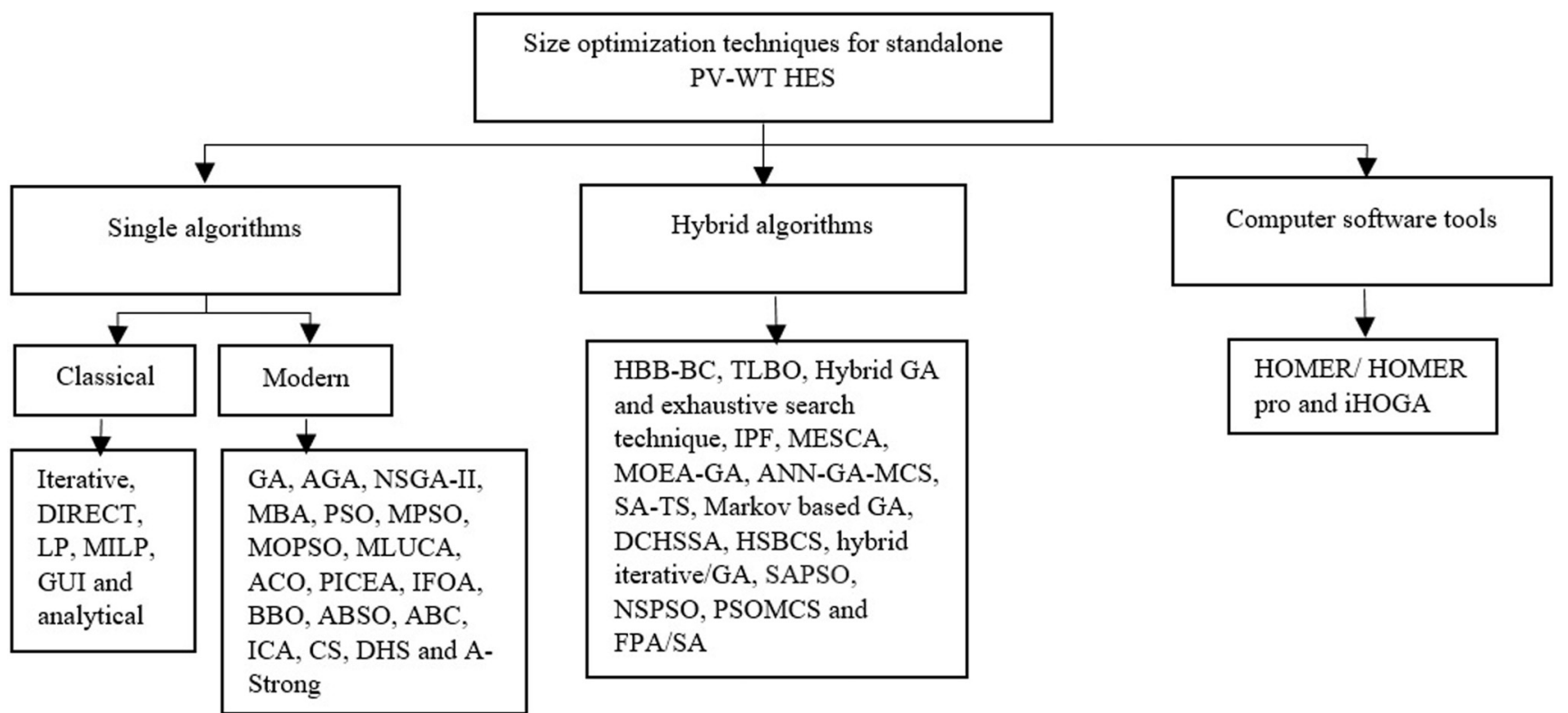

Fig. 3. Overview of the size optimization techniques discussed in this paper.

than $2 \%$ in order to achieve a reliable system. The study found that the PV-WT-BS HES is more economical and reliable without the FC system. Smaoui et al. [76] proposed an optimization methodology based on iterative technique to optimize the size of a standalone PV-WT-FC-HT HES in order to supply a desalination unit for the Kerkennah Island in South Tunisia. The optimization algorithm was implemented in two parts by calculating the FC installed and EL installed powers, and the proposed combination part which is tested to assess technical performance. The main objective of the optimization is minimizing the total capital cost of the system. The study found that the proposed HES was able to meet the load demand, and the complementary characteristics of the hybrid combination of PV and WT reduced installation costs due to decreased storage requirements. Bhuiyan et al. [47] proposed an enumeration-based iterative algorithm to optimize the component sizes for an islanded micro-grid for off-grid communities. The system consists of PV, WT, BS, and DG. The main optimization function of minimizing the LCC is used to assess the feasibility of the HES combination. The LPSP and REP are used to assess the reliability of the system by exanimating the effect of these parameters on LCC. The proposed algorithm provided lower LCC compared to HOMER. Additionally, the study found that LCC value is minimized without seasonal variations and high REP. Moreover, the LCC value is reduced when LPSP percentage is decreased.

DIRECT algorithm is an efficient deterministic algorithm in finding the global optimum of several problems. This algorithm is used in [79] to determine the optimum system configurations that the system total cost is minimized while the availability of energy is guaranteed. In this study, the reliability of the system is assessed by analyzing the battery SOC and the power balance between generation and demand. Furthermore, the study found that PV-WT-DG HES system is found to be techno-economic in meeting the energy demand of remote consumers.

A few studies have recently used linear programming (LP) in optimizing the size of standalone HES with PV and WT $[96,122]$. Nogueira et al. [96] proposed a methodology that uses LP to size and simulate a standalone PV-WT-BS HES for a remote rural area by minimizing the TC of the system while satisfying the load demand. The reliability of the system is assessed by using the LPSP parameter. The optimal sizing of the system is performed with six different scenarios, each with varying lengths of critical periods of predetermined amounts of consecutive hours and LPSP. Malheiro et al. [45] implemented the deterministic optimization, mixedinteger linear programming (MILP), to find the optimal mix between PV-WT-BS-DG by minimizing LCOE over a lifetime of 20 years. The optimal system was achieved with $90.0 \%$ of renewable fraction. Ferrer-Marti et al. [123] proposed a methodology using MILP and exact solve procedure with taking into account the energy demand at the consumption points and the energy resource maps to find the optimal size and location of the hybrid PV and WT system components. The objective function of the optimization is to minimize the initial system cost which is used as the parameter to assess the system. The study found that the optimal location, in addition to optimal size, reduces the initial investment costs.

Gan et al. [46] used a graphical user interface (GUI) to optimize the size of a hybrid PV, WT, BS, and DG system considering the peaks and troughs of wind speed and solar irradiance over a year. 


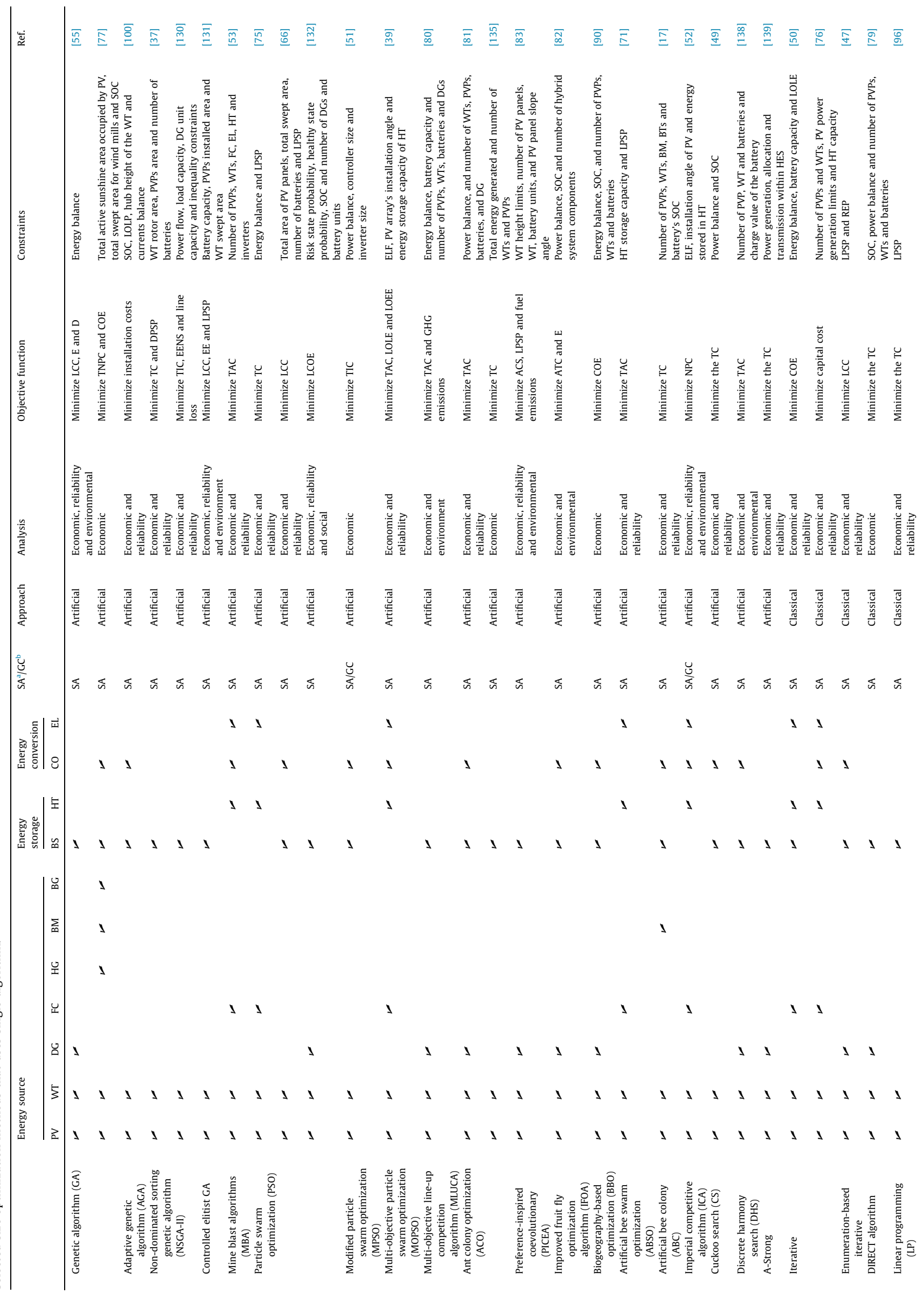




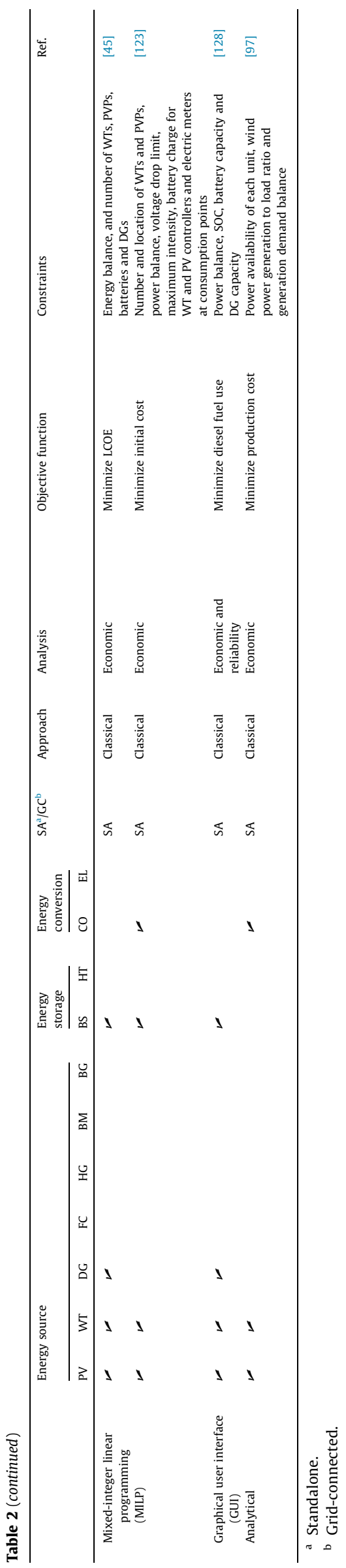

The objective function of this optimization is to minimize the use of diesel generator. The decision of turning on the diesel generator optimally is considered as the economical assessment parameter while power balance and SOC are used to assess the reliability of the system. The proposed method used the measured annual hourly solar irradiation and wind speed to simulate the real time operation of the hybrid system. The study found that the peaks of solar irradiation and wind speed affect the size optimization of the results.

Analytical methods are based on mathematical analysis, theoretical analysis and calculations. These methods use computational models to find the HES size as a function of its economic feasibility [25]. In these methods, a series of logical steps need to be defined and followed in order achieve the exact solution. In contrast, in numerical methods, the problem does not have a specified procedure to follow in order to achieve a set of approximated solutions [124,125]. Therefore, analytical methods required more computational time than numerical methods [33]. These methods have not been widely used in the size optimization of standalone HES consisting of PV and WT in recent years [97,126]. In [97], the authors implemented an analytical method to minimize the production cost of PV-WT hybrid autonomous system in Kandla, India. The authors validated the results obtained from the proposed analytical method by comparing it to the results obtained by MCS. In the proposed analytical method, the amount of metrological data input is less in comparison to MCS. Therefore, the proposed analytical method provided low computational burden with relatively less time compared to MCS.

\subsubsection{Artificial technique}

Artificial techniques have been implemented by several researches to attain the optimal size of standalone HES. These techniques can handle multiple objective problems and provide an optimal solutions set. The most recent artificial single algorithms applied for standalone PV-WT HES are discussed below.

Genetic Algorithm (GA), an evolutionary heuristic search algorithm, is one of the most powerful optimization algorithm. Numerous studies have implemented GA in finding the optimal sizing of HRE system [48,55,77,127-129]. Ogunjuyigbe et al. [55] used GA for the optimal sizing and allocation of HES in standalone mode. In this study, the authors investigated five different combinations for residential load, as well as the possibility of using small aggregated diesel generators instead of a single big-sized diesel generator. In this study, LCC, net dump load (D) and total $\mathrm{CO}_{2}$ emissions are used to assess the system's economical, reliability and environmental aspects respectively. The study found that PV-WT-Splitdiesel-BS HES is the most optimal combination in terms of the minimum LCC, COE, net dump energy, and $\mathrm{CO}_{2}$ emissions. Additionally, the study found that the use of aggregated split diesel generators rather than a single big-sized diesel generator is more costeffective. Rajanna and Saini [77] used GA for the optimal sizing of integrated renewable energy sources (IRES) considering several RESs and a battery storage system for four different zones in Karnataka, India. The study aims to find the optimal scenario combinations among three different scenarios based on minimizing TNPC and COE. The optimal combination of the system is found based on the two economical assessment parameters TNPC and COE. The study found that two scenarios consist of PV, WT, and BS with other energy sources are the most cost-effective. Adaptive GA (AGA) is used by [100] to optimize the size for a standalone PVWT-BS HES because of its improved adaptability with computational simplicity to solve such non-linear problem. In this study, WT and PV generation powers were estimated based on previous hourly solar irradiation, wind speed, and temperature data for the Wuchi and Orchid islands in Taiwan. The objective function of the optimization is to minimize the total installation cost of 
the hybrid system. The reliability of the system is assessed by using LOLP. The optimal capacity of the standalone system was achieved for both locations in terms of total cost and reliability. Another improved version of GA, called non-dominated sorting genetic algorithm (NSGA-II), is used by [37,130] and provided promising results in solving MOO problems. Kamjoo et al. [37] applied the NSGA-II algorithm to optimize a standalone PV-WT-BS HES combination for a household load in Kent, UK. The economic and reliability objective functions of the optimization are to minimize TC and DPSP. Owing to the uncertainties arising from renewable resource which affect the DPSP value, the study used chance constrained programming (CCP) with NSGA-II to estimate the DPSP value. The proposed method provided more conservative set of solutions compared to the usage of Monte Carlo Simulation (MCS). Another study by [130] used NSGA-II in finding the optimal allocation for PV-WT-BS HES in MG. In this study finding the minimums of TIC, EENS and the losses of the line are used as economic and reliability objective functions. The results of this study revealed that the proposed hybrid system is optimized in terms of minimum investment cost and maximum reliability when combined with MG. A variant of NSGA-II, called a controlled elitist GA, is utilized by [131] to obtain the optimal combination of standalone PV-WT-BS HES for a residential application. A triple multi-objective function combination LCC, LPSP and EE is used in this study. Furthermore, the optimal size was achieved considering the economical, reliability and environmental assessment parameters.

Fathy [53] implemented mine blast algorithm (MBA) to find the optimal sizing for a HRES in terms of minimizing the ATC for Helwan, Egypt. The reliability of the system is assessed by ensuring the power balance between generation and load. The author used a real measured data of solar radiation, wind speed, and temperature to investigate the optimal size for three different combinations. The most cost-effective combination was found to be PV-WT-FC system.

Particle swarm optimization (PSO) is one of the most popular heuristic algorithms in solving non-linear optimization problem because of its simplicity, ease of implementation and fast convergence. Paliwal et al. [132] implemented PSO to find the optimal combination of PV, WT, DG, and battery units in terms of reliability $(P(R)$, and $P(H)$ ), social (SCC), and economic (LCOE) assessment parameters. As result, the reliability parameters are met with less storage units by using ones with large storage capacities, which reduced replacement costs. Moreover, the integration of RES with DGs reduces SCC. Sanchez et al. [75] used PSO to optimize the size for standalone PV, WT, FC, and HT for the remote residential load in Chetumal, Mexico. The objective function of the optimization is to minimize the system TC while ensuring the reliability of the system. LPSP parameter is used to assess the reliability of the system. The optimal size of components was found in terms of the lowest TC for 20 years. Askarzadeh and Coelho [66] used PSO and some of its variants to find the optimal combination among PV, WT, and BS for a remote area located in Kerman, Iran. In this study, minimizing the LCC is used as the economic objective function while LPSP is used to assess the reliability of the system. The results showed that the adaptive inertia weight-based PSO, which has a better balance between global and local search and resultant elimination of premature convergence, provided minimal LCC compared to the original PSO and its other variants. A similar study is conducted by [41] to determine the optimal renewable mix for a remote area in Iran in terms of minimizing TAC. The authors concluded that PSO-CF produces more promising results compared to PSO, other PSO variants, and other artificial algorithms. Hassan et al. [51] proposed a modified PSO (MPSO) to find the optimal combination of both standalone PV/WT/BS HES system and grid connected PV-WT HES. The economic objective function of this optimization exercise was to minimize the TIC of the system. The proposed algorithm provided the optimum TIC for the standalone mode. Bghaee et al. [39] used multi-objective PSO (MOPSO) to optimize the economical and reliability aspects of HES comprised of PV, WT, FC, and HT for 20 years. The main economic and reliability objective function of the optimization is to minimize the TAC, LOLE and LOEE of the system. The reliability of each component directly affects the annual cost of the entire system. Therefore, by examining the effect of each component outage on the whole system's reliability and cost authors optimized the sizing for different cases. Borhanazad et al. [133] implemented MOPSO to find the optimal sizing for a PV, WT, DG, and BS micro-grid for three different locations in Iran. The optimal combination is achieved by minimizing COE and LPSP. Safar et al. [134] used PSO to optimize the size for a standalone PVWT-BS-FC system to make the HES economical with high reliability. The study used fuzzy logic controller to regulate energy flow on HES. The membership functions of FLC are then optimized by PSO. The study found that a well optimized FC system improves the life time of the batteries by reducing the variation in SOC.

Shi et al. [80] used a multi-objective line-up competition algorithm (MLUCA) to optimize the size of a standalone PV-WT-DGBS HES in terms of economic and environmental aspects. The economic and environmental objective function of the optimization is to minimize TAC and GHG emissions of the system. The authors first introduced an improved power management strategy to improve the battery utilization and then implemented MLUCA algorithm to find the best combination of components that can satisfy the load demand. The authors found that the proposed algorithm incurred high cost and an optimum combination in terms of minimum GHG emissions.

Ant colony optimization (ACO) is a metaheuristic optimization technique with inherent capability of parallel computing, such that it can solve complex problems with dynamic behavior. Suhane et al. [81] applied ACO to find the optimal mix of PV, WT, BS, and DG in terms of minimizing TAC for a village in India. The reliability assessment parameter LCOE, which has good overall performance with only $2 \%$ unmet load, is found to be much less than the COE per unit for DG. Fetant and Khorasaninejad [135] employed ACO for continuous domains $\left(\mathrm{ACO}_{\mathrm{R}}\right)$ based on integer programming to find the optimal mix of PV, WT, and BS system by minimizing TC (capital and maintenance). The reliability of the system is assessed by ensuring the power balance between generation and load. The minimum TC achieved for wind standalone system is \$5652.65, followed by the hybrid of 20 PVPs, 2 WTs, and 9 battery units at $\$ 6429.19$. In this study, $\mathrm{ACO}_{\mathrm{R}}$ provided the optimal solution of the total costs with lower convergence iterations and time compared to GA and artificial bee colony (ABC).

Preference-inspired coevolutionary algorithm (PICEA) that uses goal vectors is a search technique which can solve complex multiobjective optimization problems. The idea of this algorithm is to coevolve multiple sets of preferences during the optimization process in order to provide different Pareto front subsets to the decision maker [136]. Shi et al. [83] implemented PICEA to design the size of standalone HES. The proposed methodology is developed to minimize ACS, LPSP, and E of a standalone PV-WT-BS-DG HES. The optimal combination is achieved with 0\% LPSP and ACS of $\$ 8200.79$.

Fruit fly optimization algorithm (FOA) is a heuristic evolutionary computation method used in finding global optimization. Zhao et al. [82] used an improved FOA (IFOA) algorithm for the optimization design of standalone PV-WT-BS-DG HES in Dongan island in China. An economic and environmental multi-objective function is utilized in the optimization that combines TC and E as assessment parameters. The study aim to find the optimal size combination in terms of minimal TC and E. The study found that with less number of WTs and battery units, better total cost, and increased $\mathrm{CO}_{2}$ emissions can be achieved. The most economical system in 
terms of TC produces $5087.39 \mathrm{~kg} /$ year of $\mathrm{CO}_{2}$ emissions, whereas the least economical combination is free-carbon emission.

Biogeography-based optimization (BBO) is a population-based evolutionary method. Few studies that use BBO to optimize the size of HES consisting of PV, WT, and other sources and storages have been conducted [90,137]. Gupa et al. [90] implemented BBO to find the optimal combination of a standalone PV- WT-DG-BS HES in terms of minimizing COE. The power balance between generation and demand is considered to ensure the reliability of the system. In this study, ANN is used to forecast weather data. Optimal sizing is achieved using the forecasted data, as these improve the quality of optimization results more than the previous year's data.

Artificial bee swarm optimization (ABSO) is a metaheuristic algorithm that employs different types of bees to amend their positions in escaping local optima and finding a global solution. Maleki and Askarzadeh [71] implemented ABSO to find the optimal mix of HES components in terms of the minimum TAC. In this study, LPSP is used to assess the reliability of the system. The optimization results proved that PV-WT-FC is the most cost-effective system with $0 \%$ LPSP $_{\max }$.

Singh et al. [17] used artificial bee colony (ABC) to determine the optimal combination of a PV-WT-BM-BS HES in order to achieve a cost-effective and reliable HES for an isolated small village in Patiala in Punjab, India. The economic objective function of the optimization is to minimize the ASC of the system. The reliability of the system is assessed by finding the effect of failure of any generation unit of the system. The authors implemented $A B C$ and compared its performance with HOMER and PSO. The optimal combination is achieved with an annual system cost of $\$ 63,006$.

Imperial competitive algorithm (ICA) is an evolutionary algorithm that can solve non-linear and multi-objective problems. Gharavi et al. [52] implemented ICA to optimally design a standalone and grid-connected HES that includes PV-WT-FC and electrolyzer (EL) while considering reliability (LOEE, LPSP and ELF), economic (NPC), and environmental (E) assessment parameters. The optimization method is implemented by first solving the multi-objective function using fuzzy logic and then employing ICA for optimization purposes. The study found that the gridconnected system is more cost-effective, but it has high $\mathrm{CO}_{2}$ emission levels compared with standalone mode.

Cuckoo search (CS) is a metaheuristic algorithm that can address complex and multi-objective optimization problems. Sanajaoba and Fernandez [49] applied CS for optimal sizing relative to the TC of three different combinations for a remote area in India. The reliability is assessed by quantifying LOLE. The study found that the standalone PV-WT-BS HES provided the most costeffective and reliable system than other HES combinations. Additionally, the study revealed that CS provides better-quality solutions compared with other evolutionary algorithms in HES sizing.

Maleki and Askarzadeh [138] used discrete harmony search (DHS) to find the optimal size combination system among PV, WT, BS, and DG in terms of minimizing TAC and total emissions. The total emission parameter consist of the total emissions of $\mathrm{CO}_{2}, \mathrm{SO}_{2}$ and $\mathrm{NO}_{2}$. The system components are modeled based on the measured solar irradiation and wind speed data for Rafsanjan, Iran. The authors found that the optimal combination is WT-DG-BS which is followed by the PV-WT-DG-BS system.

Stochastic trust-region response-surface (STRONG) method is a meta-model algorithm based on response surface methodology (RSM) and the classic trust-region method. Chang and Lin [139] implemented A-STRONG method, which modified the STRONG method coupled with MCS to find the minimum TC for a standalone PV-WT-BS-DG HES. The balance between power generation and demand is used to assess the system reliability. The optimal combination is achieved by considering power generation allocation and transmission.

\subsection{Hybrid algorithm}

Artificial single-optimization algorithms provide an efficient and accurate set of optimal solutions with relatively less convergence and fast computational time. However, as PV-WT HES is rapidly growing especially for islands and remote areas, there is a need for even more accurate and highly advanced optimization approaches. Therefore, hybrid algorithms have recently been extensively applied for the sizing optimization of standalone PVWT HES. Hybrid algorithm is a combination of two or more single algorithms (modern and/or classical); this combination assumes the advantage of the complementary characteristics between the algorithms to solve complex optimization problems with different linear and non-linear constraints.

Ahmadi et al. [54] implemented the hybrid big bang-big crunch (HBB-BC) algorithm to optimize the size of a standalone hybrid PVWT-BS system in terms of minimizing the TPC of the system. ENS is used as the reliability assessment parameter in the optimization problem. The proposed HBB-BC method used PSO and mutation operator in order to prevent the trap into the local optimum value. The proposed algorithm has successfully found the optimal combination that can fully satisfy the load demand for different ENS values.

Hybrid teaching-learning-based optimization algorithm (TLBO) is a new heuristic population-based optimization with population size and number of iteration parameters. Cho et al. [38] used improved TLBO by utilizing a clonal selection optimization to find the optimal combinations for the standalone PV, WT, DG and BS system in Jeju island in South Korea with economical and reliability aspects. The economical assessment parameters used in this optimization are TAC and fuel cost while the reliability assessment parameter is LPSP. In this study, the authors used TLBO to search for the global optimal solution, and then the optimal solution is selected through the clonal selection method. Optimal sizing is achieved with a 0\% LPSP and an \$89,400 TAC.

Tito et al. [36] applied a hybrid GA and an exhaustive-search technique optimization method to size a standalone hybrid PV, WT, and battery system considering socio-demographic factors in terms of minimizing TC and ensuring system reliability to satisfy load demand. The reliability parameter used in the optimization problem is LPSP. Socio-demographic factor is used in this optimization as a social assessment parameter. The study investigated the effect of socio-demographic factors in sizing HES using the examined energy usage patterns of six different electrical users and their influence on the size of HES. The six user patterns are constructed based on 239 load profiles using Kohonen probabilistic neutral network. The authors found that the optimal size of one user at $0 \%$ LPSP cannot be exactly similar to that of other users. In this case, the generation and storage capacity should be increased to ensure that the system can meet all the load requirements of users, thereby increasing system costs.

Iterative-Pareto-Fuzzy (IPF) technique is an evolutionary algorithm that integrates the iterative, Pareto and fuzzy technologies to solve single and multi-objective optimization problems. Mukhtaruddin et al. [140] used IPF to find the optimal-mix combination of a standalone PV-WT-BS HES for Kuala Terengganu, Malaysia. EENS, D and EIR parameters are used to assess the reliability of the system while TC is used to assess the economics of the system. The optimization results provided the optimal compromised solution in minimizing TC and D while maximizing system reliability. Additionally, minimizing the unutilized excess power generated from RES yields is found to reduce TC. Abdelhak et al. [141] proposed the determination of the optimum size of hybrid PV-WT-BS system using long-term wind speed data and estimated solar irradiation. The optimum size is achieved based on the objective function of minimizing the total cost of the system. 
Zahboune et al. [35] proposed the modified electric system cascade analysis (MESCA) optimization method, which combines electric system cascade analysis (ESCA) and power pinch analyses (PoPA) to derive the optimal combination of a standalone PV, WT, and BS HES in Oujda, Morocco. The main objective function of the optimization is minimizing TAC of the system. LPSP and FEE are used in the optimization to assess the reliability of the system. The optimization process is implemented in two parts by calculating the number of PVPs, WTs, and battery units based on the hybrid cascaded table (HCT) and the allowable loss of power supply (LPSP), which checks the results of the first part by computing the obtained value of LPSP with analysis time and the difference between the desired LPSP and obtained values. The optimal design is achieved with 8 WTs, 26 PVPs, and 8 battery banks with TAC of $€$ 2391.

Dufo-Lopez et al. [21] proposed a hybrid method by combining MOEA and GA. The authors applied this hybrid method to optimize the size of a standalone hybrid PV, WT, DG, and BS system. In this study, the social parameters HDI and JC are considered for the first time in the size optimization of HES. HDI depends on the annual electrical consumption per capita, whereas JC is related to direct and indirect jobs in manufacturing, installation, and operation and maintenance of HES. The optimization process is conducted in two parts: The first part implements MOEA for component sizing in terms of minimizing NPC and maximizing HDI and JC, and the second part applies GA to optimize the control strategy in terms of NPC. The authors found that HDI can be maximized by increasing the utilization of excess energy from renewable sources to serve loads rather than dump load, hence minimizing NPC. Additionally, JC can be increased when the number of components increased in the hybrid system.

Lujano-Rojas et al. [142] proposed a hybrid MCS- and ANNbased GA optimization algorithm to find the optimal sizing for a hybrid PV-WT-DG-BS system in terms of cost and reliability for the Zaragoza area in Spain. NPC is used to assess the system economically and ENS is used to assess the system's reliability. The authors used a probabilistic method MCS to solar radiation and wind speed time series and then used generated data to train ANN-based GA. The proposed method was able to find the optimal combination of HES in a reasonable manner under conditions of uncertainty.

Katsigiannis et al. [87] proposed a hybrid simulated annealing (SA)-tabu search (TA) algorithm to optimize the size of a hybrid system for the Chania region in Greece. As SA has rapid convergence time in the neighborhood of optimal solutions and TS has high efficiency in finding the best solutions in a given neighborhood, the hybrid combination of the advantages of both algorithms yields enhanced results in dealing with the problems of having large number of diesel generator options and uncertainty in the values of many imported input parameters. The optimization objective function is to minimize the COE generated. The results showed that the proposed algorithm improved the solution quality without increasing the number of required simulations.

Markov-based GA is presented by [143] to determine the optimal size of hybrid PV-WT-DG units in terms of minimizing the TC. The environmental parameter considered in the optimization is $\mathrm{E}$ while the reliability parameter is LOLP. The authors used fuzzy-c-means (FCM) to cluster the operation states for PV, WT, and load and the Markov model to model the PV, WT, and load. Based on the models established by the Markov model, GA is then employed to find the optimal sizing for the system components. The authors concluded that the investment costs increase and fuel costs decrease with low $\mathrm{CO}_{2}$ emissions and low LOLP values.

Askarzadeh [144] proposed a discrete chaotic harmony searchbased simulated annealing algorithm (DCHSSA) as a discrete metaheuristic optimization technique that combines chaotic search
(CS), harmony search (HS), and simulated annealing (SA). The author used the proposed optimization method to find the optimal size combination for a standalone PV, WT, and BS system in terms of minimizing TAC. The reliability of the system is assessed by ensuring the energy balance between the generation and load. The proposed method has successfully found the optimal size of HES which comprised of 2 PVPs, 2 WTs, and 58 batteries.

Maleki et al. [145] proposed harmony search-based chaotic search (HSBCS) to optimize the size of a hybrid PV-WT-BS system with reverse osmosis (RO) for a remote area in Iran. The authors used ANN for the solar and wind forecasting and HSBCS for the optimal sizing of the system components. The objective function of the optimization is minimizing LCC of the system. The reliability parameter used in this optimization is LPSP. Three hybrid combinations are examined, and it was found that PV-BS with RO provides the lowest LCC at $\$ 6120$ followed by the PV-WT-BS system with RO at \$6550 LCC.

Khatib et al. [146] implemented an optimization methodology using the iterative method and GA to optimize the size of a standalone PV, WT, and BS system for Kuala Terengganu in Malaysia in terms of LPP and TC. In this study, the authors used iterative algorithm to generate a set of possible configurations of HES components, and GA to then determine the optimal configuration among the set of configurations obtained from iterative algorithm. In addition, the authors investigated the optimal tilt angle of the PV array and optimized the size of the HES inverter using iterative method. Size optimization is accomplished at different LPP values based on available daily wind speed and solar radiation.

Zhou and Sun [73] proposed an improved simulated annealing particle swarm optimization (SAPSO) algorithm to optimize the size of PV, WT, BS, and super-capacitor (SC) by minimizing the system LCC. The power balance between generation and load is used to assess the reliability of the system. The authors combined the improved SA with improved PSO to enhance the search ability and accuracy of the size optimization solutions. The proposed algorithm can find the optimal cost of the system with a total cost of \$ 5839.72 .

Ma et al. [42] adopted natural selection particle swarm optimization (NSPSO) by combining PSO with the selection strategy of GA to improve the precision of the optimal results. LCC parameter is used to assess the economics of the system while LPSP, $K_{1}$ and LEP parameters are used to assess the system's reliability. A single-objective optimization is initially performed to optimize LCC and then a multi-objective optimization to optimize the full life cycle, LPSP, LEP, and $\mathrm{K}_{1}$ of PV-WT-BS HES. The authors introduced a penalty function to realize the constants of LPSP and a weight coefficient transformation method to embody the weighting factors of LCC, LEP, and $K_{1}$.

Maleki et al. [43] used particle swarm optimization-based Monte Carlo simulation (PSOMCS) to optimize the size of a PVWT-BS HES while considering the solar and wind uncertainty calculation for all possibilities. TAC parameter is used to assess the economics of the system while power balance between generation and demand is used to assess the system's reliability. The authors implemented the optimization for three different hybrid combinations using measured solar irradiation and wind speed data. It was found that the hybrid WT-BS has the lowest TAC at $\$ 17,9472.67$ followed by the hybrid PV-WT-BS system with a TC of $\$$ $18,132.69$. However, the authors concluded that the hybrid PVWT-BS system is more reliable than the WT-BS system as it reduces the probability of having no wind generation.

Tahani et al. [147] developed hybrid flower pollination algorithm (FPA) and SA algorithm to find the optimal renewable mix with increased reliability and maximum cumulative savings. Cumulative savings is used to assess the economics of the system 


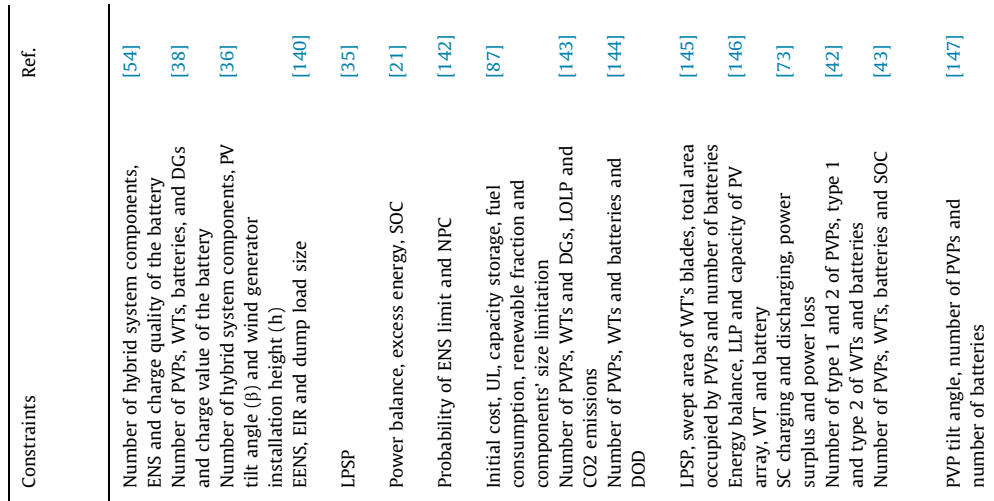

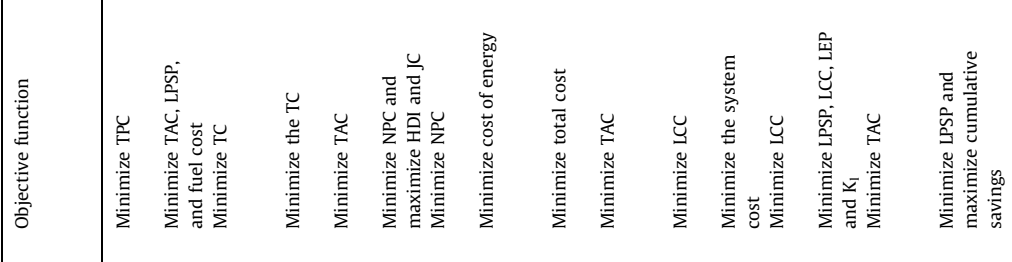

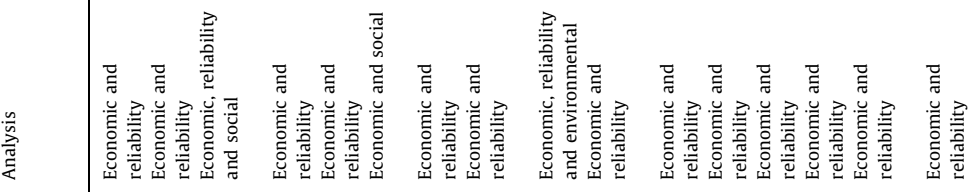

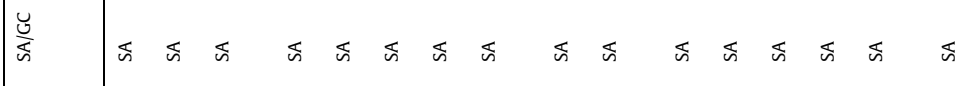

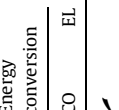

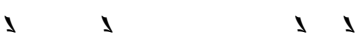

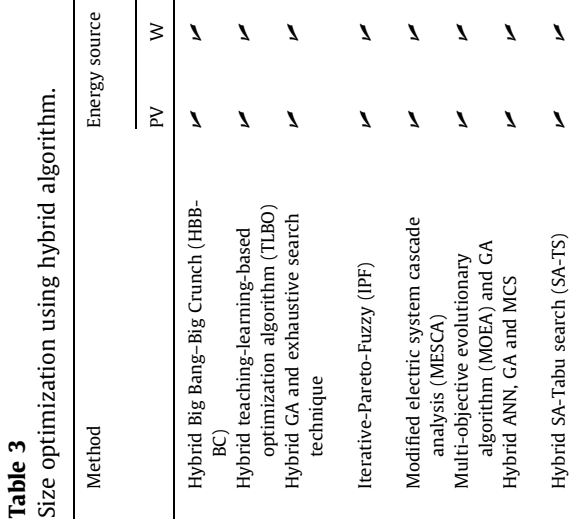

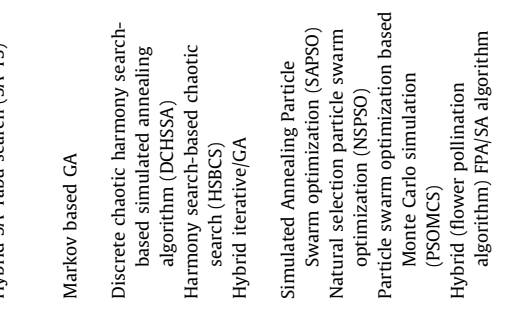




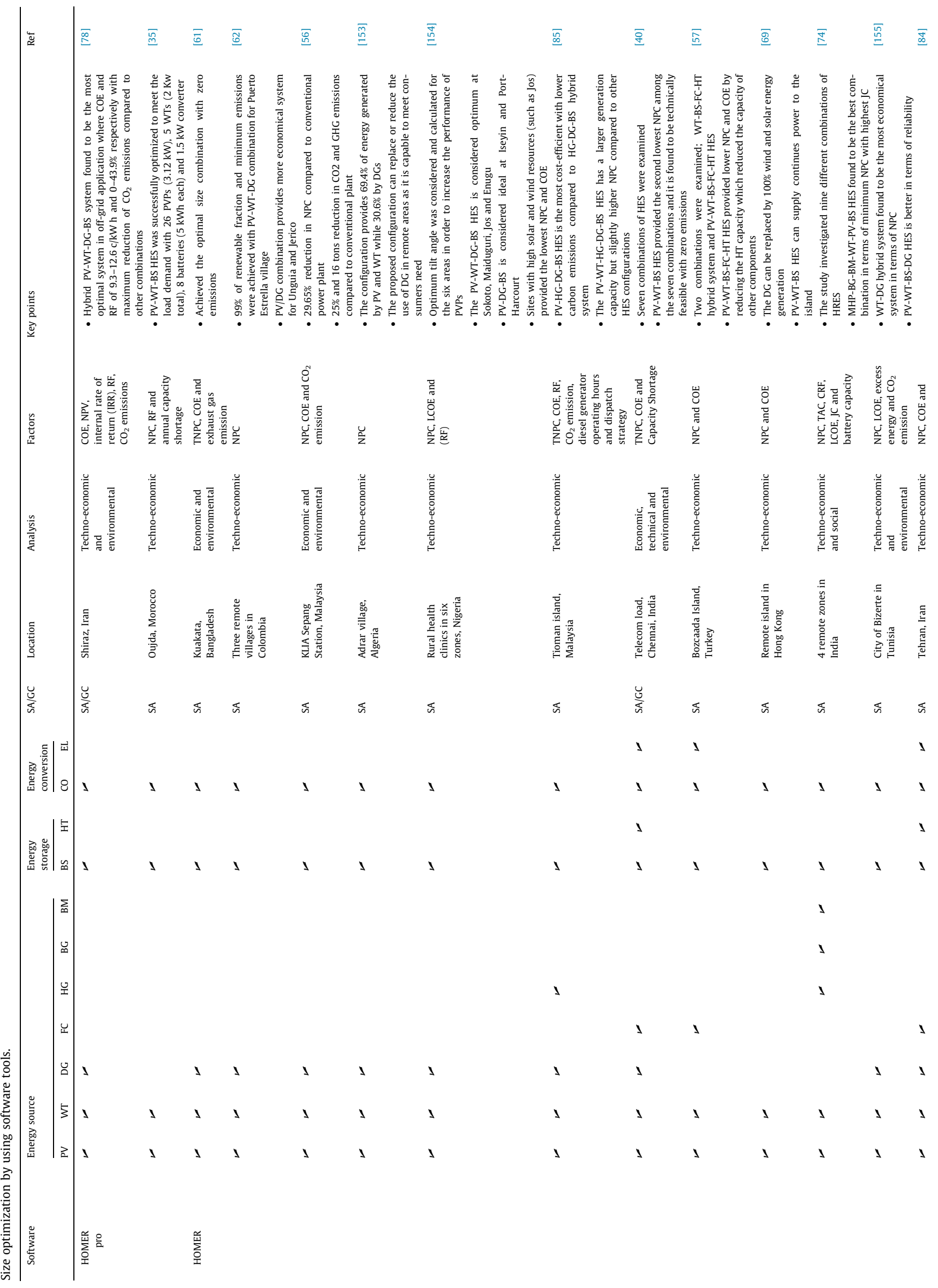




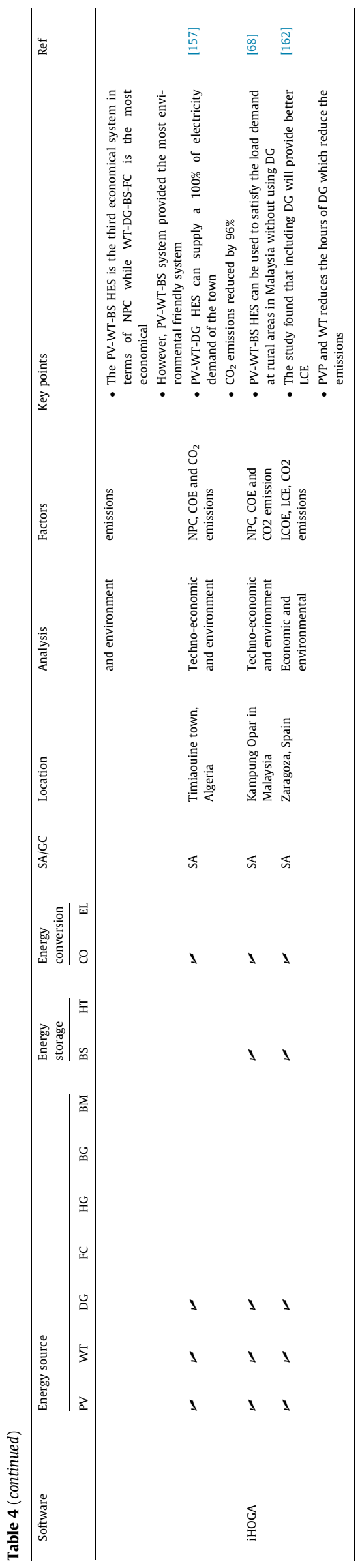

while LPSP is used to assess the system's reliability. The authors studied the optimal hybrid PV, WT, and BS system to supply electricity for a building in Tehran, Iran. In addition, the authors examined the influence of the PV panel's tilt angle on size optimization. The optimal size combination is achieved with $0 \%$ LPSP and $3.28 \%$ payback time. The summary of hybrid techniques is presented in Table 3.

\subsection{Optimization computer software tools}

Many software tools have been used in optimization of renewable energy systems [148]. Recently, two software tools, namely, HOMER and iHOGA, have been mostly used for sizing a standalone PV-WT HES.

HOMER can optimize and simulate energy systems including renewable and conventional sources, as well as an energy storage system in standalone and grid-connected modes. Optimization can be conducted using previous meteorological data according to monthly averaged or hourly data based on the site location. HOMER Pro is the improved version of HOMER with some added features such as optimizer, multi-year module, advanced battery, load profile options, monthly demand limits, and ability to link with the Matlab software [149-151]. HOMER software is widely used for sizing a standalone hybrid PV and WT energy system and other energy sources and storage systems integrated with it $[19,58,60,94,152]$. The software is limited as it performs only a single-objective optimization by minimizing NPC and the input variables should be inserted by the user [35].

These software tools are used to find the optimal design of HES for different locations worldwide by considering the main objective function of minimizing NPC and subjecting it to numerous environmental, reliability, and social constraints. Table 4 provides the selected most recent studies from different locations worldwide.

Most studies found that PV-WT-BS energy resources is more feasible as it provide the lowest NPC and COE with low or zero $\mathrm{CO}_{2}$ emissions when used in HRES [40,57]. Das et al. [61] performed a feasibility study to find the optimal size combination of HES for Kuakata, Bangladesh. The optimal size is achieved with a TNPC of $\$ 224,345$ and zero emissions. A similar study by [40] found the optimal size of HES for a telecom load in Chennai, India. Seven combinations are examined to derive the most costeffective, reliable, and less emission combination. The authors found that the PV-WT-BS system is the second most costeffective system with zero emission. Fazelpour et al. [84] reported that PV-WT-BS system is the third most economical option in terms of minimum NPC with zero emission for a building in Tehran, Iran. A techno-economic study accomplished by [69] indicated that PV-WT-BS system can completely replace diesel generator and provide an economical and reliable energy system for a remote island in Hong Kong. Zahboune et al. [35] used HOMER Pro to determine the optimal size of a PV-WT-BS system for Oujda, Morocco and compared its results with the proposed MESCA method. The optimal combination by HOMER Pro has a TNPC of $€ 27,878$.

The hybrid combination of a PV-WT-DG-BS system is widely considered for various locations. Mamaghani et al. [62] studied the optimal combinations for three different villages in Colombia. The authors found that the PV-WT-DG-BS system can provide an optimal cost-effective and reliable system with limited emissions for the Puerto Estrella village. Another study by [56] found the optimal mix of HES in terms of minimizing NPC, $\mathrm{COE}$, and $\mathrm{CO}_{2}$ emissions for the KLIA Sepang site in Malaysia. The authors compared the optimal HES with conventional plant and reported that the hybrid PV-WT-DG-BS system reduces NPC and GHG emissions compared with conventional plant. Baneshi and Hadianfard [78] carried out a techno-economic and environmental study for the 
same combination in Shiraz, Iran, wherein authors considered NPV, $\mathrm{COE}, \mathrm{RF}$, internal rate of return (IRR), and $\mathrm{CO}_{2}$ emissions in their feasibility study. The PV-WT-DG-BS system is found to be optimal in terms of the optimal $\mathrm{COE}$ and $\mathrm{RF}$ with maximum reduction of $\mathrm{CO}_{2}$ compared with other off-grid combination. Baghdadi et al. [153] conducted a techno-economic feasibility study for a PVWT-DG-BS system for Adrar, Algeria. The authors used HOMER to find the optimal combination and then conducted a power management of the system by Matlab software. The optimal combination was able to provide high RF and 70\% reduction in fossil fuel consumption. Another techno-economic study by [154] considered NPC, LCOE, and RF to determine the optimal renewable mix for six zones in Nigeria. The authors considered the tilt angle of PVP in their study to enhance PVP performance. A techno-economic, environmental study by [155] on HES for Bizerte, Tunisia reported that the PV-WT-DG-BS system is the most reliable with limited emissions, whereas the WT-DG system is the most cost-effective. A similar study by [156] found that the PV-WT-DG-BS system ensures a significant reduction in GHG and is cost-effective for a large resort in South China Sea in Malaysia. Bentouba and Bourouis [157] considered DG as a back-up source to PV and WT. The optimization results showed that PV-WT-DG HES can satisfy $100 \%$ of the load demand with a $96 \%$ reduction in carbon emissions.

A techno-economic study by [57] indicated that the PV-WT-FCBS-HT system is the most cost-effective and reliable combination for the Bozcaada island in Turkey. Moreover, the authors concluded that the increase in annual average of solar radiation and wind speed decreases both NPC and COE. Another techno-economic study is conducted by [74] to investigate the best of nine different HRES combinations. The study showed that the hybrid MHP-BGBM-WT-PV-BS system provides minimum NPC and maximum JC. A study by [85] found that HES with HG can be cost-effective and reliable with limited emissions for the Tioman Island in Malaysia.iHOGA is another software tool used in the size optimization of standalone PV-WT HES. iHOGA is a hybrid energy system optimization software developed by the electric engineering department of the University of Zaragoza [158]. The optimization can be conducted by using input data of component, economic, and constraints resources. The simulation is carried out for 1-h interval in which all system variables remain unchanged throughout the simulation. This software utilizes GA to perform the size optimization of single- or multi-objective optimization and optimal control strategies with less computational time compared to the use of GA alone. In addition, it utilizes Monte Carlo Simulation to perform probabilistic analysis [12,142]. It also can perform analysis for buy and sell for electric energy when the hybrid system is connected to the utility grid with different cases of net metering $[25,159]$ and allows for selling the surplus hydrogen produced by the electrolyzer [158]. It allows to include the MPPT function in the PV charge regulator and estimate the lifetime of the lead-acid batteries [160] based on model predication of lead-acid batteries developed by [161]. In addition, it consider the efficiency of the inverter as a function of the power output. Moreover, it considers the height of the wind turbine, and atmospheric pressure and air density in the optimization problem [158]. In version 2.0 PRO plus, the social criteria that effects the optimization of HES such as HDI and JC can be included in the optimization problem. The optimization is achieved by minimizing NPC and additional variables can be minimized such as lifecycle emissions and unmet load. Fadaeenejad et al. [68] used the iHOGA software to examine the optimal size combination for a village in Malaysia in terms of minimizing the amount of $\mathrm{CO}_{2}$ emission, NPC, and COE. The study showed that the hybrid PV-WT-BS energy system is a cost-effective and reliable option for villages in Malaysia. Dufo-Lopez et al. [162] used HOGA by utilizing two algorithms namely MOEA based on strength pareto evolutionary algorithm (SPEA) and SPEA2, and GA. MOEA algorithm is utilized to search for the optimal combinations of components in terms of minimizing costs and emissions while GA algorithm is used to find the optimal control strategy in terms with lowest cost. The authors performed four optimization cases for two locations in Spain namely Zaragoza and Jaca. The study found that the PV-WT-DG-BS system and PV-DG-BS system are the most economical and eco-friendly combination for Zaragoza and Jaca respectively.

\subsection{Comparison between algorithms}

The review demonstrates that many algorithms are applied for sizing the standalone hybrid PV-WT system coupled with different energy sources and storage systems. Table 5 summarizes the studies conducted in comparing the algorithms used in the size optimization of HES.

PSO is considered as one of the most used algorithm in HES size optimization due to its good performance, flexibility, and simplicity. However, PSO has a low performance in solving non-coordinate system as it defines the particle basis (RES components) based on three-dimensional coordinates $(\mathrm{x}, \mathrm{y}, \mathrm{z})$ only. This limitation makes the optimization results insufficient when the system consists of more than three components. Moreover, in some cases, PSO tends to converge to a local optimal solution [18]. To overcome these drawbacks, Zhou and Sun [73] proposed SAPSO. The advantages of SAPSO over conventional PSO include its ability to avoid the trapping at local optimal values and improve the diversity of PSO to solve the non-coordinate system and improve the global searching by reducing computational time. MBA is used to optimize the size of HES and is compared with PSO, CS, and ABC. This study again proved that PSO cannot provide the most optimal solution due to its low performance in non-coordinate problems. Moreover, $\mathrm{ABC}$ is compared to PSO and HOMER, which showed that $\mathrm{ABC}$ has better computational time, and better results compared with that of PSO [17]. Additionally, the study found that CS has better quality results followed by $A B C$. In the other hand, MBA can achieve the best optimal result compared to PSO, CS, and ABC with less computational time, as well as the lowest mean and standard deviation [53]. PSO can be improved in terms of computational time and convergence of results. A study by [51] proposed MPSO to find the optimal renewable mix with minimum investment cost. MPSO provided faster convergence and shorter computational time than conventional PSO.

SA is a generic probabilistic algorithm and is a good option for the global optimization problem. However, this method is not widely used in standalone HES size optimization because of the low precision of its solutions compared to other algorithms such as PSO, GA and FPA [18,41,147]. A discrete version of SA (DSA) is compared with DHS to evaluate its performance in sizing HES. Unlike DHS, DSA failed to determine the optimal solutions [138]. By taking the advantage of SA in avoiding trapping at the local minima, SA can be successfully used in HES size optimization by combining it with search algorithm. DCHSSA is a combination of SA and two search algorithms, namely, CS and HS. DCHSSA provided more precise results and is the best optimal combination of HES compared with DSA and DHSSA [144]. Another study [147] combined SA with FPA to form a hybrid FPA-SA algorithm and compared it with PSO and GA. FPA-SA is found to have better-quality results with less computational time compared to GA and precise optimal values compared to PSO.

Compared to other iterative procedures, TS is an iterative optimization method that can avoid trapping in the local solutions. However, TS needs to start from the feasible solution and requires a large number of performed simulations. In [87], the authors found that combining TS with SA overcomes this drawback. The initial feasible solution can be obtained by SA and fed to TS. There- 
Table 5

Comparison of size optimization methods.

\begin{tabular}{|c|c|c|c|c|c|}
\hline Proposed method & Compared methods & $\begin{array}{l}\text { System } \\
\text { components }\end{array}$ & Objective function & The performance of proposed method compared with other methods & Ref. \\
\hline Mine blast algorithms (MBA) & $\begin{array}{l}\text { PSO, cuckoo search }(C S) \text { and } \\
\text { artificial bee colony }(A B C)\end{array}$ & PV-WT-FC & Minimize ATC & $\begin{array}{l}\text { - MBA provides the minimal annual cost compared to the other algorithms } \\
\text { - Less computational time }\end{array}$ & [53] \\
\hline Hybrid big bang-big crunch (HBB-BC) & $\begin{array}{l}\text { PSO and discrete harmony } \\
\text { search (DHS) }\end{array}$ & PV-WT-BS & Minimize TPC & $\begin{array}{l}\text { - Higher optimal solutions accuracy } \\
\text { - Smaller standard deviation (Std.) compared to other algorithms }\end{array}$ & [54] \\
\hline Hybrid GA and exhaustive search technique & GA & PV-WT-BS & Minimize total cost & - Provides same optimal solution but with smaller number of iterations than GA & [36] \\
\hline Ant colony optimization (ACO) & $\mathrm{GA}$ and $\mathrm{ABC}$ & PV-WT-BS & Minimize total cost & $\begin{array}{l}\text { - ACO, GA and ABC provided same optimal costs } \\
\text { - ACO is faster by providing lower optimal convergence iterations and optimal } \\
\text { convergence time }\end{array}$ & [135] \\
\hline Hybrid SA-Tabu search & SA and TA & $\begin{array}{l}\text { PV-WT-BS - } \\
\text { DG-FC }\end{array}$ & Minimize COE & $\begin{array}{l}\text { - Higher quality of solutions } \\
\text { - Faster convergence }\end{array}$ & [87] \\
\hline PSO, TS and SA & $\begin{array}{l}\text { Improved PSO(IPSO), improved } \\
\text { harmony search (HIS), improved } \\
\text { harmony search-based } \\
\text { simulated annealing (IHSBSA) } \\
\text { and artificial bee swarm } \\
\text { optimization (ABSO) }\end{array}$ & PV-WT-BS & $\begin{array}{l}\text { Minimize total annual cost } \\
\text { (TAC) }\end{array}$ & $\begin{array}{l}\text { - At } \text { LPSP }_{\max }=2 \% \text {, the algorithms ranked based on mean, standard deviation, } \\
\text { worst and best indexes. The indexes have been reported over } 50 \text { runs } \\
\text { - ABSO ranked as } 1 \text { as it yields better results than the other algorithms } \\
\text { - IHSBSA, HIS, IPSO, PSO, TS and SA ranked as } 2,3,4,5,6 \text { and } 7 \text { respectively }\end{array}$ & [67] \\
\hline Artificial bee colony $(\mathrm{ABC})$ & PSO and HOMER & $\begin{array}{l}\text { PV-WT-BM- } \\
\text { BS }\end{array}$ & Minimize TC & $\begin{array}{l}\text { - ABC provided slightly better convergence rate compared to PSO by converging in } \\
\text { almost } 10 \text { th iteration } \\
\text { - ABC provided faster computational time with better quality results in terms of } \\
\text { minimizing LCOE }\end{array}$ & [17] \\
\hline Cuckoo search (CS) & PSO and GA & PV-WT-BS & Minimize total cost & $\begin{array}{l}\text { - CS faster in optimization as it reduces the computation burden } \\
\text { - CS sample the search space more efficiently and generated new solutions which } \\
\text { provided better solutions quality compared to GA and PSO }\end{array}$ & [49] \\
\hline $\begin{array}{l}\text { Natural selection particle swarm } \\
\text { optimization (NSPS) }\end{array}$ & GA & PV-WT-BS & LPSP, LCC, LEP and $K_{1}$ & $\begin{array}{l}\text { - NSPSO avoids a premature convergence effectively } \\
\text { - It provides precise results with lower fitness function value }\end{array}$ & {$[42]$} \\
\hline Enumeration-based iterative & HOMER & $\begin{array}{l}\text { PV-WT-DG- } \\
\text { BS }\end{array}$ & Minimize LCC & - The proposed algorithm provided lower LCC & [47] \\
\hline Markov based GA & Chronology-based GA & PV-WT-DG & Minimize total cost & $\begin{array}{l}\text { - Markov based GA has much smaller CPU time } \\
\text { - Markov based GA provided better cost }\end{array}$ & [143] \\
\hline Discrete harmony search (DHS) & $\begin{array}{l}\text { Discrete simulated annealing } \\
\text { (DSA) }\end{array}$ & $\begin{array}{l}\text { PV-WT-DG- } \\
\text { BS }\end{array}$ & Minimize TAC & - DHS provided better results with less run time than DSA & [138] \\
\hline $\begin{array}{l}\text { Discrete chaotic harmony search-based } \\
\text { simulated annealing algorithm (DCHSSA) }\end{array}$ & $\begin{array}{l}\text { Discrete simulated annealing } \\
\text { (DSA) and Discrete harmony } \\
\text { search-based simulated } \\
\text { annealing (DHSSA) }\end{array}$ & PV-WT-BS & Minimize TAC & $\begin{array}{l}\text { - DCHSSA yields better results in terms of mean and worst values than other } \\
\text { algorithms } \\
\text { - DCHSSA provided same ATC results as DHSSA }\end{array}$ & [144] \\
\hline $\begin{array}{l}\text { Harmony search-based chaotic search } \\
\text { (HSBCS) }\end{array}$ & Harmony search (HS) & $\begin{array}{l}\text { PV-WT-BS- } \\
\text { RO }\end{array}$ & Minimize LCC & $\begin{array}{l}\text { - It provides better average index than } \mathrm{HS} \\
\text { - It provides better standard deviation and mean simulation time than } \mathrm{HS}\end{array}$ & [145] \\
\hline $\begin{array}{l}\text { Simulated annealing particle swarm } \\
\text { optimization (SAPSO) }\end{array}$ & PSO & $\begin{array}{l}\text { PV-WT-BS- } \\
\text { SC }\end{array}$ & Minimize total cost & - SAPSO yields better cost results in less computation time & [73] \\
\hline $\begin{array}{l}\text { Modified particle awarm optimization } \\
\text { (MPSO) }\end{array}$ & PSO & PV-WT-BS & Minimize TIC & - MPSO gives fast result convergence and shorter computational time & [51] \\
\hline $\begin{array}{l}\text { Hybrid flower pollination algorithm } \\
\text { simulated annealing (FPA/SA) }\end{array}$ & GA and PSO & PV-WT-BS & $\begin{array}{l}\text { Minimize LPSP and } \\
\text { maximize cumulative } \\
\text { savings }\end{array}$ & $\begin{array}{l}\text { - FPA/SA provided better results quality than GA } \\
\text { - FPA/SA had a precise optimum values than PSO } \\
\text { - FPA/SA has better performance as it has less convergence time }\end{array}$ & [147] \\
\hline PSO & Differential evolution (DE) & $\begin{array}{l}\text { PV-WT-FC- } \\
\text { HT }\end{array}$ & Minimize TC & $\begin{array}{l}\text { - PSO has lower number of epoch } \\
\text { - PSO converging faster than DE }\end{array}$ & [75] \\
\hline $\begin{array}{l}\text { Modified electric system cascade analysis } \\
\text { (MESCA) }\end{array}$ & HOMER pro & PV-WT-BS & Minimize TAC & $\begin{array}{l}\text { - MESCA less iterations with slightly less computational time than HOMER pro } \\
\text { - MESCA provides more flexibility in selection of sources of energy types }\end{array}$ & [35] \\
\hline $\begin{array}{l}\text { Hybrid teaching-learning-based optimization } \\
\text { algorithm (TLBO) }\end{array}$ & GA and PSO & $\begin{array}{l}\text { PV-WT-BS- } \\
\text { DG }\end{array}$ & $\begin{array}{l}\text { Minimize TAC, LPSP, and } \\
\text { fuel cost }\end{array}$ & - Hybrid TLBO provided better quality results (better total cost) & [38] \\
\hline
\end{tabular}


fore, the use of such hybrid algorithm provides better-quality HES optimal size results compared to the use of TS or SA alone. In [67], the authors examined the performance of seven heuristic algorithms in terms of minimizing the TAC of different hybrid system combinations. The algorithms are ranked based on mean, standard deviation, and worst and best indexes of over 50 runs, and ABSO was found to provide better results than IHSBSA, IHS, IPSO, PSO, $\mathrm{TS}$, and SA in terms of minimizing the TAC.

GA can provide good convergence by avoiding trapping in local optimal solutions; however, it requires a large number of iterations, which increase response time [18]. To overcome this drawback, GA can be combined with an exhaustive-search method to take the advantage of GA as it can converge to a much wider search space and avoid trapping in local solution and the advantage of exhaustive-search technique to rapidly and effectively find the optimal solution within the search space [36]. Additionally, GA required long computational time as each iteration includes a set of numerous solutions. To overcome this, a stochastic model such as Markov or chronology can be used to predict the future state based on its current state only to reduce computational time and provide better results. In [143], a comparison between the Markov- and chronology-based GA in optimizing HES indicated that the former can reduce the CPU time more than the latter and provide the most feasible solution. GA has high performance in the search process, which can be combined with PSO to deal with defects of both PSO and GA by improving the precision of the results and the global optimization ability [42].

CS-based optimization methodology is efficient in finding the optimal solutions for complex problems compared to PSO and GA as it has faster computational time. Additionally, CS-based optimization methodology provides better quality results as it samples the search space more efficiently and, therefore, can be utilized to solve complex HES sizing problem and provide efficient optimal solutions [49]. ACO was compared with GA and ABC to minimize the total cost of a hybrid PV-wind battery system. The optimal cost results from all algorithms are the same. However, ACO provided the optimal solutions with less convergence iterations and faster convergence time [135].

DHS and PSO can avoid trapping at the local optimum value and continue the search for global values. However, in very complex systems, they perform with relatively high computation time and low convergence. In this context, HBB-BC can avoid trapping at local optimum values, same as DHS and PSO, and can provide faster convergence with less computational time [54].

The initial population input data of the optimization algorithms are unbounded and diverged due to random nature of renewable sources (solar radiation, wind speed, and etc.). Hence, the optimization results may be far from the minimum global solutions. In [75], the authors examined the difference between PSO and
DE algorithms in the HES size optimization. PSO provided better solutions in terms of convergence to global best values and convergence speed when compared to DE. When random initial conditions are chosen for PSO and DE algorithms, DE failed to provide global convergence as initial conditions are far away from the minimum global solution, whereas PSO yielded the optimal solutions close to minimum global solutions. Therefore, the use of PSO for HES size optimization when unbounded random initial conditions are applied is highly recommended, whereas DE can better perform when initial conditions are bounded to values near the global best value [163] and can be used along with other algorithms to compensate its population diversity decay in order to avoid suboptimal trap.

Hybrid TLBO is the combination of a search algorithm TLBO and a selection algorithm clonal selection algorithm. Hybrid TLBO uses a fewer number of parameters compared to GA and PSO but with better performance. This algorithm can provide a better-quality set of optimal solutions but might take longer computational time. However, hybrid TLBO can be considered as a good choice for HES size optimization as it has superior performance in dealing with the fluctuation of solar irradiation and wind speed data [38].

MESCA can provide almost a similar quality of optimization results compared to HOMER with less computational time as it performs the optimization with less iterations. Therefore, MESCA is recommended for HES optimization in complex sites [35]. HOMER software takes longer time to simulate HES and obtains the results compared to other artificial algorithms such as $A B C$ and PSO [17]. In another study [47], authors proposed enumeration-based iterative algorithm and compared it with HOMER to evaluate its performance in sizing HES. The study found that HOMER provided a high value of renewable energy penetration because it does not dump the excess energy generated from RES. Therefore, the proposed iterative method can yield better optimal results in terms of minimizing LCC.

\section{Critical findings and discussions}

- The implementation of HRESs provides a cost-effective and reliable option, given the fuel supply shortage and high cost associated with grid extension for islands and remote rural areas. The selection of RESs for a specific location is based on site specifications. This review shows that the most preferable hybrid energy system for islands and remote areas is the PV-WT-DGBS system as it provides reliability and ensures continuity of power supply, followed by PV-WT-BS as it is the most ecofriendly combination with zero emissions as shown in Fig. 4.

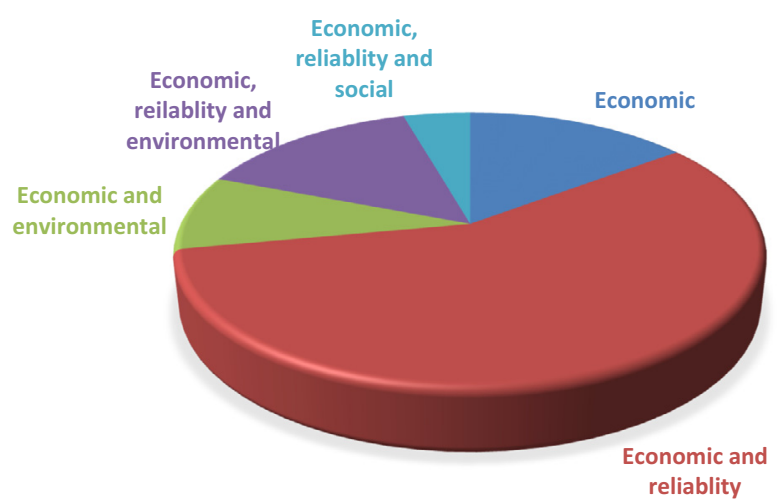

Fig. 5. Assessment parameters used for standalone PV-WT HES since 2012. 


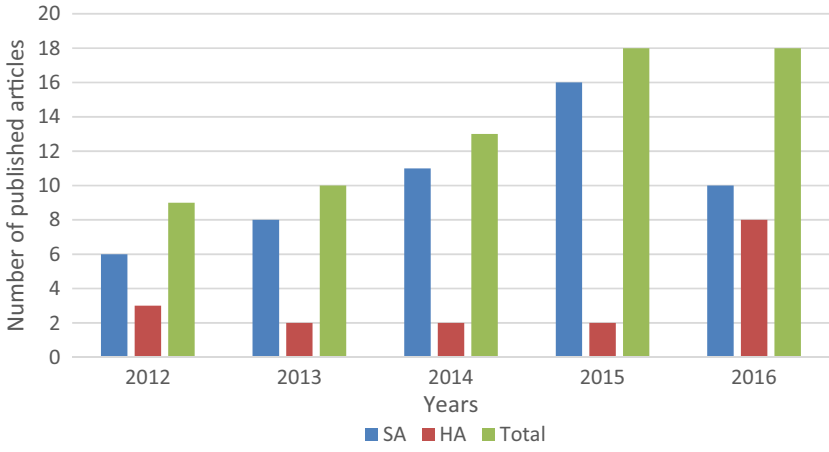

Fig. 6. Use of single and hybrid algorithms for sizing of standalone PV-WT HES from 2012 to 2016.

- As most standalone hybrid energy systems are used for remote and rural areas, in many cases, the load profile data are unavailable. Moreover, the accuracy of load profile immensely influences the size optimization results. Therefore, more research is required in the field of load profile estimation and forecasting to establish and construct more accurate predictions for the load profile. The new technique should not only focus on the variables of technical and climate specifications in forecasting process but also include social factors.

- The peaks of solar irradiation and wind speed influence the size optimization solutions. Therefore, usage of hourly annual solar and wind data rather than daily or monthly data is recommended as hourly data contain the troughs and peaks of solar irradiation and wind speed.

- Based on the reviewed studies, the manufacturing costs of hybrid system components are the main reason of the high initial costs of HES, which require a significant reduction to lessen initial system cost. This reduction will decrease payback time and increase return in investment which will eventually increase social acceptance and human development index.

- As shown in Fig. 5, few studies have considered social assessments such as human development, job creation, and social acceptance in optimization problems. These social factors are usually affected by the total cost and energy savings of HES, which influence HES sizing optimization. Therefore, considering these factors in size optimization problems is recommended.

- PV, wind, and battery system have high potential in off-grid application, thus, improvements in the life cycle of batteries and the efficiency of power converters can increase the use of this combination due to its zero-emission advantage.

- Considering the tilt angle of the PV array as a constraint in the size optimization problem is important as it affects the accuracy of the optimal results.

- The height of WT and its swept area are found be having a significant effect on the optimization results. In this context, these constraints in the optimization problem should be considered.

- From the review, it is found that most researchers look into the cost and then the reliability objective in optimization as shown in Fig. 5. Less researchers considered the environmental objective function. Cost and reliability are the criteria given the most concern in the hybrid system. However, environmental objective especially when the hybrid system contains conventional energy source should be given increased attention.

- As can be noticed from this review, the use of classical size optimization methods recently declined, and a growing trend is observed toward the use of modern size optimization methods as they provide a set of optimal results that allow decisionmakers to select the best suitable combination of HES. Therefore, the use of modern methods in HES is recommended as they can provide promising and realistic optimal sizes.
- Owing to the stochastic nature and the capability of artificial algorithms in solving multi-objective, non-linear, and complex optimization problems, these methods have attracted much attention as the usage of artificial methods in HES size optimization drew more attention than the classical methods.

- SA algorithm is not widely used in HES size optimization. However, SA can escape from trapping at local solutions. Therefore, SA is best used when it is combined with other evolutionary algorithms and/or search algorithms to improve the accuracy of its optimal solutions and enhance the global search.

- PSO has been widely used in sizing HES. However, conventional PSO suffers from premature convergence. Therefore, in current studies, the use of conventional PSO alone in size optimization of HES started to decline. As a result, the use of PSO variant and improved versions of PSO is currently utilized by many studies as these improved versions provide better results compared to conventional PSO.

- As the hybrid energy system optimization considered a complex problem, many objective functions and constraints should be considered to improve quality results. Numerous studies currently use triple-objective optimization problems as these provide more realistic solutions and, hence, more accurate optimal results.

- The most common optimization method implemented for triple-objective problems is NSGA-II. NSGA-II and its variant (such as controlled elitist genetic algorithm) provided good performance in solving triple-objective optimization problem. Additionally, PICEA demonstrated good performance in solving multi-objective functions. Therefore, the use of this algorithm in HES size optimization problem is recommended.

- Single algorithms using artificial methods provide good performance in solving size optimization problem of HES, whereas hybrid algorithms perform better with more promising results. In this context, the use of hybrid algorithms recently extensively increased in size optimization for standalone PV-WT HES as shown in Fig. 6.

- As can be seen from this review, newly developed hybrid algorithms such as TLBO, FPA/SA, and NSPSO provide better-quality results with less computational time compared with GA and PSO.

\section{Conclusion}

This paper presents a comprehensive review and critical comparison of most recent size optimization methods of standalone solar and wind based hybrid energy systems. The most popular hybrid combination for islands and remote rural areas found to be the solar, wind, diesel generator, and battery storage based hybrid energy system as it provides more reliable and continuous power supply. Finding the optimum size of each element is a key factor to reduce the cost while maintaining the reliability and social acceptance.

In order to solve a sizing optimization problem of a standalone solar and wind hybrid energy system, various assessment parameters such as economical, reliability, environmental and social parameters are explained and summarized. The selection of some of these parameters is essentially to obtain an optimal combination for the standalone solar and wind system. Moreover, the metrological data and load profile have an impact on the size optimization problem. Based on the review, it is observed that the use of forecasted solar, wind, and load profile data in the optimization problem improve the size optimization results compared to the use of historical data.

Most of the papers for sizing a standalone solar and wind hybrid system are carried out based on single algorithms including classical and artificial methods. Artificial methods using single algo- 
rithms has the ability to search for local and global optima and provide a set of optimal results with less computational time. Therefore, artificial methods have attracted much attention in sizing of standalone solar and wind system than classical methods. However, as a standalone solar and wind systems are rapidly growing especially for islands and remote areas, there is a need for even much accurate and highly advanced optimization approaches. Therefore, hybrid algorithms have recently been extensively applied for the sizing optimization of standalone solar and wind hybrid system. Moreover, software computer tools are also used widely for sizing and designing of standalone solar and wind system. However, using modern methods such as artificial algorithms and hybrid algorithms provide more accurate optimization results than software tools as they have the ability to solve multi-objective optimization problems.

\section{References}

[1] Initiative GE. Global electricity initiative executive summary 2014. GEI2014.

[2] Kuang Y, Zhang Y, Zhou B, Li C, Cao Y, Li L, et al. A review of renewable energy utilization in islands. Renew Sustain Energy Rev 2016;59:504-13.

[3] Sinha S, Chandel SS. Review of recent trends in optimization techniques for solar photovoltaic-wind based hybrid energy systems. Renew Sustain Energy Rev 2015;50:755-69.

[4] Van TV, Norton M, Ivanov C, Delimar M, Hatziargyriou N, Stromsather J, et al. Organic growth: toward a holistic approach to European research and innovation. IEEE Power Energy Mag 2015;13:30-7.

[5] "Monitoring report 2015". Bundesnetzagentur für Elektrizität, Gas, Telekommunikation, Post und Eisenbahnen; 2016.

[6] Kroposki B, Mather B. Rise of distributed power integrating solar energy into the grid. IEEE Power Energy Mag 2015;13:14-8.

[7] Cheng M, Zhu Y. The state of the art of wind energy conversion systems and technologies: a review. Energy Convers Manage 2014;88:332-47.

[8] Khare V, Nema S, Baredar P. Solar-wind hybrid renewable energy system: a review. Renew Sustain Energy Rev 2016;58:23-33.

[9] Zhang D, Evangelisti S, Lettieri P, Papageorgiou LG. Economic and environmental scheduling of smart homes with microgrid: DER operation and electrical tasks. Energy Convers Manage 2016;110:113-24.

[10] Koohi-Kamali S, Rahim NA. Coordinated control of smart microgrid during and after islanding operation to prevent under frequency load shedding using energy storage system. Energy Convers Manage 2016;127:623-46.

[11] Camblong H, Baudoin S, Vechiu I, Etxeberria A. Design of a SOFC/GT/SCS hybrid power system to supply a rural isolated microgrid. Energy Convers Manage 2016;117:12-20.

[12] Chauhan A, Saini RP. A review on Integrated Renewable Energy System based power generation for stand-alone applications: Configurations, storage options, sizing methodologies and control. Renew Sustain Energy Rev 2014;38:99-120.

[13] Mannke F, Mohee R, Schulte V, Surroop D. Climate-smart technologiesintegrating renewable energy and energy efficiency in mitigation and adaptation responses. Berlin/Heidelberg; 2013.

[14] Blechinger P, Cader C, Bertheau P, Huyskens H, Seguin R, Breyer C. Global analysis of the techno-economic potential of renewable energy hybrid systems on small islands. Energy Policy 2016;98:674-87.

[15] Shirley R, Kammen D. Renewable energy sector development in the Caribbean: Current trends and lessons from history. Energy Policy 2013;57:244-52.

[16] Petrakopoulou F. On the economics of stand-alone renewable hybrid power plants in remote regions. Energy Convers Manage 2016;118:63-74.

[17] Singh S, Singh M, Kaushik SC. Feasibility study of an islanded microgrid in rural area consisting of PV, wind, biomass and battery energy storage system. Energy Convers Manage 2016;128:178-90.

[18] Zahraee SM, Khalaji Assadi M, Saidur R. Application of artificial intelligence methods for hybrid energy system optimization. Renew Sustain Energy Rev 2016;66:617-30.

[19] Ramli MAM, Hiendro A, Al-Turki YA. Techno-economic energy analysis of wind/solar hybrid system: Case study for western coastal area of Saudi Arabia. Renewable Energy 2016;91:374-85.

[20] Siddaiah R, Saini RP. A review on planning, configurations, modeling and optimization techniques of hybrid renewable energy systems for off grid applications. Renew Sustain Energy Rev 2016;58:376-96.

[21] Dufo-López R, Cristóbal-Monreal IR, Yusta JM. Optimisation of PV-winddiesel-battery stand-alone systems to minimise cost and maximise human development index and job creation. Renewable Energy 2016;94:280-93.

[22] Ribeiro AED, Arouca MC, Coelho DM. Electric energy generation from smallscale solar and wind power in Brazil: The influence of location, area and shape. Renewable Energy 2016;85:554-63.

[23] Mahesh A, Sandhu KS. Hybrid wind/photovoltaic energy system developments: Critical review and findings. Renew Sustain Energy Rev 2015;52:1135-47.
[24] Al Busaidi AS, Kazem HA, Al-Badi AH, Farooq Khan M. A review of optimum sizing of hybrid PV-Wind renewable energy systems in oman. Renew Sustain Energy Rev 2016;53:185-93.

[25] Upadhyay S, Sharma MP. A review on configurations, control and sizing methodologies of hybrid energy systems. Renew Sustain Energy Rev 2014;38:47-63.

[26] Olatomiwa L, Mekhilef S, Huda ASN, Ohunakin OS. Economic evaluation of hybrid energy systems for rural electrification in six geo-political zones of Nigeria. Renewable Energy 2015;83:435-46.

[27] Zhou W, Lou C, Li Z, Lu L, Yang H. Current status of research on optimum sizing of stand-alone hybrid solar-wind power generation systems. App Energy 2010;87:380-9.

[28] Fathima AH, Palanisamy K. Optimization in microgrids with hybrid energy systems - A review. Renew Sustain Energy Rev 2015;45:431-46.

[29] Shivarama Krishna K, Sathish Kumar K. A review on hybrid renewable energy systems. Renew Sustain Energy Rev 2015;52:907-16.

[30] Askarzadeh A. Optimisation of solar and wind energy systems: a survey. Int J Ambient Energy 2016:1-10.

[31] Erdinc O, Uzunoglu M. Optimum design of hybrid renewable energy systems: Overview of different approaches. Renew Sustain Energy Rev 2012;16:1412-25.

[32] Fadaee M, Radzi MAM. Multi-objective optimization of a stand-alone hybrid renewable energy system by using evolutionary algorithms: A review. Renew Sustain Energy Rev 2012;16:3364-9.

[33] Luna-Rubio R, Trejo-Perea M, Vargas-Vázquez D, Ríos-Moreno GJ. Optimal sizing of renewable hybrids energy systems: A review of methodologies. Sol Energy 2012;86:1077-88.

[34] Bourennani F, Rahnamayan S, Naterer GF. Optimal design methods for hybrid renewable energy systems. Int J Green Energy 2014;12:148-59.

[35] Zahboune H, Zouggar S, Krajacic G, Varbanov PS, Elhafyani M, Ziani E. Optimal hybrid renewable energy design in autonomous system using Modified Electric System Cascade Analysis and Homer software. Energy Convers Manage 2016;126:909-22.

[36] Tito SR, Lie TT, Anderson TN. Optimal sizing of a wind-photovoltaic-battery hybrid renewable energy system considering socio-demographic factors. Sol Energy 2016;136:525-32.

[37] Kamjoo A, Maheri A, Dizqah AM, Putrus GA. Multi-objective design under uncertainties of hybrid renewable energy system using NSGA-II and chance constrained programming. Int J Electr Power Energy Syst 2016;74:187-94.

[38] Cho J-H, Chun M-G, Hong W-P. Structure optimization of stand-alone renewable power systems based on multi object function. Energies 2016;9:649.

[39] Baghaee HR, Mirsalim M, Gharehpetian GB, Talebi HA. Reliability/cost-based multi-objective Pareto optimal design of stand-alone wind/PV/FC generation microgrid system. Energy 2016;115:1022-41.

[40] Margaret Amutha W, Rajini V. Techno-economic evaluation of various hybrid power systems for rural telecom. Renew Sustain Energy Rev 2015;43:553-61.

[41] Maleki A, Ameri M, Keynia F. Scrutiny of multifarious particle swarm optimization for finding the optimal size of a PV/wind/battery hybrid system. Renewable Energy 2015;80:552-63.

[42] Ma G, Xu G, Chen Y, Ju R. Multi-objective optimal configuration method for a standalone wind-solar-battery hybrid power system. IET Renew Power Gener 2016.

[43] Maleki A, Khajeh MG, Ameri M. Optimal sizing of a grid independent hybrid renewable energy system incorporating resource uncertainty, and load uncertainty. Int J Electr Power Energy Syst 2016;83:514-24.

[44] Nehrir MH, Wang C, Strunz K, Aki H, Ramakumar R, Bing J, et al. A review of hybrid renewable/alternative energy systems for electric power generation: configurations, control, and applications. IEEE Trans Sustain Energy 2011;2:392-403.

[45] Malheiro A, Castro PM, Lima RM, Estanqueiro A. Integrated sizing and scheduling of wind/PV/diesel/battery isolated systems. Renewable Energy 2015;83:646-57.

[46] Gan LK, Shek JKH, Mueller MA. Hybrid wind-photovoltaic-diesel-battery system sizing tool development using empirical approach, life-cycle cost and performance analysis: A case study in Scotland. Energy Convers Manage 2015;106:479-94.

[47] Bhuiyan FA, Yazdani A, Primak SL. Optimal sizing approach for islanded microgrids. IET Renew Power Gener 2015;9:166-75.

[48] Zhao B, Zhang X, Li P, Wang K, Xue M, Wang C. Optimal sizing, operating strategy and operational experience of a stand-alone microgrid on Dongfushan Island. Appl Energy 2014;113:1656-66.

[49] Sanajaoba S, Fernandez E. Maiden application of Cuckoo Search algorithm for optimal sizing of a remote hybrid renewable energy System. Renewable Energy 2016;96:1-10.

[50] Hosseinalizadeh R, Shakouri H, Mohsen G, Amalnick S, Taghipour P. Economic sizing of a hybrid (PV-WT-FC) renewable energy system (HRES) for standalone usages by an optimization-simulation model: Case study of Iran. Renew Sustain Energy Rev 2016;54:139-50.

[51] Hassan A, Kandil M, Saadawi M, Saeed M. Modified particle swarm optimisation technique for optimal design of small renewable energy system supplying a specific load at Mansoura University. IET Renew Power Gener 2015;9:474-83.

[52] Gharavi H, Ardehali MM, Ghanbari-Tichi S. Imperial competitive algorithm optimization of fuzzy multi-objective design of a hybrid green power system 
with considerations for economics, reliability, and environmental emissions. Renewable Energy 2015;78:427-37.

[53] Fathy A. A reliable methodology based on mine blast optimization algorithm for optimal sizing of hybrid PV-wind-FC system for remote area in Egypt. Renewable Energy 2016;95:367-80.

[54] Ahmadi S, Abdi S. Application of the Hybrid Big Bang-Big Crunch algorithm for optimal sizing of a stand-alone hybrid PV/wind/battery system. Sol Energy 2016;134:366-74.

[55] Ogunjuyigbe ASO, Ayodele TR, Akinola OA. Optimal allocation and sizing of PV/Wind/Split-diesel/Battery hybrid energy system for minimizing life cycle cost, carbon emission and dump energy of remote residential building. Appl Energy 2016;171:153-71.

[56] Shezan SA, Julai S, Kibria MA, Ullah KR, Saidur R, Chong WT, et al Performance analysis of an off-grid wind-PV (photovoltaic)-diesel-battery hybrid energy system feasible for remote areas. J Clean Prod 2016:125:121-32.

[57] Kalinci Y, Hepbasli A, Dincer I. Techno-economic analysis of a stand-alone hybrid renewable energy system with hydrogen production and storage options. Int J Hydrogen Energy 2015;40:7652-64.

[58] Adaramola MS, Agelin-Chaab M, Paul SS. Analysis of hybrid energy systems for application in southern Ghana. Energy Convers Manage 2014;88: 284-95.

[59] Celik AN. Optimisation and techno-economic analysis of autonomous photovoltaic-wind hybrid energy systems in comparison to single photovoltaic and wind systems. Energy Convers Manage 2002;43:2453-68.

[60] Bhattacharjee S, Acharya S. PV-wind hybrid power option for a low wind topography. Energy Convers Manage 2015;89:942-54.

[61] Das HS, Dey A, Wei TC, Yatim AHM. Feasibility analysis of standalone PV/ wind/battery hybrid energy system for Rural Bangladesh. Int J Renew Energy Res 2016;6:403-12.

[62] Haghighat Mamaghani A, Avella Escandon SA, Najafi B, Shirazi A, Rinaldi F Techno-economic feasibility of photovoltaic, wind, diesel and hybrid electrification systems for off-grid rural electrification in Colombia. Renewable Energy 2016;97:293-305.

[63] Nandi SK, Ghosh HR. Prospect of wind-PV-battery hybrid power system as an alternative to grid extension in Bangladesh. Energy 2010;35:3040-7.

[64] Ahadi A, Kang S-K, Lee J-H. A novel approach for optimal combinations of wind, PV, and energy storage system in diesel-free isolated communities. Appl Energy 2016;170:101-15.

[65] Derakhshan G, Shayanfar HA, Kazemi A. Optimal design of solar PV-WT-SB based smart microgrid using NSHCSO. Int J Hydrogen Energy 2016.

[66] Askarzadeh A, dos Santos Coelho L. A novel framework for optimization of a grid independent hybrid renewable energy system: A case study of Iran. Sol Energy 2015;112:383-96.

[67] Maleki A, Pourfayaz F. Optimal sizing of autonomous hybrid photovoltaic/ wind/battery power system with LPSP technology by using evolutionary algorithms. Sol Energy 2015;115:471-83.

[68] Fadaeenejad M, Radzi MAM, AbKadir MZA, Hizam H. Assessment of hybrid renewable power sources for rural electrification in Malaysia. Renew Sustain Energy Rev 2014;30:299-305.

[69] Ma T, Yang H, Lu L. A feasibility study of a stand-alone hybrid solar-windbattery system for a remote island. Appl Energy 2014;121:149-58.

[70] Maleki A, Askarzadeh A. Comparative study of artificial intelligence techniques for sizing of a hydrogen-based stand-alone photovoltaic/wind hybrid system. Int J Hydrogen Energy 2014;39:9973-84.

[71] Maleki A, Askarzadeh A. Artificial bee swarm optimization for optimum sizing of a stand-alone PV/WT/FC hybrid system considering LPSP concept. So Energy 2014;107:227-35.

[72] Ma T, Yang H, Lu L, Peng J. Technical feasibility study on a standalone hybrid solar-wind system with pumped hydro storage for a remote island in Hong Kong. Renewable Energy 2014;69:7-15.

[73] Zhou T, Sun W. Optimization of battery-supercapacitor hybrid energy storage station in wind/solar generation system. IEEE Trans Sustain Energy 2014;5:408-15.

[74] Chauhan A, Saini RP. Techno-economic feasibility study on Integrated Renewable Energy System for an isolated community of India. Renew Sustain Energy Rev 2016;59:388-405.

[75] Sanchez VM, Chavez-Ramirez AU, Duron-Torres SM, Hernandez J, Arriaga LG, Ramirez JM. Techno-economical optimization based on swarm intelligence algorithm for a stand-alone wind-photovoltaic-hydrogen power system at south-east region of Mexico. Int J Hydrogen Energy 2014;39:16646-55.

[76] Smaoui M, Abdelkafi A, Krichen L. Optimal sizing of stand-alone photovoltaic/ wind/hydrogen hybrid system supplying a desalination unit. Sol Energy 2015;120:263-76.

[77] Rajanna S, Saini RP. Development of optimal integrated renewable energy model with battery storage for a remote Indian area. Energy 2016;111:803-17.

[78] Baneshi M, Hadianfard F. Techno-economic feasibility of hybrid diesel/PV wind/battery electricity generation systems for non-residential large electricity consumers under southern Iran climate conditions. Energy Convers Manage 2016;127:233-44.

[79] Belfkira R, Zhang L, Barakat G. Optimal sizing study of hybrid wind/PV/diese power generation unit. Sol Energy 2011;85:100-10.

[80] Shi B, Wu W, Yan L. Size optimization of stand-alone PV/wind/diesel hybrid power generation systems. J Taiwan Instit Chem Eng 2016.
[81] Suhane P, Rangnekar S, Khare A, Mittal A. Sizing and performance analysis of standalone wind-photovoltaic based hybrid energy system using ant colony optimisation. IET Renew Power Gener 2016;10:964-72.

[82] Zhao J, Yuan X. Multi-objective optimization of stand-alone hybrid PV-winddiesel-battery system using improved fruit fly optimization algorithm. Soft Comput 2015;20:2841-53.

[83] Shi Z, Wang R, Zhang T. Multi-objective optimal design of hybrid renewable energy systems using preference-inspired coevolutionary approach. Sol Energy 2015;118:96-106.

[84] Fazelpour F, Soltani N, Rosen MA. Economic analysis of standalone hybrid energy systems for application in Tehran, Iran. Int J Hydrogen Energy 2016;41:7732-43.

[85] Khan MRB, Jidin R, Pasupuleti J, Shaaya SA. Optimal combination of solar, wind, micro-hydro and diesel systems based on actual seasonal load profiles for a resort island in the South China Sea. Energy 2015;82:80-97.

[86] Bekele G, Tadesse G. Feasibility study of small Hydro/PV/Wind hybrid system for off-grid rural electrification in Ethiopia. Appl Energy 2012;97: 5-15.

[87] Katsigiannis YA, Georgilakis PS, Karapidakis ES. Hybrid Simulated AnnealingTabu Search Method For Optimal Sizing Of Autonomous Power Systems With Renewables. IEEE Trans Sustain Energy 2012;3.

[88] Chmiel Z, Bhattacharyya SC. Analysis of off-grid electricity system at Isle of Eigg (Scotland): Lessons for developing countries. Renewable Energy 2015;81:578-88.

[89] Sen R, Bhattacharyya SC. Off-grid electricity generation with renewable energy technologies in India: An application of HOMER. Renewable Energy 2014;62:388-98.

[90] Gupta RA, Kumar R, Bansal AK. BBO-based small autonomous hybrid power system optimization incorporating wind speed and solar radiation forecasting. Renew Sustain Energy Rev 2015;41:1366-75.

[91] Khatib T, Ibrahim IA, Mohamed A. A review on sizing methodologies of photovoltaic array and storage battery in a standalone photovoltaic system. Energy Convers Manage 2016;120:430-48.

[92] Louie $H$. Operational analysis of hybrid solar/wind microgrids using measured data. Energy Sustain Dev 2016;31:108-17.

[93] Hocaoglu FO, Gerek ON, Kurban M. The effect of model generated solar radiation data usage in hybrid (wind-PV) sizing studies. Energy Convers Manage 2009:50:2956-63.

[94] Sinha S, Chandel SS. Prospects of solar photovoltaic-micro-wind based hybrid power systems in western Himalayan state of Himachal Pradesh in India. Energy Convers Manage 2015;105:1340-51.

[95] Rajkumar RK, Ramachandaramurthy VK, Yong BL, Chia DB. Technoeconomical optimization of hybrid pv/wind/battery system using NeuroFuzzy. Energy 2011;36:5148-53.

[96] Nogueira CEC, Vidotto ML, Niedzialkoski RK, de Souza SNM, Chaves LI, Edwiges T, et al. Sizing and simulation of a photovoltaic-wind energy system using batteries, applied for a small rural property located in the south of Brazil. Renew Sustain Energy Rev 2014;29:151-7.

[97] Khatod DK, Pant V, Sharma J. Analytical approach for well-being assessment of small autonomous power systems with solar and wind energy sources. IEEE Trans Energy Convers 2010;25:535-45.

[98] Ekren O, Ekren BY. Size optimization of a PV/wind hybrid energy conversion system with battery storage using simulated annealing. Appl Energy 2010;87:592-8.

[99] Azimi R, Ghayekhloo M, Ghofrani M. A hybrid method based on a new clustering technique and multilayer perceptron neural networks for hourly solar radiation forecasting. Energy Convers Manage 2016;118:331-44.

[100] Chen H-C. Optimum capacity determination of stand-alone hybrid generation system considering cost and reliability. Appl Energy 2013;103:155-64.

[101] Vasilj J, Sarajcev P, Jakus D. Estimating future balancing power requirements in wind-PV power system. Renewable Energy 2016;99:369-78.

[102] Santana ÁL, Conde GB, Rego LP, Rocha CA, Cardoso DL, Costa JCW, et al. PREDICT - Decision support system for load forecasting and inference: A new undertaking for Brazilian power suppliers. Int J Electr Power Energy Syst 2012;38:33-45

[103] Borunda M, Jaramillo OA, Reyes A, Ibargüengoytia PH. Bayesian networks in renewable energy systems: A bibliographical survey. Renew Sustain Energy Rev 2016;62:32-45.

[104] Lü X, Lu T, Kibert CJ, Viljanen M. Modeling and forecasting energy consumption for heterogeneous buildings using a physical-statistical approach. Appl Energy 2015;144:261-75.

[105] Ghayekhloo M, Menhaj MB, Ghofrani M. A hybrid short-term load forecasting with a new data preprocessing framework. Electric Power Syst Res 2015;119:138-48.

[106] Al-Falahi MD, Wanik MZC. Modeling and performance analysis of hybrid power system for residential application. In: Power Engineering Conference (AUPEC), 2015 Australasian Universities; 2015. p. 1-6.

[107] Shrivastava V, Misra RB. Development of Bayesian belief network model for electrical load demand. Int J Syst Assur Eng Manage 2010;1:170-7.

[108] Panapakidis IP. Clustering based day-ahead and hour-ahead bus load forecasting models. Int J Electr Power Energy Syst 2016;80:171-8.

[109] Al-Falahi MD, Nimma KS, Jayasinghe S, Enshaei H. Sizing and modeling of a standalone hybrid renewable energy system. In: Power Electronics Conference (SPEC), IEEE Annual Southern; 2016. p. 1-6.

[110] Pillai GG, Putrus GA, Pearsall NM. Generation of synthetic benchmark electrical load profiles using publicly available load and weather data. Int ] Electr Power Energy Syst 2014;61:1-10. 
[111] Guarino F, Cassarà P, Longo S, Cellura M, Ferro E. Load match optimisation of a residential building case study: A cross-entropy based electricity storage sizing algorithm. Appl Energy 2015;154:380-91.

[112] Bahramara S, Moghaddam MP, Haghifam MR. Optimal planning of hybrid renewable energy systems using HOMER: A review. Renew Sustain Energy Rev 2016;62:609-20.

[113] Sharafi M, Elmekkawy TY. Multi-objective optimal design of hybrid renewable energy systems using PSO-simulation based approach. Renewable Energy 2014;68:67-79.

[114] El Alimi S, Maatallah T, Ben Nasrallah S. Break-even analysis and optimization of a stand-alone hybrid system with battery storage for residential load consumption-A case study. Renew Sustain Energy Rev 2014;37:408-23.

[115] Belmili H, Haddadi M, Bacha S, Almi MF, Bendib B. Sizing stand-alone photovoltaic-wind hybrid system: Techno-economic analysis and optimization. Renew Sustain Energy Rev 2014;30:821-32.

[116] Xu L, Ruan X, Mao C, Zhang B, Luo Y. An improved optimal sizing method for wind-solar-battery hybrid power system. IEEE Trans Sustain Energy 2013;4:774-85.

[117] Ma T, Yang H, Lu L, Peng J. Optimal design of an autonomous solar-windpumped storage power supply system. Appl Energy 2015;160:728-36.

[118] Kaabeche A, Ibtiouen R. Techno-economic optimization of hybrid photovoltaic/wind/diesel/battery generation in a stand-alone power system. Sol Energy 2014;103:171-82.

[119] Kaldellis JK, Zafirakis D, Kavadias K. Minimum cost solution of windphotovoltaic based stand-alone power systems for remote consumers. Energy Policy 2012;42:105-17.

[120] Khatib T, Mohamed A, Sopian K, Mahmoud M. Optimal sizing of hybrid pv/ wind systems for Malaysia using loss of load probability. Energy Sour, Part A: Recovery, Utiliz, Environ Effects 2015;37:687-95.

[121] Mokheimer EMA, Sahin AZ, Al-Sharafi A, Ali AI. Modeling and optimization of hybrid wind-solar-powered reverse osmosis water desalination system in Saudi Arabia. Energy Convers Manage 2013;75:86-97.

[122] Huneke F, Henkel J, González JAB, Erdmann G. Optimisation of hybrid off-grid energy systems by linear programming. Energy Sustain Soc 2012.

[123] Ferrer-Martí L, Domenech B, García-Villoria A, Pastor R. A MILP model to design hybrid wind-photovoltaic isolated rural electrification projects in developing countries. Eur J Oper Res 2013;226:293-300.

[124] Tan W-S, Hassan MY, Majid MS, Abdul Rahman H. Optimal distributed renewable generation planning: A review of different approaches. Renew Sustain Energy Rev 2013;18:626-45.

[125] Lee SC. Numerical estimation model of energy conversion for small hybrid solar-wind system. Sol Energy 2012;86:3125-36.

[126] Kanase-Patil AB, Saini RP, Sharma MP. Sizing of integrated renewable energy system based on load profiles and reliability index for the state of Uttarakhand in India. Renewable Energy 2011;36:2809-21.

[127] Elliston B, MacGill I, Diesendorf M. Least cost $100 \%$ renewable electricity scenarios in the Australian National Electricity Market. Energy Policy 2013;59:270-82.

[128] Gan LK, Shek JKH, Mueller MA. Optimised operation of an off-grid hybrid wind-diesel-battery system using genetic algorithm. Energy Convers Manage 2016;126:446-62.

[129] Merei G, Berger C, Sauer DU. Optimization of an off-grid hybrid PV-WindDiesel system with different battery technologies using genetic algorithm. Sol Energy 2013;97:460-73.

[130] Sheng W, Liu K-Y, Meng X, Ye X, Liu Y. Research and practice on typical modes and optimal allocation method for PV-Wind-ES in Microgrid. Electr Power Syst Res 2015;120:242-55.

[131] Abbes D, Martinez A, Champenois G. Life cycle cost, embodied energy and loss of power supply probability for the optimal design of hybrid power systems. Math Comput Simul 2014;98:46-62.

[132] Paliwal P, Patidar NP, Nema RK. Determination of reliability constrained optimal resource mix for an autonomous hybrid power system using Particle Swarm Optimization. Renewable Energy 2014;63:194-204.

[133] Borhanazad H, Mekhilef S, Gounder Ganapathy V, Modiri-Delshad M, Mirtaheri A. Optimization of micro-grid system using MOPSO. Renewable Energy 2014;71:295-306.

[134] Safari S, Ardehali MM, Sirizi MJ. Particle swarm optimization based fuzzy logic controller for autonomous green power energy system with hydrogen storage. Energy Convers Manage 2013;65:41-9.

[135] Fetanat A, Khorasaninejad E. Size optimization for hybrid photovoltaic-wind energy system using ant colony optimization for continuous domains based integer programming. Appl Soft Comput 2015;31:196-209.

[136] Wang R, Purshouse RC, Fleming PJ. Preference-inspired coevolutionary algorithms for many-objective optimization. IEEE Trans Evol Comput 2013:17:474-94.

[137] Kumar R, Gupta RA, Bansal AK. Economic analysis and power management of a stand-alone wind/photovoltaic hybrid energy system using biogeography based optimization algorithm. Swarm Evolution Comput 2013;8:33-43.

[138] Maleki A, Askarzadeh A. Optimal sizing of a PV/wind/diesel system with battery storage for electrification to an off-grid remote region: A case study of Rafsanjan, Iran. Sustain Energy Technol Assess 2014;7:147-53.

[139] Chang K-H, Lin G. Optimal design of hybrid renewable energy systems using simulation optimization. Simul Model Pract Theory 2015;52:40-51.

[140] Mukhtaruddin RNSR, Rahman HA, Hassan MY, Jamian JJ. Optimal hybrid renewable energy design in autonomous system using Iterative-Pareto-Fuzzy technique. Int J Electr Power Energy Syst 2015;64:242-9.
[141] Abdelhak BJ, Najib E, Abdelaziz H, Hnaien F, Yalaoui F. Optimum sizing of hybrid PV/Wind/Battery Using Fuzzy-Adaptive Genetic Algorithm in real and average battery service life. In: International symposium on power electronics, electrical drives, automation and motion; 2014. p. 871-76.

[142] Lujano-Rojas JM, Dufo-López R, Bernal-Agustín JL. Probabilistic modelling and analysis of stand-alone hybrid power systems. Energy 2013;63:19-27.

[143] Hong Y-Y, Lian R-C. Optimal sizing of hybridwind/PV/diesel generation in a stand-alone power system using markov-based genetic algorithm. IEEE Trans Power Delivery 2012;27:640-7.

[144] Askarzadeh A. A discrete chaotic harmony search-based simulated annealing algorithm for optimum design of PV/wind hybrid system. Sol Energy 2013;97:93-101.

[145] Maleki A, Khajeh MG, Rosen MA. Weather forecasting for optimization of a hybrid solar-wind-powered reverse osmosis water desalination system using a novel optimizer approach. Energy 2016;114:1120-34.

[146] Khatib T, Mohamed A, Sopian K. Optimization of a PV/wind micro-grid for rural housing electrification using a hybrid iterative/genetic algorithm: Case study of Kuala Terengganu, Malaysia. Energy Build 2012;47:321-31.

[147] Tahani M, Babayan N, Pouyaei A. Optimization of PV/Wind/Battery standalone system, using hybrid FPA/SA algorithm and CFD simulation, case study: Tehran. Energy Convers Manage 2015;106:644-59.

[148] Connolly D, Lund H, Mathiesen BV, Leahy M. A review of computer tools for analysing the integration of renewable energy into various energy systems. Appl Energy 2010;87:1059-82.

[149] (1 Oct). Webinar minigrid homer energy. Available: <https://cleanenergy solutions.org/sites/default/files/documents/webinar-minigrid-homer-energy. pdf>.

[150] (2016, 1 Oct). Homer Energy. Available: <http://www.homerenergy.com/ HOMER_pro_modules.html>.

[151] P. Lilienthal. (1 Oct). Homer Energy. Available: <https:// d2oc0ihd6a5bt.cloudfront.net/wp-content/uploads/sites/837/2016/03/PeterLilienthal-HOMER-Pro-Software.pdf>.

[152] Khalid F, Aydin M, Dincer I, Rosen MA. Comparative assessment of two integrated hydrogen energy systems using electrolyzers and fuel cells. Int J Hydrogen Energy 2016;41:19836-46.

[153] Baghdadi F, Mohammedi K, Diaf S, Behar O. Feasibility study and energy conversion analysis of stand-alone hybrid renewable energy system. Energy Convers Manage 2015;105:471-9.

[154] Olatomiwa L, Mekhilef S, Ohunakin OS. Hybrid renewable power supply for rural health clinics $(\mathrm{RHC})$ in six geo-political zones of Nigeria. Sustain Energy Technol Assess 2016;13:1-12.

[155] Maatallah T, Ghodhbane N, Ben Nasrallah S. Assessment viability for hybrid energy system (PV/wind/diesel) with storage in the northernmost city in Africa, Bizerte, Tunisia. Renew Sustain Energy Rev 2016;59:1639-52.

[156] Hossain M, Mekhilef S, Olatomiwa L. Performance evaluation of a stand-alone PV-wind-diesel-battery hybrid system feasible for a large resort center in South China Sea, Malaysia. Sustain Cities Soc 2017;28:358-66.

[157] Bentouba S, Bourouis M. Feasibility study of a wind-photovoltaic hybrid power generation system for a remote area in the extreme south of Algeria. Appl Therm Eng 2016;99:713-9.

[158] (2017, 3 March). Hybrid Optimization by Genetic Algorithms software. Available: <http://personal.unizar.es/rdufo/>.

[159] Dufo-López R, Bernal-Agustín JL. A comparative assessment of net metering and net billing policies. Study cases for Spain. Energy 2015;84:684-94.

[160] Dufo-López R, Lujano-Rojas JM, Bernal-Agustín JL. Comparison of different lead-acid battery lifetime prediction models for use in simulation of standalone photovoltaic systems. Appl Energy 2014;115:242-53.

[161] Schiffer J, Sauer DU, Bindner H, Cronin T, Lundsager P, Kaiser R. Model prediction for ranking lead-acid batteries according to expected lifetime in renewable energy systems and autonomous power-supply systems. J Power Sources 2007;168:66-78.

[162] Dufo-López R, Bernal-Agustín JL, Yusta-Loyo JM, Domínguez-Navarro JA Ramírez-Rosado IJ, Lujano J, et al. Multi-objective optimization minimizing cost and life cycle emissions of stand-alone PV-wind-diesel systems with batteries storage. Appl Energy 2011;88:4033-41.

[163] Muhsen DH, Ghazali AB, Khatib T. Multiobjective differential evolution algorithm-based sizing of a standalone photovoltaic water pumping system. Energy Convers Manage 2016;118:32-43.

[164] Abbes D, Martinez A, Champenois G. Eco-design optimisation of an autonomous hybrid wind-photovoltaic system with battery storage. IET Renew Power Gener 2012;6:358-71.

[165] Vergara PP, Rey JM, Silva LCPd, Ordóñez G. Comparative analysis of design criteria for hybrid PV/wind/battery systems. IET Renew Power Gener 2016.

[166] Perera ATD, Attalage RA, Perera KKCK, Dassanayake VPC. A hybrid tool to combine multi-objective optimization and multi-criterion decision making in designing standalone hybrid energy systems. Appl Energy 2013;107:412-25.

[167] Dufo-López R, Cristóbal-Monreal IR, Yusta JM. Stochastic-heuristic methodology for the optimisation of components and control variables of PV-wind-diesel-battery stand-alone systems. Renewable Energy 2016;99:919-35.

[168] Maheri A. Multi-objective design optimisation of standalone hybrid wind-PVdiesel systems under uncertainties. Renewable Energy 2014;66:650-61.

[169] Perera ATD, Attalage RA, Perera KKCK, Dassanayake VPC. Designing standalone hybrid energy systems minimizing initial investment, life cycle cost and pollutant emission. Energy 2013;54:220-30. 


\section{Chapter 2}

\subsection{Part B: Shipboard Power Systems: Configurations and Power Management}

This subchapter provides a review on shipboard power system configurations, control, and power management. The aim is to identify the most suitable configuration in terms of fuel consumption and review the PMSs used in shipboard power systems. This subchapter has been published in the Journal of Energies. The citation of the article is:

Al-Falahi, M., Tarasiuk, T., Jayasinghe, S., Jin, Z., Enshaei, H., \& Guerrero, J. (2018). AC ship microgrids: control and power management optimization. Energies, 11(6), 1458. 


\title{
AC Ship Microgrids: Control and Power Management Optimization
}

\author{
Monaaf D. A. Al-Falahi ${ }^{1}$ (i), Tomasz Tarasiuk ${ }^{2}$ (D), Shantha Gamini Jayasinghe ${ }^{1, *(1)}$, \\ Zheming Jin ${ }^{3}$, Hossein Enshaei ${ }^{1}$ (D) and Josep M. Guerrero ${ }^{3}$ (I) \\ 1 National Centre for Ports and Shipping, Australian Maritime College, University of Tasmania, \\ Tasmania 7248, Australia; monaaf.alfalahi@utas.edu.au (M.D.A.A.-F.); hossein.enshaei@utas.edu.au (H.E.) \\ 2 Department of Marine Electrical Power Engineering, Gdynia Maritime University, Gdynia 81225, Poland; \\ t.tarasiuk@we.am.gdynia.pl \\ 3 Institute of Energy Technology, Aalborg University, Aalborg 9100, Denmark; zhe@et.aau.dk (Z.J.); \\ joz@et.aau.dk (J.M.G.) \\ * Correspondence: shanthaj@utas.edu.au; Tel.: +61-3-6324-9752
}

Received: 22 March 2018; Accepted: 4 June 2018; Published: 5 June 2018

\begin{abstract}
At sea, the electrical power system of a ship can be considered as an islanded microgrid. When connected to shore power at berth, the same power system acts as a grid connected microgrid or an extension of the grid. Therefore, ship microgrids show some resemblance to terrestrial microgrids. Nevertheless, due to the presence of large dynamic loads, such as electric propulsion loads, keeping the voltage and frequency within a permissible range and ensuring the continuity of supply are more challenging in ship microgrids. Moreover, with the growing demand for emission reductions and fuel efficiency improvements, alternative energy sources and energy storage technologies are becoming popular in ship microgrids. In this context, the integration of multiple energy sources and storage systems in ship microgrids requires an efficient power management system (PMS). These challenging environments and trends demand advanced control and power management solutions that are customized for ship microgrids. This paper presents a review on recent developments of control technologies and power management strategies proposed for AC ship microgrids.
\end{abstract}

Keywords: droop control; hierarchical control; isochronous control; power management; ship microgrids

\section{Introduction}

Diesel engine driven or gas turbine driven generators are the sources found in conventional ship microgrids, which are generally known as gensets. They are controllable sources and thus their power levels can be adjusted to meet the required demand. With the growing demand for emission reduction and fuel efficiency improvements these conventional gensets must be supplemented with alternative energy sources such as wind, solar, and fuel cells [1-4]. Even though there are different opinions on solar and wind installations on shipboards, as their contribution to the power generation is not significant compared to gensets, in certain ship types, the contribution can be non-marginal, and thus a growing trend can be observed in research and relevant technology development in these areas $[5,6]$. Out of these alternative energy sources, the fuel cell has been identified as the most promising technology for ships [4,7]. In addition to the incorporation of alternative energy sources, energy recovery technologies are increasingly being introduced into ship microgrids aiming towards improving the fuel efficiency [8]. For example, waste heat recovery systems that utilize exhaust fumes for electricity production are able to improve main engine efficiency by approximately $5 \%$, which greatly reduces emissions and fuel cost $[3,9]$. 
Even though these alternative energy technologies help improve fuel efficiency and reduce emissions, their intermittency and / or slow response make energy storage technologies such as batteries or supercapacitors essential to ensure reliable operation and fast response [10]. Moreover, the presence of pulse loads, such as radar, may exceed the ship's rated generation capacity, leading to unstable operation [11-13]. This makes energy storage inevitable in shipboard power systems to meet fast transient characteristics [4,14-17]. Therefore, future ship power systems will include traditional gensets, alternative energy sources, energy storage technologies, and energy recovery systems.

Together with the aforementioned technologies, the ship power system can be considered as a typical islanded microgrid when the ship is at sea. The same power system can be considered as a grid connected microgrid or an extension of the shore power grid when the ship is at berth. Thus, ship microgrids show some resemblance to terrestrial microgrids [18-20]. Nevertheless, the major difference comes from the way the load and source dynamics are distributed in each system. In terrestrial microgrids, renewable energy sources account for a large share of power and thus they bring associated intermittencies into the power generation system while the loads show relatively small and slow changes [21-23]. In contrast, the main sources in ship microgrids are controllable, while the loads, such as propulsion loads, are highly dynamic. In addition, the presence of large power electronic loads is another major difference in ship microgrids, which results in serious power quality issues compared to terrestrial microgrids $[2,24]$. Therefore, despite some similarities, ship microgrids require special attention in research and associated technology developments.

The major challenge with such islanded microgrids is matching the source dynamics to that of the loads while ensuring robust operation and fast response [25]. Control and power management strategies play a vital role in achieving these objectives [26-33]. Droop and isochronous are the commonly used primary control technologies in AC or DC ship microgrids [27]. On top of these controllers, there should be a centralized or decentralized power management controller to coordinate power generation. Hierarchical control, which is one of the popular approaches reported in the literature for power management and the control of islanded microgrids, can be adopted for ship microgrids as well [26]. Three-level hierarchical control is the most common scheme, and in this scheme the primary control focuses on individual units while the secondary and tertiary controls focus on system level control and power management, respectively $[20,26]$. In contrast to the common centralized control approach, distributed control, especially with multi-zonal architectures in emerging ship power systems, enables robust operation even during faults in part of the ship microgrid [34,35]. The aim of this paper is to present a review of the advancements of architectures, control technologies, and power management strategies of ship microgrids. Moreover, the author's original research on the performance of droop control based power sharing, at different operating conditions, are presented. This paper serves as a useful reference for academic researchers and practicing engineers in the field of ship microgrids.

\section{Shipboard AC Power System Architectures}

Ship microgrids generally follow the shore practice and thus, $400 \mathrm{~V} / 50 \mathrm{~Hz}$ or $440 \mathrm{~V} / 60 \mathrm{~Hz}$ three phase low voltage (LV) AC distribution systems are common in a majority of the ships. This allows normal industrial equipment, which designed to withstand harshness at sea, to be used on ships. This equipment generally constitutes ships auxiliary service loads. Compared to these service loads, propulsion loads are very high, and thus the LV distribution system is not sufficient to cater to these loads. Therefore, high voltage (HV) three-phase distribution systems, such as $3.3 \mathrm{kV}, 6.6 \mathrm{kV}$, and $11 \mathrm{kV}$, are used to transmit power to these loads. Owing to these differences in voltage levels, power requirements and dynamics, propulsion, and service loads were segregated in early electric ships [3]. Nevertheless, due to the fact that finding solutions to the above challenges is more beneficial than maintaining two systems, the trend has now been shifted from segregated power systems to integrated power systems (IPSs). 
The history of surface ship electric propulsion is dated to the beginning of the last century, when this system was installed onboard the U.S.S. Jupiter, followed by other vessels, most prominently passenger ships, like the Queen Elizabeth II (QEII) [3]. QEII uses an IPS where propulsion loads are fed through the HV distribution system and service loads are fed through the LV distribution system, as shown in Figure 1a. The LV bus is fed from the HV bus through a step down transformer [36]. In this architecture, all the generators are connected to a single HV bus, which run the risk of blackout if there is a failure at the HV side. As a solution, instead of having a single HV bus, the two HV/LV radial bus architectures, shown in Figure $1 b$, have been used in many ships $[37,38]$. The two busses, generally referred to as the port side bus and starboard side bus, are linked with bus-tie switches. These switches can be opened to disconnect the faulty bus from the healthy bus in the event of a fault and thus potential blackouts can be prevented. Moreover, owing to the progressive development in power electronics devices, the integration of more-electric technologies (METs) is gaining more attention in the marine industry. In this context, with the growing demand for emission reductions and fuel efficiency improvements, the integration of renewable energy sources and energy storage systems is becoming popular in the maritime industry. A typical arrangement for integrating such systems into a shipboard power system is shown in Figure 1b [39].

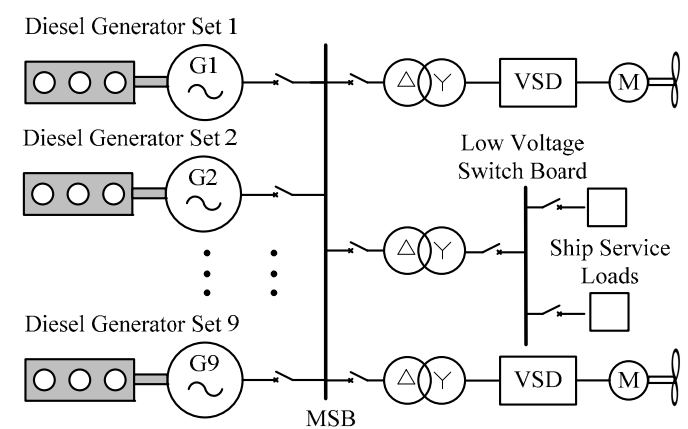

(a)

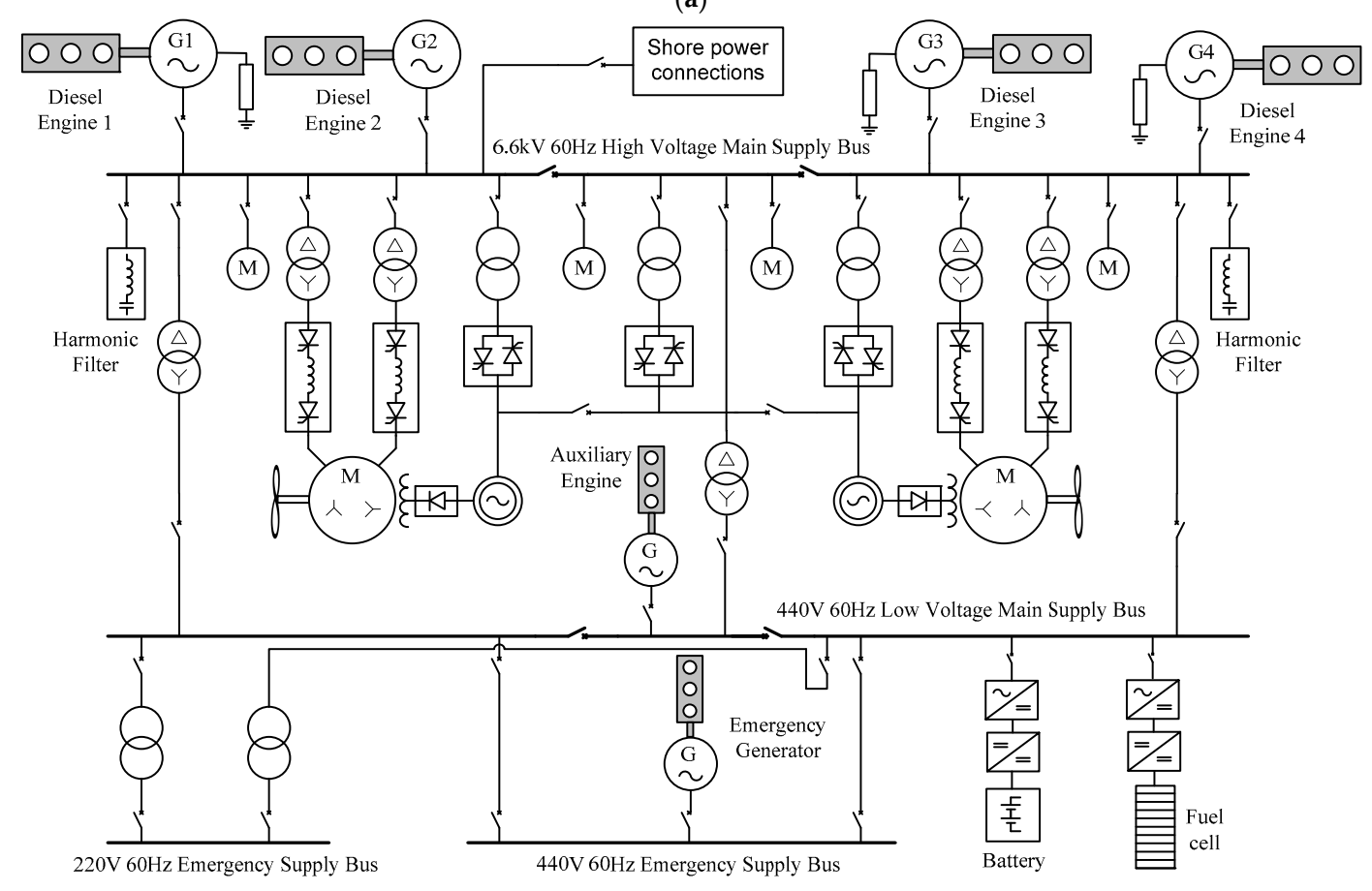

(b)

Figure 1. (a) Power system architecture of the Queen Elizabeth II cruise ship; (b) Example of a radial AC distribution system. (M: motor, MSB: main switchboard, VSD: variable speed drive). 
Similar to QEII, the LV side of the radial distribution system is also supplied by the HV side through transformers. But, unlike in QEII, the LV side is also divided into two busses linked through bus tie switches in the radial system. This helps isolate LV buses as well in the event of a fault. Moreover, there is an auxiliary generator, which can be used to feed the LV bus if the power from the $\mathrm{HV}$ side is insufficient or unavailable. In addition, the emergency power supply, shown in Figure 1b, is a requirement under safety of life at sea (SOLAS) regulations, which should be available for emergency lighting, alarms communications, water tight doors, and other services that are necessary to maintain safety in the event of main power failure [38]. This can be a battery bank, a generator, or both.

Even in the radial distribution system, there could be possibilities for losing power to the essential loads, such as propulsion motors, in the event of a fault in a HV bus. Moreover, certain sections might not be able to isolate without affecting some of the essential loads attached to it [35]. As a solution, modern electric ships tend to use zonal electrical distribution system (ZEDS) architecture based IPSs over radial architecture [40,41]. The principle feature of ZEDSs is that the entire network is split into a few sections (IEEE Std 45.3-2015 [42]), as shown in Figure 2, called zones, which are connected through bus-tie switches. Each zone has its own load center, which is powered by generating sources. All the zones are connected by a starboard bus and a port bus. One of those buses is located above the water line, while the other is positioned below to increase the distance between them, and to reduce possible damages to both busses in case of fault [40].

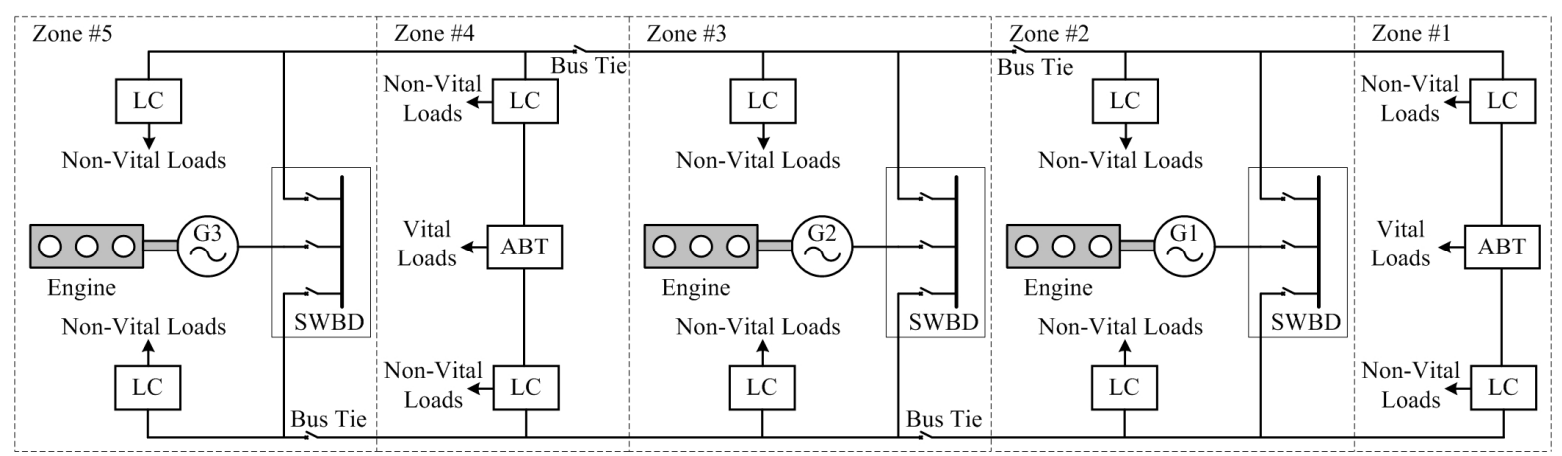

LC - Load Centre, ABT - Automatic Bus Transfer, G - Generator, SWBD - Switchboard

Figure 2. Notional AC zonal electrical distribution system.

Ship power systems are generally ungrounded. This is to limit the risk of system collapse in the event of a single fault. Nevertheless, HV systems inevitably lead to the increased risk of transient overvoltage due to a phase-to-earth arc flash. Therefore, instead of ungrounded systems, ships with HV distribution systems use high resistance grounding [43].

\section{Control Technologies}

Power generation in ship microgrids is dominated by synchronous generators, which are controllable. However, the loads are highly dynamic and, in certain cases, may contain unpredictable fast changes. Therefore, in order to manage complexities and achieve desired control objectives, the hierarchical framework, which is shown in Figure 3, can be adopted forship microgrids as well [25,44]. Moreover, as explained below, the existing control technologies in ship power systems can also be described in line with the hierarchical control framework. In hierarchical control, the primary level objective is to achieve load sharing among the power sources. The secondary level control objective is to secure bus signals at their nominal values. The tertiary level control is used to achieve optimal operation with intentional objectives [26]. In this scheme, the higher the level of control, the slower the regulation it provides. Moreover, the scope of the control widens as the level increases. 


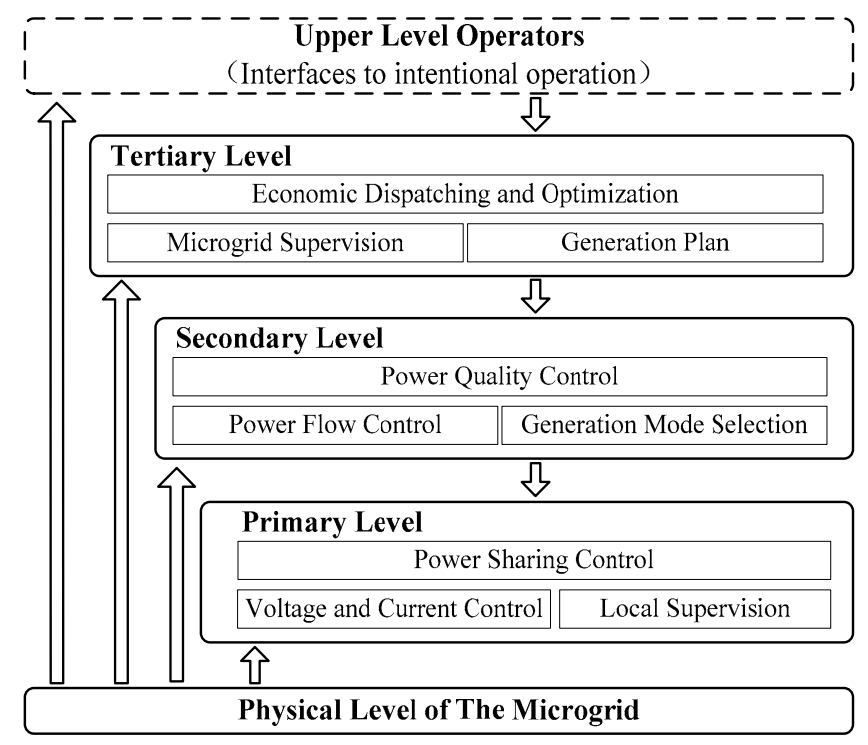

Figure 3. Hierarchical control framework.

\subsection{Isochronous Control}

The majority of shipboard power systems are AC distribution systems, where frequency and voltage are the two fundamental parameters to be maintained within specified limits. Since diesel engine or gas turbine driven synchronous generators are the commonly used sources in these systems, the governor controller regulates the frequency while the automatic voltage regulator (AVR) regulates the voltage. The governor, which comes in the form of a hydro-mechanical controller or an electronic controller, controls the fuel supply to the engine and in turn regulates the speed, ( $\omega)$, of the rotor. The AVR comes as an electronic controller and it controls the current supplied to the field winding of the generator, which in turn regulates the output voltage, (V). Simplified block diagrams of these two controllers are shown in Figure 4.

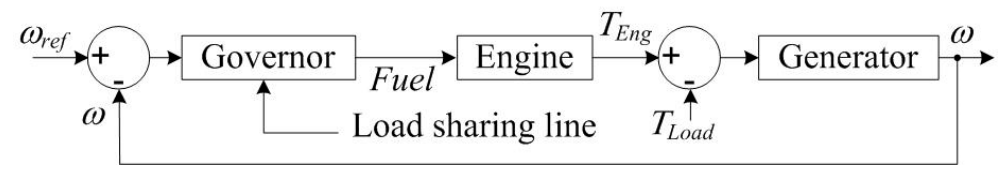

(a)

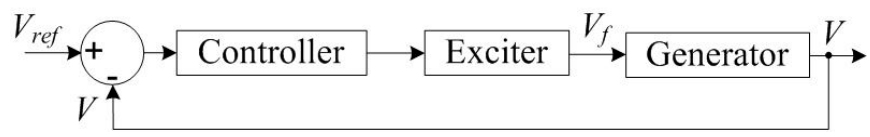

(b)

Figure 4. (a) Isochronous speed controller; (b) automatic voltage regulator.

The controller shown in Figure $4 \mathrm{a}$ is known as an isochronous speed controller, as it regulates the speed of the engine at the set point [45]. Under isochronous control, both the voltage and frequency of the generator output are maintained at set points irrespective of changes in the load. In comparison to the hierarchical control framework, the isochronous control for a single generator falls within the scopes of primary and secondary levels as it regulates the bus voltage and frequency at set points, while supplying the demanded power.

The aforementioned isochronous control works well for a single generator. If two or more generators are connected to the same power bus, one of the engines may try to take the entire load 
while the others might not take the load. This leads to instabilities and may result in blackout. Therefore, in order to solve this issue, communication between the governor controllers, in the form of a load sharing line or a communication link, such as controller area network (CAN) bus or Field bus, is required [46]. With the help of the communication link, each engine can be set to take a specific share of the load without going into extremes or instabilities. In this configuration, the power management system (PMS), which determines the power reference for each engine, can be attributed to the tertiary level of the hierarchical control framework.

Even though isochronous load sharing is capable of regulating voltage and frequency at set points, it has not become the popular choice in ship microgrids, mainly due the harshness of the environment in ships, which adversely affects communications. Moreover, in order to implement isochronous power sharing, all the governor controllers should be compatible, and most of the cases should come from the same manufacturer, which may not be possible in some cases. Even though solutions such as advanced generator supervision (AGS) have been developed to prevent blackout in faulty situations, isochronous control is still not the popular choice when it comes to very large shipboard power systems [47].

\subsection{Droop Control}

Compared to isochronous control, droop control is the popular choice for power sharing in multi-generator shipboard power systems, as it does not require communication between the governor controllers. In contrast to the fixed frequency and fixed voltage operation in the isochronous control, droop control lets the frequency and voltage vary in proportion to the active, $(\mathrm{P})$, and reactive power, $(\mathrm{Q})$, demands of the load. The corresponding speed and voltage controllers are shown in Figure 5, where the speed and voltage references are reduced linearly as the active and reactive power demands increase.

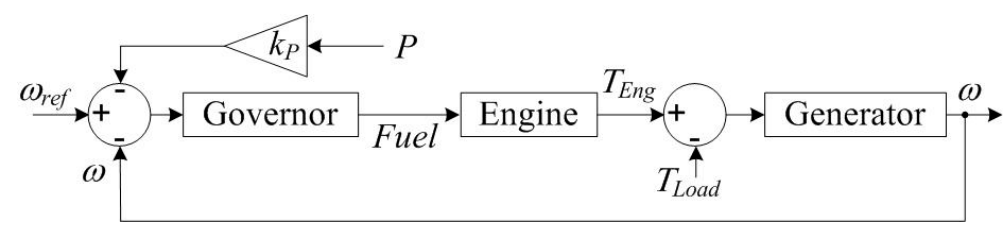

(a)

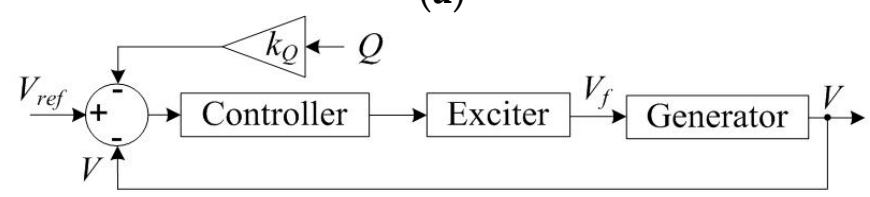

(b)

Figure 5. Engine-generator control system (a) speed droop and (b) voltage droop.

The droop control matches with the inherent $P / f$ droop nature of synchronous machines where loads on the electrical side slows down the rotor and as a result, speed drops [48]. The governor injects more fuel in response to the speed drop and thus, as shown in Figure 6a, the genset becomes stable at a new frequency, which is lower than the nominal frequency, $f_{0}$. Not only the frequency but also the voltage settles at a new value in the same way when there is a change in the reactive power demand [45]. In a multi generator system, each governor senses the speed drop, supplies more power to the grid and finally settles at a new frequency. The amount of power supplied by each genset depends on the droop settings of the genset. If the settings are equal, all the generators equally share the load. Moreover, the droop control can be applied for power converter based systems as well. However, irrespective of the system, droop based power sharing falls within the scope of the primary response in the hierarchical control scheme [48]. 


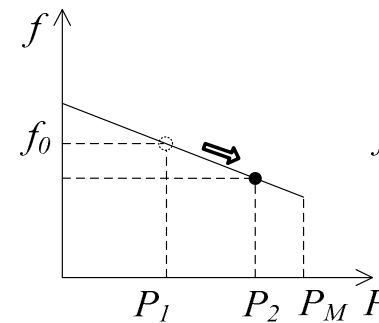

(a)

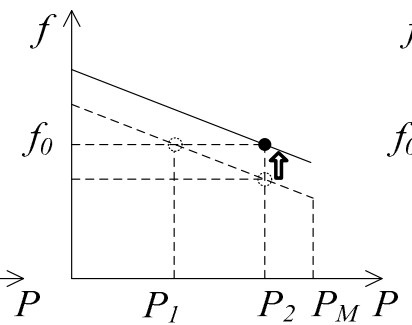

(b)

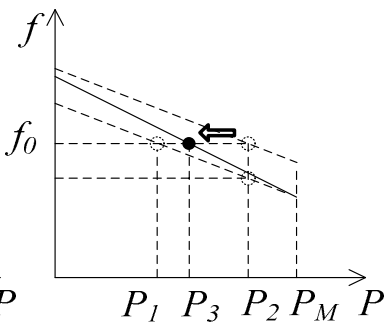

(c)

Figure 6. Speed droop control (a) primary response; (b) secondary response; (c) tertiary response.

In a multi-generator system, the secondary control can be used to bring the frequency back to the nominal value, as shown in Figure $6 b$, by adding an offset to the speed reference, $\omega_{\text {ref }}$. This is known as the secondary response that can be attributed to the level 2 control of the hierarchical control scheme. As shown in Figure $6 c$, further changes can be made to the droop controller by changing the droop gain, $k_{p}$, which changes the power levels of each engine to their best possible levels under the given condition. This tertiary response belongs to the level 3 control of the hierarchical scheme. Similar to the abovementioned speed, droop controllers operate based on the reactive power demand [26,48]. According to the rules of most of ship classification societies, the proportionality of load sharing has to be within the range of $\pm 15 \%$ of the rated active power and $\pm 10 \%$ of the rated reactive power of the largest generator [3,49-51].

In contrast to isochronous control, droop control requires only local measurements of voltage and frequency, and thus, it allows multiple generators to share the load without hunting and without the need of inter-unit communication. This makes droop control robust, highly reliable, and flexible in adding/removing the generators of different power ratings to the grid [52,53]. Nevertheless, since the active and reactive power supplied by generators depend on frequency and voltage deviations, large loads result in increased deviations, and this is an inherent trade-off of droop control [39,53-55].

\subsection{Application of Droop Control-Tests in a Real Ship}

In order to exemplify the real system behavior of droop control under highly non-linear loading conditions, the results of an experiment conducted in a ship are presented in Figure 7. During the experiment, two generators of $301 \mathrm{~kW}(376 \mathrm{kVA})$ rated power worked in parallel. The generators were driven by diesel engines, each having $357 \mathrm{~kW}$ of rated power. Mechanical-hydraulic governors were used to control the shaft speed of the prime movers. Despite the medium rated power of the gensets, they displayed the typical behavior of a ship electric power plant with a droop control scheme. The most significant load was the bow thruster drive with a rated power of $125 \mathrm{~kW}$, supplied via a power converter. This bow thruster should be considered as a highly non-linear load.

The frequency droop is clearly visible in Figure $7 \mathrm{~b}$. It enables fast and satisfactory load sharing between the generators. The proportionality of active power sharing is shown in Figure 7c, which is within the limits of ship classification societies. It is worth noting here that this observation was made despite having slight differences in the characteristics of the two governors. The reactive power supplied by each generator is shown in Figure $7 \mathrm{~d}$. Even though the sharing of reactive power is disproportional, the difference is small in comparison to rated power and thus the relative sharing is safely within $2.8-3.2 \%$ of the rated reactive power of each generator. Furthermore, as shown in Figure 7e, it can be seen that the load increase results in an increase in the bus voltage. It is due to the impact of a concurrent increase in the levels of voltage and generator current distortions and their impact on the AVR's work. The voltage of the total harmonic distortion (THDu) during the process increased from $0.9 \%$ to $4.5 \%$, and the current THD increased from $1.7 \%$ to $16.7 \%$. The impact of the distortions on the work of AVRs on the same ship was investigated during other research [56]. It was found out that $T H D u=3.7 \% U=399.7 \mathrm{~V}, \mathrm{THDu}=4.8 \% U=400.4 \mathrm{~V}$, and $T H D u=7.9 \% U$ 
$=401.9 \mathrm{~V}$. Thus, reactive load sharing by droop control can be adversely affected by voltage and current distortions.

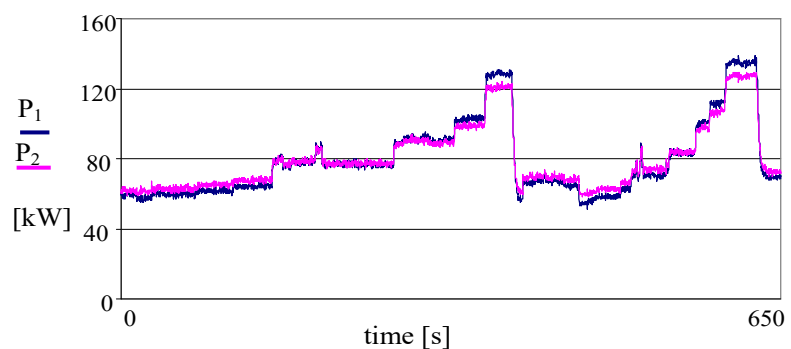

(a)

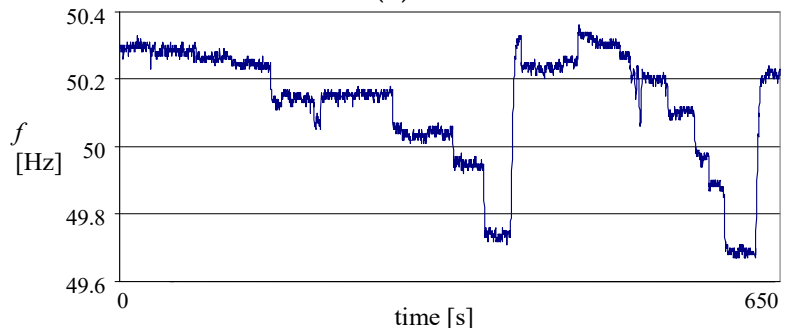

(b)

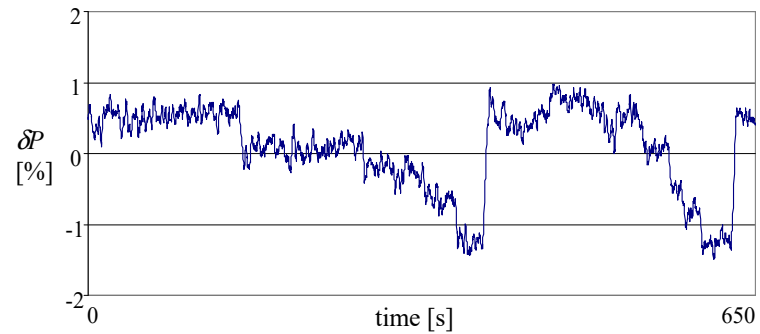

(c)

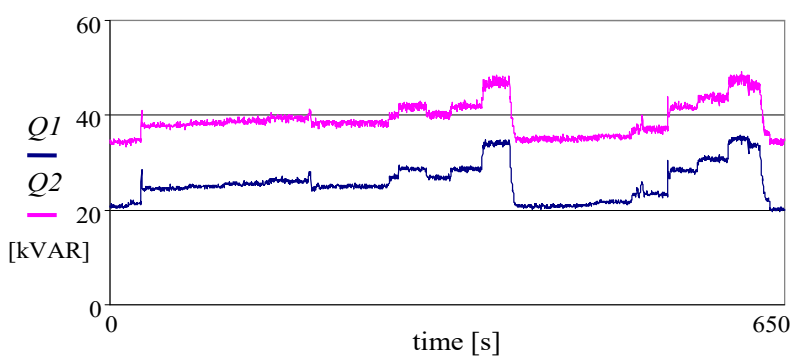

(d)

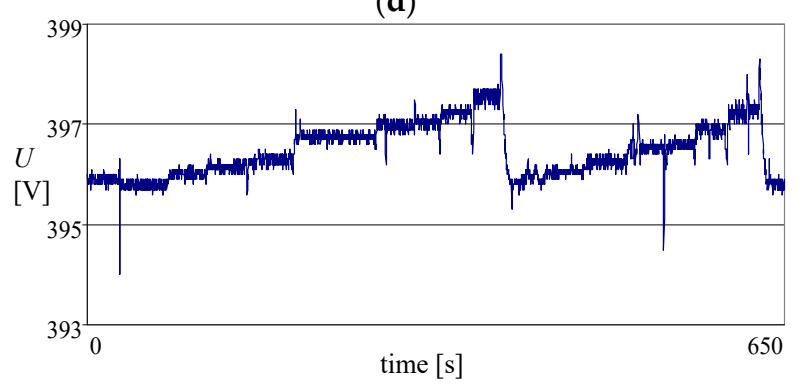

(e)

Figure 7. Variations of the basic parameters when two generators are running in parallel: (a) active power; (b) frequency; (c) proportionality of active power sharing; (d) reactive power; (e) voltage on main bus bars. 


\subsection{Grid-feeding Power Converter Control}

As discussed in the introductory section, the ever growing trend for low emission technologies and the demarcation of the emission controlled areas (ECAs) resulted in a trend to incorporate more renewable energy technologies, such as fuel cells, photovoltaic (PV) power systems, wind energy conversion systems, and energy storage technologies such as batteries and supercapacitors [4]. Nevertheless, due to relatively low power levels these technologies cannot perform as grid-forming sources. Therefore, the corresponding grid connecting inverters are often used as a grid feeding converter where the converter injects a specific amount of power to the grid depending on the output of the maximum power point tracking algorithm [57] or the command from the ship PMS [48]. Current control is preferred in this mode of operation and thus the interfacing converter can be considered as a current source. Batteries and supercapacitors are used to absorb power fluctuations and thus they work mostly at transient conditions. Therefore, their power reference is generally derived from voltage/frequency stabilization algorithms.

A typical grid-feeding converter controller block diagram is shown in Figure 8. The inner controller consists of a fast current control loop that regulates the current, $i$, injected into the grid and thus the power delivered to the objectives $[48,53]$. The synchronous reference frame based $d q$-frame control is often used in these inner current control loops. Nevertheless, unbalanced grid conditions and the presence of harmonics degrades the performance of the synchronous reference based control. Proportional resonant (PR) controllers solve the harmonic issue with properly tuned resonant to suppress the effects of harmonics [58]. Moreover, the natural reference frame $(a b c)$ based controllers, realized in the form of proportional integral (PI), PR, and hysteresis for dead-beats, can be used to control the grid feeding converter [59].

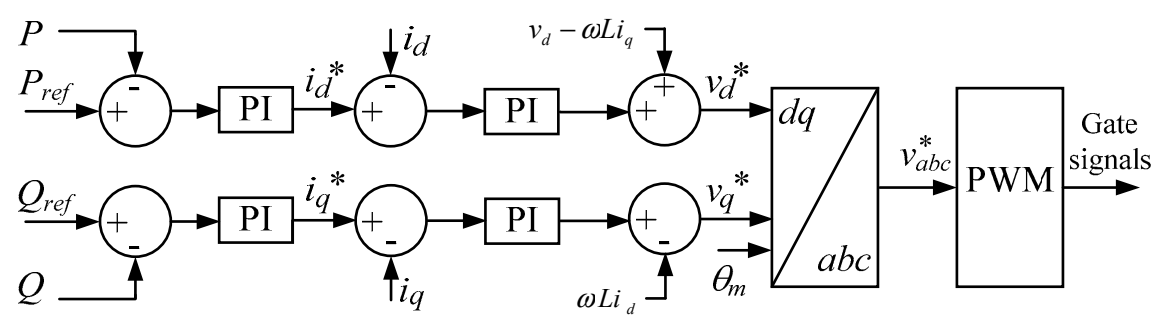

Figure 8. Grid feeding inverter controller.

\section{Power Management Optimization}

Ship PMS plays a vital role in maintaining the power balance, improving fuel efficiency, preventing blackouts, and ensuring safe operation at various operating conditions. The broader scope of the PMS includes power saving, control of propulsion machinery, control of main and emergency generators, loading and unloading of generator alternator sets, load dependent start/stop, load sharing, load shedding, motors automatic blocking, power and frequency control, synchronizing, and monitoring and load analysis illustration. These functionalities fall within the secondary, tertiary, and upper level control of the aforementioned hierarchical control scheme. Some of these functions are explained in detail below.

- Energy saving: Energy savings can be presented in the three following ways: reduction in specific fuel consumption (SFC), reduction in propulsion fuel consumption, and reduction in overall vessel fuel consumption.

- Automatic start/stop/standby of auxiliary generators: Generators are operated depending on power consumption. A surplus of available power should be limited as much as possible from safety point of view. The PMS constantly compares the total generator load against the load dependent automatic start/stop limits. If the available power minus safety margin is less than 
the required power, either due to increase in load or fault in a running generator set, the PMS will automatically start the next standby generator set in the start sequence. When the load decreases to a level that will not overload the remaining generators, the standby generator will stop and disconnect.

- Automatic load sharing: When the load increases, another generator is connected to the switchboard. PMS divides the load in an optimal manner on generators after synchronizing.

- Load shedding: When a sudden loss of a generator or load increase occurs, leading to an overload of other generators, non-essential loads are automatically disconnected by the PMS. For example, thrusters can operate with reduced load in dynamic positioning for a period of time because of the slow response of the ship with respect to position and handling. This period is sufficient to get the next unit on-line and increase the power generating capacity. According to [50], the PMS "is to prevent overloading of the generators and maintain power to the essential loads such as propulsion load by shedding non-essential loads."

- Automatic synchronizing and system restoration: Automatic synchronizing is performed in order to ensure generators are running at required speed, voltage, and phase. After a blackout, the system is required to follow the sequence control of a start-up and reconfiguration of the power system, which includes starting and synchronizing generator sets and sequential starts of loads.

- Monitoring and load analysis illustration: The PMS consist of a monitoring system to monitor the load profile, active and reactive load sharing monitoring to monitor the load sharing failure, fuel consumption monitoring, graphically displayed information that can help operators to target wasted energy, and engine performance monitoring. Additionally, some PMS monitoring systems provide historical data to help make decisions on the maintenance and operation of machinery and other ship power system components [48].

- Load transfer: The PMS can control and monitor the load transfer from shaft to auxiliary and vice versa in hybrid electric ships, and shore power to auxiliary in cold ironing [60].

The load type and condition plays a vital role in determining the efficiency of power management for vessels. Hence, the appropriate PMS can be fitted based on the types of loads present onboard the ship and their dynamics. For tanker ships, pumps and compressors are significant factors as they consume a significant portion of the generated power. In cruise ships, approximately $50 \%$ of total fuel consumption is consumed by hotel loads such as air conditioning systems, heating systems, galley equipment, stage equipment, and lights. For container ships, cargo handling equipment plays a dominant role and defines the special power requirement characteristics of the installed power system onboard. Moreover, in certain vessels, ballast water pumps present a large load on the ship power system.

Similar to the terrestrial microgrids, power management in a ship's microgrid can also be implemented in centralized or decentralized manners. A centralized management system requires computation resources and data gathered from internal microgrid components in the case of an islanded microgrid, and from external components in the case of a grid connected microgrid. Centralized PMS, used to achieve the minimum operational costs with efficient operation, gives the advantages of wide observation of the whole system and this type of system is easy to implement [61]. However, a single point of failure in the centralized PMS will affect the entire system [62]. On the other hand, decentralized PMS is preferred when more flexibility in operation and a non-single point of failure system is required. Due to the dynamic nature and finite generation inertia associated with IPSs, decentralized PMS is preferred to achieve the balance between generation and load in real time while satisfying the operational constraints [63-65].

With the incorporation of alternative energy technologies, energy recovery technologies, and energy storage systems, ship PMS becomes a key element in optimizing energy usage and thereby improving fuel efficiency. In contrast to terrestrial microgrids, there are many constraints associated with the optimization of ship PMS as it is heavily influenced by dynamic loads [66]. 
Moreover, the objectives of the optimal power management for ship microgrids depend on the operating conditions of the ship, which can be generally classified into emergency, alert, restorative, reconfigurative, cruise, acceleration, deceleration, and docking [25,67].

Researchers have proposed various optimal power management techniques using classical and meta-heuristic optimization methods by considering the minimization of operational costs and greenhouse gas emissions as their main objective function [68-71]. In [11], the authors have proposed a model predictive control (MPC) based energy management strategy in order to optimally operate the system by dealing with power ramp rate problems for all-electric ships (AES). The proposed hybrid EMS is a combination of heuristics and MPC in which heuristics are applied to distinguish the system's state transitions and MPC is applied to fulfill the control objective function in each state. Another study, reported in [72], proposed an adaptive MPC for AES energy management, which provides better energy management compared to the use of MPC alone. In [73], the authors have proposed a multi-objective optimization with real-time MPC for electric ships. The results revealed that the use of the proposed method provides less energy storage losses than MPC. In [74], authors proposed a fuzzy-based particle swarm optimization (FPSO) as a power management strategy for ship electric power systems, comprising integrated full electric propulsion, energy storage, and shore power supply. The main multi-objective function of the optimization is to minimize operating cost and greenhouse gas emissions. The proposed method provides better results in terms of minimum operational costs and greenhouse gas emissions compared to conventional PSO. In other studies, Genetic Algorithm (GA) is used to solve optimization problems including reconfiguration and restoration in ship power systems [75,76]. In other studies, trim optimization is used to reduce the fuel cost and emissions by minimizing fuel consumption [77]. Moreover, LINDO optimization software is used to achieve restoration in ship power system by maximizing the restored load and giving priority to vital loads [41]. Biogeography based optimization (BBO) and particle swarm optimization (PSO) are other techniques that can be used in ship microgrids. Out of these methods, GA has been recognized as a more reliable solution for optimal DC voltage and power control in medium voltage DC (MVDC) shipboard power systems [78]. Another study reported in [79] proposed a real-time optimization based on PSO to optimally manage the power of notional MVDC system for a DC ship microgrid.

The multi-agent system (MAS) technique is one of the most advanced and flexible choices in optimal control and power management [80-82], where there are multiple agents interacting with each other in a cooperative manner to solve complex problems effectively [81]. Recent researches have revealed that MAS techniques are able to achieve the minimum demand-supply mismatch, while maximizing the capacity of energized loads, by determining the switch statues of loads in DC zones $[63,64]$. This has further revealed that the system can be operated normally by preventing blackout in the event of a failure in a subsection. In [83], a real-time heterogeneous MAS is proposed to manage power for AES with a DC zonal system. The study found that incorporating real-time control methods with MAS provides better performance than conventional MAS under the frequency load shedding method. Table 1 summarizes recent developments in power management methods proposed for ship microgrids. 
Table 1. Power management methods used in shipboard power systems.

\begin{tabular}{|c|c|c|c|c|c|c|c|c|c|c|c|c|c|c|c|c|c|c|c|c|c|c|c|}
\hline \multirow{2}{*}{ Method } & \multirow{2}{*}{ Objective } & \multicolumn{19}{|c|}{ Constrains* } & \multirow{2}{*}{$\begin{array}{l}\text { Operating } \\
\text { Condition }\end{array}$} & \multirow{2}{*}{$\begin{array}{c}\text { Software/ } \\
\text { Experimental }\end{array}$} & \multirow{2}{*}{ Ref } \\
\hline & & VL & $F$ & SC & PCC & PB & GL & OT & GHG & ss & TD & RR & BP & GSS & ESS & LL & SI & $\mathrm{CC}$ & PG & $\overline{B C}$ & & & \\
\hline $\begin{array}{l}\text { Hybrid heuristics } \\
\text { and MPC based } \\
\text { EMS }\end{array}$ & $\begin{array}{l}\text { Minimizing the cost to manage } \\
\text { the energy of storage system }\end{array}$ & & & & & $\sqrt{ }$ & & & & & & $\sqrt{ }$ & & & $\sqrt{ }$ & & & & $\sqrt{ }$ & & $\begin{array}{l}\text { Ramp rate } \\
\text { conditions }\end{array}$ & $\begin{array}{l}\text { Software and } \\
\text { experimental }\end{array}$ & [11] \\
\hline Adaptive MPC & $\begin{array}{l}\text { Maximize system reliability and } \\
\text { efficiency }\end{array}$ & $\checkmark$ & & & & $\sqrt{ }$ & & & & $\checkmark$ & & & & & $\sqrt{ }$ & & & & $\sqrt{ }$ & & Normal & Software & [72] \\
\hline $\begin{array}{c}\text { Real-time } \\
\text { multi-objective MPC }\end{array}$ & $\begin{array}{l}\text { Minimize the power tracking } \\
\text { error and storage losses }\end{array}$ & $\sqrt{ }$ & & & & & & & & & & & & & $\sqrt{ }$ & & & & & $\sqrt{ }$ & Normal & Software & [73] \\
\hline $\begin{array}{l}\text { Fuzzy-based PSO } \\
\text { (FPSO) }\end{array}$ & $\begin{array}{l}\text { Minimize the operating cost and } \\
\text { GHG }\end{array}$ & & & & & $\sqrt{ }$ & & & & $\sqrt{ }$ & $\checkmark$ & $\sqrt{ }$ & & $\sqrt{ }$ & & & & & & & Normal & Software & [74] \\
\hline $\begin{array}{l}\text { Multi- objective } \\
\text { non-dominated } \\
\text { Sorting Genetic } \\
\text { Algorithm II }\end{array}$ & $\begin{array}{l}\text { Minimize the total power } \\
\text { adjustments, individual active } \\
\text { power set-point adjustments and } \\
\text { individual reactive power } \\
\text { set-point adjustments }\end{array}$ & $\sqrt{ }$ & $\sqrt{ }$ & & & & & & & & & & & & & & $\sqrt{ }$ & $\sqrt{ }$ & & & Normal/alert & Software & [20] \\
\hline \multirow[t]{2}{*}{ Multi-agent } & $\begin{array}{l}\text { Minimize the mismatch between } \\
\text { generation and load and to serve } \\
\text { as many higher priority loads as } \\
\text { possible in operational real time }\end{array}$ & $\sqrt{ }$ & $\sqrt{ }$ & & & & & & & & & & & & & & & & & & Normal/Emergency & Software & [63] \\
\hline & $\begin{array}{l}\text { Maximize capacity of the } \\
\text { energized loads }\end{array}$ & & & $\sqrt{ }$ & $\sqrt{ }$ & & & & & & & & & & & & & & & & Normal/Emergency & Software & [64] \\
\hline Real-time PSO & Minimize the system's cost and & & & & $\sqrt{ }$ & & & & & & & & & & & $\sqrt{ }$ & & & & & $\begin{array}{l}\text { Normal and } \\
\text { pulse load }\end{array}$ & Software & [79] \\
\hline $\begin{array}{c}\text { Dynamic } \\
\text { programming }\end{array}$ & Minimize the total variable cost & & & & & $\sqrt{ }$ & $\sqrt{ }$ & $\sqrt{ }$ & $\sqrt{ }$ & $\checkmark$ & $\sqrt{ }$ & $\sqrt{ }$ & $\sqrt{ }$ & & & & & & & & Cruise ferry & Software & [68] \\
\hline
\end{tabular}


Table 1. Cont.

\begin{tabular}{|c|c|c|c|c|c|c|c|c|c|c|c|c|c|c|c|c|c|c|c|c|c|}
\hline \multirow{2}{*}{ Method } & \multirow{2}{*}{ Objective } & \multicolumn{17}{|c|}{ Constrains * } & \multirow{2}{*}{$\begin{array}{l}\text { Operating } \\
\text { Condition }\end{array}$} & \multirow{2}{*}{$\begin{array}{c}\text { Software/ } \\
\text { Experimental }\end{array}$} & \multirow{2}{*}{ Ref. } \\
\hline & & VL & $\mathbf{F}$ & SC & PCC & PB & GL & OT & GHG & ss & TD & RR & BP & GSS & ESS & $S$ LL $\quad$ SI & $\mathrm{CC}$ & PG $\quad$ BC & & & \\
\hline $\begin{array}{c}\text { Dynamic } \\
\text { programming and } \\
\text { PSO }\end{array}$ & $\begin{array}{l}\text { Minimum operation cost and } \\
\text { GHG emissions limitation }\end{array}$ & & & & & $\sqrt{ }$ & $\sqrt{ }$ & & $\sqrt{ }$ & $\sqrt{ }$ & $\sqrt{ }$ & $\sqrt{ }$ & $\sqrt{ }$ & $\sqrt{ }$ & $\sqrt{ }$ & & & & Cruise ferry & Software & [69] \\
\hline $\begin{array}{l}\text { Recursive searching } \\
\text { algorithm }\end{array}$ & $\begin{array}{l}\text { Minimize fuel cost and GHG } \\
\text { emissions limitation }\end{array}$ & & & & & $\sqrt{ }$ & & & $\sqrt{ }$ & & & $\sqrt{ }$ & $\sqrt{ }$ & $\sqrt{ }$ & & $\sqrt{ }$ & & & Normal & Software & {$[70]$} \\
\hline $\begin{array}{l}\text { Numerical } \\
\text { algorithm }\end{array}$ & Minimize fuel consumption & & & $\sqrt{ }$ & & & & & & & & & & & $\sqrt{ }$ & & & & $\begin{array}{l}\text { Seven operating } \\
\text { conditions }\end{array}$ & $\begin{array}{l}\text { Software and } \\
\text { experimental }\end{array}$ & [84] \\
\hline $\begin{array}{c}\text { Fuzzy } \\
\text { Multi-objective } \\
\text { using adaptive } \\
\text { Generic Algorithm }\end{array}$ & $\begin{array}{l}\text { Maximization of the Restored } \\
\text { Loads Considering the Load } \\
\text { Priorities and Minimization of the } \\
\text { Number of Switch Operations } \\
\text { Considering the Switch Priorities }\end{array}$ & $\sqrt{ }$ & & $\sqrt{ }$ & & & & & & & & & & & & & $\sqrt{ }$ & & Restoration & Software & {$[75]$} \\
\hline GA & $\begin{array}{l}\text { Maximizing the served load with } \\
\text { respect to load priorities after } \\
\text { fault occurrence }\end{array}$ & & & & & & $\sqrt{ }$ & & & & & & & & & & & $\sqrt{ }$ & Reconfiguration & Software & [76] \\
\hline $\begin{array}{c}\text { Real-time } \\
\text { heterogeneous MAS }\end{array}$ & $\begin{array}{l}\text { Maximize the energized loads in } \\
\text { the dc zonal system }\end{array}$ & $\sqrt{ }$ & & & $\sqrt{ }$ & $\sqrt{ }$ & & & & & & & & & & & & & $\begin{array}{l}\text { Normal and } \\
\text { pulse load }\end{array}$ & Software & [83] \\
\hline $\begin{array}{l}\text { Reconfiguration } \\
\text { algorithms }\end{array}$ & $\begin{array}{l}\text { Maximizing power delivery and } \\
\text { minimizing the number of } \\
\text { switching actions }\end{array}$ & $\sqrt{ }$ & & & & & & & & & & & & & & $\sqrt{ }$ & & $\sqrt{ } \quad \sqrt{ }$ & Reconfiguration & Software & [85] \\
\hline
\end{tabular}

* Refer to Appendix A. 
Maintaining reliable and secure communications is important for the operation of ship microgrids, especially with decentralized power management and control. Moreover, the communication between devices is time-critical and thus associated algorithms should be able to minimize delay and reduce computational complexity [86]. These requirements are very similar to those of the terrestrial microgrids and thus the communication methods developed for terrestrial microgrids can be extended for ship microgrids as well. An example of such a communication method is a security model based on message authentication code (MAC). This communication method is used for communication between terrestrial microgrid components including network, data, and attack models [87]. This model provides a secured communication environment with faster response and less memory compared with Rivest, Shamir, and Adleman (RSA), digital signature algorithm (DSA), and time valid hash one-time signature (TV-HORS) [88]. In islanded microgrids, low bandwidth communication is used to exchange information between a centralized controller and local controller in the secondary frequency microgrid. Delay margins in communication increase with the increase of gains of the secondary frequency controller which can be compensated by using a gain scheduling approach method [89]. The above-mentioned communication methods can be implemented in future ship microgrids due to their improved real-time response in order to ensure high performance and more reliability in ship microgrids.

Maintaining a low SFC is also another important objective of emerging ship PMS. Preplanned energy management by offline optimization algorithm can be used for fuel saving. However, in practical operation of ships, there are several innumerable contingencies, which influence the vessel operation. Therefore, using preplanned energy management will result in suboptimal fuel efficiency. In the other hand, the use of real-time energy management and optimization will provide more efficient fuel minimization [36,90]. Figure 9 shows a typical SFC curve for a marine diesel-engine [84]. The optimal fuel consumption can be achieved when the engine load is operated at the minimum SFC.

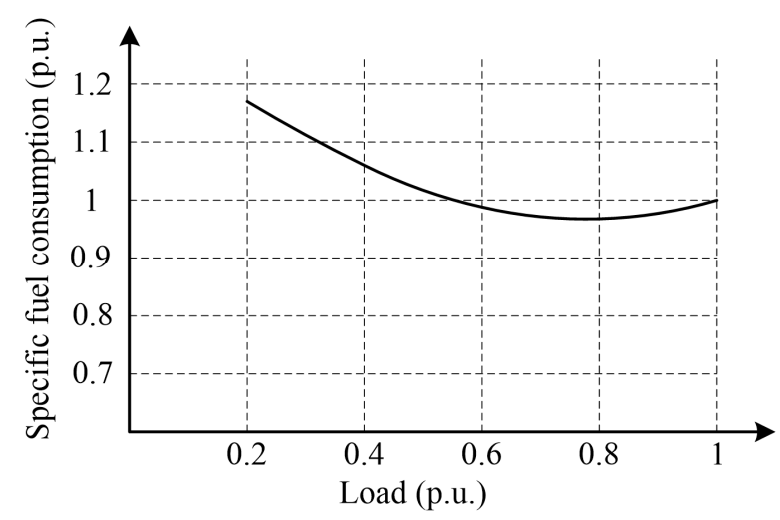

Figure 9. Typical specific fuel consumption curve of a marine diesel-engine.

Practically, the minimum SFC point does not represent the minimum fuel consumption of the engine due to power losses. Engine speed can also effect the SFC value as at high speeds it increases due to the increase in friction. At low speeds, it increases due to increased time for heat losses [91]. In addition, load ripples on the generator can cause ripples on the engine SFC. Therefore, fuel consumption can be optimized by minimizing SFC subjected to operational constraints such as engine speed and load ripple [84]. Energy storage systems can be used to absorb load ripples and thereby reduce SFC. This results in on-board emission reductions [66,92]. Additionally, the utilization of energy storage systems such as batteries is common to restore power system frequency and voltage [93,94]. Moreover, energy storage systems provide a reliable solution to supply multiple pulse loads $[95,96]$. 


\section{Concluding Remarks and Future Trends in Ship Microgrids}

With the growing demand for low emissions and fuel efficiency improvements in the maritime industry, alternative energy sources and energy storage technologies are becoming popular in ship microgrids. This paper presents a review on ship microgrid architectures, control technologies, and recent developments in power management strategies. In addition, the author's original research on the performance of droop control based power sharing is presented.

The growing interest for incorporating more-electric technologies into ships increases the demand for electrical power. Therefore, in large ships, HVAC distribution is preferred over LVAC distribution. Moreover, compared to radial architecture, ZEDS architectures are becoming popular in ship microgrids mainly due to their ability to prevent blackouts during faults in certain sections. Nowadays, the integration of renewable energy sources and energy storage systems is gaining attention due to the growing demand for emission reductions and fuel efficiency improvements. This trend is mainly supported by advancements in associated power electronics converter technologies. Moreover, energy storage systems are used to smoothen severe load transients and thereby obtain a more stable and secure shipboard power system. Hence, the ship microgrid can achieve power system stability by balancing demand and supply in real time while satisfying operational constraints. Moreover, with the advancements in power electronic technologies, the trend toward using DC distribution systems on-board is becoming popular [2]. One common recommended design is the medium voltage DC (MVDC) distribution system, with a voltage range of $1 \mathrm{kV}$ to $35 \mathrm{kV}$ [97]. This is mainly due to several advantages that DC distribution systems offer over AC distribution, including the possibility of implementing prime mover speed optimization to reduce fuel consumption and emissions, the flexibility to integrate renewable energy sources and energy storage systems, and the absence of reactive power and harmonic issues. Therefore, more focus is recommended to be taken on topics related to DC ship microgrids in future work.

On the control point of view, despite having certain limitations and disadvantages such as voltage and frequency deviations and the effects of current harmonics on the voltage regulation, droop control will continue to be the popular choice in AC ship microgrids. This is mainly due to the presence of synchronous generators and HV distribution systems in ships, a combination that is for droop control. Even though the presence of alternative energy technologies such as PV, wind, and fuel cells in ship microgrids continue to grow, their power levels are relatively small and thus the corresponding interfacing converters act as grid feeding inverters. Moreover, energy recovery technologies such as waste heat recovery are being incorporated into ship microgrids to improve fuel efficiency. The corresponding interfacing converters also work as grid feeding inverters. Once these grid feeding inverters are added into a ship microgrid, its control becomes complex. The hierarchical control framework, which is well explored in relation to complex terrestrial microgrids, can be adopted to these ship microgrids as well.

In terms of power management optimization, several studies reveal that using meta-heuristic optimization methods such as PSO and GA provide more promising optimization results than classical methods. This can be achieved, as meta-heuristic methods are capable of solving multi-objective optimization problems while satisfying several technical and operational constraints. Moreover, several studies used the MAS technique for the control and power management of shipmicrogrids. It is concluded from studies incorporating real-time control methods with MAS, that this combination provides better performance than using conventional MAS alone.

Communication plays a major role in assuring the safe and reliable operation of ship microgrids. In order to achieve these objectives, communication algorithms should be highly reliable, time-critical, and computationally not very complex. Terrestrial microgrids have similar requirements in terms of communications and thus similar methods, developed for terrestrial microgrids, can be adopted for ship microgrids. 
Author Contributions: M.D.A.A.-F., T.T. and S.G.J. wrote the paper; T.T. performed the experiments; H.E., Z.J. and J.M.G. provide guidance and critical review for the work.

Acknowledgments: This work was supported by the National Science Centre, Poland under Grant DEC2012/07/E/ST8/01688.

Conflicts of Interest: The authors declare no conflict of interest.

\section{Appendix A. Power Management Constraints}

\begin{tabular}{llll}
\hline VL & Voltage limit & F & Frequency \\
SC & Source capacity & PCC & Power Converter Capacity \\
PB & Power Balance & OT & Operating time of Gen. \\
GHG & Greenhouse gas emission & SS & Ship speed \\
TD & Travel distance & BP & Blackout Prevention \\
RR & Ramp Rates & GSS & Generator start/stop \\
ESS & Energy Storage System level (charge/discharge) & LL & Load level/Limit \\
SI & Stability index (transient angle stability index) & CC & Cable/branch current \\
PG & Power Generation limit & BC & Bus current \\
\hline
\end{tabular}

\section{References}

1. García-Olivares, A.; Solé, J.; Osychenko, O. Transportation in a 100\% renewable energy system. Energy Convers. Manag. 2018, 158, 266-285. [CrossRef]

2. Castellan, S.; Menis, R.; Tessarolo, A.; Luise, F.; Mazzuca, T. A review of power electronics equipment for all-electric ship MVDC power systems. Int. J. Electr. Power Energy Syst. 2018, 96, 306-323. [CrossRef]

3. Skjong, E.; Rødskar, E.; Molinas, M.; Johansen, T.; Cunningham, J. The marine vessel's electrical power system: From its birth to present day. Proc. IEEE 2015, 103, 2410-2424. [CrossRef]

4. Jayasinge, S.; Lokuketagoda, G.; Enshaei, H.; Shagar, V.; Ranmuthugala, D. Electro-technologies for energy efficiency improvement and low carbon emission in maritime transport. In Proceedings of the 16th Annual General Assembly of the International Association of Maritime Universities, Opatija, Croatia, 7-10 October 2015; pp. 119-123.

5. Sciberras, E.A.; Zahawi, B.; Atkinson, D.J. Reducing shipboard emissions-Assessment of the role of electrical technologies. Transp. Res. Part D Transp. Environ. 2017, 51, 227-239. [CrossRef]

6. Lan, H.; Dai, J.; Wen, S.; Hong, Y.-Y.; Yu, D.; Bai, Y. Optimal Tilt Angle of Photovoltaic Arrays and Economic Allocation of Energy Storage System on Large Oil Tanker Ship. Energies 2015, 8, 11515-11530. [CrossRef]

7. Han, J.; Charpentier, J.-F.; Tang, T. An Energy Management System of a Fuel Cell/Battery Hybrid Boat. Energies 2014, 7, 2799-2820. [CrossRef]

8. Geertsma, R.D.; Negenborn, R.R.; Visser, K.; Hopman, J.J. Design and control of hybrid power and propulsion systems for smart ships: A review of developments. Appl. Energy 2017, 194, 30-54. [CrossRef]

9. Andreasen, J.; Meroni, A.; Haglind, F. A Comparison of Organic and Steam Rankine Cycle Power Systems for Waste Heat Recovery on Large Ships. Energies 2017, 10, 547. [CrossRef]

10. Shagar, V.; Jayasinghe, S.G.; Enshaei, H. Effect of load changes on hybrid shipboard power systems and energy storage as a potential solution: A review. Inventions 2017, 2, 21. [CrossRef]

11. Vu, T.V.; Gonsoulin, D.; Diaz, F.; Edrington, C.S.; El-Mezyani, T. Predictive Control for Energy Management in Ship Power Systems Under High-Power Ramp Rate Loads. IEEE Trans. Energy Convers. 2017, 32, 788-797. [CrossRef]

12. Gonsoulin, D.; Vu, T.; Diaz, F.; Vahedi, H.; Perkins, D.; Edrington, C. Centralized MPC for Multiple Energy Storages in Ship Power Systems. In Proceedings of the IECON 2017-43rd Annual Conference of the IEEE Industrial Electronics Society, Beijing, China, 29 October-1 November 2017.

13. Gonsoulin, D.E.; Vu, T.V.; Diaz, F.; Vahedi, H.; Perkins, D.; Edrington, C.S. Coordinating Multiple Energy Storages Using MPC for Ship Power Systems. In Proceedings of the 2017 IEEE Electric Ship Technologies Symposium (ESTS), Arlington, VA, USA, 14-17 August 2017.

14. Crider, J.M.; Sudhoff, S.D. Reducing impact of pulsed power loads on microgrid power systems. IEEE Trans. Smart Grid 2010, 1, 270-277. [CrossRef] 
15. Kelley, J.P.; Wetz, D.A.; Reed, J.A.; Cohen, I.J.; Turner, G.K.; Lee, W.-J. The impact of power quality when high power pulsed DC and continuous AC loads are simultaneously operated on a MicroGrid testbed. In Proceedings of the 2013 IEEE Electric Ship Technologies Symposium (ESTS), Arlington, VA, USA, 22-24 April 2013; pp. 6-12.

16. Hebner, R.E.; Davey, K.; Herbst, J.; Hall, D.; Hahne, J.; Surls, D.D.; Ouroua, A. Dynamic load and storage integration. Proc. IEEE 2015, 103, 2344-2354. [CrossRef]

17. Hou, J.; Sun, J.; Hofmann, H. Control development and performance evaluation for battery/flywheel hybrid energy storage solutions to mitigate load fluctuations in all-electric ship propulsion systems. Appl. Energy 2018, 212, 919-930. [CrossRef]

18. McCoy, T.J. Electric Ships Past, Present, and Future [Technology Leaders]. IEEE Electrification Mag. 2015, 3, 4-11. [CrossRef]

19. Sudhoff, S.D.; Pekarek, S.D.; Swanson, R.R.; Duppalli, V.S.; Horvath, D.C.; Kasha, A.E.; Lin, R.; Marquet, B.D.; O'Regan, P.R.; Suryanarayana, H.; Yan, Y. A Reduced Scale Naval DC Microgrid to Support Electric Ship Research and Development. In Proceedings of the 2015 IEEE Electric Ship Technologies Symposium (ESTS), Alexandria, VA, USA, 21-24 June 2015; pp. 464-471.

20. Mashayekh, S.; Butler-Purry, K.L. An Integrated Security-Constrained Model-Based Dynamic Power Management Approach for Isolated Microgrids in All-Electric Ships. IEEE Trans. Power Syst. 2015, 30, 2934-2945. [CrossRef]

21. Elsayed, A.T.; Mohamed, A.A.; Mohammed, O.A. DC microgrids and distribution systems: An overview. Electr. Power Syst. Res. 2015, 119, 407-417. [CrossRef]

22. Al-Falahi, M.D.; Jayasinghe, S.; Enshaei, H. A review on recent size optimization methodologies for standalone solar and wind hybrid renewable energy system. Energy Convers. Manag. 2017, 143, 252-274. [CrossRef]

23. Al-Falahi, M.D.; Nimma, K.S.; Jayasinghe, S.; Enshaei, H. Sizing and modeling of a standalone hybrid renewable energy system. In Proceedings of the IEEE Annual Southern Power Electronics Conference (SPEC), Auckland, New Zealand, 5-8 December 2016; pp. 1-6.

24. Jayasinghe, S.G.; Meegahapola, L.; Fernando, N.; Jin, Z.; Guerrero, J.M. Review of ship microgrids: System architectures, storage technologies and power quality aspects. Inventions 2017, 2, 4. [CrossRef]

25. Jin, Z.; Savaghebi, M.; Vasquez, J.C.; Meng, L.; Guerrero, J.M. Maritime DC Microgrids-A Combination of Microgrid Technologies and Maritime Onboard Power System for Future Ships. In Proceedings of the 2016 8th International Power Electronics and Motion Control Conference-Ecce Asia (IPEMC 2016-ECCE Asia), Hefei, China, 22-26 May 2016.

26. Guerrero, J.M.; Vasquez, J.C.; Matas, J.; De Vicuña, L.G.; Castilla, M. Hierarchical control of droop-controlled $\mathrm{AC}$ and DC microgrids-A general approach toward standardization. IEEE Trans. Ind. Electron. 2011, 58, 158-172. [CrossRef]

27. Liang, J.; Qi, L.; Lindtjørn, J.O.; Wendt, F. Frequency Dependent DC Voltage Droop Control for Hybrid Energy Storage in DC Microgrids. In Proceedings of the 2015 IEEE Power \& Energy Society General Meeting, Denver, CO, USA, 26-30 July 2015; pp. 1-5.

28. Farasat, M.; Arabali, A.S.; Trzynadlowski, A.M. A novel control principle for all-electric ship power systems. In Proceedings of the 2013 IEEE Electric Ship Technologies Symposium (ESTS), Arlington, VA, USA, 22-24 April 2013; pp. 178-184.

29. Shang, C.; Srinivasan, D.; Reindl, T. Economic and Environmental Generation and Voyage Scheduling of All-Electric Ships. IEEE Trans. Power Syst. 2015, 31, 4087-4096. [CrossRef]

30. Nasri, M.; Hossain, M.R.; Ginn, H.L.; Moallem, M. Agent-based real-time coordination of power converters in a DC shipboard power system. In Proceedings of the 2015 IEEE Electric Ship Technologies Symposium (ESTS), Alexandria, VA, USA, 21-24 June 2015; pp. 8-13.

31. Paran, S.; Vu, T.; El Mezyani, T.; Edrington, C. MPC-based power management in the shipboard power system. In Proceedings of the 2015 IEEE Electric Ship Technologies Symposium (ESTS), Alexandria, VA, USA, 21-24 June 2015; pp. 14-18.

32. Tang, D.; Yan, X.; Yuan, Y.; Wang, K.; Qiu, L. Multi-agent Based Power and Energy Management System for Hybrid Ships. In Proceedings of the 2015 International Conference on Renewable Energy Research and Applications (ICRERA), Palermo, Italy, 22-25 November 2015; pp. 383-387. 
33. Shagar, V.; Jayasinghe, S.; Enshaei, H. Frequency Transient Suppression in Hybrid Electric Ship Power Systems: A Model Predictive Control Strategy for Converter Control with Energy Storage. Inventions 2018, 3, 13. [CrossRef]

34. Pish, S.; Herbst, J.; Wardell, D.; Gattozzi, A.; Flynn, M. Power management and energy storage experiments on a MW-scale naval power system test-bed. In Proceedings of the 2015 IEEE Electric Ship Technologies Symposium (ESTS), Alexandria, VA, USA, 21-24 June 2015; pp. 453-458.

35. Rose, M.W.; Cuzner, R.M. Fault isolation and reconfiguration in a three-zone system. In Proceedings of the 2015 IEEE Electric Ship Technologies Symposium (ESTS), Alexandria, VA, USA, 21-24 June 2015; pp. $409-414$.

36. Jin, Z.; Sulligoi, G.; Cuzner, R.; Meng, L.; Vasquez, J.C.; Guerrero, J.M. Next-Generation Shipboard DC Power System: Introduction Smart Grid and dc Microgrid Technologies into Maritime Electrical Netowrks. IEEE Electrification Mag. 2016, 4, 45-57. [CrossRef]

37. Huang, K.; Srivastava, S.K.; Cartes, D.A.; Sun, L.-H. Market-based multiagent system for reconfiguration of shipboard power systems. Electr. Power Syst. Res. 2009, 79, 550-556. [CrossRef]

38. Hall, D.T. Practical Marine Electrical Knowladge, 3rd ed.; Witherby Seamanship: Livingston, UK, 2014.

39. Jin, Z.; Meng, L.; Guerrero, J.M.; Han, R. Hierarchical Control Design for a Shipboard Power System With DC Distribution and Energy Storage Aboard Future More-Electric Ships. IEEE Trans. Ind. Inform. 2018, 14, 703-714. [CrossRef]

40. Hegner, H.; Desai, B. Integrated fight through power. In Proceedings of the 2002 IEEE Power Engineering Society Summer Meeting, Chicago, IL, USA, 21-25 July 2002; pp. 336-339.

41. Khushalani, S.; Solanki, J.; Schulz, N. Optimized restoration of combined ac/dc shipboard power systems including distributed generation and islanding techniques. Electr. Power Syst. Res. 2008, 78, 1528-1536. [CrossRef]

42. IEEE Std 45.3 ${ }^{\mathrm{TM}}$-2015. IEEE Recommended Practice for Shipboard Electrical Installations—Systems Engineering; IEEE: Piscataway, NJ, USA, 2015.

43. Nelson, J.P.; Burns, D.; Seitz, R.; Leoni, A. The grounding of marine power systems: Problems and solutions. In Proceedings of the 2004 Fifty-First Annual Conference Petroleum and Chemical Industry Technical Conference, San Francisco, CA, USA, 13-15 September 2004; pp. 151-161.

44. Papadimitriou, C.; Zountouridou, E.; Hatziargyriou, N. Review of hierarchical control in DC microgrids. Electr. Power Syst. Res. 2015, 122, 159-167. [CrossRef]

45. Cosse, R.E.; Alford, M.D.; Hajiaghajani, M.; Hamilton, E.R. Turbine/generator governor droop/isochronous fundamentals-A graphical approach. In Proceedings of the 2011 Record of Conference Papers Industry Applications Society 58th Annual IEEE Petroleum and Chemical Industry Conference (PCIC), Toronto, ON, Canada, 19-21 September 2011; pp. 1-8.

46. Olson, G. Paralleling Dissimilar Generators: Part 3-Load Sharing Compatibility. In White Paper; Cummins Power Generation: Ramsgate, UK, 2010.

47. Johannessen, P.F.; Mathiesen, E. Advanced Failure Detection and Handling in Power Management System. In Proceedings of the Dynamic Positioning Committee, Kongsberg, Norway, 13-14 October 2009.

48. Rocabert, J.; Luna, A.; Blaabjerg, F.; Rodr, P. Control of Power Converters in AC Microgrids. IEEE Trans. Power Electron. 2012, 27, 4734-4749. [CrossRef]

49. DNV-GL. Rules for Classification. Ships. Part 4 Systems and Components; Chapter 8 Electrical Installations; DNV-GL: Oslo, Norway, 2016.

50. International Naval Ships. Part 4 Vessel Systems and Machinery. In Guide for Building and Classing; ABS: Houston, TX, USA, 2016.

51. Register, L.S. Rules and Regulations for Classification of Ships; Lloyd's Register: London, UK, 2016.

52. Vu, T.V.; Perkins, D.; Diaz, F.; Gonsoulin, D.; Edrington, C.S.; El-Mezyani, T. Robust adaptive droop control for DC microgrids. Electr. Power Syst. Res. 2017, 146, 95-106. [CrossRef]

53. Han, H.; Hou, X.; Yang, J.; Wu, J.; Su, M.; Guerrero, J.M. Review of Power Sharing Control Strategies for Islanding Operation of AC Microgrids. IEEE Trans. Smart Grid 2016, 7, 200-215. [CrossRef]

54. Eid, B.M.; Rahim, N.A.; Selvaraj, J.; Khateb, A.H.E. Control Methods and Objectives for Electronically Coupled Distributed Energy Resources in Microgrids: A Review. IEEE Syst. J. 2016, 10, 446-458. [CrossRef]

55. Olivares, D.E.; Mehrizi-Sani, A.; Etemadi, A.H.; Ca, C.A.; Iravani, R.; Kazerani, M.; Hajimiragha, A.H.; Gomis-Bellmunt, O.; Saeedifard, M.; Palma-Behnke, R.; et al. Trends in Microgrid Control. IEEE Trans. Smart Grid 2014, 5, 1905-1919. [CrossRef] 
56. Tarasiuk, T.; Pilat, A.; Szweda, M. Experimental Study on Impact of Ship Electric Power Plant Configuration on Power Quality in the Ship Power System. In Proceedings of the Proceedings of the World Congress on Engineering, London, UK, 2-4 July 2014.

57. Liu, Y.; Zhang, Q.; Wang, C.; Wang, N. A control strategy for microgrid inverters based on adaptive three-order sliding mode and optimized droop controls. Electr. Power Syst. Res. 2014, 117, 192-201. [CrossRef]

58. Cárdenas, R.; Juri, C.; Pena, R.; Wheeler, P.; Clare, J. The Application of Resonant Controllers to Four-Leg Matrix Converters Feeding Unbalanced or Nonlinear Loads. IEEE Trans. Power Electron. 2012, 27, 1120-1129. [CrossRef]

59. Blaabjerg, F.; Teodorescu, R.; Liserre, M.; Timbus, A.V. Overview of Control and Grid Synchronization for Distributed Power Generation Systems. IEEE Trans. Ind. Electron. 2006, 53, 1398-1409. [CrossRef]

60. Jayasinghe, S.G.; Al-Falahi, M.; Enshaei, H.; Fernando, N.; Tashakori, A. Floating power platforms for mobile cold-ironing. In Proceedings of the IEEE Annual Southern Power Electronics Conference (SPEC), Auckland, New Zealand, 5-8 December 2016; pp. 1-5.

61. Palma-Behnke, R.; Benavides, C.; Lanas, F.; Severino, B.; Reyes, L.; Llanos, J.; Sáez, D. A microgrid energy management system based on the rolling horizon strategy. IEEE Trans. Smart Grid 2013, 4, 996-1006. [CrossRef]

62. Meng, L.; Sanseverino, E.R.; Luna, A.; Dragicevic, T.; Vasquez, J.C.; Guerrero, J.M. Microgrid supervisory controllers and energy management systems: A literature review. Renew. Sustain. Energy Rev. 2016, 60, 1263-1273. [CrossRef]

63. Feng, X.; Butler-Purry, K.L.; Zourntos, T. A multi-agent system framework for real-time electric load management in MVAC all-electric ship power systems. IEEE Trans. Power Syst. 2015, 30, 1327-1336. [CrossRef]

64. Feng, X.; Butler-Purry, K.L.; Zourntos, T. Multi-agent system-based real-time load management for all-electric ship power systems in DC zone level. IEEE Trans. Power Syst. 2012, 27, 1719-1728. [CrossRef]

65. Di Silvestre, M.L.; Graells, M.; Guerrero, J.M.; Luna, A.C.; Mineo, L.; Nguyen, N.; Sanseverino, E.R.; Vasquez, J.C. Energy Management Systems and tertiary regulation in hierarchical control architectures for islanded micro-grids. In Proceedings of the 2015 15th International Conference on Environment and Electrical Engineering (EEEIC), Rome, Italy, 10-13 June 2015.

66. Hebner, R.E.; Uriarte, F.M.; Kwasinski, A.; Gattozzi, A.L.; Estes, H.B.; Anwar, A.; Cairoli, P.; Dougal, R.A.; Feng, X.; Chou, H.-M.; et al. Technical cross-fertilization between terrestrial microgrids and ship power systems. J. Mod. Power Syst. Clean Energy 2015, 4, 161-179. [CrossRef]

67. Debs, A.S. Modern Power Systems Control and Operation; Springer Science \& Business Media: Berlin, Germany, 2012.

68. Kanellos, F.D.; Tsekouras, G.J.; Hatziargyriou, N.D. Optimal demand-side management and power generation scheduling in an all-electric ship. IEEE Trans. Sustain. Energy 2014, 5, 1166-1175. [CrossRef]

69. Kanellos, F. Optimal power management with GHG emissions limitation in all-electric ship power systems comprising energy storage systems. IEEE Trans. Power Syst. 2014, 29, 330-339. [CrossRef]

70. Kanellos, F.D.; Prousalidis, J.M.; Tsekouras, G.J. Control system for fuel consumption minimization-gas emission limitation of full electric propulsion ship power systems. Proc. Inst. Mech. Eng. Part M 2014, 228, 17-28. [CrossRef]

71. Nimma, K.; Al-Falahi, M.; Nguyen, H.D.; Jayasinghe, S.D.G.; Mahmoud, T.; Negnevitsky, M. Grey Wolf Optimization-Based Optimum Energy-Management and Battery-Sizing Method for Grid-Connected Microgrids. Energies 2018, 11, 847. [CrossRef]

72. Hou, J.; Sun, J.; Hofmann, H. Adaptive model predictive control with propulsion load estimation and prediction for all-electric ship energy management. Energy 2018, 150, 877-889. [CrossRef]

73. Hou, J.; Sun, J.; Hofmann, H.F. Mitigating Power Fluctuations in Electric Ship Propulsion With Hybrid Energy Storage System: Design and Analysis. IEEE J. Ocean. Eng. 2018, 43, 93-107. [CrossRef]

74. Kanellos, F.D.; Anvari-Moghaddam, A.; Guerrero, J.M. A cost-effective and emission-aware power management system for ships with integrated full electric propulsion. Electr. Power Syst. Res. 2017, 150, 63-75. [CrossRef]

75. Jiang, Y.; Jiang, J.; Zhang, Y. A novel fuzzy multiobjective model using adaptive genetic algorithm based on cloud theory for service restoration of shipboard power systems. IEEE Trans. Power Syst. 2012, 27, 612-620. [CrossRef]

76. Shariatzadeh, F.; Vellaithurai, C.B.; Biswas, S.S.; Zamora, R.; Srivastava, A.K. Real-time implementation of intelligent reconfiguration algorithm for microgrid. IEEE Trans. Sustain. Energy 2014, 5, 598-607. [CrossRef] 
77. Reichel, M.; Minchev, A.; Larsen, N.L. Trim Optimisation-Theory and Practice. TransNav Int. J. Mar. Navig. Saf. Sea Transp. 2014, 8, 387-392. [CrossRef]

78. Kankanala, P.; Srivastava, S.C.; Srivastava, A.K.; Schulz, N.N. Optimal control of voltage and power in a multi-zonal MVDC shipboard power system. IEEE Trans. Power Syst. 2012, 27, 642-650. [CrossRef]

79. Vu, T.V.; Paran, S.; Mezyani, T.E.; Edrington, C.S. Real-time Distributed Power Optimization in the DC Microgrids of Shipboard Power Systems. In Proceedings of the 2015 IEEE Electric Ship Technologies Symposium (ESTS), Alexandria, VA, USA, 21-24 June 2015.

80. Logenthiran, T.; Srinivasan, D.; Khambadkone, A.M. Multi-agent system for energy resource scheduling of integrated microgrids in a distributed system. Electr. Power Syst. Res. 2011, 81, 138-148. [CrossRef]

81. Dou, C.; Yue, D.; Guerrero, J.M.; Xie, X.; Hu, S. Multiagent System-Based Distributed Coordinated Control for Radial DC Microgrid Considering Transmission Time Delays. IEEE Trans. Smart Grid 2017, 8, 2370-2381. [CrossRef]

82. Zhao, B.; Xue, M.; Zhang, X.; Wang, C.; Zhao, J. An MAS based energy management system for a stand-alone microgrid at high altitude. Appl. Energy 2015, 143, 251-261. [CrossRef]

83. Feng, X.; Butler-Purry, K.L.; Zourntos, T. Real-time electric load management for DC zonal all-electric ship power systems. Electr. Power Syst. Res. 2018, 154, 503-514. [CrossRef]

84. Zahedi, B.; Norum, L.E.; Ludvigsen, K.B. Optimized efficiency of all-electric ships by dc hybrid power systems. J. Power Sources 2014, 255, 341-354. [CrossRef]

85. Bose, S.; Pal, S.; Natarajan, B.; Scoglio, C.M.; Das, S.; Schulz, N.N. Analysis of Optimal Reconfiguration of Shipboard Power Systems. IEEE Trans. Power Syst. 2012, 27, 189-197. [CrossRef]

86. Nimma, K.; Faraj, S. Modeling Intelligent Control Switch IEC 61850 Based Substation Automation Communication. Appl. Syst. Innov. 2018, 1, 7. [CrossRef]

87. Kounev, V.; Tipper, D.; Yavuz, A.A.; Grainger, B.M.; Reed, G.F. A Secure Communication Architecture for Distributed Microgrid Control. IEEE Trans. Smart Grid 2015, 6, 2484-2492. [CrossRef]

88. Hou, C.; Jiang, H.; Yang, Y.; Rui, W.; Hu, L. Research on Implementing Real Time Ethernet for Ship Power System. In Proceedings of the 2010 2nd International Workshop on Intelligent Systems and Applications (ISA), Wuhan, China, 22-23 May 2010; pp. 1-4.

89. Liu, S.; Wang, X.; Liu, P.X. Impact of communication delays on secondary frequency control in an islanded microgrid. IEEE Trans. Ind. Electron. 2015, 62, 2021-2031. [CrossRef]

90. EMMA Addvisory Suite. The Complete, East-to-Use Energy Management Solution-Including Monitoring and Optmization; ABB: Zürich, Switzerland, 2012; pp. 1-4.

91. Altosole, M.; Benvenuto, G.; Campora, U.; Laviola, M.; Trucco, A. Waste Heat Recovery from Marine Gas Turbines and Diesel Engines. Energies 2017, 10, 718. [CrossRef]

92. Tichavska, M.; Tovar, B.; Gritsenko, D.; Johansson, L.; Jalkanen, J.P. Air emissions from ships in port: Does regulation make a difference? Transp. Policy 2017. [CrossRef]

93. Koller, M.; Borsche, T.; Ulbig, A.; Andersson, G. Review of grid applications with the Zurich 1MW battery energy storage system. Electr. Power Syst. Res. 2015, 120, 128-135. [CrossRef]

94. Singh, M.; Lopes, L.A.; Ninad, N.A. Grid forming Battery Energy Storage System (BESS) for a highly unbalanced hybrid mini-grid. Electr. Power Syst. Res. 2015, 127, 126-133. [CrossRef]

95. Misyris, G.S.; Marinopoulos, A.; Doukas, D.I.; Tengnér, T.; Labridis, D.P. On battery state estimation algorithms for electric ship applications. Electr. Power Syst. Res. 2017, 151, 115-124. [CrossRef]

96. Lashway, C.R.; Elsayed, A.T.; Mohammed, O.A. Hybrid energy storage management in ship power systems with multiple pulsed loads. Electr. Power Syst. Res. 2016, 141, 50-62. [CrossRef]

97. IEEE Standard 1709-2010. IEEE Recommended Practice for $1 \mathrm{kV}$ to $35 \mathrm{kV}$ Medium-Voltage DC Power Systems on Ships; IEEE: Piscataway, NJ, USA, 2010.

(C) 2018 by the authors. Licensee MDPI, Basel, Switzerland. This article is an open access article distributed under the terms and conditions of the Creative Commons Attribution (CC BY) license (http:/ / creativecommons.org/licenses/by/4.0/). 


\section{Chapter 3: \\ Comparison of Several Operational Scenarios of Electric Ferries}

This chapter provides design and simulation of power systems for short-haul electric ferries. In addition, the affecting parameters of the sizing of battery for short-haul ferries are discussed. This chapter consists of two subchapters.

Part A: Comparison between Hybrid and Fully Electric Ferries in terms of Operational Cost and Battery Lifetime

Part B: Techno-Economic Feasibility Study of Battery-Powered Ferries 


\section{Chapter 3}

\subsection{Part A: Comparison Between Hybrid and Fully Electric Ferries Based on Operational Cost and Battery Lifetime}

This subchapter presents design and simulation of a classical rule-based power management method to optimally manage the power system of a short-haul ferry for achieving minimum operating cost. Moreover, an approach to find the optimal battery capacity is presented. The proposed methods are applied for three configurations, each of which is assessed using the operating cost factor. This subchapter has been accepted for publication in the 2019 9th International Conference on Power and Energy Systems (ICPES). The citation of the article is M. D. A. Al-Falahi, S. G. Jayasinghe, H. Enshaei, C. Baguley, and U. Madawala, "Diesel, Hybrid or Fully Electric Ferry, A Method For Operating Cost Analysis And Battery Size Optimization," 2019 9th International Conference on Power and Energy Systems (ICPES). Accepted, 2019..

\section{Chapter 3A has been removed for copyright or proprietary reasons.}




\section{Chapter 3}

\subsection{Part B: Techno-Economic Feasibility Study of Battery- Powered Ferries}

This subchapter presents further investigation on all-electric battery-powered ferries by providing techno-economic analysis. The technical study includes sizing of the battery storage system based on DoD and maximum load scenario. The economic study includes initial investment, operational cost, and maintenance cost of the battery-powered electric ferry and regards the PBP and battery lifecycle as assessment factors. This subchapter has been published in the Proceeding of the 2018 IEEE 4th Southern Power Electronics Conference (SPEC 2018). The citation of the article is

Al-Falahi, M. D., Coleiro, J., Jayasinghe, S. D. G., Enshaei, H., Garaniya, V., Baguley, C., \& Madawala, U. (2018, December). Techno-Economic Feasibility Study of Battery-Powered Ferries. In 2018 IEEE 4th Southern Power Electronics Conference (SPEC) (pp. 1-7). IEEE. 


\title{
Techno-Economic Feasibility Study of Battery- Powered Ferries
}

\author{
${ }^{1}$ Monaaf D.A. Al-Falahi*, ${ }^{1}$ James Coleiro, ${ }^{1}$ S. D. G. Jayasinghe, ${ }^{1}$ Hossein Enshaei, ${ }^{1}$ Vikram Garaniya, ${ }^{2}$ Craig. Baguley and \\ ${ }^{3}$ Udaya. Madawala \\ ${ }^{1}$ Australian Maritime College, College of Sciences and Engineering, University of Tasmania, Newnham, Australia \\ ${ }^{2}$ Auckland University of Technology, Auckland, New Zealand \\ ${ }^{3}$ The University of Auckland, Auckland, New Zealand \\ e-mail:*monaaf.alfalahi@utas.edu.au; jcoleiro@utas.edu.au; shanthaj@utas.edu.au; hossein.enshaei@utas.edu.au; \\ v.garaniya@utas.edu.au; craig.baguley@aut.ac.nz; u.madawala@auckland.ac.nz
}

\begin{abstract}
The move towards incorporating more-electric solutions in the transportation sector has gained increased momentum during the last decade. Cost of fossil fuels and environmental impacts of emissions are the main driving factors behind this growing trend. Nevertheless, advancements in battery technologies are the key enablers for the widespread application of electric alternatives in a more realistic manner. This paper looks into this trend from electric ferries perspective and presents a technical and economic feasibility assessment. The technical study includes sizing of the battery storage system based on Depth-ofDischarge (DOD) and maximum load scenario. The proposed sizing is validated against the measured load profile. The economic study includes initial investment, operational cost and maintenance cost of a battery powered electric ferry. The economic analysis considered the payback period (PBP) and battery lifecycles as assessment factors. The technical assessment results revealed that the proposed battery system can efficiently power the ferry within the stipulated DOD range. The maximum DOD achieved is $70 \%$, which provided a reasonable lifetime of 10.7 years. The economic analysis revealed that the battery's DOD has significant effects on the investment cost of the system and the PBP. The PBP is found to be 6.7 years which is $37 \%$ less than the lifetime of the battery. Overall, the battery-powered ferry is found to be feasible with $51.3 \%$ operational costs saving compared to the diesel-electric alternative.
\end{abstract}

Keywords- Battery powered ferry, diesel electric ferry, energy storage system, payback period.

\section{INTRODUCTION}

$\mathrm{W}$ ITH the aim of reducing the dependency on fossil fuels and harmful effects of their emissions, environmentally friendly transportation methods are extensively being explored in relation to land transportation with promising results [1]-[3]. Following this trend, many marine organizations and classification societies have also identified that the electrification of marine vessels is a promising approach to limit ship emissions [4]-[6].

Ship electrification is certainly not a new concept and it has been recognized for decades as a potential technological advancement for reducing emissions [7]. Nevertheless, energy storage has been and still continue to be the limiting factor for large ships [8]. This, however, is changing in the automobile industry with most of the major car manufacturing companies offering electric vehicles with acceptable, if not comparable, power and range from a single charge [9]. The successful implementation of batteries in automotive industry provide an insight for marine industry to explore and implement such storage technologies for ships [8].
Recent studies have indicated that the implementation of battery storage technologies in marine industry can be technically and economically feasible [10], [11]. However, for large marine vessels, batteries are used only as back-up power supplies or for peak demand shaving [10]. On the other hand, batteries can be used as a main power supply for small size marine vessels such as passenger boats and domestic short haul ferries [5], [12]. Moreover, as most of the domestic ferries have fixed routes, on-board batteries can be charged from the shore power supply during passenger/vehicle transfer. The Norwegian company, Norled AS, recently completed the construction of its all battery powered ferry 'MF Ampere' with 50\% reduction in energy usage compared to fossil fuel alternative [5]. Following this successful implementation, plans have been revealed introduce more battery powered ferries in the future [5].

Many studies are currently taking place and looking deeply into understand whether the batteries and associated technologies have advanced to a point where companies can profit by pursuing totally electric alternatives. So far, battery powered propulsion has only been briefly looked into within the maritime industry to oppose the typical diesel/petrol propulsion systems but halted, mainly because batteries have lacked in storage capacity, range and power generation. The fewer options which have existed prior to the $21^{\text {st }}$ century have also been relatively costly due to the sluggish rate of technological advancement and the conservative nature of the maritime industry [13].

Technical assessments of all-electric short haul ferries have been reported in several studies. Fuel cell powered domestic ferry [14], fuel cell powered tourist boat [15], fuel cell-battery powered boat [16], supercapacitor plug-in ferry [17] and battery-diesel genset hybrid passenger ferry are examples for such studies. These studies have not extensively investigated the effect of onboard battery packs size and DoD on the lifetime of the battery and PBP of the system. Therefore, the aim of this paper is to investigate the technical and economic feasibility of converting a dieselpowered ferry to a battery-powered electric ferry. The case study uses a diesel electric ferry which is explained in detail in section II. Sizing of the proposed system is presented in section III. Economic factors and cost analysis are presented 
in section IV. Simulation results and the economic analysis results are discussed in section V. Conclusions drawn from the results of the study are presented in section VI.

\section{CASE STUDY: BRUNY ISLAND FERRY}

Bruny Island is located off the south-eastern coast of Tasmania, Australia and encompasses approximately 363 square kilometres. The island is increasingly becoming a popular tourist destination for Tasmanian locals and visitors alike. Reliable and efficient access to the Island is thus increasingly important and predominately achieved through a ferry service which operates between Kettering (Hobart) and Roberts Point (Bruny Island) with the route represented in Fig.1. Two ferry services are provided in this route Mirambeena and MV Bowen. In this paper, MV Bowen ferry is used as a case study.

MV Bowen is a barge that was introduced to service in early 2015. The ferry performs seven trips per day for six days a week. The ferry's existing power system is diesel electric with two gen-sets and diagonally opposed azimuth thrusters in the forward and aft sections. Gen-sets provide power to the service loads as well. More details of the ferry are given in Table 1.

The load profile of Bowen ferry, measured for one round trip, is shown in Fig. 2. The high-power demand occurs during cruising. In such short haul ferries, most of the power is used to drive propulsion motors.

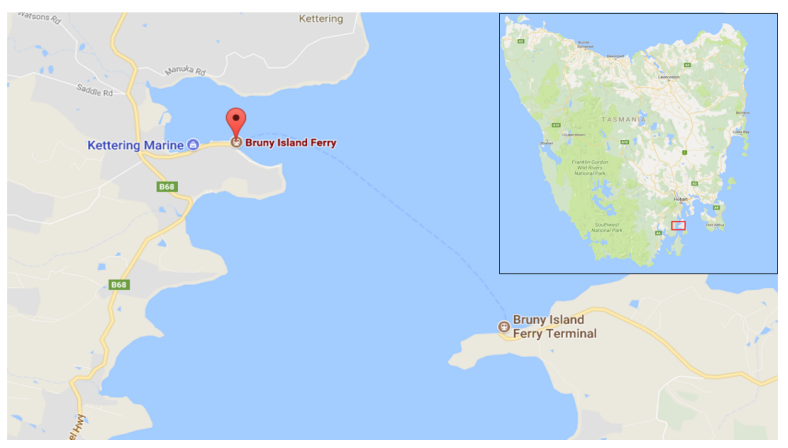

Fig. 1. Bruny Island ferry route

Table 1. Ferry specifications

\begin{tabular}{|l|c|}
\hline Powering & $2 \times 400 \mathrm{kVA}$ gen sets \\
\hline Travel distance & $6.2 \mathrm{~km}$ (round - trip) \\
\hline Fuel type & Diesel \\
\hline Travel duration & 60 minutes (round trip) \\
\hline
\end{tabular}

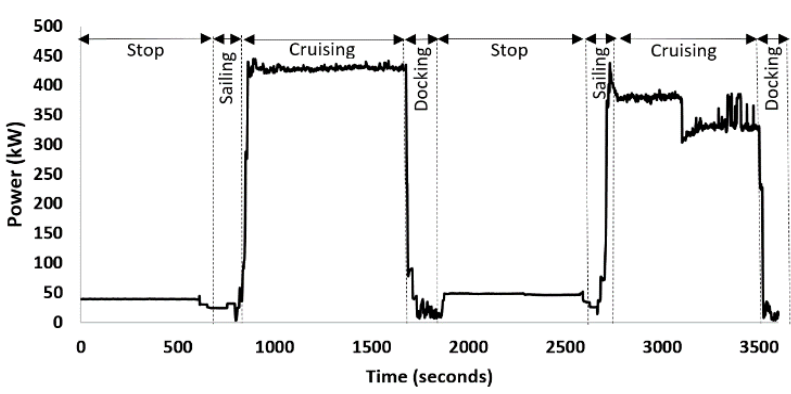

Fig. 2. Measured load profile of Bowen ferry

\section{PROPOSED SYSTEM SIZING}

\section{A. Proposed power architecture}

The proposed power system is shown in Fig. 3. A DC distribution system is used. This is since the main power sources are battery banks. DC/DC converters are used to regulate the voltage at the common $\mathrm{DC}$ bus. The shore charging station utilizes the readily available $11 \mathrm{kV}, 3$-phase supply from the utility grid. Two rectifiers are fed by a threephase transformer. The rectifiers convert AC voltages to DC voltage which is then used to charge the onboard battery packs via a shore connection cable and plug assembly.

\section{B. Battery system}

The sizing of the battery is determined based on two constraints: Depth of Discharge (DOD) and maximum load scenario. The impact of the battery weight is not considered as it is relatively small compared to the total load capacity of the ferry.

Based on Fig. 2, the total energy required for a single return trip found to be $200.17 \mathrm{kWh}$. According to marine battery manufacturers, the DOD of the battery should not exceed $80 \%$ to ensure longer lifetime of the battery modules [18]-[20]. Therefore, the selected maximum DOD of the battery pack is $80 \%$.

The maximum load demand while cruising is occurred in the first cruising period. The maximum energy consumption at berth occurred at Terminal 2. Therefore, in order to find the battery capacity, the maximum load demand of the first cruising is used for the second cruising period as well. In addition, the maximum load demand occurred at the second terminal is used for the first terminal as well. Based on this modified load scenario, the capacity of the battery pack can be calculated as:

$$
\begin{aligned}
E_{B} & =\frac{N_{S}\left[E_{T}^{\text {max }}+E_{C}^{\text {max }}\right]+0.2\left[E_{T}^{\text {max }}+E_{C}^{\text {max }}\right]}{\eta_{d}} \\
E_{B} & =\frac{1.2 N_{S}\left[E_{T}^{\text {max }}+E_{C}^{\text {max }}\right]}{\eta_{d}}
\end{aligned}
$$

Where $E_{B}$ is the energy capacity of the battery pack in kWh, $N_{S}$ is the number of stops per one round-trip and $E_{T}^{\max }$ is the highest energy at one terminal in $\mathrm{kWh}, E_{C}^{\max }$ is the highest energy while the ferry is cruising, the constant 0.2 represents

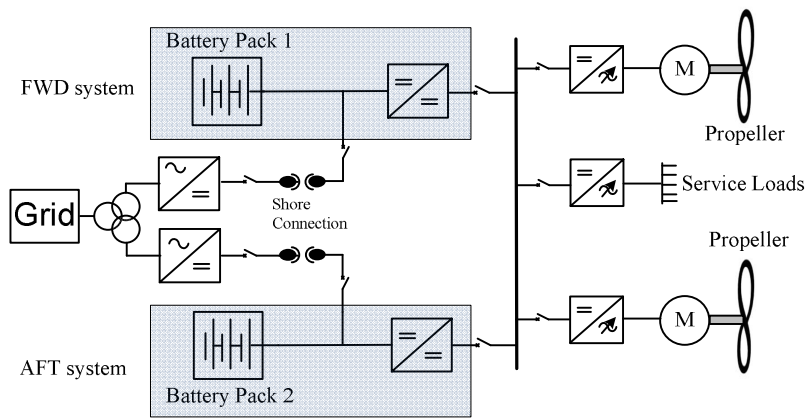

Fig. 3. Proposed system power architecture 
the minimum state of charge (SOC) of the battery $(80 \%$ DOD) recommended by several marine battery manufacturers to maintain a reasonable cycle life and $\eta_{d}$ the discharging efficiency of the battery.

Based on Eq. 2, the required capacity of the battery energy storage system found to be $273.4 \mathrm{kWh}$. The battery storage is divided into two packs: one installed at the port side FWD system) of the ferry and the other is installed at the starboard (AFT system) of the ferry. As a result, the capacity of each battery pack is $136.7 \mathrm{kWh}$.

The technical specifications of the lithium-ion battery module are taken from PBES battery manufacturer [18]. The summary of the battery module specifications is shown in Table 2.

Based on this module specifications, the most suitable combination of the module in each pack is shown in Fig. 4 and the specifications of each battery pack is provided in Table 3.

\section{ECONOMIC ELEMENTS}

Economic assessment for the electrification of MV Bowen is completed through utilization of cost estimations and actual quotes for associated elements. The elements are initial investment costs, operational costs, battery lifetime and payback period.

\section{A. Initial Costs}

Initial investment cost includes the cost of battery packs and shore charging station. The cost of the battery pack is determined based on the capacity given in Table 3 . The price of lithium nickel manganese cobalt oxide (NMC) batteries that are suitable for marine applications is considered as $\$ 1000$ per $\mathrm{kWh}[21]$.

Infrastructure and installation costs are identified through multiple quotes received from potential suppliers and contractors where an averaged value was taken. Quotations included all relevant systems and parts which are necessary to keep the vessels electric system operating (excluding the battery packs). This includes cables required to connect to the local electricity grid, data gateway and transformer unit. The system is designed to have capabilities of connecting to the local grid at Kettering and provide sufficient power to recharge the vessel within 11 minutes (instantaneous power requirement of 1.4 MW).

\section{B. Operational Cost}

The operational cost includes the cost of energy required to charge the onboard battery packs. Yearly recharging costs are determined with electricity prices provided by Aurora Energy (Time of Use (ToU) -Tariff 94) [22]. Table 4 shows the energy prices for different periods.

It is assumed that the vessel is recharged after each of its seven trips per day with its final recharge occurring overnight (off-peak period) to ensure costs are reduced.
Furthermore, it is assumed that the vessel would operate year-round (excluding Sundays) to represent a more realistic and sensible economic situation. The recharging profile for the vessel is shown in Table 5.

The ferry is operated for 259 weekdays and 53 weekend days (excluding Sundays) a year. The total recharging costs per year based on the operational profile using the following equation:

$$
C_{c h}=D_{\text {weekday }} C_{\text {weekday }}+D_{\text {weeked }} C_{\text {weekend }}
$$

where $C_{c h}$ is the annual charging costs (\$AUD), $C_{\text {weekday }}$ is the weekdays cost per week (\$AUD), $C_{\text {weekend }}$ is the weekend cost per week (\$AUD) $D_{\text {weekday }}$ is the number of operating weekdays in a year and $D_{\text {weekend }}$ is the number of operating weekend days in a year.

$C_{\text {weekday }}$ and $C_{\text {weekend }}$ can be calculated by the following equations:

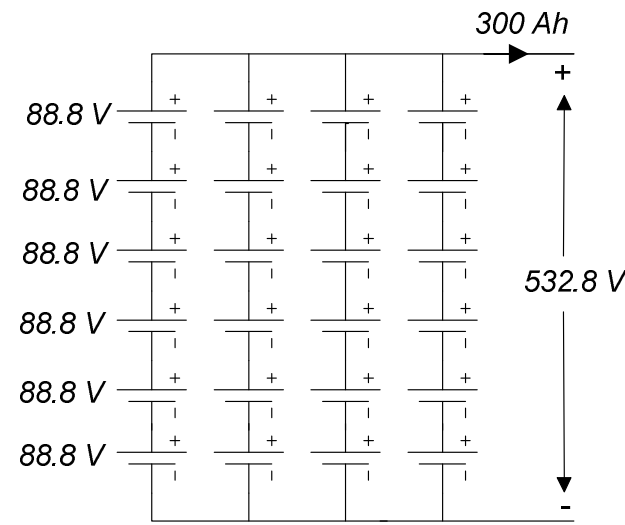

Fig. 4. Modules arrangement in each battery pack

Table 2. Battery module specifications [18]

\begin{tabular}{|c|c|c|c|}
\hline $\begin{array}{c}\text { Capacity } \\
(\mathrm{Ah})\end{array}$ & $\begin{array}{c}\text { Nominal } \\
\text { Voltage (V) }\end{array}$ & $\begin{array}{c}\text { Cycle Life at } \\
80 \% \mathrm{DoD}\end{array}$ & $\begin{array}{c}\text { Charging/Discharging } \\
\text { efficiency }\end{array}$ \\
\hline 75 & 88.8 & 15000 & 0.98 \\
\hline
\end{tabular}

Table 3. Specification of the battery pack

\begin{tabular}{|c|c|c|c|c|c|}
\hline $\begin{array}{c}\text { No. of } \\
\text { battery } \\
\text { packs }\end{array}$ & $\begin{array}{c}\text { No. of } \\
\text { Modules } \\
\text { in each } \\
\text { pack }\end{array}$ & $\begin{array}{c}\text { Current } \\
\text { Capacity } \\
\text { (Ah) of } \\
\text { each } \\
\text { pack }\end{array}$ & $\begin{array}{c}\text { Nominal } \\
\text { Voltage } \\
\text { (V) of } \\
\text { each } \\
\text { pack }\end{array}$ & $\begin{array}{c}\text { Energy } \\
\text { Capacity } \\
(\mathrm{kWh}) \text { of } \\
\text { each } \\
\text { pack }\end{array}$ & $\begin{array}{c}\text { Total } \\
\text { Energy } \\
\text { Capacity } \\
\text { of all } \\
\text { packs }\end{array}$ \\
\hline 2 & 24 & 300 & 532.8 & 159.8 & 319.6 \\
\hline
\end{tabular}

Table 4. Price of electricity [22]

\begin{tabular}{|c|c|}
\hline Category & Rate (R) \\
\hline Fixed Daily Charge & $108.191 \mathbb{C} /$ day \\
\hline Peak Period (Mon -Friday: 7am $-10 \mathrm{pm}), R_{p}$ & $27.307 \mathrm{C} / \mathrm{kWh}$ \\
\hline Shoulder Period (Sat - Sun: 7am -10pm), $R_{S}$ & $19.737 \mathrm{C} / \mathrm{kWh}$ \\
\hline Off-Peak Period (Mon - Sun: 10pm - 7am), $R_{o}$ & $11.545 \mathrm{C} / \mathrm{kWh}$ \\
\hline
\end{tabular}

Table 5. The recharging profile of the vessel

\begin{tabular}{|l|l|l|}
\hline $\begin{array}{l}\text { Charging } \\
\text { period }\end{array}$ & $\begin{array}{l}\text { No. of Recharge's per } \\
\text { day (weekday) }\end{array}$ & $\begin{array}{l}\text { No. of Recharge's per day } \\
\text { (weekend) }\end{array}$ \\
\hline Peak $n_{p}$ & 6 & 0 \\
\hline Off-peak $n_{o}$ & 1 & 1 \\
\hline Shoulder $n_{s h}$ & 0 & 6 \\
\hline
\end{tabular}




$$
\begin{aligned}
& C_{\text {weekday }}=\left[n_{p} R_{p}+n_{o} R_{o}\right] \times \mathrm{kWh} \\
& C_{\text {weekend }}=\left[n_{s} R_{s}+n_{o} R_{o}\right] \times \mathrm{kWh}
\end{aligned}
$$

Where $n_{p}, n_{o}$ and $n_{s}$ are the number of recharges per day at peak, off-peak and shoulder period respectively, $R_{p}, R_{o}$ and $R_{s}$ are the energy rate at peak, off-peak and shoulder period respectively and $\mathrm{kWh}$ is total the energy required from the grid.

Maintenance costs are estimated as a percentage of diesel system operational costs. An estimate has been taken from relevant literature due to limited information being available into the specific requirements of such large electrical systems. In this case it is deemed acceptable for electrical operational costs to be considered as $50 \%$ less than that of the current diesel system [23] which could allow for specific maintenance costs to be identified (as recharging costs were already known). Therefore, diesel maintenance costs are considered as \$AUD 23.00 per operating hour based on the ferry capacity.

\section{Battery Lifetime}

The replacing of battery packs is considered the major reoccurring cost within the electric system and thus the period before replacement was determined. Eq. 6 is used to determine this timeframe, based on a cycle life being provided by the battery manufacturer. Cycles per year are determined with each recharge required being considered as one cycle.

$$
B_{\text {lifetime }}=\frac{\text { Cycle life }}{\text { Cycles per year }}
$$

Where $B_{\text {lifetime }}$ is the lifetime of the battery in years, Cycle life is the total number of cycles at a specific DOD and Cycles per year is the number of charging cycles required by one year to charge the battery packs.

The selected battery DOD is $80 \%(20 \%$ SOC). Based on Fig. 5, the battery can be operated for 15000 cycles. When the DOD of the battery is decreased, the life cycles increases. However, the increase of the life cycles will increase the investment cost of the battery.

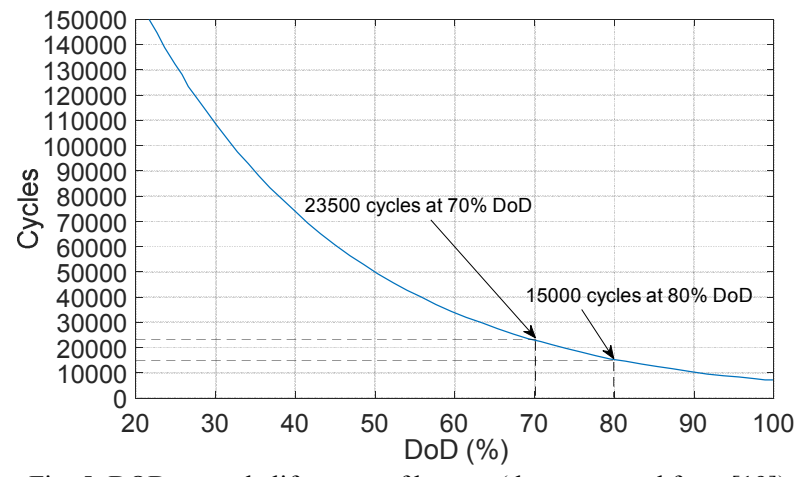

Fig. 5. DOD vs cycle life curve of battery (data extracted from [19])

\section{Payback period (PBP)}

The payback period is the length of time required to recover the ferry's initial investment costs. The economic feasibility of the concept is determined through assessment of the annual savings in operational costs against the total initial investment cost. This would yield the PBP calculated in years and provide the best indication whether the business venture is viable. It should be noted that profits from sales are assumed to be unavailable to aid in paybacks for this investment. The following equation is used to calculate the PBP:

$$
P B P=\frac{C_{\text {initial }}}{\text { Operatioanl Savings }}
$$

Where $P B P$ is the payback period in years, $C_{\text {initial }}$ is the initial investment costs (\$AUD) and Operatioanl Savings is the operational cost savings of using battery system (\$AUD) and it can be calculated by:

$$
\begin{aligned}
& \text { Operatioanl Savings } \\
& \qquad \begin{array}{l}
O C_{\text {Diesel Electric }} \\
-O C_{B \text { attery Electric }}
\end{array}
\end{aligned}
$$

Where $O C_{\text {Diesel Electric }}$ is the operational cost of using diesel electric (\$AUD) and $O C_{\text {Battery Electric }}$ is the operational cost of using battery-powered system (\$AUD).

\section{RESULTS AND DISCUSSIONS}

\section{A. Technical Results}

The proposed system is simulated in MATLAB/Simulink software to validate the calculated size of the battery against the measured load profile. The simulation is done for one round trip (3600 seconds) and the results are shown in Fig .6.
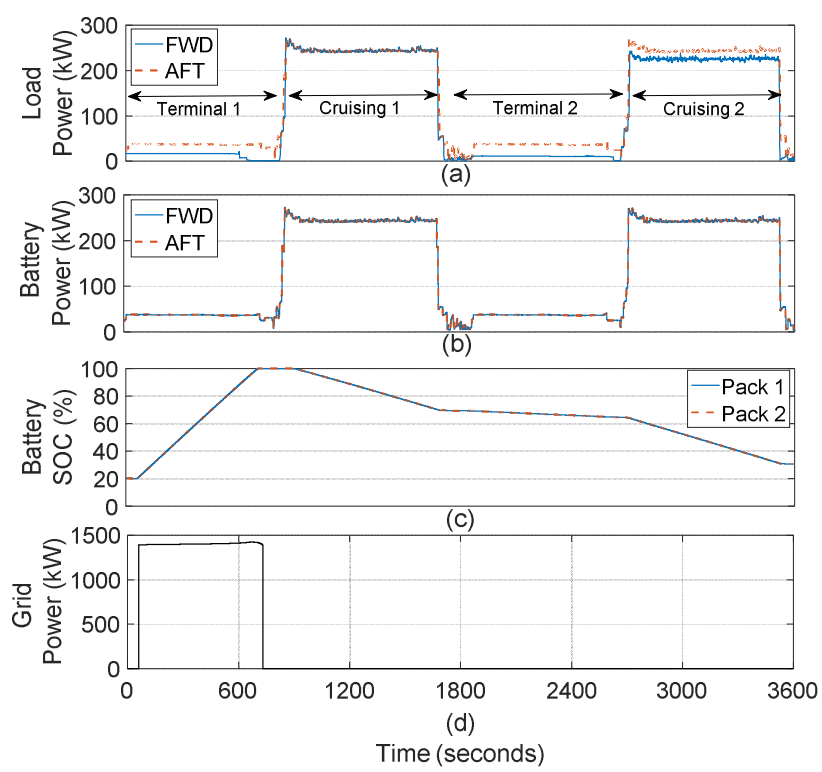

Fig. 6. Simulation results: (a) the load profiles for FES and AFT, (b) powers of FWD and AFT battery systems, (c) SOC of battery pack 1 and 2 and (d) grid power. 
Fig. 6 (a) shows the load profile of each side of the ferry and the operating conditions (Terminal 1, cruising 1, Terminal 2 and cruising 2).

When the ferry is at Terminal 1, both battery packs are in discharging mode to supply the load in the period from 0 to 60 seconds. In this period, the ferry assumed to be just berthed at the terminal and the shore connection is being connected to charge onboard battery packs as shown in Fig. 6 (b). In worth to mention that in Fig.6 (b), the powers are measured after the DC-DC converter (between the DC bus and the DC-DC converter referring to Fig. 3). In the period from 61 to 730 seconds, the shore station supply power to the ferry and charging the onboard battery packs as shown in Fig 6 (c) and (d). The grid is providing approximately $1.4 \mathrm{MW}$ in that period. Once the battery packs are fully charged, the shore supply is disconnected, and the ferry starts to departure.

At the first cruising period from 830 to 1700 seconds, the load demand of the ferry is increased as both propulsion motors are operated at higher load. Both battery packs are in discharging mode and equally shares the load demand as in Fig 6 (b) and (c). Similarly, at Terminal 2 (1700 to 2680 seconds) and cruising period 2 (2680 to 3535 seconds), both battery packs are equally sharing the load as both packs are connected to a common DC bus.

Fig. 7 shows the voltage level at the common DC bus. The DC-DC converter is responsible to maintain the DC bus voltage at $600 \mathrm{VDC}$. As it can be seen, the voltage drop is very low with a percentage of $0.3 \%$. Therefore, both DC/DC converter are successfully maintained the voltage level at the DC bus.

The simulation results revels that the selected size of the battery packs can satisfy the load demand of the battery. In addition, the SOC of each pack remained within the allowable range (more than $20 \%$ SOC or less than $80 \%$ DOD) that ensures a reasonable battery lifetime.

\section{B. Economic Results}

The total initial investment required is calculated to be $\$ 1,040,543$ with a breakdown of each element costs presented in Table 6.

The cost of the battery packs is based on the price of \$AUD 1000 per kWh. The infrastructure cost is significantly higher at \$AUD 720,863, however, this is considered a onetime investment.

Table 6. Investment costs of the battery-powered ferry

\begin{tabular}{|l|c|}
\hline Investment Elements & Cost (\$AUD) \\
\hline 2 x Battery Packs & 319,680 \\
\hline Infrastructure/Installation & 720,863 \\
\hline Total & $1,040,543$ \\
\hline
\end{tabular}

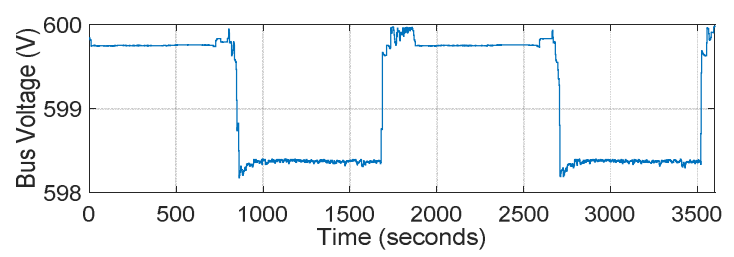

Fig. 7. DC bus voltage
The recharging costs is found to be \$AUD 133,397 by using Eq. 3. The breakdown of results for recharging costs is presented in Table 7. Weekday costs are considerably higher due to the higher amount of operating days (259 days) and having six of its seven recharging times fall within the peak period, driving costs up. As operations over weekends is limited to only Saturdays, which falls within the shoulder period, costs are relatively low.

The existing diesel electric ferry requires $60 \mathrm{~L}$ per round trip. Therefore, the total annual diesel fuel cost is \$AUD 184,111 (1 L = \$AUD 1.405). As a result, the battery powered ferry provides \$AUD 53,714 saving compared to the diesel electric system by using Eq. 8 .

A recurring annual maintenance cost of \$AUD 12,163 is found to exist for the battery electric system. This results in an annual maintenance saving of \$AUD 102,653 in comparison with the diesel electric system where the annual cost is calculated to be \$AUD 114,816. This cost reduction for the battery electric system is due to the presence of static components such as that of the battery modules and converters. The major maintenance occurring within this system will be for the cooling system, charging system (where automated parts will be featured), transformer and capacitor. The battery electric system will not require constant oil and filter changes, overhauls and associated system maintenance. Therefore, the total operational savings is SAUD 153,367. Table 8 a comparison of the operational costs between the diesel-electric and battery-powered ferry.

The payback period for the initial investment of \$AUD $1,040,543$ is calculated to be 6.7 years by using Eq. 7. The payback period in which savings increase in a linear trend each year by \$AUD 153,367 until the total savings cross the initial investment line, whereby the payback/breakeven point has been reached as shown in Fig. 8.

Table 7. Breakdown of the recharging cost

\begin{tabular}{|l|c|c|} 
Table 7. Breakdown of the recharging cost \\
\begin{tabular}{|l|c|c|}
\hline Period & Cost per day (\$AUD) & Cost per year (\$AUD) \\
\hline Weekday & 447.23 & $115,832.57$ \\
\hline Weekend & 331.41 & $17,564.73$ \\
\hline Total & 2,789 & $133,397.3$ \\
\hline
\end{tabular}
\end{tabular}

Table 8. Operational costs of diesel-electric and battery-powered ferry

\begin{tabular}{|l|c|c|c|}
\hline & $\begin{array}{c}\text { Diesel-electric } \\
\text { ferry }\end{array}$ & $\begin{array}{c}\text { Battery- } \\
\text { powered ferry }\end{array}$ & Savings \\
\hline $\begin{array}{l}\text { Diesel /electricity cost } \\
\text { (\$AUD) }\end{array}$ & 184,111 & 133,397 & $27.5 \%$ \\
\hline $\begin{array}{l}\text { Maintenance costs } \\
\text { (\$AUD) }\end{array}$ & 114,816 & 12,163 & $89.4 \%$ \\
\hline $\begin{array}{l}\text { Total operational costs } \\
\text { (\$AUD) }\end{array}$ & 298,927 & 145,560 & $51.3 \%$ \\
\hline
\end{tabular}

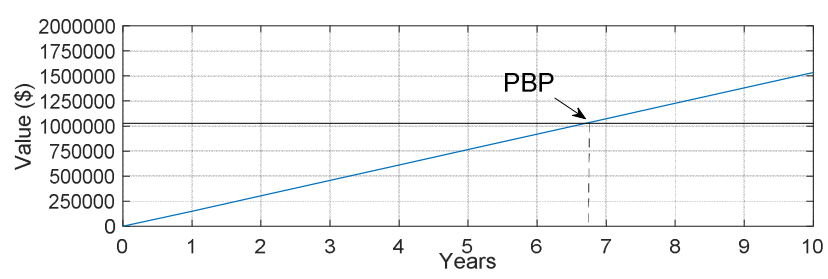

Fig. 8. Payback period plot for the electrification of MV Bowen 
Total annual savings and the PBP are expected and considered reasonable based upon previous studies surrounding the electrification of the Guemes Island ferry in the United States. Annual savings of SUSD 150,000 (1 USD $=1.35$ AUD) per year due to maintenance and energy costs were found [24] with a payback period of five years [25]. This shares similarities with the calculated values (despite slight differences in operational profiles) and indicated a level of accuracy in assumptions made within the study.

The battery lifetime with the suggested $80 \%$ DoD $(20 \%$ SOC) rate is found to be 6.8 years by using Eq. 6 . It is noted that the lifetime of the battery is almost similar to the PBP. However, with the battery size at $159.8 \mathrm{kWh}$ per pack, an actual DOD of $70 \%$ is occurring at the end of the round trip as shown in Fig. 6 (c). As per Fig. 5, when the battery is operated down to $70 \%$ DOD (30\% SOC), the lifecycle of the battery is increased to 23,500 cycles. By using Eq. 6, the associated lifetime of the battery is 10.7 years. Which is 37 $\%$ more than PBP. Battery modules will therefore require replacing four years after the initial investment is paid off with annual operational savings and hence every 10.7 years, SAUD 319,680 will be required to be invested. This reoccurring cost is assumed constant for this study, however realistically; future technological advancements should drive battery costs down while also increase longevity of battery modules.

\section{CONCLUSIONS}

This paper presents a techno-economic study for converting a diesel-electric ferry to a battery-powered ferry. The technical study includes sizing of the battery packs based on DOD and maximum load scenario. The proposed system is simulated in MATLAB/Simulink and validated against the measured load profile of the existing dieselelectric ferry. The economic study includes the investment costs, operational \& maintenance costs, and payback period.

The simulation results show that the proposed system can effectively satisfy the load demand. In addition, the battery packs are operated within the allowable DOD limits. It is noted that the maximum DOD obtained from the simulation is $70 \%(30 \% \mathrm{SOC})$. This attributes to the increase of battery life.

The economic results show that the battery-powered ferry is a feasible solution with acceptable payback period of 6.7 years. This period is considered acceptable especially as the actual DOD is found to be $70 \%$ from the simulation results. This increases the lifetime of the system to 10.7 years, which is four years after the initial investment is paid off. Battery powered solution provided a $51.3 \%$ savings of operational cost compared with diesel-electric solution. This cost reduction for the battery electric system is due to the presence of static components such as that of the battery modules and converters. The battery electric system will not require constant oil and filter changes, overhauls and associated system maintenance.

\section{ACKNOWLEDGEENT}

We would like to acknowledge Calvin Ewe for his contribution in some parts of the simulation model.

\section{REFERENCES}

[1] J. F. Hansen and F. Wendt, "Historyand Stateofthe Art in Commercial Electric Ship Propulsion, Integrated Power Systems, and Future Trends," Proceedings of the IEEE, vol. 103, pp. 2229 - 2242, 2015.

[2] M. D. Al-Falahi, T. Tarasiuk, S. G. Jayasinghe, Z. Jin, H. Enshaei, and J. M. Guerrero, "AC ship microgrids: control and power management optimization," Energies, vol. 11, pp. 1-20, 2018.

[3] M. D. Al-Falahi, K. S. Nimma, S. Jayasinghe, and H. Enshaei, "Sizing and modeling of a standalone hybrid renewable energy system," in Power Electronics Conference (SPEC), IEEE Annual Southern, 2016, pp. 1-6.

[4] E. K. Dedes, D. A. Hudson, and S. R. Turnock, "Assessing the potential of hybrid energy technology to reduce exhaust emissions from global shipping," Energy Policy, vol. 40, pp. 204-218, 2012.

[5] P. B. Christiansen, "DNV GL Annual report 2015," DNV GL13 Feb 2015.

[6] L. van Biert, M. Godjevac, K. Visser, and P. V. Aravind, "A review of fuel cell systems for maritime applications," Journal of Power Sources, vol. 327, pp. 345-364, 2016.

[7] M. D. A. Al-falahi, S. D. G. Jayasinghe, and H. Enshaei, "A review on recent size optimization methodologies for standalone solar and wind hybrid renewable energy system," Energy Conversion and Management, vol. 143, pp. 252-274, 2017.

[8] E. Skjong, R. Volden, E. Rodskar, M. Molinas, T. A. Johansen, and J. Cunningham, "Past, Present, and Future Challenges of the Marine Vessel's Electrical Power System," IEEE Transactions on Transportation Electrification, vol. 2, pp. 522-537, 2016.

[9] T. Wilberforce, Z. El-Hassan, F. N. Khatib, A. Al Makky, A. Baroutaji, J. G. Carton, et al., "Developments of electric cars and fuel cell hydrogen electric cars," International Journal of Hydrogen Energy, vol. 42, pp. 25695-25734, 2017.

[10]R. D. Geertsma, R. R. Negenborn, K. Visser, and J. J. Hopman, "Design and control of hybrid power and propulsion systems for smart ships: A review of developments," Applied Energy, vol. 194, pp. 30-54, 2017.

[11]E. A. Sciberras, B. Zahawi, and D. J. Atkinson, "Reducing shipboard emissions - Assessment of the role of electrical technologies," Transportation Research Part D: Transport and Environment, vol. 51, pp. 227-239, 2017.

[12]M. D. A. Al-Falahi, K. S. Nimma, S. D. G. Jayasinghe, H. Enshaei, and J. M. Guerrero, "Power management optimization of hybrid power systems in electric ferries," Energy Conversion and Management, vol. $172 \mathrm{C}$, pp. $50-66,2018$.

[13]R. Reabroy, Y. Tiaple, S. Pongduang, T. Nantawong, and P. Iamraksa, "The Possibility of Using Electrical Motor for Boat Propulsion System," Energy Procedia, vol. 79, pp. 1008-1014, 2015.

[14] A. M. Bassam, A. B. Phillips, S. R. Turnock, and P. A. Wilson, "Design, modelling and simulation of a hybrid fuel cell propulsion system for a domestic ferry," in PRADS2016, Copenhagen, Denmark, 2016.

[15] C. H. Choi, S. Yu, I.-S. Han, B.-K. Kho, D.-G. Kang, H. Y. Lee, et al., "Development and demonstration of PEM fuel-cell-battery hybrid system for propulsion of tourist boat," International Journal of Hydrogen Energy, vol. 41, pp. 3591-3599, 2016.

[16]J. Han, J.-F. Charpentier, and T. Tang, "An Energy Management System of a Fuel Cell/Battery Hybrid Boat," Energies, vol. 7, pp. 2799$2820,2014$.

[17] S. Trieste, S. Hmam, J. C. Olivier, S. Bourguet, and L. Loron, "Technoeconomic optimization of a supercapacitor-based energy storage unit chain: Application on the first quick charge plug-in ferry," Applied Energy, vol. 153, pp. 3-14, 2015.

[18]PBES. (2017, August 2017). System Specifications for the PBES Power \& Energy Systems. Available: http://www.pbes.com/wpcontent/uploads/2017/06/PBES Power-Energy 2017-06-16.pdf 
[19] Silvie. (2017). PBES Norway AS Announces Historic Cycle Life Milestone. Available: http://www.pbes.com/2017/01/20/pbes-norwayannounces-historic-cycle-life-milestone/

[20]K. S. Nimma, M. D. Al-Falahi, H. D. Nguyen, S. Jayasinghe, T. S Mahmoud, and M. Negnevitsky, "Grey wolf optimization-based optimum energy-management and battery-sizing method for gridconnected microgrids," Energies, vol. 11, p. 847, 2018.

[21]P. E. Will Ayers, "Comparative Payback of Lithium-Ion Batteries for Pacific NW Ferries," Elliott Bay Design Group, 2016.

[22]A. Energy. (2018). Tariff-94 Business Time of Use (ToU) Price FactSheet. Available: https://www.auroraenergy.com.au/Aurora/media/pdf/small to mediu $\underline{m \text { business/Tariff-94-Business-Time-of-Use-(ToU)- }}$ Price FactSheet.pdf

[23] K. E. Holmefjord. (2017) All-Electric: The Future of Ferry Propulsion. Pacific Maritime Magazine. Available: http://www.pacmar.com/story/2017/03/01/features/all-electric-thefuture-of-ferry-propulsion/497.html

[24]B. Stone. (2015). County considers electric ferry. Available: https://www.goskagit.com/skagit/county-considers-electricferry/article 64cb5f15-f1d1-5bbe-8d4e-a9285cfcab71.html

[25] A. ASSOCIATES. (2016, 29 January). Journey to All-Electric Ferry Development. Available: https://www.masstransitmag.com/press_release/12163841/journey-toall-electric-ferry-development 


\section{Chapter 4: \\ Application of Classical and Meta-Heuristic-Based Optimization for Electric Ferries}

This chapter presents design and application of the meta-heuristic optimization algorithm namely GWO for hybrid electric short-haul ferries. The performance of GWO is compared with that of classical rule-based method and assessed in terms of fuel consumption and emission reduction. Moreover, the fuel consumption of the proposed hybrid system with both PMS methods is compared with that of the existing AC power system of the ferry. This chapter has been published in the Journal of Energy Conversion and Management. The citation of the article is

Al-Falahi, M. D., Nimma, K. S., Jayasinghe, S. D., Enshaei, H., \& Guerrero, J. M. (2018). Power management optimization of hybrid power systems in electric ferries. Energy Conversion and Management, 172, 50-66. 


\title{
Power management optimization of hybrid power systems in electric ferries
}

\author{
Monaaf D.A. Al-Falahi ${ }^{\mathrm{a}, *}$, Kutaiba S. Nimma ${ }^{\mathrm{a}}$, Shantha D.G. Jayasinghe ${ }^{\mathrm{a}}$, Hossein Enshaei ${ }^{\mathrm{a}}$, \\ Josep M. Guerrero ${ }^{\mathrm{b}}$ \\ a Australian Maritime College, University of Tasmania, Newnham, TAS 7248, Australia \\ ${ }^{\mathrm{b}}$ Institute of Energy Technology, Aalborg University, Aalborg 9220, Denmark
}

\section{A R T I C L E I N F O}

\section{Keywords:}

Battery

DC power system

Electric ferry

Energy storage system

Hybrid power system

Power management

\begin{abstract}
A B S T R A C T
The integration of more-electric technologies, such as energy storage systems (ESSs) and electric propulsion, has gained attention in recent years as a promising approach to reduce fuel consumption and emissions in the maritime industry. In this context, hybrid power systems (HPSs) with direct current (DC) distribution are currently gaining a commendable interest in research and industrial applications. This paper examines the impact of using HPS with DC distribution and a battery energy storage system (BESS) over a conventional AC power system for short haul roll-on/roll-off (RORO) ferries. An electric ferry with a HPS is modeled in this study and the power management system is simulated using the Matlab/Simulink software. The result is validated using measured load profile of a ferry. The performance of the DC HPS is compared with the conventional AC system based on fuel consumption and emission reductions. An approach to estimate the fuel consumption of the diesel engine through calculation of specific fuel oil consumption (SFOC) is also presented. This study uses two optimization techniques: a classical power management method namely Rule-Based control (RB) and a meta-heuristic power management method known as Grey Wolf Optimization (GWO) to optimally manage the power sharing of the proposed HPS. Fuel consumption and emission indicators are also used to assess the performance of the two power management methods. The simulation results show that the HPS provides a $2.91 \%$ and $7.48 \%$ fuel consumption reduction using RB method and GWO method respectively. It is apparent from the result that the HPS has more fuel savings while running the diesel generator sets (DGs) at higher operational efficiency. It is interesting that the proposed HPS using both power management methods provided a 100\% emission reduction at berth. Finally, it was found that using a meta-heuristic optimization algorithm provides better fuel and emission reductions than a classical method.
\end{abstract}

\section{Introduction}

Emission regulations imposed by the international marine organization (IMO), along with growing concerns on the environment, are causing a major shift in the industry's approach to propulsion system design and increasing the demand for environmentally friendly marine power system solutions $[1,2]$. In addition, the fluctuation of oil prices required the incentive to investigate more technologically advanced and efficient solutions to reduce operational expenses in the transportation industry $[3,4]$. Therefore, the industry has collectively been exploring other opportunities for emissions control and energy savings which range from burning low emission fuels such as liquefied natural gas [1] and using dual fuel [5] to progressively electrify ships through increasing hybridization [6]. In the same context, the IMO suggested the concept of hybrid electric vessels as one of the energy efficient index to control and limit a vessel's emissions [7]. This has opened up the integration of energy storage systems (ESSs) and renewable energy sources (RESs) into ship power systems [8,9].

As the overwhelming majority of present electric vessels use AC distribution systems, the hybridization of ship power systems is complex as synchronization of each generation unit is required. In addition, ship AC distribution systems have drawbacks such as inrush current of transformers, three-phase imbalances, harmonic currents, and reactive power flow [10]. On the other hand, a DC distribution system provides an efficient distribution of electric energy by linking AC and DC energy sources through power-electronic devices which customize energy flow to the load $[11,12]$. However, power electronic converters add complexity to the system due to their non-linear characteristics and switching behavior $[13,14]$. Nevertheless, the recent progressive developments of power electronics devices make them more reliable and

\footnotetext{
* Corresponding author.

E-mail addresses: monaaf.alfalahi@utas.edu.au (M.D.A. Al-Falahi), kutaiba.sabah@utas.edu.au (K.S. Nimma), shanthaj@utas.edu.au (S.D.G. Jayasinghe), hossein.enshaei@utas.edu.au (H. Enshaei), joz@et.aau.dk (J.M. Guerrero).
} 


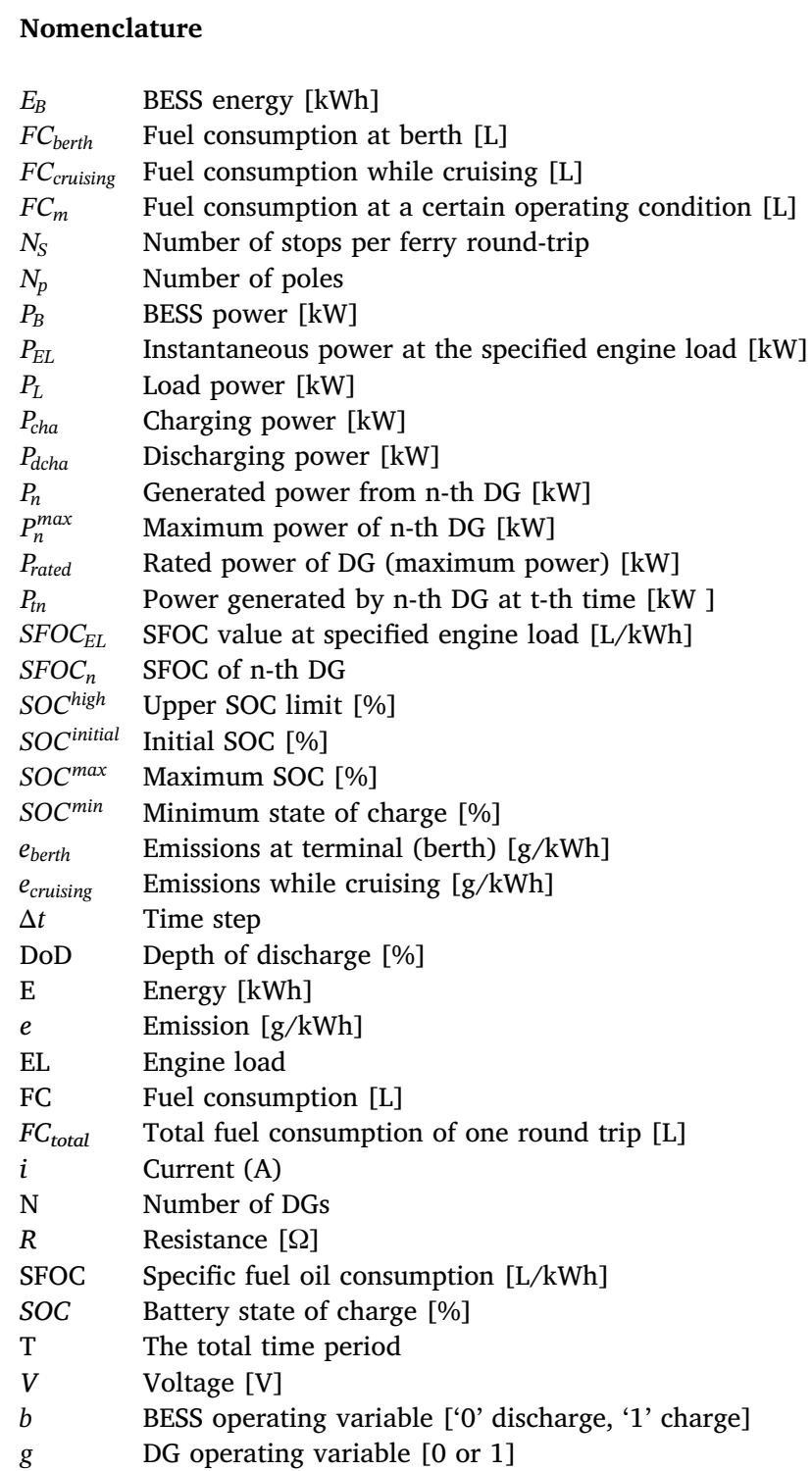

$\begin{array}{ll}\eta & \text { Efficiency } \\ \theta e & \text { Electrical angle } \\ \theta r & \text { Rotor angle } \\ \lambda & \text { Ratio of load }\end{array}$

Abbreviation

$\begin{array}{ll}\text { BESS } & \text { Battery energy storage system } \\ \mathrm{CO}_{2} & \text { Carbon dioxide } \\ \mathrm{DG} & \text { Diesel generator-set } \\ \text { ESS } & \text { Energy storage system } \\ \text { GWO } & \text { Grey wolf optimization } \\ \mathrm{HPS} & \text { Hybrid power system } \\ \mathrm{IMO} & \text { International marine organization } \\ \mathrm{NO}_{\mathrm{X}} & \text { Nitrogen oxide } \\ \mathrm{PMS} & \text { Power management strategy } \\ \mathrm{RB} & \text { Rule-based } \\ \mathrm{RES} & \text { Renewable energy source } \\ \mathrm{RORO} & \text { Roll-on/roll-off } \\ \mathrm{SO}_{\mathrm{X}} & \text { Sulfur oxides }\end{array}$

Subscripts

$\begin{array}{ll}t_{c} & \text { The index time of charging } \\ B & \text { Battery } \\ \text { bus } & \text { Bus } \\ \text { cha } & \text { Charging } \\ \text { discha } & \text { Discharging } \\ \text { EL } & \text { Engine load } \\ L & \text { Load } \\ \text { loss } & \text { Power loss } \\ m & \text { Operating condition } \\ \text { max } & \text { Maximum } \\ \text { min } & \text { Minimum } \\ n & \text { n-th DG } \\ \text { prop } & \text { Propulsion } \\ \text { ref } & \text { Reference } \\ r m s & \text { Root-mean square } \\ \text { serv } & \text { Service } \\ \mathrm{t} & \text { t-th time interval } \\ T & \text { Terminal }\end{array}$

efficient. This makes the DC distribution is more feasible in various applications [12]. Therefore, the use of a HPS with DC distribution enables easier integration of RESs and ESSs $[10,15]$. In addition, synchronization of generation units is not required which enables the prime movers to operate at their optimal speeds providing a reduction of fuel consumption and emissions [10,16]. This also offers further advantages, such as space and weight savings, flexible arrangement of equipment and noise reduction from a diesel gen-set (DG) in the harbor $[17,18]$. Moreover, retrofitting of a conventional marine power system with emerging renewable energy and energy storage technologies provides significant cost and environmental benefits [9,19,20]. As a result, the transition from a ship power system with AC distribution to a HPS with DC distribution is gaining more attention $[12,17]$.

The aforementioned advantages of a HPS with DC distribution give an efficient power system solution for short-haul ferries as most ferries operate closer to urban areas where the reduction of noise and emissions is required [21]. As most of the ferries use fossil fuels such as diesel to produce on-board power, they produce pollutant emissions, include $\mathrm{CO}_{2}, \mathrm{NO}_{\mathrm{X}}, \mathrm{SO}_{\mathrm{X}}$ and particular matter [22,23]. When a ferry is berthed at a terminal, these emissions occur close to human habitation and result in a more direct impact on health [24]. Moreover, ferries account for a significantly high percentage of in-port emissions based on frequencies of calls compared to other types of vessels [25]. Such greenhouse gas emissions have a significant risk on human health including chronic bronchitis, heart disease, stroke and respiratory tract infection [22]. Therefore, policy makers have explored and introduced several methodologies in limiting port emissions based on port structural changes $[26,27]$. A cold-ironing method can be considered as a common solution to reduce in-port emissions and noise at terminals [28]. This method uses shore power to supply power to the on-board engines [29]. However, sometimes the shore power supply uses nonrenewable energy sources [30]. In addition, economic factors need to be taken into account to justify investment in a shore power station as short-haul ferries usually berth for short period [28]. Therefore, there should be a more reliable solution to eliminate in-port emissions from ferries. Thus, all-electric and hybrid-electric ferries are practically achievable and the integration of RESs greatly reduces their emissions and fuel consumption. However, the slow dynamics or intermittent nature of RESs prevents them being the main source of power in ferries. Thus, a battery energy storage system (BESS) has become an integral part in such systems to ensure a reliable supply of power [31]. Therefore, the trend towards integration of the BESS into ferries has gained more attention in recent years. For example, MV Hallaig, the first hybrid electric ferry with battery storage, started operation in 2013 
recording significant fuel savings and emission reductions [32]. Following the same trend in fuel and emissions reduction, Ampere ferry, the first battery powered ferry in the world, started operation in 2015 and reported a significant fuel savings with zero emission operation $[17,21,33]$. This trend is continuing as more ferries are being built with hybrid and fully battery powered systems owing to their advantage of emission reductions, especially as most ferries operate close to human habitation areas $[11,21,32,33]$.

The DC HPS with a BESS can be considered a promising solution to reduce emissions and noise in harbors to significantly low levels. In order to increase the potential of such a system, an efficient power management strategy is essential which can optimally share power among all HPS components. In this context, modeling a simulation platform is vital to derive an efficient power sharing strategy and thereby achieve fuel savings and emission reductions. Power and size optimization approaches for land-based HPSs have been extensively discussed $[34,35]$. However, modeling, simulation and power management optimization of electric ferries with HPSs have not been extensively discussed. Only a few studies have discussed the use of HPSs in domestic ferry and boat applications, which mostly used classical and deterministic PMS methods [36-41]. In [36], the authors have studied and designed a hybrid fuel cell electric propulsion system for a domestic ferry and compared it with the performance of the existing diesel propulsion system. In [37], the development and demonstration of a fuel cell/battery hybrid system for a tourist boat is presented. In [38], the authors have investigated the effectiveness of using a hybrid system with battery in a passenger ferry. In [39], the authors proposed a hybrid fuel cell/battery power system for a low power boat. A classical energy management system, namely a state-based method, is used to manage the power generation. In [40], authors proposed an energy management system based on a deterministic state-based control method to manage the energy of a hybrid fuel cell/battery passenger ferry. In [41], the authors presented a techno-economic approach to minimize the overall cost of an ESS in a supercapacitor plug-in ferry. The aforementioned studies have not considered modeling, simulation, and evaluation of a hybrid domestic ferry with DC distribution and a BESS in terms of fuel savings and emission reductions. Moreover, to the best of our knowledge, the power management of DC HPSs for short haul ferries integrated with a BESS using a meta-heuristic method has not been reported in the existing literature.

The contributions of this paper can be summarized as follows:

- Performance comparison of using a hybrid DC over a conventional AC power system for short-haul ferries in terms of fuel consumption and emissions reductions;

- An approach to estimate the fuel consumption through the SFOC of a diesel engine;

- Optimal management and exploitation of generation and BESS for fuel consumption, greenhouse gas emissions and in-port noise reductions;

- Design, application and comparison of classical (RB method) and meta-heuristic (GWO) power management methods to optimally manage the power generation in hybrid ferries.

In order to examine and validate the proposed HPS system, a measured load profile of an existing ferry in Tasmania, Australia, is used.

The paper is organised as follows. The performance indicators used to evaluate the proposed system and PMS are presented in Section 2. The PMSs used in this study to optimally manage the power of the hybrid ferry are presented in Section 3. Modelling of the the proposed system components is given in Section 4. The case study used and the corresponding proposed HPS are presented in Section 5. Results of the simulation and analysis are presented in Section 6 to demonstrate the effectiveness of HPS over AC system for short-haul ferry application. Finally, conclusions drawn from the results of this study are given in
Section 7.

\section{Performance indicators}

\subsection{Fuel consumption}

The presence of dynamic loads in marine power systems makes marine diesel engines operate at changing conditions. As a result, engines are not operated at their optimum loading conditions which in turn increases the fuel consumption [42]. SFOC is a measure of the fuel efficiency and fuel savings of any prime mover that burns fuel and produces power [43]. An SFOC curve can be used to identify the optimum operating region of a given engine and thereby take measures to improve the fuel consumption. Typically, the optimum loading range for diesel engines is within $60 \%$ to $100 \%$ of the rated engine power [44]. Operating the engine in this range will significantly reduce the SFOC to lower levels.

The SFOC can be used to estimate the fuel consumption of the onboard engines. Several methods are available in the literature to estimate the fuel consumption of marine engines $[43,45,46]$. Generally, these methods are used to estimate the fuel consumption and emissions of main and axillary engines of large marine vessels with long voyages and several route options. In this context, recognized values of SFOC and emissions factors are essential to estimate the fuel consumption and emissions [47]. Under those circumstances, the traffic emissions assessment model (STEAM2) is used to estimate emissions and fuel consumption of a ship's main and axillary engine [43]. In a STEAM2 model, several data inputs are required such as ship speed, load profile, ship movement engine loads and fuel changes. Three relative SFOC for medium and large size engines provided by manufacturers were used. It was found that the relative SFOC curve of all three engines has the parabolic shape as shown in Fig. 1. This results in the conclusion that minimizing fuel oil consumption and improving the performance of engines can be achieved by running the engines at high engine loads.

In this paper, a simple approach to estimate the fuel consumption for short-haul ferries is proposed. The proposed approach is similar to [43] in some aspects. However, in this approach, the SFOC in $\mathrm{L} / \mathrm{kWh}$ is estimated rather than using a relative SFOC. In addition, in this approach, only the load profile is required to estimate the SFOC which is then used to calculate the fuel consumption of the engine. The applicability of the proposed approach is validated through a real case study described in Section 5.

The fuel consumption in $\mathrm{L} / \mathrm{h}$ at different engine loads is extracted from the manufacturer's data sheet [48]. The SFOC (L/kWh) curve shown in Fig. 2 is derived by dividing the fuel consumption at each engine load by the rated engine power $(\mathrm{kW})$.

The parabolic shape of the SFOC curve shown in Fig. 2 can be represented by a second degree polynomial function (quadratic function):

$y=a x^{2}+b x+c$

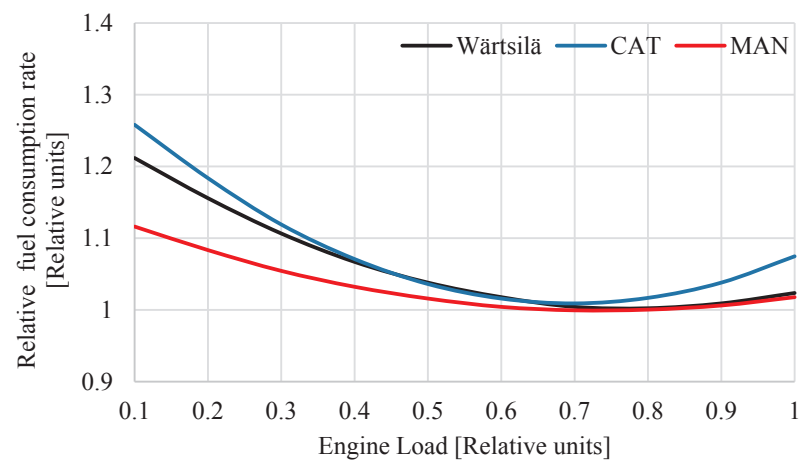

Fig. 1. The relative SFOC based on data of three manufacturers: Wärtsilä, Caterpillar and MAN [43]. 


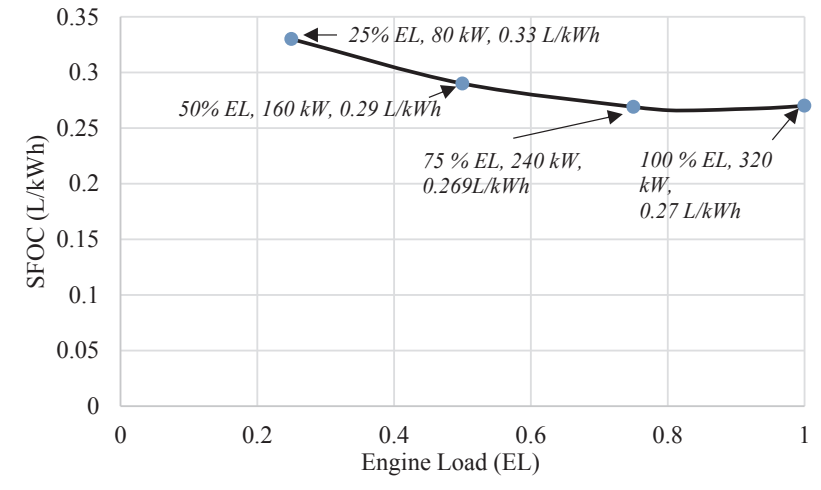

Fig. 2. The SFOC curve of the $320 \mathrm{~kW}$ Cummins Gen-Set (Model C350 D6).

where $a, b$ and $c$ are the coefficients of the equation, $x$ is the engine load and $y$ is the SFOC.

By using regression estimation, the coefficients of the second degree polynomial equation for the SFOC are calculated and presented in Table 1.

Therefore, the derived quadratic equation for the SFOC can be expressed as:

$S F O C=0.1691 E L^{2}-0.2924 E L+0.3929$

where $E L$ is the engine load expressed by:

$E L=\frac{\text { GeneratedPower }(k W)}{\text { EngineRatedPower }(k W)} ; 0 \leq E L \leq 1$

The total fuel consumption in liters $\left(\mathrm{FC}_{\text {total }}\right)$ of the ferry for a complete round trip is the summation of fuel consumption at each operating condition:

$F C_{\text {total }}=F C_{\text {berth }}+F C_{\text {cruising }}$

where $F C_{\text {berth }}$ is the fuel consumption when the ferry is berthed (at terminal) and $F C_{\text {crusing }}$ is the fuel consumption when the ferry is cruising.

The fuel consumption in liters at any operational mode can be calculated by:

$F C_{m}=S_{F O C_{E L}}\left(P_{E L} \times t_{m}\right)$

where $m$ represents the ferry operation mode (berth or cruising), $S F O C_{E L}$ is the value of SFOC at a specified engine load, $P_{E L}$ in the instantaneous generated power at the specified engine load, and $t_{m}$ is the time duration in hours at the specified engine load.

\subsection{Emission reductions}

Emission reduction is an important factor in maritime transportation. Many inventories have been introduced to calculate and estimate emissions from marine vessels $[47,49]$. Generally, estimation is based on activity and/or fuel consumption. An activity-based approach requires detailed data such as ship speed, engine workload, routing, location, time information, ship profile and duration [50,51]. Therefore, the activity-based approach is generally used to estimate the emissions from large ships [51]. A fuel-based approach is a top-down method to estimate emissions based on fuel consumption. In this approach, the fuel consumption/energy consumption is required to estimate emissions [51]. This paper uses the Entac inventory with the top-down method, as it covers the emissions estimations from ferries and requires only the load profile of the engine to calculate the emissions [52]. In this method, the $\mathrm{CO}_{2}, \mathrm{SO}_{\mathrm{X}}$ and $\mathrm{NO}_{\mathrm{X}}$ emissions are estimated as a function of the vessel energy consumption multiplied with an emission factor at each operating condition [51,53]. Table 2 provides the equations used in estimating the emissions [52].

\section{Power management strategy (PMS)}

A power management unit is essential in order to optimally reduce the operating hours of the diesel engines, run the engines at their maximum efficiency and maintain the battery state of charge (SOC) at a certain level. In this paper, deterministic control method, namely rulebased (RB) control strategy, and a meta-heuristic on-line optimization method, namely Grey Wolf Optimization (GWO), are proposed and implemented. Fuel consumption and emission reductions are used as indictors to investigate the performance of each method.

\subsection{Rule-based (RB) strategy}

This strategy uses pre-determined operational conditions (states) to control the power sharing among the two diesel-generator sets (DGs) and the BESS. The advantages of this deterministic RB method are a low computational burden on the processor and relatively simple implementation [54]. Nevertheless, there can be performance degradations as it uses pre-determined states which could vary over time $[40,55]$.

Several operating states based on battery SOC and total load power are defined in order to control the power sharing of each component. The flow chart of the proposed RB PMS is shown in Fig. 3. The input variables are battery SOC and total load power $\left(P_{L}\right)$. Outputs are the decisions to switch the DGs on/off and charge/discharge the BESS. When the SOC is within the lower boundary $\left(\mathrm{SOC}^{\mathrm{min}} \leq S O C<S O C^{\text {high }}\right)$ and the load power exceeds a certain limit $\left(P_{L}^{\min }\right)$, the DG starts to supply power and shuts down as soon as the SOC exceeds the upper limit $\left(S O C^{\text {high }}\right)$. During low power demand $\left(P_{L} \leq P_{L}^{\min }\right)$ when the ferry is at berth, the BESS operates in discharge mode to supply the load demand and both DGs are shut down. During medium power demand $\left(P_{L}^{\min }<P_{L} \leq P_{L}^{\text {ave }}\right)$, only one DG operates and the BESS is either charging or discharging depending on the SOC value. At high load demand $\left(P_{L}>P_{L}^{a v e}\right)$, both DGs operate and the BESS is either at standby mode or at charging mode depending on the value of SOC.

\subsection{Grey wolf optimization (GWO)}

The use of meta-heuristic optimization techniques has gained huge attention over the last two decades. This is due to their capability of solving multi-objective optimization problems with several constraints. This provides better quality results compared to classical optimization techniques [34]. In this context, a meta-heuristic optimization technique, namely Grey Wolf Optimization (GWO), is implemented. The GWO is a population-based meta-heuristic swarm intelligence technique. This optimization technique was proposed in 2014 by Mirjalili [56]. Several studies have implemented GWO and compared its results with other algorithms. These studies found that GWO provides competitive optimization results compared to other swarm and evolutionary algorithms such as particle swarm optimization (PSO) [56-58], differential evolution (DE) [56], gravitational search algorithm (GSA) [56,57], genetic algorithm (GA) [58] and ant colony optimization (ACO) [59].

This algorithm mimics the social behavior of the grey wolf. Grey wolves live and hunt in groups of 5 to 12 individuals. The social hierarchy of the grey wolves is represented in Fig. 4. The highest level of the hierarchy contains the leader of the wolf pack, represented as alpha $(\alpha)$. The leader is responsible for making decisions to hunt, wake and sleep. The second level in the hierarchy is called beta $(\beta)$. These wolves are

Table 1

The coefficients of the SFOC equation.

\begin{tabular}{llll}
\hline Coefficient & $\mathrm{a}$ & $\mathrm{b}$ & $\mathrm{c}$ \\
\hline Value & 0.1691 & -0.2924 & 0.3929 \\
\hline
\end{tabular}


Table 2

Emissions estimation equations (summarized from [52]).

\begin{tabular}{|c|c|c|c|}
\hline & $\begin{array}{l}\mathrm{CO}_{2} \text { emissions, } \mathrm{e}_{\mathrm{CO} 2} \\
(\mathrm{~g} / \mathrm{kWh})\end{array}$ & $\begin{array}{l}\mathrm{SO}_{\mathrm{X}} \text { emissions, } \mathrm{e}_{\mathrm{SOX}} \\
(\mathrm{g} / \mathrm{kWh})\end{array}$ & $\begin{array}{l}\mathrm{NO}_{\mathrm{X}} \text { emissions, } \mathrm{e}_{\mathrm{NOX}} \\
(\mathrm{g} / \mathrm{kWh})\end{array}$ \\
\hline Berth & $682 \times$ output energy & $11.6 \times$ output energy & $12 \mathrm{x}$ output energy \\
\hline Cruising & $620 \times$ output energy & $10.5 \mathrm{x}$ output energy & $15 \mathrm{x}$ output energy \\
\hline
\end{tabular}

considered as consultants to the $\alpha$ wolf which are considered as the second best wolves in the pack. They convey the orders to the lower level wolves and send feedback of low level wolves to $\alpha$ wolf. The third level wolves are called delta $(\delta)$. They obey instructions from the $\alpha$ and $\beta$ wolves. The lowest level in the hierarchy is omega $(\omega)$ wolves and their role is only to follow the orders of the higher-level wolves.

One of the important social activities of grey wolves is hunting. The steps of this process include: (i) tracking, chasing and approaching the prey, (ii) pursuing, encircling and harassing the prey; and (iii) attacking the prey [56]. In order to mathematically represent the social hierarchy and hunting technique of grey wolves $\alpha$ is considered as the best solution, $\beta$ the second best solution (mean solution), $\delta$ is the third best solution (worst solution) and $\omega$ is the other solutions. The first step in hunting is encircling the prey. The equations of this behavior are [56]:

$D^{\rightarrow}=\left|C^{\rightarrow} \cdot X_{p}^{\rightarrow}(\mathrm{t})-\mathrm{X}^{\rightarrow}(t)\right|$

$X^{\rightarrow}(t+1)=X_{p}^{\rightarrow}(t)-A^{\rightarrow} . D^{\rightarrow}$

where $D^{\rightarrow}$ is a calculated vector used to specify a new position of the wolf, $\mathrm{X} \rightarrow$ is the position vector of the wolf, and $X_{p}^{\rightarrow}$ is the position of the

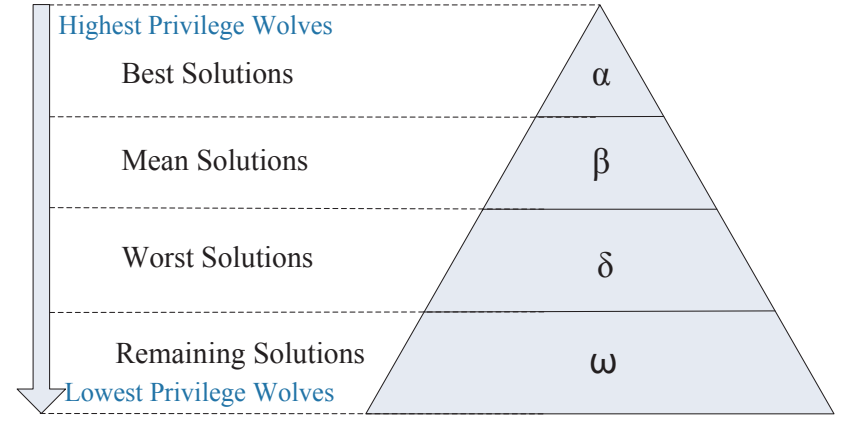

Fig. 4. The social hierarchy of grey wolves.

prey $A^{\rightarrow} . D^{\rightarrow}$ are coefficient vectors calculated by [56]:

$A^{\rightarrow}=2 \vec{a} \cdot r_{1}-a^{\rightarrow}$

$C^{\rightarrow}=2 \cdot r_{2}^{\rightarrow}$

where $a^{\rightarrow}$ is a vector set to decrease linearly from 2 to 0 over the iterations and $r_{1}$ and $r_{2}$ are random vectors in [0,1].

As mentioned earlier, only the alpha wolf guides the hunting process. Therefore, it is considered the best solution. Beta and delta wolves are participating and assisting in the hunting process. Therefore, alpha, beta and delta are considered as the three first solutions. Then the other search agents update their positions according to the best search agents. The new position vector of each wolf is calculated by the following equations [56]:

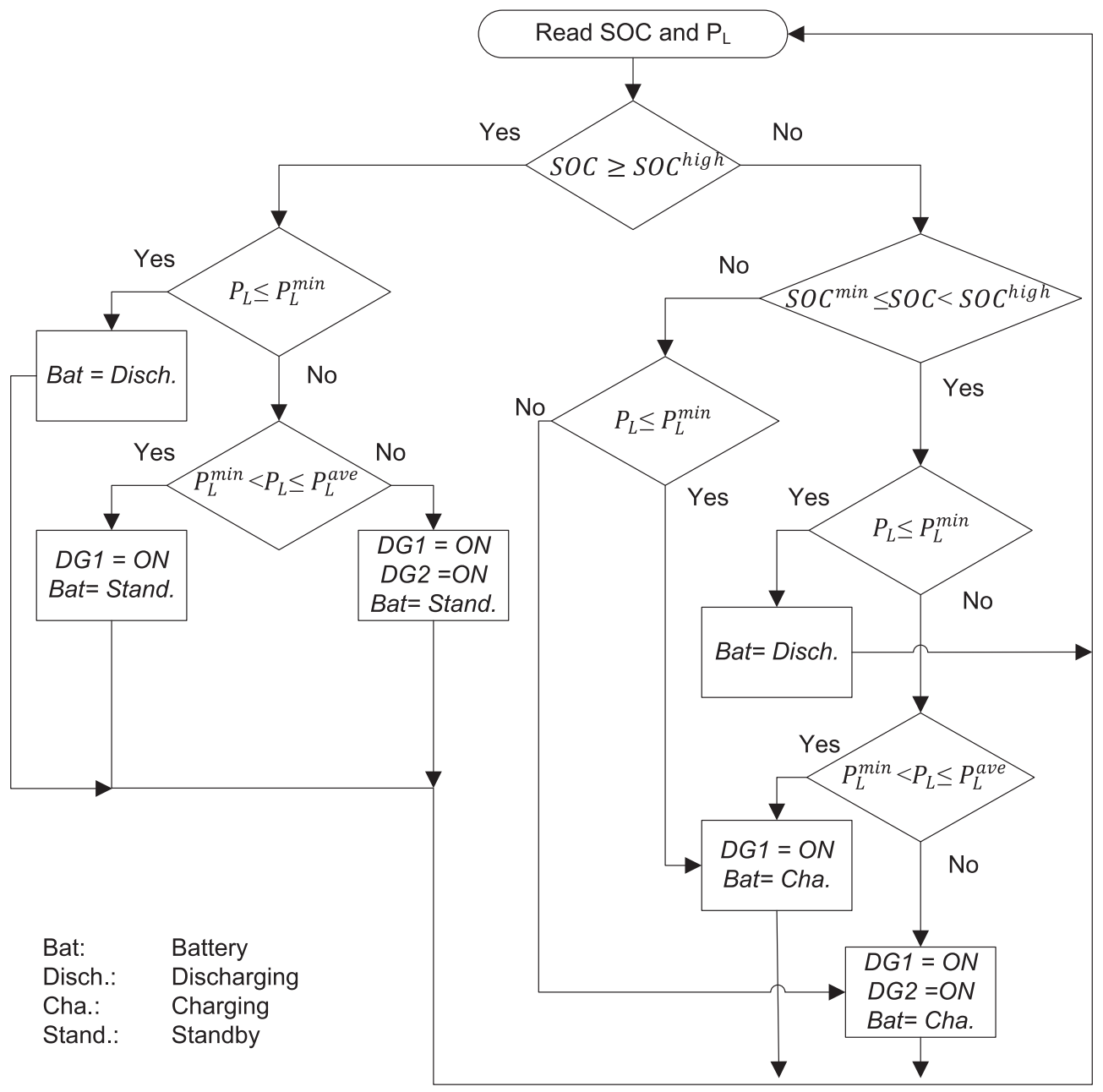

Fig. 3. Flowchart of the proposed rule-based (RB) PMS. 
$D_{A l p h a}=\mid C_{1} \cdot X_{A l p h a}-X \rightarrow$

$D_{\text {Beta }}^{\vec{a}}=\left|C_{2} \overrightarrow{X_{\text {Beta }}}-X^{\rightarrow}\right|$

$\overrightarrow{D_{\text {Delta }}}=\left|C_{3} \cdot X_{\text {Delta }}-X^{\rightarrow}\right|$

$X_{1} \overrightarrow{=}\left|X_{A l p h a}-A_{1} \cdot D_{A l p h a}\right|$

$X_{2}^{\rightarrow}=\left|X_{\text {Beta }}-A_{2} \cdot \overrightarrow{D_{\text {Beta }}}\right|$

$X_{3}=\left|X_{\text {Delta }}^{\vec{A}}-A_{3} \cdot \overrightarrow{D_{\text {Delta }}}\right|$

$X^{\rightarrow}(t+1)=\frac{X_{1}^{\rightarrow}+X_{2}^{\overrightarrow{ }}+X_{3}^{\rightarrow}}{3}$

where $\overrightarrow{\mathrm{D}_{\text {Alpha }}}, \overrightarrow{\mathrm{D}_{\text {Beta }}}$ and $\overrightarrow{\mathrm{D}_{\text {Delta }}}$ are calculated vectors used to specify new positions of the wolf, $\mathrm{X}_{\text {Alpha }}, \mathrm{X}_{\text {Beta }}$ and $\mathrm{X}_{\text {Delta }}$ are the vectors of the grey

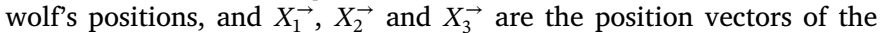
wolves.

Alpha, beta and delta wolves estimate the possible positions of the prey while the simulation is running. The alpha solution is used as a final solution as it always provides the optimal (best) solution-set compared to beta and delta.

\subsubsection{GWO application on a hybrid electric ferry}

The GWO tool is used to solve the power management optimization of the short-haul hybrid ferry. The main objective function of the optimization is to minimize the fuel consumption of DG1 and DG2. The optimization parameters are the DG1 power, DG2 power and battery power. Optimizing these parameters will optimize the value of SFOC and results in reduction of fuel consumption and emissions. The DG1 and DG2 powers are optimized based on running at least one DG at the optimal operating point and ensuring that the other DG is operated above the low operational efficiency region. This can be achieved by uniformly charging the battery in order to keep the engine operating at highest engine load over the entire cruising period. In addition, fuel consumption minimization includes shutting down the DGs at low load demand (at terminal) as DGs are required to operate only at higher load demand. This operation will eliminate the noises (in addition to emissions elimination) at terminal as both DGs are not operating. Therefore, emissions and noise reductions at berth (terminal) are then incorporated in the fuel consumption minimization. The main objective function of the fuel consumption minimization is presented as follow:

$F C_{\text {total }}=\sum_{t=1}^{\mathrm{T}} \sum_{n=1}^{\mathrm{N}}\left(\operatorname{SFOC}_{n} \cdot P_{t n} \cdot g_{t n} \cdot \Delta t\right)$

where $F C_{\text {total }}$ is the total fuel consumption of the ferry (L), $P_{t n}$ is the power generated by n-th DG at t-th time (kW), $g_{t n}$ is the DG operating variable ( 0 is "OFF" or 1 is "ON"), $\Delta t$ is the time step, $t$ is t-th time interval, $N$ is the number of DGs and $S F O C_{n}$ is the specific fuel oil consumption of $\mathrm{n}$-th DG represented by the following equation,

$\mathrm{SFOC}_{n}=\left[a\left(\frac{P_{t n}}{P_{\text {rated }}}\right)^{2}-b\left(\frac{P_{t n}}{P_{\text {rated }}}\right)+c\right] \cdot\left(P_{t n} \times t\right)$

where a,b and c are the coefficients of the SFOC equation and $P_{\text {rated }}$ is the DG rated power $(\mathrm{kW})$.

The optimization objective function is subjected to the following constraints:

\section{- Power balance constraint}

The power supplied from the generation side must be equal to the load demand for any period $t$,

$\sum_{t=1}^{\mathrm{T}} \sum_{n=1}^{\mathrm{N}}\left(P_{t n}+P_{B, t}\right)=P_{L, t}$

where $P_{B, t}$ is the BESS power at t-th time (kW) and $P_{L, t}$ is the load power at $\mathrm{t}$-th time.

\section{- Power constraints of DG units}

The power generated from each DG must be within the allowable limit

$P_{n}^{\min } \leq P_{n} \leq P_{n}^{\max }$

where $P_{n}$ is the power generated by n-th generator (kW), and $P_{n}^{\min }$ and $P_{n}^{\max }$ are the minimum and maximum power limit of the DGs (kW).

\section{- BESS constraints}

The battery power must be within the allowable limit. The maximum and minimum power of the BESS is determined based on the battery datasheet and complying with the load profile. These limits can be obtained by proper sizing of the BESS based on the measured load profile. The BESS constraints are as follow:

$P_{B}^{\min } \leq P_{B} \leq P_{B}^{\max }$

$P_{d c h a}^{\min } \leq P_{d c h a} \leq P_{d c h a}^{\max }$

$P_{\text {cha }}^{\min } \leq P_{\text {cha }} \leq P_{\text {cha }}^{\max }$

$E_{B}^{\min } \leq E_{B} \leq E_{B}^{\max }$

$S O C^{\min } \leq S O C \leq S O C^{\max }$

where:

$P_{B, t}=P_{d c h a, t} \times\left(1-b_{t}\right)-P_{c h a, t} \times b_{t}$

$P_{d c h a, t}=\frac{P_{L, t}}{\eta_{d c h a}} ; P_{L, t} \leq P_{c h a}^{\max }$

$P_{\text {cha }, t}=\left(1-\frac{E_{B, t_{c}}}{E_{B}^{\max }}\right) \times P_{\text {cha }}^{\max } \times \eta_{\text {cha }} ; P_{L, t}>P_{\text {cha }}^{\max }$

$E_{B, t}=E_{B, t-1}+\left[P_{\text {dcha }, t} \times\left(1-b_{t}\right)-P_{\text {cha }, t} \times b_{t}\right] . \Delta t$

$S O C_{t}=\left(\frac{E_{B, t}}{E_{B}^{\max }}\right) \times 100 \%$

where $P_{B}^{\min }$ and $P_{B}^{\max }$ are the minimum and maximum BESS power $(\mathrm{kW})$, $P_{\text {dcha }}$ and $P_{\text {cha,t }}$ are the discharging and charging power of the battery (kW), $P_{d c h a}^{\min }$ and $P_{d c h a}^{\max }$ are the minimum and maximum discharging power $(\mathrm{kW}), P_{c h a}^{\min }$ and $P_{c h a}^{\max }$ are the minimum and maximum charging power $(\mathrm{kW}), E_{B}^{\min }$ and $E_{B}^{\max }$ are the minimum and maximum BESS energy (kWh), SOC ${ }^{\min }$ and $S O C^{\max }$ are the minimum and maximum state of charge of the BESS, $\eta_{d c h a}$ and $\eta_{\text {cha }}$ are the discharging and charging efficiency of the battery, $E_{B, t}$ is the BESS energy at t-th time (kWh), $b_{t}$ is the BESS operating variable at t-th time [' 0 ' discharge, ' 1 ' charge], $S O C_{t}$ is the BESS state of charge at $\mathrm{t}$-th time.

$P_{c h a}^{m a x}$ is used as a threshold value to differentiate between load demand interval (usually at terminal) and high demand interval (usually while cruising). When the load demand is less than the maximum charging power $\left(P_{L, t} \leq P_{c h a}^{\max }\right)$, the ferry is at the low demand interval (at terminal). When the load demand is more than the maximum charging power $\left(P_{L, t}>P_{c h a}^{\max }\right)$, the ferry in at the high demand interval (cruising).

\section{- GHG emissions constraints}

The DGs are the GHG emissions source in the system. Therefore, the emissions constraints are designed to ensure that the emissions at terminal (berth) are always zero. The berth and cruising emissions are calculated based on Table 2 .

$e_{\text {berth }}=0 ; P_{L, t} \leq P_{c h a}^{\max }$

$e_{\text {cruising }} \leq e_{\max } ; P_{L, t}>P_{\text {cha }}^{\max }$

where $e_{\max }$ is the maximum emissions limit in $\mathrm{g} / \mathrm{kWh}$ (when both 
generators are operated at their maximum capacity), $e_{\text {berth }}$ and $e_{\text {cruising }}$ are the emissions at berth (terminal) and while cruising calculated by the following equations:

$e_{\text {berth }}=e_{\mathrm{CO}_{2}}^{\text {berth }}+e_{\mathrm{SO}_{X}}^{\text {berth }}+e_{\mathrm{NO}_{X}}^{\text {berth }}$

$e_{\text {cruising }}=e_{\mathrm{CO}_{2}}^{\text {cruising }}+e_{\mathrm{SO}_{X}}^{\text {cruising }}+e_{\mathrm{NO}_{X}}^{\text {cruising }}$

where $e_{\mathrm{CO}_{2}}^{\text {berth }}, e_{\mathrm{SO}_{X}}^{\text {berth }}$ and $e_{\mathrm{NO}}^{\text {berth }}$ are the $\mathrm{CO}_{2}, \mathrm{SO}_{X}$ and $\mathrm{NO}_{X}$ emissions at berth $(\mathrm{g} / \mathrm{kWh})$ and $e_{\mathrm{CO}_{2}}^{\text {cruising }}, e_{\mathrm{SO}_{X}}^{\text {cruising }}, e_{\mathrm{NO}}^{\text {cruising }}$ are the the $\mathrm{CO}_{2}, \mathrm{SO}_{X}$ and $\mathrm{NO}_{X}$ emissions while cruising $(\mathrm{g} / \mathrm{kWh})$.

\section{- Blackout prevention constraints}

Blackout prevention constraints are important to ensure reliability and security of the power system. The differences between the maximum power at generation side (including BESS) and the maximum load power must be more than or equal to zero.

$N \times g_{t n} \times P_{n}^{\max }+P_{B, t}^{\max }-P_{L, t} \geq 0$

where $P_{B, t}^{\max }$ is the maximum power of the BESS at t-th time $(\mathrm{kW})$ and it can be calculated by:

$P_{B, t}^{\max }=P_{d c h a}^{\max } \times\left(1-b_{t}\right)-P_{\text {cha }}^{\max } \times b_{t}$

The GWO algorithm for fuel consumption minimization of the shorthaul hybrid ferry has been implemented using MATLAB software. The GWO parameter values used during the simulation are maximum number of iterations $=1000$, number of search agents $=30$ and problem dimensions is equal to three. The flowchart of fuel consumption minimization using GWO is shown in Fig. 5.

\section{System modeling}

\subsection{Loads}

Usually the main load of a fixed route short haul ferry is the propulsion load while the service load takes a small portion of the total load. To simplify the model representation of the propulsion load, a variable resistance is used to represent the propulsion load. The load profile in $\mathrm{kW}$ is converted into a pure resistance values by the following equation:

$R=\frac{V_{b u s}^{2}}{P_{L}}$

where $V_{b u s}$ is the measured voltage at the bus and $P_{L}$ is the load power in $\mathrm{kW}$.

The total resistance value of the load profile is distributed to the main load and secondary load. The main load is considered as a propulsion load as it is considered the largest load while the secondary load is the service load. The resistance distribution of the load profile can be calculated by:

$R_{\text {prop }}=\lambda_{p} R$

$R_{\text {serv }}=\lambda_{s} R$

where $R_{\text {prop }}$ is the propulsion load resistance $(\Omega), R_{\text {serv }}$ is the service load resistance $(\Omega), \lambda_{\text {prop }}$ is the ratio of propulsion load and $\lambda_{\text {serv }}$ is the ratio of service load.

\subsection{Diesel Gen-Set (DGs)}

Diesel generators are considered as the main power source in the vessel. The diesel generator specifications are used based on Cummins DG specifications [48]. Table 3 provides the technical specification of the existing DGs.

The synchronous round rotor machine is used to model the diesel generator. For simulation, transient and sub transient parameter values are converted to fundamental per-unit parameters based on classical definitions.

The synchronous machine equations are expressed with respect to a rotating reference frame defined by the equation

$\theta e(t)=N_{p} \theta r(t)$

where $\theta_{\mathrm{e}}$ is the electrical angle, $N_{p}$ is the number of pole pairs, and $\theta_{\mathrm{r}}$ is the rotor angle.

The model of the diesel gen-set contains sub-models for the synchronous round rotor machine, speed governor and automatic voltage regulator. The governor, which comes as an electronic controller, regulate the diesel fuel supply to the engine which in turn control the rotational speed of the rotor $(\omega)$. The controller lets the frequency vary

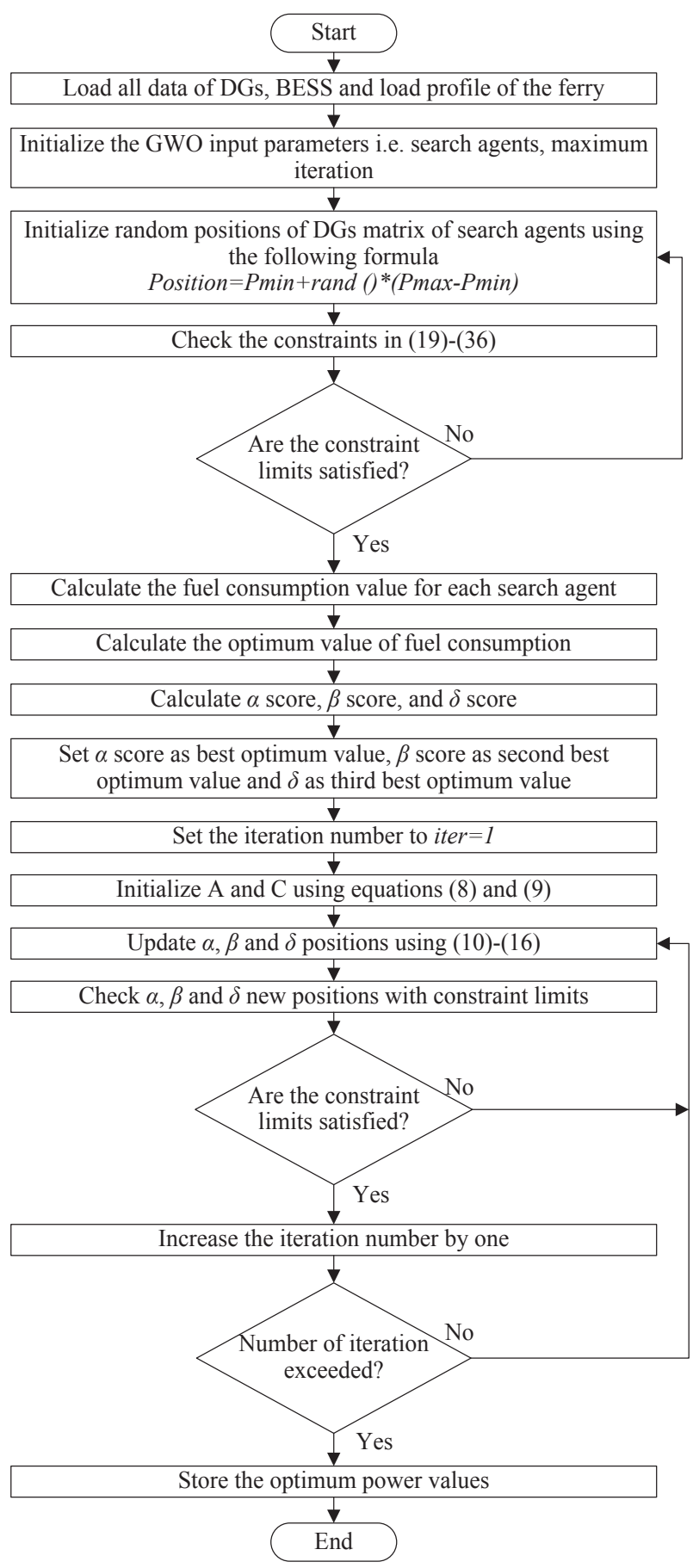

Fig. 5. Flowchart of the power management strategy using GWO. 
Table 3

Diesel set specifications (summarized from [48]).

\begin{tabular}{llll}
\hline $\begin{array}{l}\text { Generator specifications } \\
\text { Model }\end{array}$ & C350 D6 & $\begin{array}{l}\text { Output power (kWe) } \\
\text { Output voltage (V) }\end{array}$ & 320 \\
S rating (kVA) & 400 & Phase & 3 \\
$\begin{array}{l}\text { P.f } \\
\text { Frequency (Hz) }\end{array}$ & 0.8 & & \\
$\begin{array}{l}\text { Engine specifications } \\
\text { Manufacturer }\end{array}$ & 60 & Model & NTA855 G3 \\
$\begin{array}{l}\text { Output - Prime (kWm) } \\
\text { Rated speed (rpm) }\end{array}$ & 358 & No. of cylinders & 6 in- line \\
& 1800 & & \\
\hline
\end{tabular}

in proportion to the active power $(P)$ of the load. The block diagrams of the droop speed controller is shown in Fig. 6.

\subsection{Battery sizing}

A BESS is used to reduce the diesel fuel consumption by supplying electricity to loads at low demand conditions at the terminal. The size capacity of the BESS is calculated by the following equation:

$E_{B}=N_{S} E_{T, \max }+0.2 \times N_{S} E_{T, \max }$

$E_{B}=N_{S} E_{T, \max }(1+0.2)$

where $E_{B}$ is the energy capacity of the battery pack in kWh, $N_{S}$ is the number of stops per one round-trip and $E_{T, \max }$ is the highest energy at one terminal in $\mathrm{kWh}$. The constant 0.2 represents the minimum SOC (80\% DOD) recommended from several marine battery manufacturers to maintain a reasonable cycle life of the battery $[61,62]$. Looking into different marine battery manufacturers, the battery module specification shown in Table 4 is considered in this study [61].

The number of battery modules in the BESS is determined based on the total energy required. In other words, the total energy capacity of the battery pack must be larger than or equal to the maximum energy required by the load. The battery modules can be arranged into several different configurations depending on the voltage level and the current capacity required. The two common battery configurations are parallel and series configurations as shown in Fig. 7. The parallel configuration provides more current capacity (Ah) than series configuration. Hence, parallel configuration is used for high current low voltage applications while series configuration is applicable for low current and high voltage applications.

\subsection{Inverter}

An inverter is used to convert the DC voltage from the DC bus to AC voltage at the required voltage level and frequency to drive the propulsion motors. A schematic diagram of the inverter is shown in Fig. 8.

The power, resistance, and currents are defined by

$P_{A C}=-v_{a} i_{a}-v_{b} i_{b}-v_{c} i_{c}$

$R_{D C}=\frac{v_{D C}^{2}}{P_{A C}+P_{\text {fixed }}}$

$i=\frac{V_{D C}}{R_{D C}}$

where $i_{a}, i_{b}, i_{c}$ are the respective AC phase currents flowing into the inverter, $P_{A C}$ is the power output on the AC side, $P_{f i x e d}$ is the fixed power loss that is specified on the block, $R_{D C}$ is the resistance on the DC side, and $i$ is the current flowing from the positive to the negative terminals of the inverter.

The ratio of $V_{r m s}$ to $V_{d c}$ is chosen to be 0.7797 based on the following equation [63].

$V_{r m s}($ line-line $)=\frac{\sqrt{6}}{\pi} V_{D C}$

\subsection{Rectifier}

The rectifier is used to convert three-phase AC voltage to DC voltage. The average rectifier model produce a full-wave output using the six-pulse rectifier. The schematic diagram of the six-pulse rectifier is shown in Fig. 9.

The output voltage of the rectifier $V_{d c}$ is:

$V_{D C}=\frac{3 \sqrt{2}}{\pi} \times V_{R M S}$

where

$V_{R M S}=\sqrt{\frac{\left(v_{a}-v_{b}\right)^{2}+\left(v_{b}-v_{c}\right)^{2}+\left(v_{c}-v_{a}\right)^{2}}{3}}$

$v_{a}, v_{b}, v_{c}$ are the respective AC input phase voltages.

The power into the rectifier is defined in the following equation:

$P_{A C}=P_{\text {loss }}+P_{D C}$

The DC power output from the rectifier is:

$P_{D C}=P_{A C}-P_{\text {loss }}$

The power loss drawn by the rectifier is:

$P_{\text {loss }}=\frac{V_{\text {Rated }}}{R_{\text {fixed }}}$

where $V_{\text {Rated }}$ is the rated voltage at the AC side and $R_{\text {fixed }}$ is the phase series resistance in an equivalent wye connected load.

\section{6. $D C-D C$ converter}

To incorporate the BESS to the HPS, a DC-DC converter is normally used. A behavioural model of a bidirectional DC-DC converter is used to regulate and convert the DC voltage of the battery from one voltage level to another. In addition, the converter is used to regulate and stabilize voltage at the dc bus. The output voltage of the converter is defined by:

$v=v_{\text {ref }}-i_{\text {load }} D+i_{\text {load }} R_{\text {out }}$

where $v_{r e f}$ is the DC bus voltage set point, and $D$ is the value for the output voltage droop with an output current parameter.

The block parameters of the DC-DC converter are shown in Table 5.

\section{Case study}

\subsection{Description of ferry and voyage}

Bruny Island is located off the south-eastern coast of Tasmania, Australia, and encompasses approximately 363 square kilometers; it is considered a popular tourist attraction. Access to Bruny Island is available by two ferries, namely Mirambeena and Bowen. In this paper, Bowen ferry is selected as the case study. Bowen ferry operates between Kettring (terminal 1) and Bruny Island (terminal 2) as shown in Fig. 10.

The specifications of Bowen ferry are given in Table 6 . The ferry operates six days a week and performs 42 round trips per week ( 7 round trips per day) during the peak period.

The single-line diagram of the existing AC power system is shown in Fig. 11. The model includes two DGs, propulsion loads and a service load.

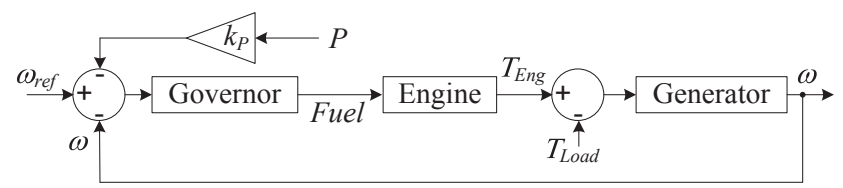

Fig. 6. Block diagram of the speed controller of the DG [60]. 
Table 4

Battery module specifications.

\begin{tabular}{|c|c|c|c|c|c|c|c|c|c|c|}
\hline \multirow[t]{2}{*}{ Manufacturer } & \multirow[t]{2}{*}{ Model } & \multirow[t]{2}{*}{ Cell chemistry } & \multirow{2}{*}{$\begin{array}{l}\text { Energy } \\
(\mathrm{kWh})\end{array}$} & \multirow[t]{2}{*}{ Capacity (Ah) } & \multicolumn{3}{|c|}{ Voltage range $(\mathrm{V})$} & \multirow{2}{*}{$\begin{array}{l}\text { Max. discharge power } \\
-P_{d c h a}^{\max }(\mathrm{kW})\end{array}$} & \multirow{2}{*}{$\begin{array}{l}\text { Max. charging power } P_{c h a}^{\max } \\
(\mathrm{kW})\end{array}$} & \multirow{2}{*}{$\begin{array}{l}\text { Cycle Life at } 80 \% \\
\text { DoD }\end{array}$} \\
\hline & & & & & $\operatorname{Max}$ & Nominal & Min & & & \\
\hline PBES & Power 65 & NMC & 6.5 & 75 & 100 & 88.8 & 77 & 45 & 22.5 & 15,000 \\
\hline
\end{tabular}

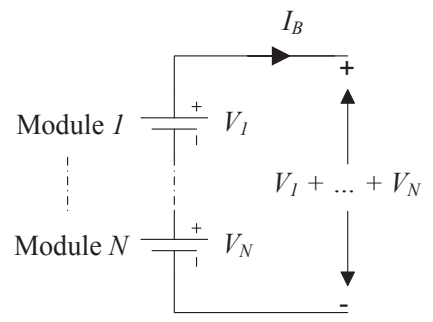

(a)

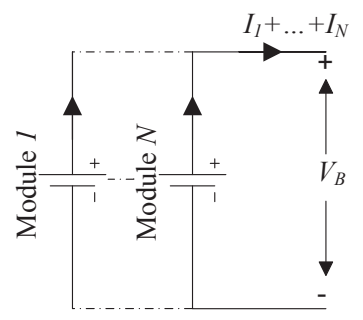

(b)
Fig. 7. Battery module configurations: (a) Series configuration and (b) Parallel configuration.

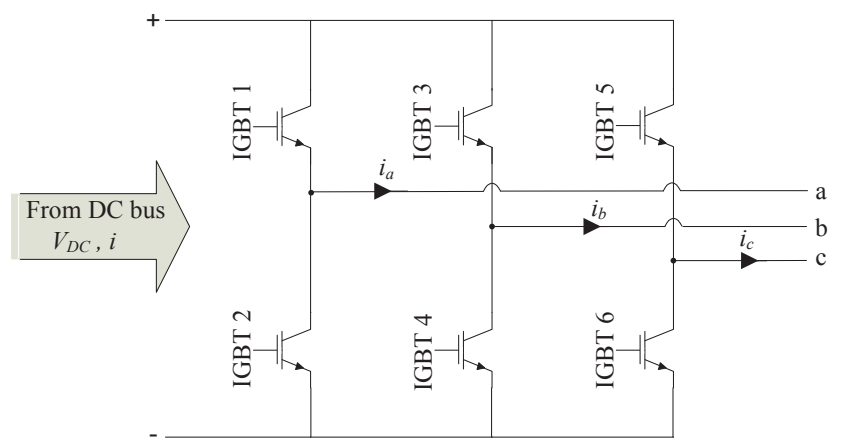

Fig. 8. The equivalent circuit of the average inverter.

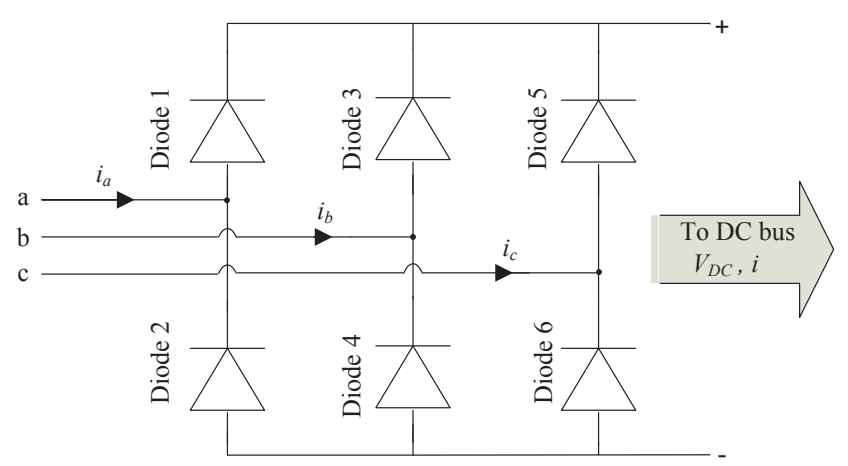

Fig. 9. The equivalent circuit of the average rectifier.

Table 5

DC-DC converter block parameters.

\begin{tabular}{ll} 
DC bus voltage reference (Vref) & $520 \mathrm{~V}$ \\
Related output power & $200 \mathrm{~kW}$ \\
Droop parameterization & By voltage droop with output \\
& current \\
Output voltage droop with output current & 0.05 \\
Power direction & Unidirectional \\
Maximum expected supply-side current & $135 \mathrm{~A}$ \\
\hline
\end{tabular}

The measured load profile of the ferry is shown in Fig. 12.

According to the measured load profile, the ferry requires a total energy of $200.175 \mathrm{kWh}$ to complete one round trip. The energy consumption for each operational condition is shown in Fig. 13. The energy consumption at terminal, which also covers the manoeuvring period, occurs below $67 \mathrm{~kW}$, while energy consumption at cruising covering the manoeuvring period occurs above $67 \mathrm{~kW}$. As this ferry is a single deck ferry, the wind effect is negligible. In addition, as the ferry is operated within an area enclosed by land (as this ferry is a short-haul ferry which operates for short distances only), the wave effect is also negligible. In the first cruising period, the ferry was fully loaded with the maximum vehicles capacity (30 cars). In the second cruising period, the load on the ferry was less than the first cruising period. As results, in the second cruising period, the thrusters require less power.

As mentioned in Section 4.1, the load profile in $\mathrm{kW}$ is converted into a pure resistance value. The ratio of propulsion load to the total load $\lambda_{p}$ is set to 0.9 while the ratio of service load to the total load $\lambda_{s}$ is set to 0.1. Fig. 14 shows the voltage, current and resistance value of each propulsion load.

\subsection{Proposed HPS with DC distribution}

As the ferry terminal at Kettering is close to a residential area, emissions and noise produced by the on-board DGs is a concern. The emissions produced by the DGs cause direct impacts to the heath of people living near the ferry terminal. To overcome these issues, a BESS based HPS solution is proposed in this paper, where the engines are turned off when the ferry is in and around the terminals. For this purpose, the DC distribution system is proposed to replace the existing AC distribution system of the ferry. The single-line diagram of the proposed HPS with DC distribution is shown in Fig. 15. The model includes two DGs, BESS, DC-DC converter, rectifiers, inverter, propulsion loads and service load.

The battery module specifications used for this case study are provided in Table 4. According to Figs. 12 and 13, the maximum energy consumption $\left(E_{T, \max }\right)$ occurred at terminal 2 . Therefore, $E_{T, \max }$ is equal to the total energy consumption at terminal 2 which is $11.42 \mathrm{kWh}$.

By using Eq. (42), the total energy required from the BESS at berth is found to be $27.408 \mathrm{kWh}$. However, as there are two cruising periods in the one round trip, the battery pack can be charged in each cruising period. Therefore, the energy required from the battery pack can be reduced to half. As a result, the size capacity of the BESS can be $13.704 \mathrm{kWh}$. This will significantly reduce the initial cost of the battery system.

As the voltage at the DC bus is $520 \mathrm{~V}$ and the required energy capacity is low, the suitable configuration for the battery modules is the series configuration. Therefore, based on battery specifications provided in Table 4, three battery modules are considered to form the battery pack as shown in Fig. 16.

Based on the above, the specifications of the BESS pack are shown in Table 7.

\subsection{Fuel consumption indicator}

Based on Eq. (2), the complete SFOC curve of the ferry's main engine is presented in Fig. 17.

DGs are generally considered as the main source of power in a hybrid ship power system. They provide continues supply which maintains the voltage and meets the average load demand. Whenever there are fluctuations in the load the BESS is used. To effectively run the 


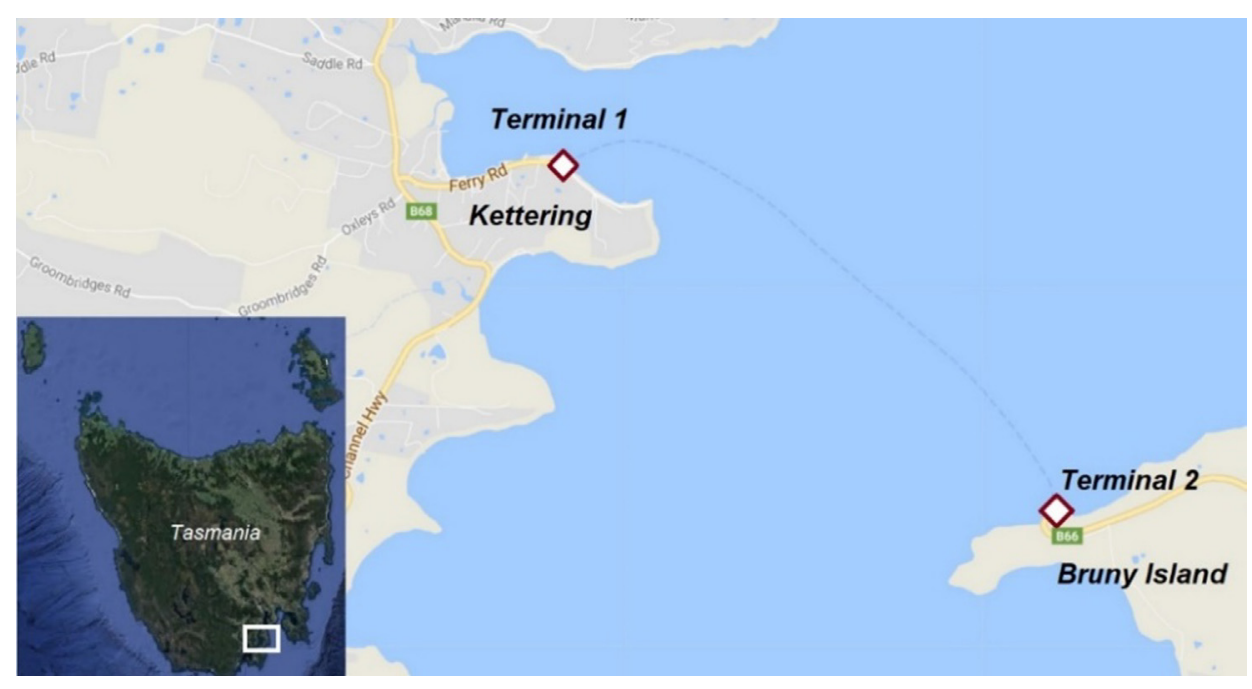

Fig. 10. The examined ferry route.

Table 6

Ferry specifications.

\begin{tabular}{llllll}
\hline \multicolumn{2}{l}{ Bowen ferry specifications and voyage descriptions } & & \\
\hline Ferry capacity & $\begin{array}{l}30 \\
\text { vehicles }\end{array}$ & $\begin{array}{l}\text { Service } \\
\text { speed }\end{array}$ & 7 knots & Length & $35 \mathrm{~m}$ \\
Powering & $\begin{array}{l}2 \times 400 \\
\mathrm{kVA}\end{array}$ & Fuel type & Diesel & Breadth & $15 \mathrm{~m}$ \\
Travel distance & $\begin{array}{l}(320 \mathrm{~kW}) \\
6.2 \mathrm{~km} \\
\text { (round }-\end{array}$ & $\begin{array}{l}\text { Travel } \\
\text { duration }\end{array}$ & $\begin{array}{l}60 \mathrm{~min} \\
\text { (round } \\
\text { trip) }\end{array}$ & Propulsion & $\begin{array}{l}2 \times \text { Azimuth } \\
\text { thrusters }\end{array}$ \\
& & & & \\
\hline
\end{tabular}

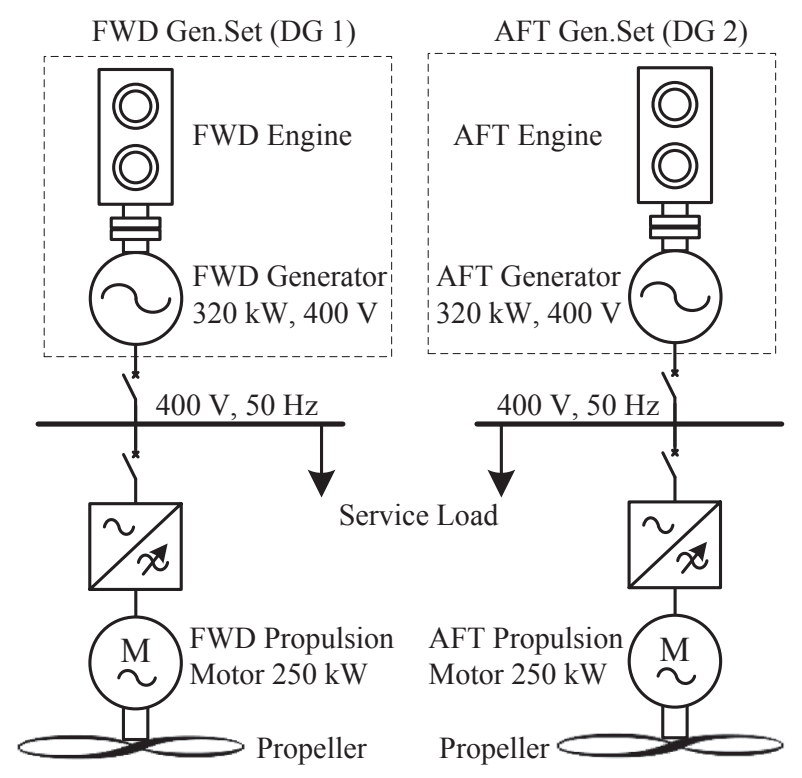

Fig. 11. Single-line diagram of the existing ferry power system.

diesel engine, it should operate only at its high operational efficiency range, that is from $60 \%$ to $100 \%$ of its rated power as shown in Fig. 17. This attributes to lowering the fuel consumption of the diesel engine. As shown in Fig. 17, the optimal SFOC is $0.266 \mathrm{~L} / \mathrm{kWh}$ at $86.5 \%$ engine load. According to the proposed PMS, when the load demand is low, the BESS is operated as the main power supply source. This helps to lower the operating hours of the DGs which reduces fuel consumption, emissions and maintenance costs of the diesel generators, and increase fuel savings and the life time of the diesel generator. In addition, operating the BESS as the main power supply at low load demand (when ferry is at terminal) eliminates the noise from DGs.

Based on the measured power consumption profile and by using Eqs. (2)-(5), the SFOC and fuel consumption for (DG 1) and (DG 2) are calculated and shown in Fig. 18.

The total fuel consumption of the ferry is $57.7 \mathrm{~L}$, which consists of fuel consumption of DG 1 at $31.5 \mathrm{~L}$ and the fuel consumption of DG 2 at 26.6 L. Table 8 shows the fuel consumption for both engines at each terminal and at different operating conditions.

In order to calculate the fuel cost, the average price of diesel fuel of AUD\$ 1.405 per liter in Tasmania is used (provided by the Australian Institute of Petroleum). Hence, the total fuel cost of one round trip is AUD\$ 81.07.

\subsection{Emissions indicator}

In order to estimate the emissions produced by the ferry, measured energy consumption at berth and while cruising for the existing ferry system is used. Table 9 shows the $\mathrm{CO}_{2}, \mathrm{SO}_{\mathrm{X}}$ and $\mathrm{NO}_{\mathrm{X}}$ emissions from the ferry per one round trip.

\subsection{Ferry PMS parameters}

The values used in the PMS of the proposed hybrid system are given in Table 10.

\section{Simulation results and analysis}

The ferry takes $60 \mathrm{~min}$ to perform a complete round trip. The measured load profile is inserted into the MATLAB Simulink ${ }^{\circledast}$ simulation platform. The simulation of the existing AC system is performed to validate the model and estimate the SFOC, fuel consumption and emissions. Then, the HPS is simulated for same ferry operation with different PMSs to investigate the reduction of SFOC, fuel consumption and emissions compared with existing AC system. In addition, a performance comparison between classical and meta-heuristic PMSs used for the short-haul hybrid ferry is conducted.

\subsection{Existing AC system}

The existing AC system is modeled in Simulink. The measured load profile and the fuel consumption of the ferry are used to validate the accuracy of the simulation model.

From Section 5.3, the fuel consumption of the ferry based on the measured load profile is found to be $57.7 \mathrm{~L}$. The simulation results of 


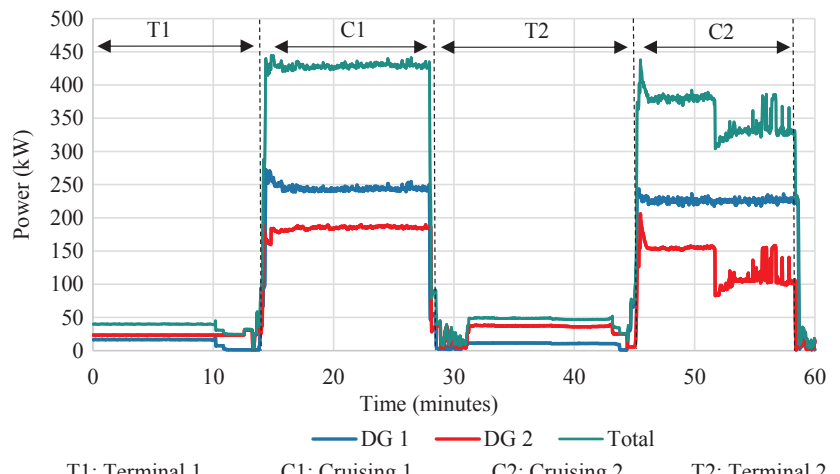

Fig. 12. The measured load profile of Bowen Ferry.

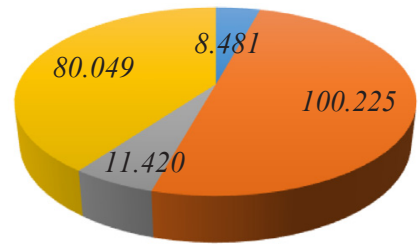

Energy at Terminal $1(\mathrm{kWh}) \quad$ Energy at Cruising $1(\mathrm{kWh})$

Energy at Terminal $2(\mathrm{kWh}) \quad$ Energy at Cruising $2(\mathrm{kWh})$

Fig. 13. The measured energy consumption for each operating condition.
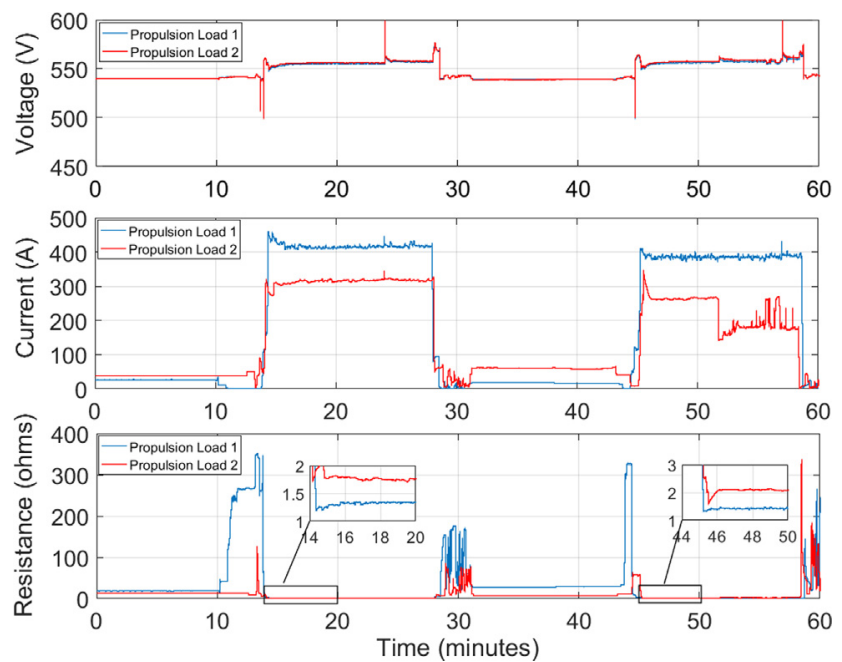

Fig. 14. Voltage, current and resistance of propulsion load 1 and 2.

the model provided a total fuel consumption of $59.17 \mathrm{~L}$ as shown in Table 11. The error percentage between the calculated fuel consumption based on actual data and the simulated fuel consumption is $2.484 \%$. This percentage validated the accuracy of the simulation model.

The simulation outputs of generated powers from the two existing DGs and the load power are depicted in Fig. 19. The simulation results of the fuel consumption and generated powers show acceptable agreement between the measured load data and the simulated load profile from the model. This validated that the model is accurate with accuracy percentage of $97.516 \%$.

As can be seen from Fig. 19, both DGs are operated all the time. From 0 to $14 \mathrm{~min}$ and 30 to $45 \mathrm{~min}$, the ferry is berthed at the terminal. The two DGs are operated to supply the load. In both periods, each DG is operated in the low operational efficiency range where each diesel engine generates approximately $20 \mathrm{~kW}(6.5 \%$ of the engine rated load) in the period from 0 to $14 \mathrm{~min}$ and $25 \mathrm{~kW}$ ( $8.2 \%$ of engine rated load) in

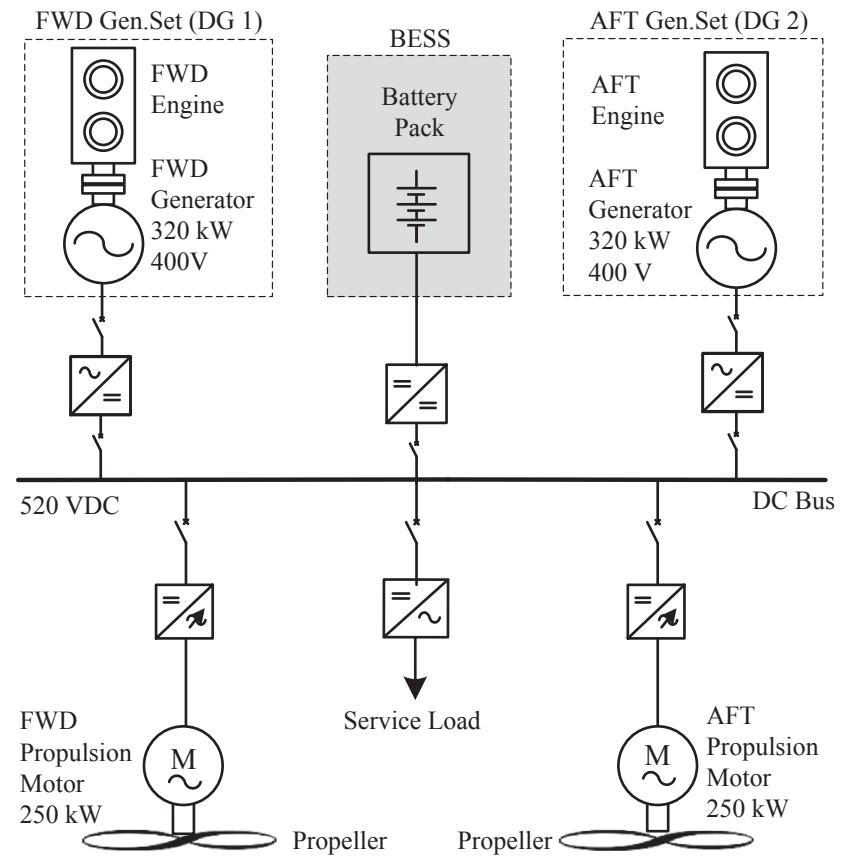

Fig. 15. The single-line diagram of the proposed HPS.

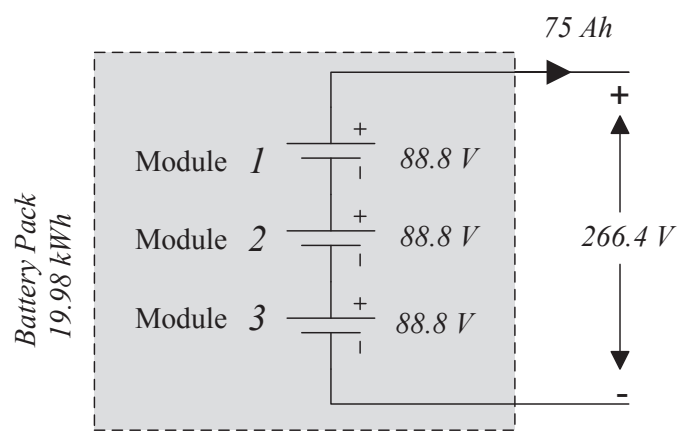

Fig. 16. Battery modules configuration in the battery pack.

Table 7

BESS parameters.

\begin{tabular}{|c|c|c|c|c|c|c|c|}
\hline \multirow{2}{*}{$\begin{array}{l}\text { Nominal } \\
\text { voltage } \\
\text { (V) }\end{array}$} & \multirow{2}{*}{$\begin{array}{l}\text { Rated } \\
\text { current } \\
\text { (Ah) }\end{array}$} & \multirow{2}{*}{$\begin{array}{l}\text { Energy } \\
\text { Capacity } \\
E_{B}(\mathrm{kWh})\end{array}$} & \multicolumn{2}{|c|}{ SOC (\%) } & \multirow{2}{*}{$\begin{array}{l}\text { Max. } \\
\text { discharge } \\
\text { power } \\
P_{d c h a}^{\max }(\mathrm{kW})\end{array}$} & \multirow{2}{*}{$\begin{array}{l}\text { Max. } \\
\text { charging } \\
\text { power } \\
P_{\text {cha }}^{\max }(\mathrm{kW})\end{array}$} & \multirow{2}{*}{$\begin{array}{l}\text { Charge/ } \\
\text { discharge } \\
\text { efficiency } \\
(\%)\end{array}$} \\
\hline & & & Min & Max & & & \\
\hline 266.4 & 75 & 19.98 & 20 & 100 & 135 & 67.5 & 98 \\
\hline
\end{tabular}

the period from 30 to $45 \mathrm{~min}$.

The simulation output of the fuel consumption when the ferry is berthed both at terminal 1 and $2\left(F C_{\text {berth }}\right)$ is $8.43 \mathrm{~L}$. Table 12 shows the fuel consumption obtained from the simulation model.

At the first cruising period from 15 to $29 \mathrm{~min}$, DG 1 and DG 2 are operated at approximately $78 \%$ and $57 \%$ of rated engine load respectively. The corresponding fuel consumption during cruising period 1 $\left(F C_{\text {cruising } 1}\right)$ is about $27.83 \mathrm{~L}$. At the second cruising period from 46 to $59 \mathrm{~min}$, DG 1 operates at approximately $230 \mathrm{~kW}(71.8 \%$ of rated engine load) while DG 2 operates from $155 \mathrm{~kW}$ to $107 \mathrm{~kW}$ ( $48.4 \%$ to $33.4 \%$ of rated engine load). The corresponding fuel consumption during cruising period $2\left(F C_{\text {cruising } 2}\right)$ is about $22.92 \mathrm{~L}$. As a result, the total fuel consumption of both DGs while cruising $\left(F C_{\text {cruising }}\right)$ is $50.57 \mathrm{~L}$. Based on the simulation result, the fuel cost of one round trip is AUD\$ 83.13.

From simulation results, the total energy required for one round trip 


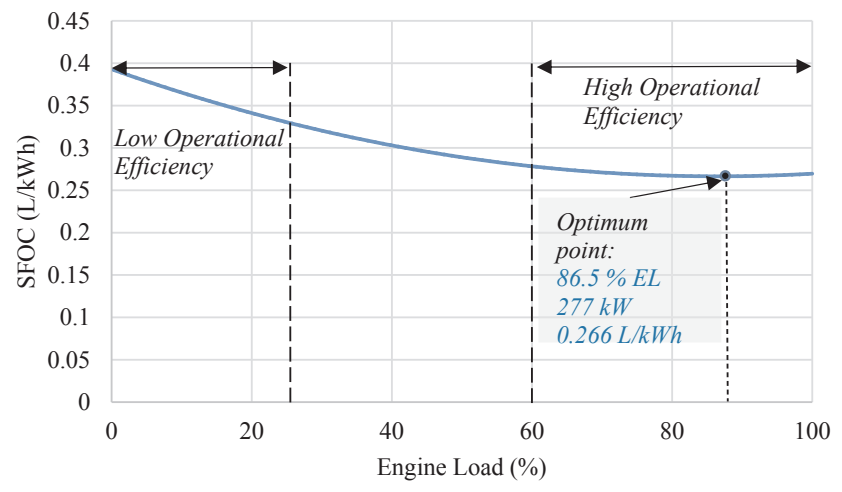

Fig. 17. The estimated SFOC curve of the existing diesel engine.

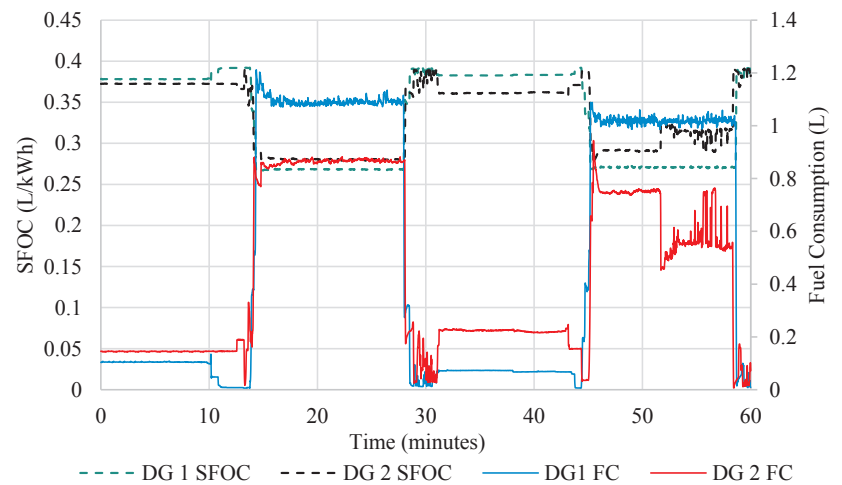

Fig. 18. SFOC and fuel consumption (FC) of DG 1 and DG2.

is $202.903 \mathrm{kWh}$ which consists of $20.98 \mathrm{kWh}$ at berth and $181.93 \mathrm{kWh}$ while cruising. The simulation results show acceptable agreement between the measured energy consumption, which is $200.175 \mathrm{kWh}$, and the simulated energy consumption, which is $202.903 \mathrm{kWh}$.

Based on the energy consumption output from the simulation model and the equations in Table 2, the $\mathrm{CO}_{2}, \mathrm{SO}_{\mathrm{X}}$ and $\mathrm{NO}_{\mathrm{X}}$ emissions are calculated for both DGs and depicted in Table 13.

\subsection{HPS using Rule-Based method (HPS-RB)}

The validated Simulink model of the existing AC system is then modified to the HPS with DC distribution as depicted in Fig. 15. As the proposed system consists of multi energy sources (DGs and BESS), a PMS is essential to ensure optimal sharing and operation of the system. In this sub-section, the proposed HPS is simulated with RB method as the PMS. The simulation output of the power generated by DG 1 and DG 2, load power and BESS power are shown in Fig. 20 and the SOC of the BESS is shown in Fig. 21.

At the period from 0 to $14 \mathrm{~min}$ and 30 to $45 \mathrm{~min}$, when the ferry is at the terminal, the load power is less than the lower load power boundary of the controller as shown in Fig. 20 and the battery SOC is at the upper boundary as shown in Fig. 21. Therefore, the controller disconnected both DGs and discharged the battery in order to supply the load demand. This provides quieter operation and zero emissions as the ferry is
Table 9

The emissions produced by the ferry in one round trip.

\begin{tabular}{lllll}
\hline & $\begin{array}{l}\mathrm{CO}_{2} \text { emissions, } \\
\mathrm{E}_{\mathrm{CO} 2}(\mathrm{~g} / \text { trip })\end{array}$ & $\begin{array}{l}\mathrm{SO}_{\mathrm{x}} \text { emissions, } \\
\mathrm{E}_{\mathrm{SOX}}(\mathrm{g} / \text { trip })\end{array}$ & $\begin{array}{l}\mathrm{NO}_{\mathrm{x}} \text { emissions, } \\
\mathrm{E}_{\mathrm{NOx}}(\mathrm{g} / \text { trip })\end{array}$ & $\begin{array}{l}\text { Total emissions } \\
(\mathrm{g} / \text { trip })\end{array}$ \\
\hline Berth & 13572.48 & 230.85 & 238.81 & 14042.15 \\
Cruising & 111769.67 & 1892.87 & 2704.11 & 116366.65 \\
Total & 125342.16 & 2123.73 & 2942.92 & 130408.80 \\
\hline
\end{tabular}

Table 10

PMS parameters of the proposed hybrid ferry.

\begin{tabular}{|c|c|c|c|c|c|}
\hline \multicolumn{6}{|c|}{ PMS parameters } \\
\hline SOC Cinitial & $100 \%$ & $P_{d c h a}^{\min }$ & $0 \mathrm{~kW}$ & $P_{n}^{\min }$ & $0 \mathrm{~kW}$ \\
\hline $\operatorname{SOC}^{\max }$ & $100 \%$ & $P_{d c h a}^{m a x}$ & $135 \mathrm{~kW}$ & $P_{n}^{\max }$ & $320 \mathrm{~kW}$ \\
\hline SOC high & $90 \%$ & $P_{c h a}^{\min }$ & 0 & $N$ & 2 \\
\hline$S O C^{\min }$ & $20 \%$ & $P_{c h a}^{\max }$ & $67 \mathrm{~kW}$ & $P_{L}^{\max }$ & $640 \mathrm{~kW}$ \\
\hline$P_{B}^{\min }$ & $-135 \mathrm{~kW}$ & $E_{B}^{\min }$ & $3.996 \mathrm{kWh}$ & $P_{L}^{a v e}$ & $320 \mathrm{~kW}$ \\
\hline$P_{B}^{\max }$ & $67.5 \mathrm{~kW}$ & $E_{B}^{\max }$ & $19.98 \mathrm{kWh}$ & $P_{L}^{\min }$ & $67 \mathrm{~kW}$ \\
\hline$\eta_{\text {cha }}$ & 0.98 & $\eta_{d c h a}$ & 0.98 & $N_{S}$ & 2 \\
\hline
\end{tabular}

in the terminal and close to a residential area. As both DGs are disconnected at this period, the $F C_{\text {berth }}$ is equal to zero.

At the first cruising period from 15 to $29 \mathrm{~min}$, both DGs are running at their high operational efficiency range by generating $256 \mathrm{~kW}$ each. At this period, the battery SOC is at the low boundary limit as shown in Fig. 21. Therefore, the battery is in the charging mode. At $t=24$, the battery starts to operate in standby mode as the battery's SOC reaches the allowable limit after the battery is being charged. As a result, the fuel consumption of the first cruising period $F C_{\text {cruising } 1}$ is $30.74 \mathrm{~L}$. At the second cruising period from 46 to $59 \mathrm{~min}$, the controller connects both DGs and charges the battery as the load demand is increased and the battery SOC reaches the lower boundary. Each DG is operated at their high operational efficiency range by generating $236 \mathrm{~kW}$ to $210 \mathrm{~kW}$ (73.7\%-65.6\% of rated engine load). The controller is responsible for charging the battery only when both DGs are running at their high efficiency range and the battery SOC is at the lower boundary. Therefore, the battery is still charging, as the battery SOC has not reached a certain value set by the controller. At $t=58$, the battery SOC reaches the allowable limit as shown in Fig. 21. Due to that, the controller switches the battery to the standby mode. As a result, the fuel consumption in the second cruising period $F C_{\text {cruising }}$ is 26.7 . Therefore, the total fuel consumption of both DGs while cruising $\left(F C_{\text {cruising }}\right.$ ) is $57.45 \mathrm{~L}$. Overall, the total $F C_{\text {total }}$ for one round trip (60 min) of the HPS using RB (HPS-RB) is $57.45 \mathrm{~L}$. Table 14 shows the fuel consumption obtained from the simulation model of HPS-RB.

The controller successfully achieves a good power sharing among the two DGs and BESS by operating both engines at their high efficiency range and discharges the battery at a low demand period when the ferry is at the terminal in order to reduce the fuel consumption and eliminate noise and emissions. However, as such classical PMS is set based on predetermined conditions, the controller equally shares the power between DGs and runs them at their high operational range rather than optimal operation point. In addition, it is recommended to charge the battery at a lower charging rate in order to keep the power generated from DGs at

Table 8

The fuel consumption of each DG and total fuel consumption of the existing ferry.

\begin{tabular}{llllllll}
\hline & \multicolumn{2}{l}{ Fuel consumption at berth (L) } & & \multicolumn{2}{c}{ Fuel consumption while cruising (L) } & \multicolumn{2}{c}{$\begin{array}{l}\text { Total fuel consumption } \\
\text { for complete } \\
\text { round trip (L) }\end{array}$} \\
\cline { 2 - 7 } & At terminal 1 & At terminal 2 & At berth (T1 + T2) & While cruising 1 & While cruising 2 & While cruising (C1 + C2) \\
\hline DG 1 & 1.23 & 1.19 & 2.42 & 15.14 & 13.93 & 29.07 & \\
DG 2 & 2.16 & 3.30 & 5.46 & 12.06 & 8.71 & 20.77 & 59.83 \\
Total & 3.38 & 4.49 & 7.87 & 27.20 & 22.63 & 49.70 \\
\hline
\end{tabular}


Table 11

The comparison between the actual fuel consumption and the simulated fuel consumption.

\begin{tabular}{|c|c|c|c|}
\hline & $\begin{array}{l}\text { Fuel consumption } \\
\text { of DG } 1(\mathrm{~L})\end{array}$ & $\begin{array}{l}\text { Fuel consumption } \\
\text { of DG } 2(\mathrm{~L})\end{array}$ & $\begin{array}{l}\text { Total fuel } \\
\text { consumption (L) }\end{array}$ \\
\hline Calculated & 31.5 & 26.2 & 57.7 \\
\hline Simulated & 33.1 & 26.07 & 59.17 \\
\hline Total error (\%) & & & 2.484 \\
\hline
\end{tabular}

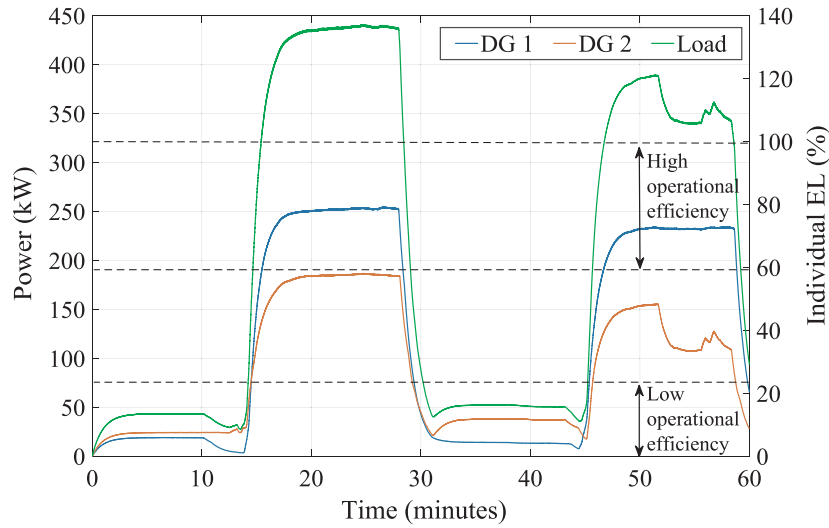

Fig. 19. Simulation output of power generated from diesel gen-sets.

the high operational range when the load demand decreases. For example, as shown in Fig. 21, the battery is turned to standby mode at $\mathrm{t}=25$. This results in a reduction from $80 \%$ to $67 \%$ of engine load which results in a corresponding increment in SFOC from $0.26720 \mathrm{~L} /$ $\mathrm{kWh}$ to $0.2734 \mathrm{~L} / \mathrm{kWh}$. Therefore, it is recommended to charge the battery at a uniform charging rate over the complete cruising period to keep the diesel engines operating at a higher engine load.

The total energy consumption is found to be $210.67 \mathrm{kWh}$ which is mainly occurred while cruising. The $\mathrm{CO}_{2}, \mathrm{SO}_{\mathrm{X}}$ and $\mathrm{NO}_{\mathrm{X}}$ emissions are calculated for both DGs and depicted in Table 15.

\subsection{HPS using grey wolf optimization (HPS-GWO)}

GWO is used to optimally manage the power sharing of the proposed HPS. As seen from the objective function of Eq. (17), the objective of optimization is to minimize the fuel consumption of DG1 and DG2 subject to the constraints explained in Eqs. (18)-(36). The main optimization parameters are the DG1 power, DG2 power and battery power. Optimizing those parameters will optimize the value of SFOC and results in reduction of fuel consumption. The power optimization results of the optimization parameters DG 1 power, DG 2 power and battery power are shown in Fig. 22 and the SOC of the BESS is shown in Fig. 23.

At the period from 0 to $14 \mathrm{~min}$ and 30 to $45 \mathrm{~min}$, when the ferry is at berth, the load power is less than the lower load power boundary and the battery SOC is at the upper boundary as shown in Fig. 23. Therefore, both DGs are off and the BESS is in the discharging mode. This provide quieter operation and zero emissions as the ferry is in the terminal and
Table 13

The emissions obtained from the simulation model of the AC system.

\begin{tabular}{llll}
\hline & $\begin{array}{l}\mathrm{CO}_{2} \text { emissions, } \mathrm{E}_{\mathrm{CO} 2} \\
(\mathrm{~g} / \text { trip })\end{array}$ & $\begin{array}{l}\mathrm{SO}_{\mathrm{x}} \text { emissions, } \mathrm{E}_{\mathrm{SOx}} \\
(\mathrm{g} / \text { trip })\end{array}$ & $\begin{array}{l}\mathrm{NO}_{\mathrm{x}} \text { emissions, } \\
(\mathrm{g} / \text { trip })\end{array}$ \\
\hline Berth & 14306.27 & 243.33 & 251.72 \\
Cruising & 112794.20 & 1910.22 & 2728.89 \\
Total & 127100.47 & 2153.56 & 2980.62 \\
\hline
\end{tabular}

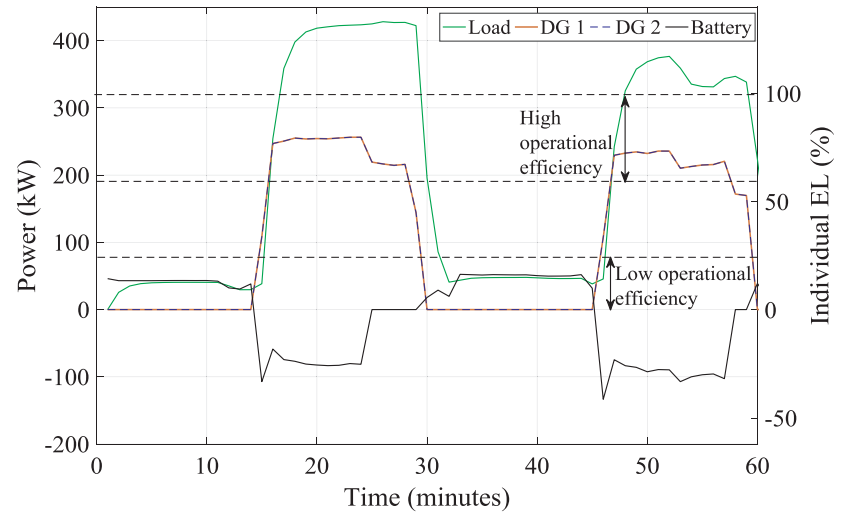

Fig. 20. The simulation output power of the proposed HPS-RB.

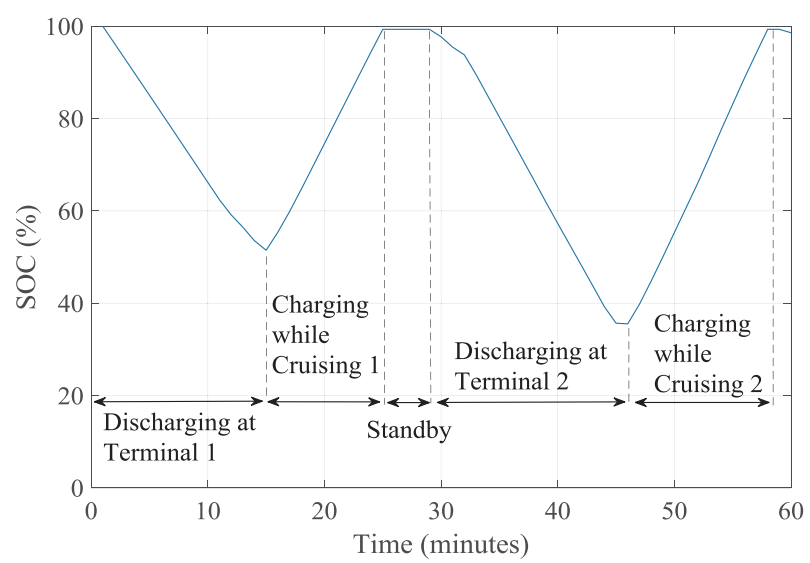

Fig. 21. The SOC of the BESS with HPS-RB.

Table 14

The fuel consumption obtained from HPS-RB.

\begin{tabular}{|c|c|c|c|c|c|}
\hline & \multirow{2}{*}{$\begin{array}{l}\text { Fuel } \\
\text { consumption } \\
\text { at berth (L) }\end{array}$} & \multicolumn{3}{|c|}{ Fuel consumption while cruising (L) } & \multirow{2}{*}{$\begin{array}{l}\text { Total fuel } \\
\text { consumption for } \\
\text { complete round } \\
\text { trip (L) }\end{array}$} \\
\hline & & $\begin{array}{l}\text { While } \\
\text { cruising } 1\end{array}$ & $\begin{array}{l}\text { While } \\
\text { cruising } 2\end{array}$ & $\begin{array}{l}\text { While } \\
\text { cruising } \\
(\mathrm{C} 1+\mathrm{C} 2)\end{array}$ & \\
\hline DG 1 & 0.00 & 15.37 & 13.35 & 28.72 & \\
\hline DG 2 & 0.00 & 15.37 & 13.35 & 28.72 & \\
\hline Total & 0.00 & 30.74 & 26.70 & 57.45 & 57.45 \\
\hline
\end{tabular}

Table 12

The fuel consumption obtained from the simulation model.

\begin{tabular}{|c|c|c|c|c|c|c|c|}
\hline & \multicolumn{3}{|c|}{ Fuel consumption at berth (L) } & \multicolumn{3}{|c|}{ Fuel consumption while cruising (L) } & \multirow[t]{2}{*}{ Total fuel consumption for complete round trip (L) } \\
\hline & At terminal 1 & At terminal 2 & At berth $(\mathrm{T} 1+\mathrm{T} 2)$ & While cruising 1 & While cruising 2 & While cruising $(\mathrm{C} 1+\mathrm{C} 2)$ & \\
\hline DG 1 & 1.45 & 1.52 & 2.97 & 15.69 & 14.09 & 29.77 & \\
\hline DG 2 & 2.18 & 3.28 & 5.46 & 12.14 & 8.83 & 20.97 & \\
\hline Total & 3.62 & 4.80 & 8.43 & 27.83 & 22.92 & 50.75 & 59.17 \\
\hline
\end{tabular}


Table 15

Emissions obtained from HPS-RB.

\begin{tabular}{llll}
\hline & $\begin{array}{l}\mathrm{CO}_{2} \text { emissions, } \mathrm{E}_{\mathrm{CO} 2} \\
(\mathrm{~g} / \text { trip })\end{array}$ & $\begin{array}{l}\mathrm{SO}_{\mathrm{X}} \text { emissions, } \mathrm{E}_{\mathrm{SOx}} \\
(\mathrm{g} / \text { trip })\end{array}$ & $\begin{array}{l}\mathrm{NO}_{\mathrm{X}} \text { emissions, } \mathrm{E}_{\mathrm{NOx}} \\
(\mathrm{g} / \text { trip })\end{array}$ \\
\hline Berth & 0.00 & 0.00 & 0.00 \\
Cruising & 130614.67 & 2212.02 & 3160.03 \\
Total & 130614.67 & 2212.02 & 3160.03 \\
\hline
\end{tabular}

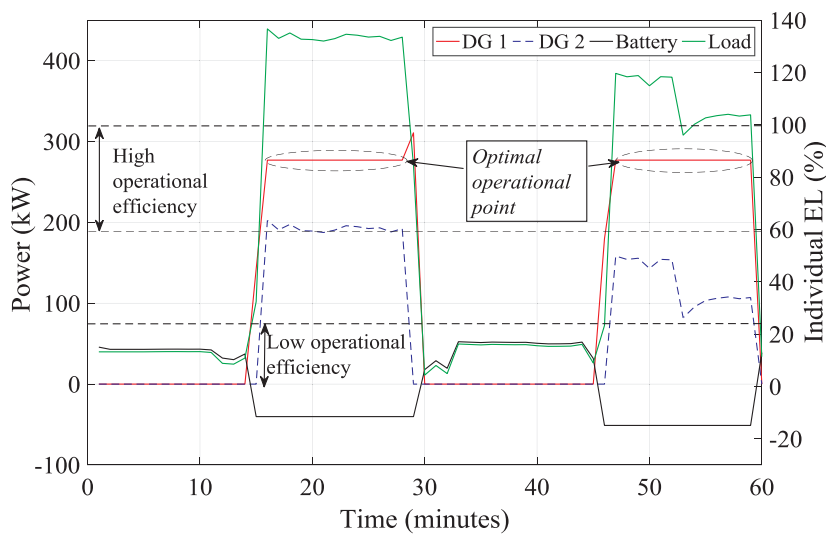

Fig. 22. The simulation output power of the proposed HPS-GWO.

close to a residential area. As both DGs are disconnected at this period, the $F C_{\text {berth }}$ is equal to zero.

At the first cruising period from 15 to $29 \mathrm{~min}$, DG 1 is operated at its optimal operational point which is $86.5 \%$ of engine load $(277 \mathrm{~kW})$. This results in a minimal SFOC of $0.266 \mathrm{~L} / \mathrm{kWh}$. In addition, DG 2 is maintained at approximately $58 \%-63 \%$ engine load by uniformly charging the battery over the complete cruising period. As a result, the fuel consumption of the first cruising period $F C_{\text {cruising1 }}$ is $29.70 \mathrm{~L}$. At the second cruising period from 46 to $59 \mathrm{~min}$, DG1 is also operated at its optimal operational point while DG2 is varying above the low operational efficiency range. This results in a fuel consumption of $25.05 \mathrm{~L}$ in the second cruising period. Therefore, the total fuel consumption of both DGs while cruising $\left(F C_{\text {cruising }}\right)$ is $54.75 \mathrm{~L}$. Overall, the total $F C_{\text {total }}$ for one round trip $(60 \mathrm{~min})$ of the HPS with a GWO based power management strategy is $54.75 \mathrm{~L}$. Table 16 shows the fuel consumption obtained by from HPS-GWO.

The total energy consumption is found to be $201.142 \mathrm{kWh}$, which is mainly occurred while cruising. The $\mathrm{CO}_{2}, \mathrm{SO}_{\mathrm{X}}$ and $\mathrm{NO}_{\mathrm{X}}$ emissions are calculated for both DGs and depicted in Table 17.

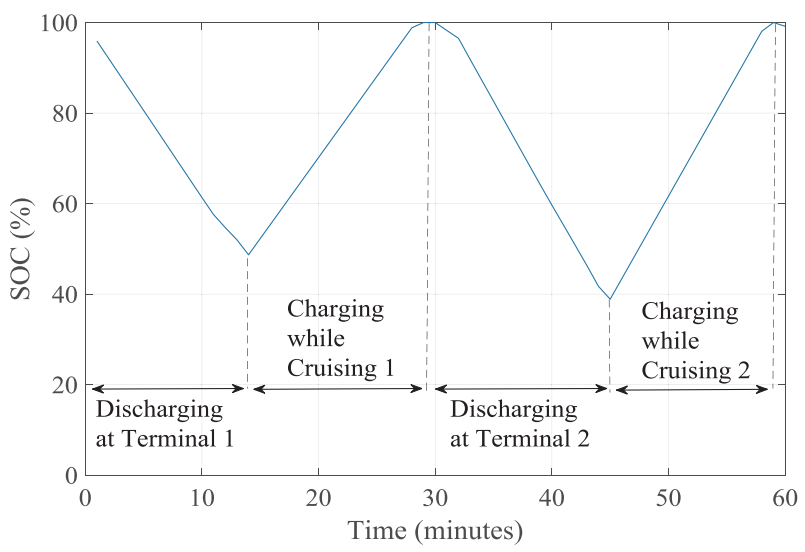

Fig. 23. The SOC of the BESS with HPS-GWO.
Table 16

Fuel consumption obtained from HPS-GWO.

\begin{tabular}{llllll}
\hline & $\begin{array}{l}\text { Fuel } \\
\text { consumption } \\
\text { at berth (L) }\end{array}$ & \begin{tabular}{l} 
Fuel consumption while cruising (L) \\
\cline { 3 - 5 }
\end{tabular} & $\begin{array}{l}\text { While } \\
\text { cruising 1 }\end{array}$ & $\begin{array}{l}\text { While } \\
\text { cruising } 2\end{array}$ & $\begin{array}{l}\text { While } \\
\text { cruising } \\
\text { consumption for } \\
\text { complete round } \\
\text { trip (L) }\end{array}$ \\
\hline DG 1 & 0.00 & 18.09 & 16.84 & 34.93 & \\
DG 2 & 0.00 & 11.61 & 8.21 & 19.82 & \\
Total & 0.00 & 29.70 & 25.05 & 54.75 & 54.75 \\
\hline
\end{tabular}

Table 17

Emissions obtained from HPS-GWO.

\begin{tabular}{llll}
\hline & $\begin{array}{l}\mathrm{CO}_{2} \text { emissions, } \mathrm{E}_{\mathrm{CO} 2} \\
(\mathrm{~g} / \text { trip })\end{array}$ & $\begin{array}{l}\mathrm{SO}_{\mathrm{x}} \text { emissions, } \mathrm{E}_{\mathrm{SOx}} \\
(\mathrm{g} / \text { trip })\end{array}$ & $\begin{array}{l}\mathrm{NO}_{\mathrm{x}} \text { emissions, } \mathrm{E}_{\mathrm{NOx}} \\
(\mathrm{g} / \text { trip })\end{array}$ \\
\hline Berth & 0.00 & 0.00 & 0.00 \\
Cruising & 124707.73 & 2111.99 & 3017.12 \\
Total & 124707.73 & 2111.99 & 3017.12 \\
\hline
\end{tabular}

\subsection{Comparison}

The main optimization parameters are powers of DG1, DG2 and battery. These parameters are optimized in order to keep the SFOC of the engine at optimal operational point and above low operational efficiency region. This results in fuel consumption reduction. The effect of those parameters on the SFOC and hence the fuel consumption of engine are summarized below.

Effects of the DG powers (DG1 and DG2) on the SFOC: As per Eq. (2) and corresponding Fig. 17, the increase of the generated power from the DG reduces the SFOC value until a certain point (optimal point) then it starts to increase slightly with the increase of DG power. Fig. 17 depicts the effect of the variation of engine load (DG power) on the SFOC. As shown in this Fig. 17, the optimal SFOC point is $0.266 \mathrm{~L} / \mathrm{kWh}$ at $86.5 \%$ engine load $(277 \mathrm{~kW})$ which falls in the high operational efficiency region. In the high operational efficiency region, the maximum SFOC increment compared to the optimal point is $4.51 \%$. In the low operational efficiency region, the maximum and minimum SFOC are $0.391 \mathrm{~L} / \mathrm{kWh}$ and $0.330 \mathrm{~L} / \mathrm{kWh}$ at $1 \%$ and $25 \%$ engine load respectively $(3.2 \mathrm{~kW}$ and $80 \mathrm{~kW})$. In this region, the maximum and minimum SFOC increment compared to the optimal point are $46.99 \%$ and $24.04 \%$ respectively. Therefore, optimizing the power output of DG will results in more fuel consumption reduction.

Effects of battery power on SFOC: The battery is used to supply power when the ferry operated at low demand (at terminal). The use of the battery as a main power supply at terminal will reduce the total fuel consumption and eliminate emissions and noises as both DGs are not operating. At cruising periods, when the battery is charging, the power of the battery is optimized in the way that it gets charged in a uniform rate of charge. This will increase the load on the on-board DGs which will make the DGs operate at higher engine load over the complete cruising period. This will results in operating the engines above the low efficiency region over the entire cruising period which results in minimizing the SFOC and hence the fuel consumption.

The results of the SFOC and fuel consumption using RB method and GWO method compared to the existing AC system are shown in Fig. 24 and Table 18. In the existing AC system, both DGs are operated at all times. As it can be seen from Fig. 24, both DGs are operated at low engine load at terminal 1 and 2. This attribute to significant increase in the SFOC. The results show that the proposed HPS using RB and GWO method provide $100 \%$ reduction of SFOC at terminals (at berth) as both DGs are switched off and the BESS supplies power to the load. This results in a quiet operation (no noise from the DGs as both are switched off) and eliminates emissions at the terminal which is close to a 


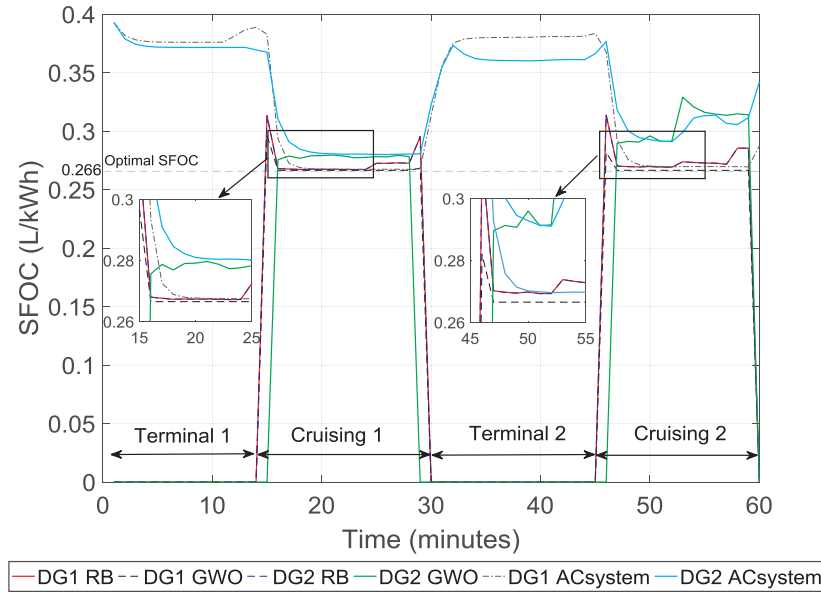

Fig. 24. SFOC results of the AC system and the proposed HPS using RB and GWO method.

residential area. In addition, as shown in Fig. 24, DG1 and DG2 produce same SFOC by using RB method, as both DGs are equally share the load power. In contrast, by using GWO method, DG1 always operates at optimal SFOC while DG2 is dealing with the load variation above the low operational efficiency range. Moreover, the operating hours of DG2 is reduced by three minutes compared to RB method.

The fuel consumptions in liters of each system are summarized in Table 18. With the HPS-RB, the fuel consumption of the DGs is reduced by $2.91 \%$ despite that the energy consumption of the DGs being increased in order to charge the BESS. This is due to the fact that both DGs are not operated at their optimal operational efficiency level. With the HPS-GWO, more fuel consumption reduction is achieved with a percentage of $7.48 \%$ and additionally the energy consumption of the DGs is reduced since DG 1 is always operated at its optimal point and
DG 2 is always operated near its high operational range. Table 18 shows the summary of fuel consumption for each system.

According to Table 18, there is a slight increment of fuel consumption in the proposed systems during cruising compared to the existing AC system. This is due to the extra power generated by the engines to charge the BESS. The HPS-GWO provided lower fuel consumption in the cruising period compared to the HPS-RB. This is due to the operation of DG 1 at its optimal operational point and eliminating the operation of DG 2 in the low operational range. This is done by uniformly charging the battery while cruising. On the other hand, as RB method uses a pre-determined condition to control the system, the HPS$\mathrm{RB}$ runs both DGs at the same engine load by equally dividing the load power between both DGs. Overall, the HPS-RB and HPS-GWO provided $57.45 \mathrm{~L}$ and $54.75 \mathrm{~L}$ of fuel consumption respectively. The fuel consumption of each power system at different ferry operating conditions is shown in Fig. 25.

The results show that the HPS provides a zero-emissions solution at berth. The energy consumption for each operating condition is used to calculate the emissions. The total energy consumption for the existing AC system, HPS-RB and HPS-GWO is $202.9 \mathrm{kWh}, 210.67 \mathrm{kWh}$ and $201.14 \mathrm{kWh}$ respectively as shown in Fig. 26.

The proposed HPS with BESS provided a $100 \%$ emissions reduction at terminal using both power management methods as shown in Table 19. Therefore, such HPS with BESS provide an efficient solution to eliminate berth emission for short-haul ferries that operate close to urban areas where the reduction of emissions is required to reduce the direct impact of emissions on human health. However, in the cruising period, the HPS-RB provide a $15.79 \%$ and HPS-GWO provide $10.56 \%$ increment in $\mathrm{CO}_{2}, \mathrm{SO}_{\mathrm{X}}$ and $\mathrm{NO}_{\mathrm{x}}$. This is due to the increase of the DGs' energy production to charge the on-board battery while cruising. In the HPS-RB, the $\mathrm{CO}_{2}, \mathrm{SO}_{\mathrm{X}}$ and $\mathrm{NO}_{\mathrm{X}}$ emissions increase by $2.76 \%, 2.71 \%$ and $6.01 \%$ respectively. In contract, in the HPS-GWO, $\mathrm{CO}_{2}$ and $\mathrm{SO}_{\mathrm{X}}$ emissions are reduced by $1.88 \%$ and $1.93 \%$ respectively with slight increment of $\mathrm{NO}_{\mathrm{X}}$ emission by $1.22 \%$.

Table 18

Fuel consumption comparison among the three systems for one round trip.

\begin{tabular}{|c|c|c|c|c|c|c|c|}
\hline & $\begin{array}{l}\text { Fuel consumption at } \\
\text { berth (L) }\end{array}$ & $\begin{array}{l}\text { Fuel consumption while } \\
\text { cruising (L) }\end{array}$ & $\begin{array}{l}\text { Total fuel consumption for } \\
\text { one round trip (L) }\end{array}$ & $\begin{array}{l}\text { Fuel consumption } \\
\text { reduction }(\mathrm{L})\end{array}$ & $\begin{array}{l}\text { Fuel costs } \\
\text { (AUD\$) }\end{array}$ & $\begin{array}{l}\text { Fuel savings } \\
\text { (AUD\$) }\end{array}$ & $\begin{array}{l}\text { Fuel consumption } \\
\text { reduction }(\%)\end{array}$ \\
\hline $\begin{array}{c}\text { Existing AC } \\
\text { system }\end{array}$ & 8.43 & 50.75 & 59.17 & - & 83.133 & - & - \\
\hline HPS-RB & 0.00 & 57.45 & 57.45 & 1.72 & 80.717 & 2.146 & 2.91 \\
\hline HPS-GWO & 0.00 & 54.75 & 54.75 & 4.43 & 76.923 & 6.21 & 7.48 \\
\hline
\end{tabular}

a Terminal $1 \square$ Terminal $2 \square$ Cruising $1 \backsim$ Cruising $2 \square$ Total

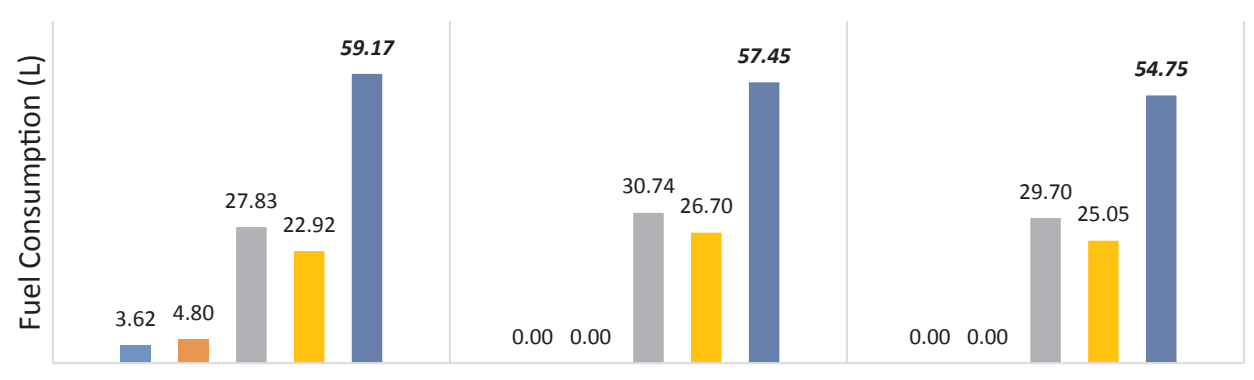

AC system

HPS-RB

HPS-GWO

Fig. 25. Fuel consumption comparison of each power system. 

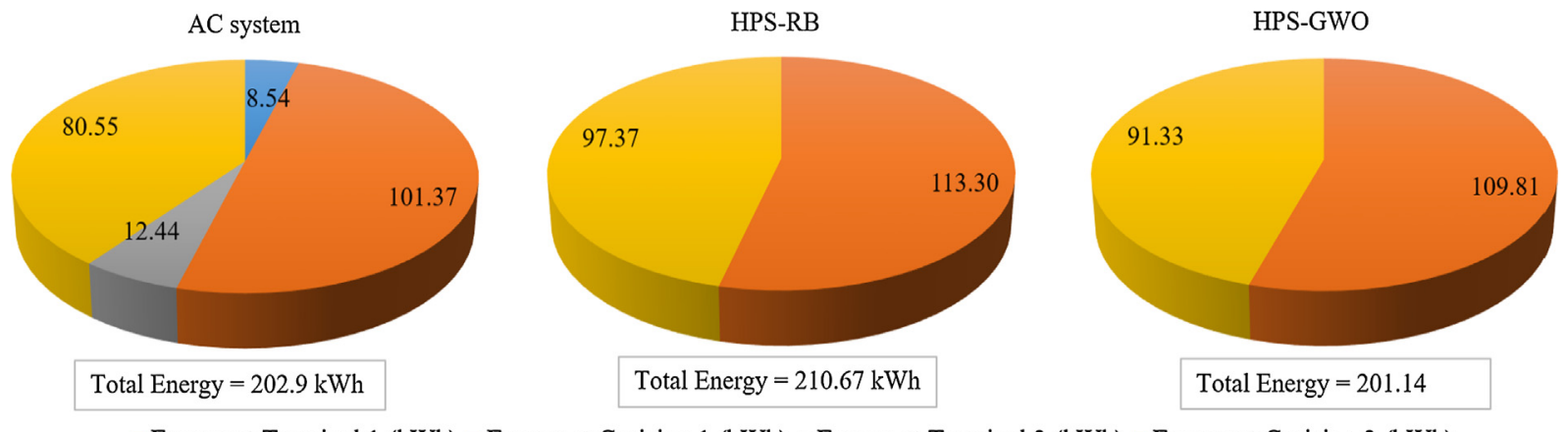

$\varpi$ Energy at Terminal $1(\mathrm{kWh}) \bowtie$ Energy at Cruising $1(\mathrm{kWh}) \bowtie$ Energy at Terminal $2(\mathrm{kWh}) \llbracket$ Energy at Cruising $2(\mathrm{kWh})$

Fig. 26. Energy consumption of DGs for each system at different operating conditions.

Table 19

The emissions produced from each system for different operating conditions.

\begin{tabular}{|c|c|c|c|c|c|c|c|c|c|}
\hline & \multicolumn{3}{|c|}{ Emissions at berth (g/trip) } & \multicolumn{3}{|c|}{ Emissions while cruising (g/trip) } & \multicolumn{3}{|c|}{ Emissions for a complete voyage (g/trip) } \\
\hline & $\mathrm{CO}_{2}$ & $\mathrm{SO}_{\mathrm{X}}$ & $\mathrm{NO}_{\mathrm{X}}$ & $\mathrm{CO}_{2}$ & $\mathrm{SO}_{\mathrm{X}}$ & $\mathrm{NO}_{\mathrm{X}}$ & $\mathrm{CO}_{2}$ & $\mathrm{SO}_{\mathrm{X}}$ & $\mathrm{NO}_{\mathrm{X}}$ \\
\hline AC system & 14306.27 & 243.33 & 251.72 & 112794.20 & 1910.22 & 2728.89 & 127100.47 & 2153.56 & 2980.62 \\
\hline HPS-RB & 0.00 & 0.00 & 0.00 & 130614.67 & 2212.02 & 3160.03 & 130614.67 & 2212.02 & 3160.03 \\
\hline HPS-GWO & 0.00 & 0.00 & 0.00 & 124707.73 & 2111.99 & 3017.12 & 124707.73 & 2111.99 & 3017.12 \\
\hline Reduction using HPS-RB & $100 \%$ & $100 \%$ & $100 \%$ & $-15.79 \%$ & $-15.79 \%$ & $-15.79 \%$ & $-2.76 \%$ & $-2.71 \%$ & $-6.01 \%$ \\
\hline Reduction using HPS-GWO & $100 \%$ & $100 \%$ & $100 \%$ & $-10.56 \%$ & $-10.56 \%$ & $-10.56 \%$ & $1.88 \%$ & $1.93 \%$ & $-1.22 \%$ \\
\hline
\end{tabular}

\section{Conclusions}

The DC-HPS with BESS can be considered as a promising solution to reduce the fuel consumption and thereby reduce the emissions in ferries. In order to optimally share the power among all HPS components in such complex systems, an efficient power management strategy (PMS) is essential. This paper highlights the advantage of using a HPS with DC distribution and BESS over an AC power system for a shorthaul RORO ferry. Two PMSs, namely RB and GWO, are proposed and implemented on the proposed HPS system. Performance comparisons of the two PMSs are carried out based on two evaluating indicators: fuel consumption and emissions reductions. The MATLAB/Simulink software is used to model and simulate the system. A case study with measured load profile of a short-haul RORO ferry is used to validate and examine the proposed system.

Simulation results showed that both methods, the HPS-RB and HPSGWO, provide a $100 \%$ reduction in fuel consumption at berth and a $2.91 \%$ and $7.48 \%$ reduction respectively during the complete voyage. A greater reduction is achieved with HPS-GWO as it operates the DG 1 at its optimal operational point and avoids running the DG 2 in the low operational range. This is achieved by uniformly charging the BESS while cruising, in order to maintain the engine performance at highest engine load over the entire cruising period.

In terms of emissions, the simulation results showed that the HPS$\mathrm{RB}$ and HPS-GWO offer $100 \%$ emissions reductions at berth. In the cruising period, the HPS-RB provide a $15.79 \%$ and HPS-GWO provide $10.56 \%$ increment in $\mathrm{CO}_{2}, \mathrm{SO}_{\mathrm{X}}$ and $\mathrm{NO}_{\mathrm{X}}$. This is due to an increase in the DGs' energy production to charge the on-board battery during cruising. However, the HPS-GWO provide $1.88 \%$ and $1.93 \%$ reduction of $\mathrm{CO}_{2}$ and $\mathrm{SO}_{\mathrm{X}}$ emissions respectively with $1.22 \%$ increment of $\mathrm{NO}_{\mathrm{X}}$ in the complete voyage.

Overall, the BESS integration into a short-haul ferry power system can be considered as an effective solution to reduce emissions and noise at the berth. This provides fuel consumption reductions as diesel engines' operating hours are reduced since they are shut down at the berth. In terms of power system architecture, a HPS with DC distribution is considered an effective architecture when incorporating an ESS into a ferry power system. This is due to system flexibility in operating each diesel engine at different speeds. For the PMSs, a meta-heuristic on-line optimization method, GWO, provides better fuel consumption and emissions reductions compared to a classical RB method which uses pre-determined conditions. This is due to the capability of GWO in solving multi objective optimization problems with several operational constraints to find the optimal solution in contrast to classical methods that use pre-determined conditions. Taken together, these results suggest that the DC - HPS with the BESS and the GWO-based power management strategy is a good combination to achieve low fuel consumption, low emissions and eliminate noises at berth for short-haul hybrid electric ferries. The insight gained from this study can be of assistance to marine coastal vehicles operating in close proximity to residential areas where both noise and air emissions are of concern.

Future work is required to develop a hybrid optimization method by combining two or more optimization techniques for more accurate and promising optimization results. In addition, studies could be extended to compare the performance of GWO with other meta-heuristic optimization for power management optimization of electric ferries. Moreover, recommendations for repetitive shutdown-restart of the engines should also be considered in the overall assessment of the proposed system. Research incorporating local controller with each component could also be conducted to improve the operation of the optimizer.

\section{References}

[1] Livanos GA, Theotokatos G, Pagonis D-N. Techno-economic investigation of alternative propulsion plants for Ferries and RoRo ships. Energy Convers Manage 2014;79:640-51.

[2] Nielsen RF, Haglind F, Larsen U. Design and modeling of an advanced marine machinery system including waste heat recovery and removal of sulphur oxides. Energy Convers Manage 2014;85:687-93.

[3] Shancita I, Masjuki HH, Kalam MA, Rizwanul Fattah IM, Rashed MM, Rashedul HK. A review on idling reduction strategies to improve fuel economy and reduce exhaust emissions of transport vehicles. Energy Convers Manage 2014;88:794-807.

[4] Dimopoulos GG, Georgopoulou CA, Stefanatos IC, Zymaris AS, Kakalis NMP. A general-purpose process modelling framework for marine energy systems. Energy Convers Manage 2014:86:325-39.

[5] García Valladolid P, Tunestål P, Monsalve-Serrano J, García A, Hyvönen J. Impact of diesel pilot distribution on the ignition process of a dual fuel medium speed marine engine. Energy Convers Manage 2017;149:192-205.

[6] Hansen JF, Wendt F. Historyand state of the art in commercial electric ship 
propulsion, integrated power systems, and future trends. Proc IEEE 2015;103:2229-42.

[7] Dedes EK, Hudson DA, Turnock SR. Assessing the potential of hybrid energy technology to reduce exhaust emissions from global shipping. Energy Policy 2012;40:204-18.

[8] García-Olivares A, Solé J, Osychenko O. Transportation in a 100\% renewable energy system. Energy Convers Manage 2018;158:266-85.

[9] Ling-Chin J, Roskilly AP. Investigating a conventional and retrofit power plant onboard a Roll-on/Roll-off cargo ship from a sustainability perspective - A life cycle assessment case study. Energy Convers Manage 2016;117:305-18.

[10] Zahedi B, Norum LE, Ludvigsen KB. Optimized efficiency of all-electric ships by dc hybrid power systems. J Power Sources 2014;255:341-54.

[11] Staudt V, Bartelt R, Heising C. Fault scenarios in DC ship grids. IEEE Electrif Mag 2015;3:40-8.

[12] Castellan S, Menis R, Tessarolo A, Luise F, Mazzuca T. A review of power electronics equipment for all-electric ship MVDC power systems. Int J Electr Power Energy Syst 2018;96:306-23.

[13] Jin Z, Sulligoi G, Cuzner R, Meng L, Vasquez JC, Guerrero JM. Next-generation shipboard dc power system: Introduction smart grid and dc microgrid technologies into maritime electrical networks. IEEE Electrif Mag 2016;4:45-57.

[14] Kassakian JG, Jahns TM. Evolving and emerging applications of power electronics in systems. IEEE J Emerging Selected Topics Power Electron 2013;1:47-58.

[15] Zahedi B, Norum LE, Ludvigsen KB. Optimization of fuel consumption in shipboard power systems. In: Presented at the IECON 2013 - 39th annual conference of the IEEE industrial electronics society; 2014.

[16] Zahedi B, Norum LE. Modeling and simulation of all-electric ships with low-voltage DC hybrid power systems. IEEE Trans Power Electron 2013;28:4525-37.

[17] Skjong E, Volden R, Rodskar E, Molinas M, Johansen TA, Cunningham J. Past, present, and future challenges of the marine Vessel's electrical power system. IEEE Trans Transp Electrif 2016;2:522-37.

[18] Kanellos FD, Prousalidis J, Tsekouras GJ. Optimal active power management in all electric ship employing DC grid technology. Operational research in business and economics. Springer; 2017. p. 271-84.

[19] Ling-Chin J, Roskilly AP. A comparative life cycle assessment of marine power systems. Energy Convers Manage 2016;127:477-93.

[20] Baldi F, Ahlgren F, Melino F, Gabrielii C, Andersson K. Optimal load allocation of complex ship power plants. Energy Convers Manage 2016;124:344-56.

[21] Gagatsi E, Estrup T, Halatsis A. Exploring the potentials of electrical waterborne transport in Europe: The E-ferry concept. Transp Res Procedia 2016;14:1571-80.

[22] Klebanoff LE, Pratt JW, Leffers CM, Sonerholm KT, Escher T, Burgard J, et al. Comparison of the greenhouse gas and criteria pollutant emissions from the SFBREEZE high-speed fuel-cell ferry with a diesel ferry. Transp Res Part D: Transport Environ 2017;54:250-68.

[23] Merien-Paul RH, Enshaei H, Jayasinghe SG. In-situ data vs. bottom-up approaches in estimations of marine fuel consumptions and emissions. Transp Res Part D: Transport Environ 2018;62:619-32.

[24] McArthur DP, Osland L. Ships in a city harbour: An economic valuation of atmospheric emissions. Transp Res Part D: Transport Environ 2013;21:47-52.

[25] Winnes H, Styhre L, Fridell E. Reducing GHG emissions from ships in port areas. Res Transp Business Manage 2015;17:73-82.

[26] Merk O. Shipping emissions in ports. Presented at the international transport forum; 2014.

[27] Tichavska M, Tovar B. Port-city exhaust emission model: An application to cruise and ferry operations in Las Palmas Port. Transp Res Part A: Policy Practice 2015;78:347-60.

[28] Sciberras EA, Zahawi B, Atkinson DJ, Juando A, Sarasquete A. Cold ironing and onshore generation for airborne emission reductions in ports. Proc Inst Mech Eng, Part M: J Eng Maritime Environ 2014;230:67-82.

[29] Jayasinghe SG, Al-Falahi M, Enshaei H, Fernando N, Tashakori A. Floating power platforms for mobile cold-ironing. In: Presented at the 2016 IEEE 2nd annual southern power electronics conference (SPEC), Auckland; 2016.

[30] Sciberras EA, Zahawi B, Atkinson DJ. Reducing shipboard emissions - Assessment of the role of electrical technologies. Transp Res Part D: Transport Environ 2017;51:227-39.

[31] Misyris GS, Marinopoulos A, Doukas DI, Tengnér T, Labridis DP. On battery state estimation algorithms for electric ship applications. Electr Power Syst Res 2017;151:115-24.

[32] Zanne M, Počuča M, Bajec P. Environmental and economic benefits of slow steaming. Trans Maritime Sci 2013;02:123-7.

[33] Skjong E, Rødskar E, Molinas M, Johansen TA, Cunningham J. The marine vessel's electrical power system: From its birth to present day. Proc IEEE 2015;103(12):2410-24.

[34] Al-falahi MDA, Jayasinghe SDG, Enshaei H. A review on recent size optimization methodologies for standalone solar and wind hybrid renewable energy system.
Energy Convers Manage 2017;143:252-74.

[35] Chauhan A, Saini RP. A review on integrated renewable energy system based power generation for stand-alone applications: Configurations, storage options, sizing methodologies and control. Renew Sustain Energy Rev 2014;38:99-120.

[36] Bassam AM, Phillips AB, Turnock SR, Wilson PA. Design, modelling and simulation of a hybrid fuel cell propulsion system for a domestic ferry. In: PRADS2016, Copenhagen, Denmark; 2016.

[37] Choi CH, Yu S, Han I-S, Kho B-K, Kang D-G, Lee HY, et al. Development and demonstration of PEM fuel-cell-battery hybrid system for propulsion of tourist boat. Int J Hydrogen Energy 2016;41:3591-9.

[38] Bianucci M, Merlino S, Ferrando M, Baruzzo L. The optimal hybrid/electric ferry for the Liguria Natural Parks. In: Presented at the OCEANS 2015 - Genova, Genova, Italy; 2015.

[39] Han J, Charpentier J-F, Tang T. An energy management system of a fuel cell/battery hybrid boat. Energies 2014;7:2799-820.

[40] Bassam AM, Phillips AB, Turnock SR, Wilson PA. An improved energy management strategy for a hybrid fuel cell/battery passenger vessel. Int J Hydrogen Energy 2016;41:22453-64.

[41] Trieste S, Hmam S, Olivier JC, Bourguet S, Loron L. Techno-economic optimization of a supercapacitor-based energy storage unit chain: Application on the first quick charge plug-in ferry. Appl Energy 2015;153:3-14.

[42] Jin Z, Meng L, Guerrero JM, Han R. Hierarchical control design for a shipboard power system with DC distribution and energy storage aboard future more-electric ships. IEEE Trans Ind Inf 2018;14:703-14.

[43] Jalkanen JP, Johansson L, Kukkonen J, Brink A, Kalli J, Stipa T. Extension of an assessment model of ship traffic exhaust emissions for particulate matter and carbon monoxide. Atmos Chem Phys 2012;12:2641-59.

[44] Adnanes AK. Maritime electrical installations and diesel electricpropulsion. ABB AS Marine 2003.

[45] Ghobadian B, Rahimi H, Nikbakht AM, Najafi G, Yusaf TF. Diesel engine performance and exhaust emission analysis using waste cooking biodiesel fuel with an artificial neural network. Renew Energy 2009;34:976-82.

[46] Du Y, Chen Q, Quan X, Long L, Fung RYK. Berth allocation considering fuel consumption and vessel emissions. Transp Res Part E: Logistics Transp Rey 2011;47:1021-37.

[47] Goldsworthy L, Goldsworthy B. Modelling of ship engine exhaust emissions in ports and extensive coastal waters based on terrestrial AIS data - An Australian case study. Environ Modell Software 2015;63:45-60.

[48] Cummins engine DS72-CPGK. C. P. Generation, Ed., ed; 2012.

[49] Jahangiri S, Kam US, Garaniya V, Abbassi R, Enshaei H, Brown RJ, et al. Development of emission factors for ships' emissions at berth. In: Presented at the coasts \& ports 2017 conference - cairns, Queensland, Australia; 2017.

[50] Baird AJ, Pedersen RN. Analysis of $\mathrm{CO} 2$ emissions for island ferry services. J Transp Geogr 2013;32:77-85.

[51] Song S. Ship emissions inventory, social cost and eco-efficiency in Shanghai Yangshan port. Atmos Environ 2014;82:288-97.

[52] Entec. Defra UK ship emissions inventory, https://uk-air.defra.gov.uk/assets/ documents/reports/cat15/1012131459 21897 Final Report 291110.pdf2010.

[53] Stavrakaki A, Jonge ED, Hugi C, Whall C, Minchin W, Ritchie A, et al. Service contract on ship emissions: assignment, abatement and market-based instruments. Entec UK Limited August 2005; 2005.

[54] Yin C, Wu H, Locment F, Sechilariu M. Energy management of DC microgrid based on photovoltaic combined with diesel generator and supercapacitor. Energy Convers Manage 2017;132:14-27.

[55] Tie SF, Tan CW. A review of energy sources and energy management system in electric vehicles. Renew Sustain Energy Rev 2013;20:82-102.

[56] Mirjalili S, Mirjalili SM, Lewis A. Grey wolf optimizer. Adv Eng Softw 2014;69:46-61.

[57] Sulaiman MH, Mustaffa Z, Mohamed MR, Aliman O. Using the gray wolf optimizer for solving optimal reactive power dispatch problem. Appl Soft Comput 2015;32:286-92.

[58] Nimma KS, Al-Falahi MD, Nguyen HD, Jayasinghe S, Mahmoud TS, Negnevitsky M. Grey wolf optimization-based optimum energy-management and battery-sizing method for grid-connected microgrids. Energies 2018;11:847.

[59] Mirjalili S. How effective is the Grey Wolf optimizer in training multi-layer perceptrons. Appl Intelligence 2015;43:150-61.

[60] Al-Falahi M, Tarasiuk T, Jayasinghe S, Jin Z, Enshaei H, Guerrero J. AC ship mi crogrids: control and power management optimization. Energies 2018;11:1458.

[61] PBES. 2017, August 2017. http://www.pbes.com/wp-content/uploads/2017/06/ PBES_Power-Energy_2017-06-16.pdf.

[62] CORVUS. August 2017. http://corvusenergy.com/wp-content/uploads/2015/09/ Corvus-Energy-Overview Feb2016.pdf.

[63] Rashid MH. Pulse-width-modulation inverters. Prentice-Hall; 2004. p. 237-48. 


\section{Chapter 5: \\ Performance Improvements of Using Hybrid Meta-Heuristic- Based Optimization Compared to Single Meta-Heuristic-Based Optimization}

This chapter presents design and development of a novel hybrid meta-heuristic algorithm-based PMS for the fuel savings of hybrid electric ferries and compares its performance with that of single meta-heuristic algorithm. The proposed hybrid PMS method applies an interactive approach based on a GWO and fuzzy expert system to improve the computational efficiency of the algorithm. The performance of the proposed FL-GWO algorithm is compared with that of GWO algorithm and assessed in terms of fuel consumption, operating cost, and standard deviation. Two load scenarios are simulated: normal and high loads. This chapter has been published in The International Journal of Energy (Elsevier). The citation of the article is:

M. D. Al-Falahi, S. Jayasinghe, and H. Enshaei, "Hybrid Algorithm for Optimal Operation of Hybrid Power Systems in Electric Ferries,” Energy, vol.187, p115923. 


\title{
Hybrid algorithm for optimal operation of hybrid energy systems in electric ferries
}

\author{
Monaaf D.A. Al-Falahi*, Shantha D.G. Jayasinghe, Hossein Enshaei \\ Australian Maritime College, University of Tasmania, Newnham, TAS, 7248, Australia
}

\section{A R T I C L E I N F O}

\section{Article history:}

Received 29 April 2019

Received in revised form

24 June 2019

Accepted 9 August 2019

Available online 13 August 2019

\section{Keywords:}

Battery energy storage system

Electric ferry

Hybrid algorithm

Hybrid energy system

Power management system

\begin{abstract}
A B S T R A C T
The move towards electrification of marine vessels enables the development of more efficient vessels by reducing fuel consumption and emissions. This includes incorporating electrical energy sources, storage systems and interfacing power electronic converters which increase system complexity. Therefore, an accurate and efficient power management system (PMS) is essential to achieve the optimum operation. This study aims to develop a novel hybrid meta-heuristic algorithm-based PMS for the fuel savings of hybrid electric ferries. The ferry power system used in this study comprises two diesel generator sets and a battery storage system. The proposed hybrid PMS method applies an interactive approach on the basis of a grey wolf optimizer (GWO) and fuzzy expert system to improve the computational efficiency of the algorithm. Measured load data from an existing short-haul ferry are used in the simulation under two load scenarios: normal and high load demands. The proposed fuzzy logic-grey wolf optimizer (FL-GWO) aims to minimize the operating cost of the proposed system while satisfying all operational and technical constraints of the ferry. Results show that the proposed FL-GWO provided a more accurate optimal solution set with less standard deviation than the GWO. The proposed method realized up to $3.14 \%$ and $1.81 \%$ fuel savings in normal- and high-load scenarios, respectively, compared with GWO. Moreover, the sensitivity analysis indicates that charging the battery from the onboard generators in a more uniform rate over the entire cruising period reduces the fuel consumption.
\end{abstract}

๑) 2019 Elsevier Ltd. All rights reserved.

\section{Introduction}

The electrification of marine vessels by integrating more electric technologies is a promising solution for fuel efficiency improvement and emission reduction [1]. Although electric propulsion has these advantages, electrical power still comes from prime-moverdriven generators. Therefore, the industry and academia are actively exploring various technologies that can help reduce emissions and increase energy savings [2] ranging from the use of low-emission fuels such as liquefied natural gas to further electrification, by increasing the hybridization $[1,3]$. This case has opened up the integration of renewable energy sources (RESs) and energy storage systems into ship power system [4,5]. However, typical power levels and densities of RESs are still not high enough to meet the requirements of large ships. In addition, large energy storage systems are required to satisfy their loads; thus, technical and

\footnotetext{
* Corresponding author.

E-mail addresses: monaaf.alfalahi@utas.edu.au (M.D.A. Al-Falahi), shanthaj@ utas.edu.au (S.D.G. Jayasinghe), hossein.enshaei@utas.edu.au (H. Enshaei).
}

economic factors need to be taken into account to justify the hybridization for large ships with RESs and/or energy storage systems $[6,7]$.

Nevertheless, short-haul ferries, which operate for a short distance with a relatively low power demand, are suitable for RESs and energy storage systems integration. However, the slow dynamics or intermittent nature of RESs prevents them from being the main power source [1]. Consequently, battery energy storage system has become an integral part for ensuring a reliable power supply. Therefore, the hybridization of ferries by integrating a battery energy storage system has gained considerable attention in recent years $[6,8,9]$.

\subsection{Trends of battery integration into ferries}

The trend towards integration of batteries into ferries has gained close attention in recent years. MV Halaing, the first hybrid electric ferry with battery storage, started its operation in 2013 and recorded significant fuel savings and emission reductions [10]. Following the same trend, Ampere ferry in Norway, which is the first battery- 


\begin{tabular}{|c|c|c|c|}
\hline \multicolumn{2}{|c|}{ Nomenclature } & \multirow{2}{*}{$\begin{array}{l}g \\
\eta\end{array}$} & \multirow{2}{*}{$\begin{array}{l}\text { DG operating variable [0 or } 1 \text { ] } \\
\text { Efficiency }\end{array}$} \\
\hline$E_{B}$ & Battery energy [kWh] & & \\
\hline$P_{B}$ & Battery power $[\mathrm{kW}]$ & \multicolumn{2}{|c|}{ Abbreviation } \\
\hline$P_{L}$ & Load power $[\mathrm{kW}]$ & GWO & Grey wolf optimization \\
\hline$P_{c h a}$ & Charging power $[\mathrm{kW}]$ & HPS & Hybrid power system \\
\hline$P_{d c h a}$ & Discharging power [kW] & IMO & International maritime organization \\
\hline$P_{n}$ & Generated power from n-th generator [kW] & $\mathrm{NO}_{\mathrm{X}}$ & Nitrogen oxide \\
\hline$P_{n}^{\max }$ & Maximum power of n-th generator [kW] & PMS & Power management system \\
\hline$P_{t n}$ & Power generated by $n$-th generator at t-th time $[\mathrm{kW}]$ & RES & Renewable energy source \\
\hline SFOC & Specific fuel oil consumption [L/kWh] & SD & Standard deviation \\
\hline $\mathrm{OC}$ & Operating costs [AUD\$] & $\mathrm{F}$ & Fuel consumption (L) \\
\hline SOChigh & Safe value of SoC limit [\%] & & \\
\hline SOC ${ }^{\max }$ & Maximum SoC [\%] & \multicolumn{2}{|l|}{ Subscripts } \\
\hline $\mathrm{SOC}^{\mathrm{min}}$ & Minimum state of charge [\%] & $t_{c}$ & The index time of charging \\
\hline$\Delta_{t}$ & Time step & $B$ & Battery \\
\hline DoD & Depth of discharge [\%] & cha & Charging \\
\hline E & Energy [kWh] & discha & Discharging \\
\hline$E L$ & Engine load & $E L$ & Engine load \\
\hline$N$ & Number of DGs & $L$ & Load \\
\hline$T$ & The total time period & $\max$ & Maximum \\
\hline$b$ & BESS operating variable ['0' discharge, ' 1 ' charge] & $\min$ & Minimum \\
\hline
\end{tabular}

powered ferry in the world, started its operation in 2015 and reported 60\% savings of fuel in 2016 and zero emissions [10]. Another successful example is Finland's first hybrid ferry, Elektra, which started operating in 2017 using batteries as main power supply with diesel generators, reduced $60 \%$ of $\mathrm{CO}_{2}$ emissions with low electricity costs per crossing, and provided large operating cost savings [11]. These successful implementations have attracted many ferry operators and owners to invest in hybrid and batterypowered ferries especially in Europe due to introduction of emissions control areas (ECAs) in the European waters [12]. The investment in such clean ferries is not only on building new ferries with battery storage systems but also on retrofitting some of the current electric ferries by integrating a battery system. Such battery integration into existing diesel-electric ferries can provide up to $65 \%$ of emission reduction with high operating cost savings. This scheme was founded by a major project co-financed by European Union in which several diesel-electric ferries was retrofitted to hybrid battery-diesel electric ferries, such as M/F Yycho Brahe in Demark [13].

The trend continues with an increasing number of ferries are being built with hybrid and fully battery-powered system due to their advantages of fuel savings and emission and noise reductions, especially as most ferries operate close to human habitation areas where emissions and noises are of concerns $[10,14]$. Many batterypowered and hybrid ferries and boats that move through inland waterways in the Netherlands, Norway, Belgium, and Canada are being built and soon to make their first voyage [15-17]. Only Norway will renew and build over 20 ferries with a battery storage system in the next few years [12].

The challenge in such hybrid system is matching the characteristic of the battery storage system (discharge and charge rates) and other generation units to meet the load requirements. The way that the system is operated can significantly affect the operating costs and the battery lifetime. Moreover, the efficient operation of the system will maximize the benefits of using such hybrid- and allelectric systems with batteries. In this context, the development of efficient power management strategies along with the progressive development in batteries will help further increase in the trend of battery-powered and hybrid electric ferries with battery storage systems.

\subsection{Power management optimization for hybrid electric ferries}

In a hybrid system, the presence of multi-generation sources and energy storage systems increases the complexity of the ferry power system [18]. Consequently, an efficient approach of operating such a system is required while satisfying ferry operational constraints $[8,19]$. Therefore, the deployment of a well-designed and advanced power management system (PMS) for short-haul ferries with a hybrid power system will provide an efficient system operation, thereby minimizing fuel consumption. The main targets of the PMS are cost minimization and emission limitation, which can be achieved through fuel consumption minimization. These targets combined with the compliance of ferry operational constraints render the optimal power management a complex problem.

Out of the PMSs found in the literature, the multi-scheme, which uses a deterministic rule-based method with preplanned states is the simplest to manage the power flow. This PMS has been applied for hybrid ferries and boats in Refs. [9,20-24]. Despite its simplicity, several innumerable contingencies that may present during vessel operation and thus preplanned power management can result in suboptimal fuel efficiency $[25,26]$. Therefore, further advanced PMSs that can ensure the global optimum operation under any given condition are vital.

The advanced PMS-based meta-heuristic optimization algorithm is a promising approach for matching the power generation and the load demand, while meeting the operational constrains to reduce fuel consumption and emissions [27-29]. Furthermore, the use of a hybrid meta-heuristic algorithm (by combining two or more algorithms) can provide more promising optimization results for hybrid energy systems than the single meta-heuristic algorithm [28,30-34]. Nevertheless, few studies have applied the metaheuristic algorithm in solving several optimization problems for shipboard power systems, which are as follows: reconfiguration and restoration of the ship power system [35,36], optimization of the ship's power configuration under several operating conditions [37] and operational cost and emission minimization [19,38]. 
However, the implementation of meta-heuristic optimization for short-haul hybrid ferries with a battery storage system is limited.

\subsection{Motivation}

The trend toward further electrification of ferries is limited by the operational profiles of vessels and the optimization of the system. The introduction of multi-generation sources, storage systems, and interfacing power electronic converters in hybrid- and all-electric ferries increases the complexity of the system. Effective and efficient management of such a system to achieve the optimal use of available energy is ineffective with traditional approaches. Therefore, advanced optimization strategies to maximize the fuel savings and to minimize the operating costs while satisfying all operational constraints can help attract attention from ferry owners and operators to invest in hybrid- and all-electric ferries for increasing the trend of their use.

\subsection{Aim and contributions}

The present study aims to develop an innovative hybrid metaheuristic algorithm-based PMS for the fuel savings of short-haul ferries with hybrid power system. This study is the continuation of previous study [8]. The previous study suggests the use of single meta-heuristic optimization provides more fuel savings than the classical deterministic rule-based method for short-haul electric ferries. This paper proposes and addresses the performance improvement of using hybrid meta-heuristic over single metaheuristic algorithm to achieve more fuel savings for short-haul electric ferries. The proposed hybrid algorithm is based on fuzzy logic-grey wolf optimization (FL-GWO). Fig. 1 shows the structure of the proposed FL-GWO-based PMS. The main objective function of the optimization problem is to reduce the operating cost (OC) by minimizing the fuel consumption. The optimization problem is decomposed into two subproblems: (1) finding the best battery charging power based on battery state of charge (SoC) and power difference between generation and load and (2) determining the optimum power management for generators and battery. The performance of the proposed algorithm is compared with GWO and assessed based on fuel consumption, OCs, and standard deviation (SD). For this study, a measured load profile from an existing shorthaul ferry is used. The main contribution of this paper is summarized as follows:
- A multi-objective optimization PMS for the hybrid power system in electric ferries is designed to reduce the fuel consumption and OC of such a system;

- An innovative hybrid meta-heuristic algorithm (FL-GWO) is proposed, and its performance is compared with that of GWO;

- Optimal exploitation of battery energy storage system and generation units.

\subsection{Organization of the paper}

This paper is organized as follows: Section 2 describes the problem formation, objective function, and optimization constraints. Section 3 introduces the proposed algorithm used for the PMS. Section 4 discusses the case study and ferry specification. Section 5 presents the simulation results with associated analysis and discussions. Section 6 draws the conclusions on the performance of the proposed method.

\section{Problem formulation}

The integration of a battery storage system into short-haul ferries can reduce the fuel consumption and OC of the system. However, defining the main optimization parameters that can affect the fuel consumption of onboard engines is important. The problem of finding the optimal operation of the system is related to the scheduling and dispatching for generation units and battery storage system. The efficient operation of diesel generators coupled with prime movers, as the main sources of electrical energy onboard, is essential for achieving minimum OCs. Therefore, the output powers of generators are directly related to the main optimization problem. In addition, charging the battery from onboard generators cause additional load on generators. As a result, the battery also affects the output powers of generators. Hence, an established relation among these parameters is essential to form the optimization objective function. In this context, the OC function should incorporate the fuel consumption factor to identify the effect of each optimization parameter on the OC function.

\subsection{Fuel consumption factor}

The fuel consumption factor is incorporated in the OC function to assess the performance of the proposed PMSs. The specific fuel oil consumption (SFOC) is used to estimate the fuel consumption of onboard engines and measure the fuel efficiency of prime movers

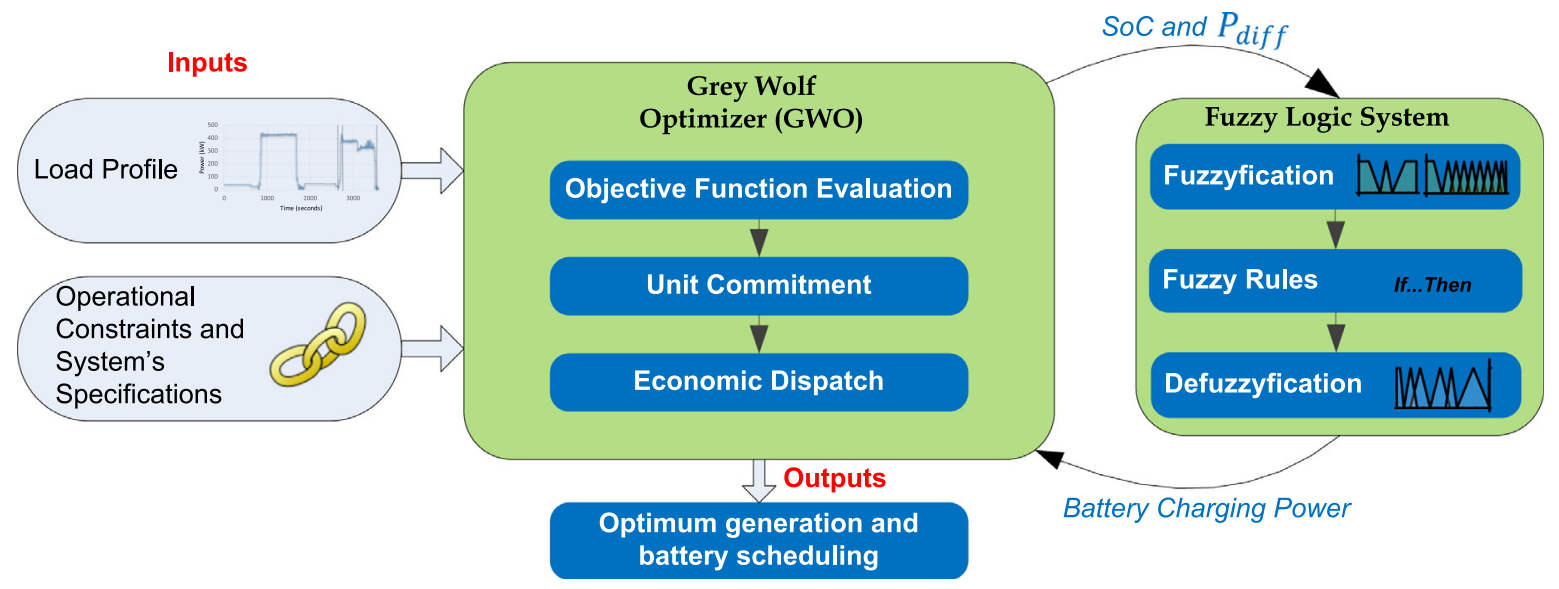

Fig. 1. Structure of the proposed PMS. 
that burns fuel to produce energy $[39,40]$. The SFOC equation relates the engine load (EL; ratio of generator output power at a specific point to its rated power) to fuel consumption at the same specific point. Thus, the SFOC equation can be used to identify the optimum operating region of a given engine and thereby take measures to improve the fuel consumption [39,41].

Fuel consumption data from different engine manufactures were used to form the SFOC equation for the ferry engine. All engines have the same rated power as the one used in the examined ferry. The purpose of using several engine data is to derive a further general equation that can be used for different diesel engines with similar rated power.

Fig. 2 shows the SFOC curves of a $320 \mathrm{~kW}, 400 \mathrm{kVA}$ diesel generator set for three manufacturers: Cummins [42], Caterpillar [43] and Perkins [44]. By using regression estimation, the seconddegree polynomial equations for SFOC considering Cummins, Caterpillar, and Perkins engines and an average of the three engines are as follows:

SFOC $_{\text {Cumm }}=0.169 x^{2}-0.292 x+0.392$

SFOC $_{C A T}=0.096 x^{2}-0.2 x+0.362$

$S F O C_{\text {Perkins }}=0.048 x^{2}-0.1 x+0.306$

$S F O C_{A v e}=0.106 x^{2}-0.201 x+0.355$

where $\mathrm{x}$ represents the relative engine load (EL) from 0 to 1 .

SFOC $=0.106 x^{2}-0.201 x+0.355$

As it can be seen from Fig. 2, the optimum operating region of all engines is above $70 \%$ engine load. Therefore, operating the engine within this range will significantly contribute to the reduction of fuel consumption.

\subsection{Optimization function and constraints}

Minimizing the OC of the ferry is the main optimization goal. The $\mathrm{OC}$ function consists of the fuel consumption equation. The main objective function of optimization is as follows:

Minmize OC $=\left[\sum_{t=1}^{T} S F O C_{E L, t} \times P_{E L, t}\right] \times T \times D_{\text {price }}$

where $O C$ is the operating costs (AUD\$), ELdenotes the engine load at the th time, $S F O C_{E L, t}$ represents the $S F O C$ at a specified engine

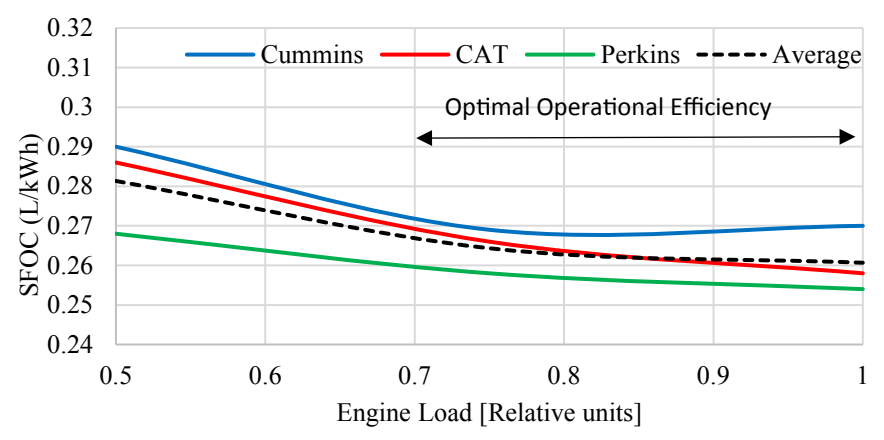

Fig. 2. SFOC curve for Cummins engine, CAT engine, Perkins engine and the average curve of all engines. Therefore, the derived quadratic equation for the SFOC can be expressed as. load (L/kWh), $P_{E L, t}$ indicates the instantaneous generated power at a specified engine load $(\mathrm{kW}), \mathrm{T}$ is the total time of one round trip in minutes and $D_{\text {price }}$ is the diesel price (AUD\$/L).

The optimization objective function is subjected to the following technical and operational constraints:

- Generator power limit:

The generator power limit constraint is applied to maintain the operation of the generator within the allowable limit:

$P_{n}^{\min } \leq P_{n} \leq P_{n}^{\max }$

where $P_{n}$ is the power generated by the $n$-th generator $(\mathrm{kW})$ and $P_{n}^{\min }$ and $P_{n}^{\max }$ are the minimum and maximum power limits of the generators $(\mathrm{kW})$, respectively.

- Power balance:

The power supplied from the generation side must be equal to the load demand for any period $t$,

$\sum_{t=1}^{\mathrm{T}} \sum_{n=1}^{\mathrm{N}}\left(P_{n, t} \times g_{t n}+P_{B, t}\right)=P_{L, t}$

where $P_{L, t}$ is the load power at th time $(\mathrm{kW}), P_{n, t}$ is the power generated by $n$-th generator at th time, $g_{\text {tn }}$ denotes the generator operating variable ( 0 is "OFF" and 1 is "ON"), and $P_{B, t}$ represents the battery power at the th time $(\mathrm{kW}) . P_{B, t}$ is either charging (negative value) or discharging (positive value), depending on the operating condition of the ferry (low or high demand).

- Blackout prevention:

Backout prevention constraint is applied for optimization to safeguard the system operation, thus ensuring that the maximum power at the generation side is sufficient to supply the maximum load power:

$N_{g} \times g_{\text {tn }} \times P_{n}^{\max }+P_{B, t}^{\max }-P_{L, t} \geq 0$

where $N_{g}$ is the number of generator units.

\section{- Battery energy storage system:}

The battery pack is operated in discharging mode at a low-load condition and charging mode at a high-load condition (when generators are operated at high load). Generally, a low-load condition refers to when the ferry is at the terminal, and a high-load condition refers to when the ferry is cruising. Therefore, the number of cruising periods and stops can influence the size of the battery pack. In addition, the depth of discharge (DoD) must be

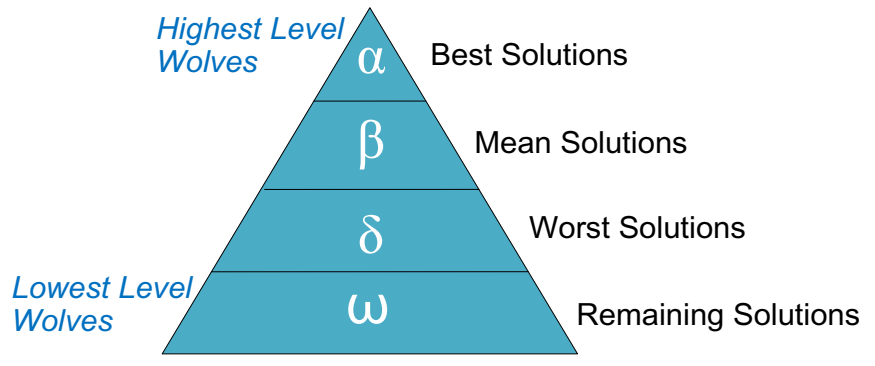

Fig. 3. Social hierarchy and structure of GWO. 
Table 1

Fuzzy expert system rules for battery charging power.

\begin{tabular}{llll}
\hline \multirow{2}{*}{ SoC } & \multicolumn{2}{l}{$P_{\text {diff }}$} & \\
\cline { 2 - 4 } & Low (L) & Average (A) & High (H) \\
\hline Critical (CR) & $\mathrm{EH}^{\mathrm{a}}$ & $\mathrm{EH}$ & $\mathrm{EH}$ \\
Lower Small (LS) & $\mathrm{EH}$ & $\mathrm{EH}$ & $\mathrm{EH}$ \\
Small (S) & $\mathrm{EH}$ & $\mathrm{EH}$ & $\mathrm{VH}^{\mathrm{b}}$ \\
Upper Small (US) & $\mathrm{EH}$ & $\mathrm{VH}$ & $\mathrm{H}$ \\
Lower Average (LA) & $\mathrm{VH}$ & $\mathrm{H}$ & $\mathrm{A}$ \\
Average (A) & $\mathrm{A}^{\mathrm{d}}$ & $\mathrm{A}$ & $\mathrm{A}$ \\
Upper Average (UA) & $\mathrm{A}$ & $\mathrm{A}$ & $\mathrm{L}^{\mathrm{e}}$ \\
High (H) & $\mathrm{L}$ & $\mathrm{L}$ & $\mathrm{L}^{\mathrm{g}}$ \\
Very High (VH) & $\mathrm{VL}^{\mathrm{f}}$ & $\mathrm{VL}$ & $\mathrm{SB}^{\mathrm{g}}$ \\
Full (FU) & $\mathrm{SB}$ & $\mathrm{SB}$ & $\mathrm{SB}$ \\
\hline
\end{tabular}

a Extreme High.

b Very High.

${ }^{c}$ High.

d Average.

e Low.

${ }^{f}$ Very Low and.

g Standby.

considered while sizing the battery. The DoD is an important parameter as it is directly related to the cycle life of batteries. Marine battery manufacturers recommend an $80 \%$ DoD to ensure a reasonable lifetime for batteries [8]. Therefore, the sizing of the battery system is calculated using the following equation:

$B_{\text {size }}=\frac{(2-D o D) \cdot\left(N_{S} E_{T}^{\max }\right)}{\eta_{d} \cdot N_{C}}$

where $B_{\text {size }}$ is the energy capacity of the battery pack in $\mathrm{kWh}, N_{S}$ is the number of stops per one round trip, $N_{C}$ is the number of cruising periods per one round trip, $E_{T}^{\max }$ denotes the highest energy at one terminal in $\mathrm{kWh}$, DoD is the designed DoD of the battery, and $\eta_{d}$ is the discharge efficiency of the battery.

The battery power equation consists of charging and discharging powers. The power equation of the battery is presented as follows:

$P_{B, t}=P_{\text {dcha }, t} \times\left(1-b_{t}\right)-P_{\text {cha }, t} \times b_{t}$

$$
\begin{aligned}
& P_{d c h a, t}=\frac{P_{L, t}}{\eta_{d c h a}} \\
& P_{\text {cha }, t}=\left(1-\frac{E_{B, t}}{E_{B}^{\max }}\right) \times P_{\text {cha }}^{\max } \times \eta_{\text {cha }}
\end{aligned}
$$

where $P_{d c h a, t}$ and $P_{\text {cha, } t}$ are the discharging and charging powers of the battery $(\mathrm{kW})$ at th time, respectively; $P_{d c h a}^{\min }$ and $P_{d c h a}^{\max }$ represent the minimum and maximum discharging powers $(\mathrm{kW})$, respectively; $P_{\text {cha }}^{\min }$ and $P_{c h a}^{\max }$ are the minimum and maximum charging powers (kW), respectively; $\eta_{d c h a}$ and $\eta_{c h a}$ are the discharging and charging efficiencies of the battery, respectively; $E_{B, t}$ is the battery energy at the th time (kWh), $E_{B}^{\max }$ is the maximum battery pack energy (kWh), $b_{t}$ is the battery operating variable ( 0 is "discharge" or 1 is "charge") and $P_{L, t}$ is the load power at th time.

The energy and the SoC values can be calculated by:

$E_{B, t}=E_{B, t-1}+\left[P_{d c h a, t} \times\left(1-b_{t}\right)-P_{c h a, t} \times b_{t}\right] \cdot \Delta t$

So $_{t}=\left(\frac{E_{B, t}}{E_{B}^{\max }}\right) \times 100 \%$

$D o D_{t}=1-S_{0} C_{t}$

where $S_{0} C_{t}$ is the SoC of the battery at th time and $D o D_{t}$ is the DoD of the battery at th time.

The power and SoC of the battery are operated within specified limits to ensure its reasonable lifetime. The battery operational constraints are as follows:

SoC ${ }^{\min } \leq \mathrm{SoC} \leq \mathrm{SoC}^{\max }$

$\left(1-\operatorname{SoC}^{\max }\right) \leq D o D \leq\left(1-\operatorname{SoC}^{\min }\right)$

$P_{B}^{\min } \leq P_{B} \leq P_{B}^{\max }$

$P_{d c h a}^{\min } \leq P_{d c h a} \leq P_{d c h a}^{\max }$

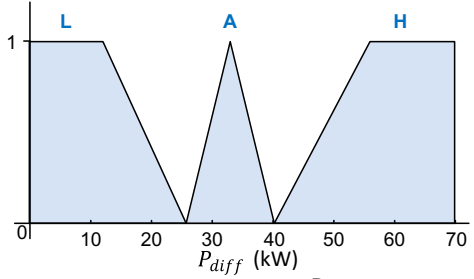

(a) Input variable $\left(P_{\text {diff }}\right)$

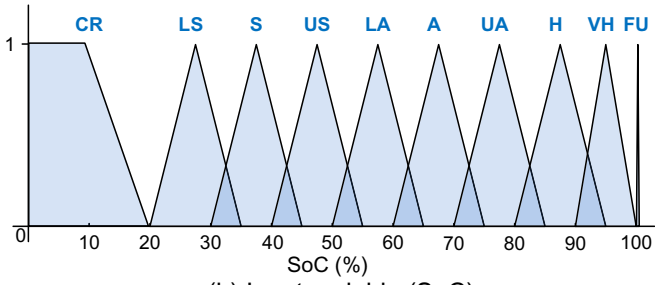

(b) Input variable (SoC)

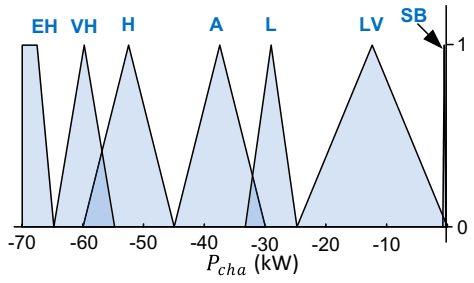

(c) Output variable $\left(P_{c h a}\right)$

Fig. 4. Fuzzifiers for (a) input variable $P_{\text {diff }}$,(b) input variable SoC and (c) output variable $P_{\text {cha }}$ 
$P_{\text {cha }}^{\min } \leq P_{\text {cha }} \leq P_{\text {cha }}^{\max }$

$E_{B}^{\min } \leq E_{B} \leq E_{B}^{\max }$

where $S O C^{\min }$ and $S O C^{\max }$ are the minimum and maximum SoC values of battery pack, respectively, and $E_{B}^{\min }$ and $E_{B}^{\max }$ are the minimum and maximum battery pack energy $(\mathrm{kWh})$, respectively.

\section{Power management strategy}

Several approaches are available for solving optimization problems on the basis of mathematical methods and metaheuristic algorithms [32]. Meta-heuristic algorithms are known for their high capability of solving multi-objective and complex optimization problems with several constraints in comparison with deterministic classical methods $[32,45]$. Despite the superior performance of these algorithms, several studies (as mentioned in the Introduction) have recommend using hybrid algorithms. The reason is that this combination assumes the advantage of the complementary characteristics between the algorithms to solve complex optimization problems with different linear and nonlinear constraints and provides further promising optimization results [29]. Therefore, a novel hybrid meta-heuristic method using FLGWO is proposed to solve the power management optimization of hybrid short-haul ferries.

\subsection{Grey wolf optimizer (GWO)}

GWO is a population-based meta-heuristic algorithm proposed in [46]. The implementation of GWO in different optimization problems provides competitive and promising performance and results compared with other evolutionary algorithms. For example, in hybrid power system optimization, GWO realizes higher performance and more promising results than genetic algorithm (GA) [47,48], harmony search (HA) [48], improved bat algorithm (IBA) [47], ant colony optimization [49], particle swarm optimization (PSO) $[46,47,50]$ and differential evolution [46].

GWO is inspired by the social behavior of grey wolves in pursuing, chasing, attacking, and hunting prey, where they perform social activities in groups of 5-12. The wolves are divided into four social groups: (1) leaders represented by alpha $(\alpha),(2)$ subleaders and represented by beta $(\beta),(3)$ wolves that obey the instructions from $\alpha$ and $\beta$ which represented by delta $(\delta)$, and (4) wolves that follow the instructions of higher-level wolves are represented by omega $(\omega)$. The $\alpha$ wolves are considered as the best solution because they belong to highest level of wolves and guide the hunting. Meanwhile, $\beta$ and $\delta$ wolves assist and follow the instruction from $\alpha$, and they are considered as the second-best (mean) and worst solutions, respectively. The $\omega$ wolves are considered as the other solutions. Fig. 3 presents the social hierarchy of grey wolves.

Grey wolves perform three steps in hunting as follows: (1) tracking and approaching, (2) encircling and pursuing, and (3) attaching the prey. The GWO algorithm mimics and represents this behavior mathematically as follows [46]:

$D^{\rightarrow}=\left|C^{\rightarrow} \cdot X_{p}(\mathrm{t})-\mathrm{X}^{\rightarrow}(t)\right|$

$X^{\rightarrow}(t+1)=X_{p}(t)-A^{\rightarrow} . D^{\rightarrow}$

where $D^{\rightarrow}$ is a calculated vector used to specify a new position of the wolf, $\mathrm{X}^{\rightarrow}$ is the position vector of the wolf, $X_{p} \overrightarrow{\text { represents the }}$ position of prey, and $A^{\rightarrow}$ and $D^{\rightarrow}$ are coefficient vectors calculated by Ref. [46]:

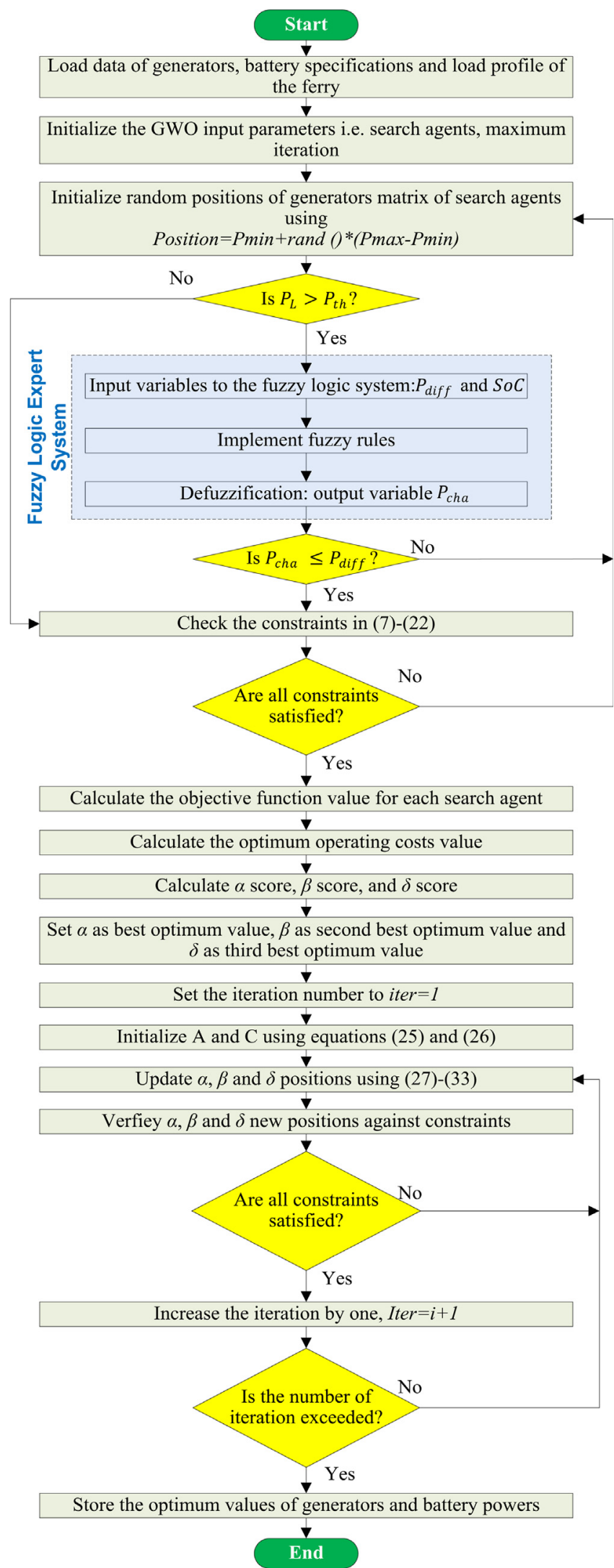

Fig. 5. Flowchart of the poroposed PMS based on FL-GWO 
Table 2

The specifications of the examined ferry.

\begin{tabular}{ll}
\hline Name & Bowen \\
\hline Capacity & 30 vehicles \\
Gen-sets & $2 \times 400 \mathrm{kVA}$ Cummings gen-sets \\
Travel distance and & $6.2 \mathrm{~km}$ for $60 \mathrm{~min}$ \\
$\quad$ duration (for round trip) & \\
Fuel Type & Diesel \\
Service speed & 7 knots \\
Number of stopes per round trip $\left(N_{S}\right)$ & 2 \\
\hline
\end{tabular}

$A^{\rightarrow}=2 a^{\rightarrow} \cdot r_{1}^{\rightarrow}-a^{\rightarrow}$

$C^{\rightarrow}=2 . r_{2}$

The search agents update their positions on the basis of the best search agents. Wolves $\alpha, \beta$, and $\delta$ calculate the possible positions of the prey while the simulation is running. The $\alpha$ wolves represent the best solution. Therefore, they are used as the final optimal solution. The new position vector of each wolf is calculated by the following equations [46]:

$D_{\text {Alpha }}^{\vec{a}}=\left|C_{1} \cdot X_{\text {Alpha }}-X^{\rightarrow}\right|$

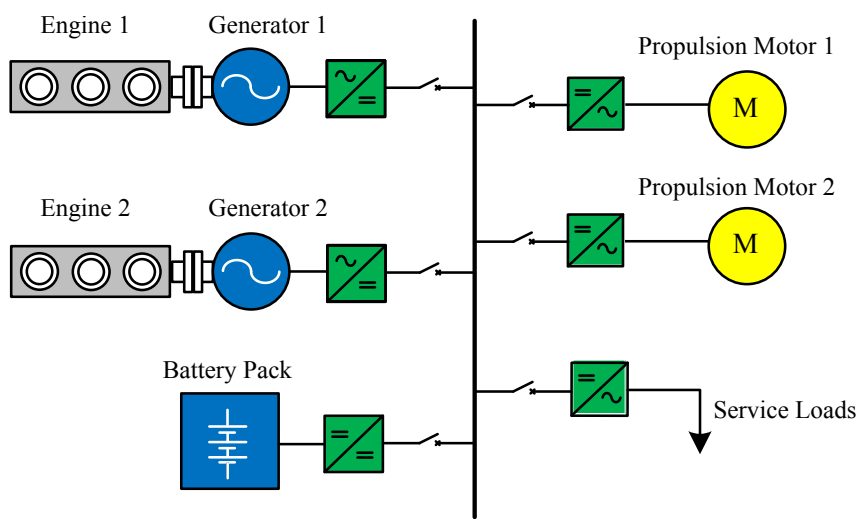

Fig. 6. Single-line diagram of the proposed hybrid system of the ferry.

$$
\begin{aligned}
& \overrightarrow{D_{\text {Beta }}}=\left|C_{2} \cdot X_{\text {Beta }}-X^{\rightarrow}\right| \\
& D_{\text {Delta }}=\left|C_{3} \cdot X_{\text {Delta }}-X^{\rightarrow}\right| \\
& X_{1} \overrightarrow{=}\left|X_{A l p h a}-A_{1} \cdot D_{A l p h a}\right| \\
& X_{2} \vec{a}=\left|X_{\text {Beta }}^{\overrightarrow{ }}-A_{2} \overrightarrow{D_{\text {Beta }}}\right| \\
& X_{3} \vec{a}=\left|X_{\text {Delta }}^{\overrightarrow{1}}-A_{3} \cdot D_{\text {Delta }}\right| \\
& X \rightarrow(t+1)=\frac{X_{1} \overrightarrow{+}+X_{2}+X_{3}}{3}
\end{aligned}
$$

where $\mathrm{X}_{\mathrm{Al}} \overrightarrow{p h a}, \mathrm{X}_{\mathrm{Beta}}$, and $\mathrm{X}_{\text {Delta }}$ represent the vectors of the grey wolf's positions; $\mathrm{D}_{\text {Alpha }}, \mathrm{D}_{\text {Beta }}$, and $\mathrm{D}_{\text {Delta }}$ are calculated vectors used to specify new positions of the wolf; and $X_{1}, X_{2}$, and $X_{3}$ indicate the position vectors of the wolves.

\subsection{Fuzzy logic expert system (FL)}

Nevertheless, fuzzy logic expert system is one of classical expert systems, it possesses advantage over other classical expert systems as it can handle multiple input variables with less complexity [32]. Moreover, in contrast to other intelligent systems, such as neural network, fuzzy logic systems do not require historical data and training. Furthermore, the fuzzy logic expert system uses various connotations, such as true, partially true, false, or partially false, thereby providing more flexibility and tolerant decision-making as regards the uncertainties of input variables than traditional binary logic methods with only two states (true or false) [51,52]. These advantages lead the fuzzy logic system to be an efficient controller option for the accurate selection of battery charging power for hybrid electric ferries.

The following are the three main process involved in the fuzzy system: fuzzification process of input variables, fuzzy rules, and defuzzification (output variables) [52]. In the first process (fuzzification), the controller accepts and maps crisp input variables into their membership functions on the basis of their membership degree. In the second process, the fuzzified inputs are sent to the interface engine, wherein the outputs are evaluated and calculated based on the fuzzy rules. In the third process (defuzzification), the

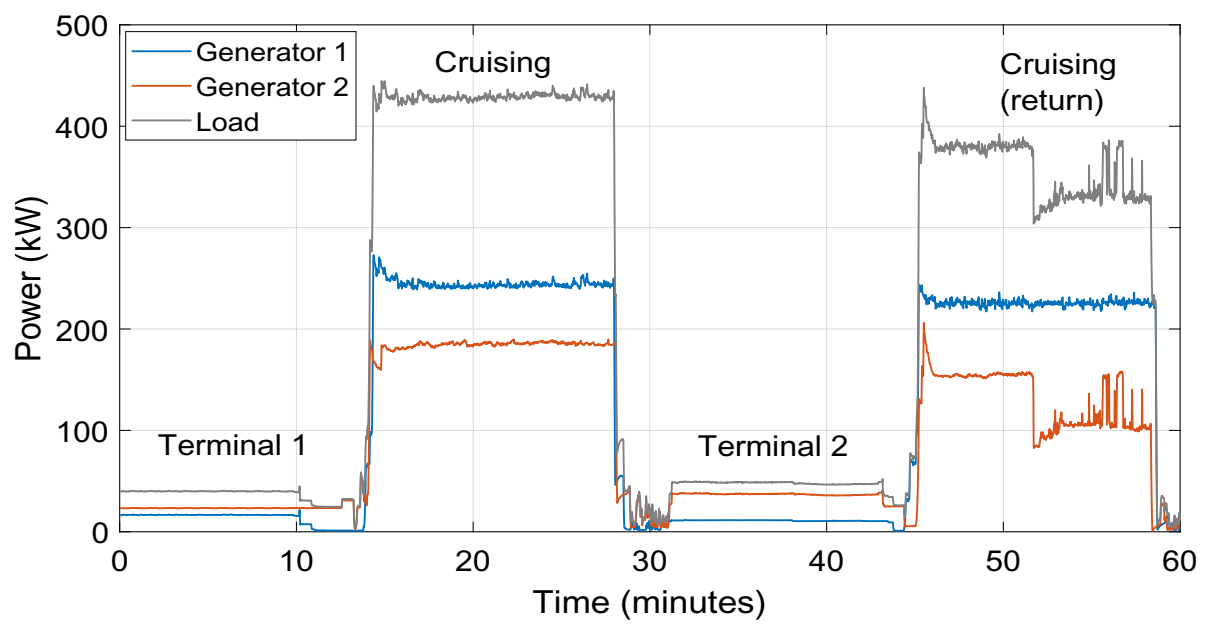

Fig. 7. Load profile of the ferry for a round trip: normal-load scenario. 


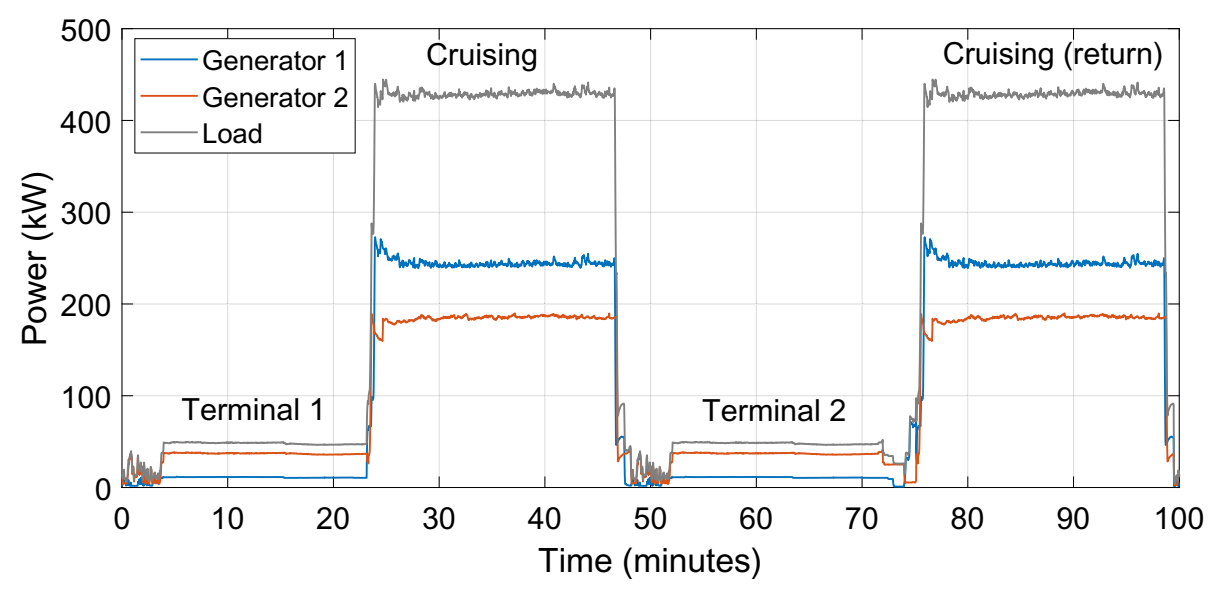

Fig. 8. Load profile of the ferry for a round trip: high-load scenario.

outputs are determined and converted to crisp values.

\subsection{Hybrid FL-GWO}

The proposed algorithm combines the fuzzy logic expert system with GWO. The proposed PMS aims to minimize the OC of the hybrid electric ferry. The PMS strategy is based on two rules: (1) Generators are out of operation with the battery set on discharge mode to supply the load demand when the ferry is at terminal (low-load demand condition). (2) Generators are operated with the battery set on charging mode when the ferry is cruising (high-load demand condition). Therefore, the main optimization parameters are the generator powers and the charging power of the battery.

The generators powers can be optimized by operating them above the low efficient region (above 25\% EL), which is close to the optimal operating region, thereby minimizing the SFOC and OC of the system. Charging the battery while cruising (when generators are operated) creates a correlation between the generator powers and the charging power of the battery. Therefore, the efficient management of charging the battery will contribute to SFOC minimization. In addition, the SoC/DoD of the battery will be maintained within the allowable limit, ensuring its safe operation and long life cycle.

Based on the power balance equation, the generation units must provide sufficient power to supply the load and charge the battery. According to the proposed PMS, this can occur when the ferry is cruising (not at the terminals). As GWO is a population-based algorithm, the generator power values are randomly generated. Consequently, the charging power values of the battery are also random. Therefore, the possibility of the battery not being charged to the required SoC at the end of the cruising period is increased. In addition, the battery might be fully charged before the end of the cruising period which will reduce the load on the generators. This may increase the possibility of operating generators far from the optimal SFOC region. A fuzzy logic expert system is developed and integrated with GWO to handle this issue and select the most appropriate battery charging power values to achieve a high optimization performance.

The fuzzy logic system is designed based on two input variables, namely, battery SoC and power difference $\left(P_{\text {diff }}\right)$ between the generator units and load demand. The output variable is the charging power of the battery $\left(P_{\text {cha }}\right)$. Table 1 shows the linguistic values of the input variables SoC and $P_{\text {diff }}$ and the linguistic values of the output variable $P_{\text {cha }}$. Fig. 4 depicts the membership functions used for the fuzzification.
The output variable $\left(P_{\text {cha }}\right)$ is fed into GWO. The value of $P_{\text {cha }}$ must be equal to the power difference between generation and load $\left(P_{\text {diff }}\right)$. If this condition is not satisfied, then the GWO generates a new set of values for generator powers and fed back the $P_{\text {diff }}$ and SoC to the fuzzy logic system. Fig. 5 shows the flowchart of the proposed FL-GWO power management strategy for hybrid electric ferry.

\section{Examined case study}

The proposed PMS is implemented on a short-haul electric ferry located in Bruny Island in Tasmania, Australia. The ferry, Bowen ferry, operates 6 days a week between the mainland of Tasmania and Bruny Island and performs seven round trips a day. The ferry load (propulsion and service loads) is powered by two $400 \mathrm{kVA}$ generator sets. Table 2 and Fig. 6 present the ferry specification and single-line diagram of the proposed hybrid system, respectively.

The measured load profile of the existing ferry for normal load demand is shown in Fig. 7 and for high load demand in Fig. 8.

As shown in Figs. 7 and 8, the ferry starts its trip from terminal 1, then stops at terminal 2 , and returns to terminal 1 . The time taken to perform one round trip is $60 \mathrm{~min}$. In the existing system, both diesel generators are operated at terminals. Hence, the prime movers are operated at a low load. This operating case results in further increase of fuel consumption as diesel engines are recommended to be operated at high engine load to ensure better fuel consumption efficiency.

Integration of battery storage system was proposed to address this issue. The battery is operated at terminals (at a low load) and charged while the ferry is cruising. The hybrid combination of

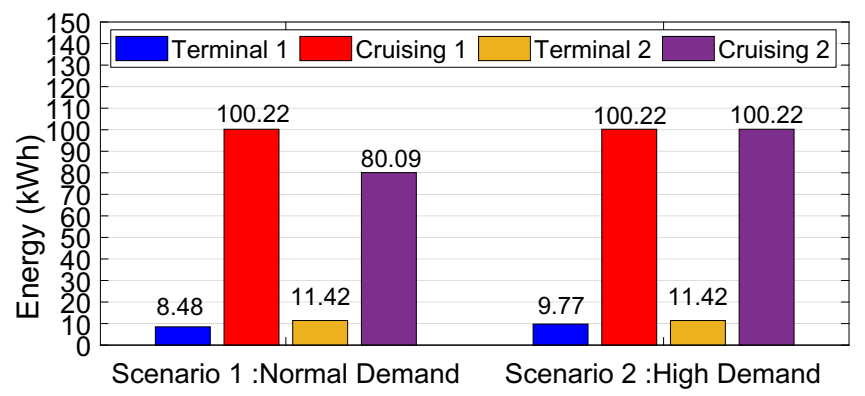

Fig. 9. Energy values at the terminals and while cruising for normal- and high-load scenarios. 
Table 3

Battery pack specification of the proposed system.

\begin{tabular}{|c|c|c|c|c|c|}
\hline $\begin{array}{l}\text { Number of modules in the } \\
\text { pack }\end{array}$ & $\begin{array}{l}\text { Voltage range of } \\
\text { pack }\end{array}$ & $\begin{array}{l}\text { Max current capacity of } \\
\text { pack }\end{array}$ & $\begin{array}{l}\text { Max charging power of pack } \\
\left(P_{\text {cha }}^{\max }\right)\end{array}$ & $\begin{array}{l}\text { Max discharge power of pack } \\
P_{d c h a}^{\max }\end{array}$ & $\begin{array}{l}\text { Pack energy } \\
\text { capacity }\end{array}$ \\
\hline 3 & $231-300 \mathrm{~V}$ & $75 \mathrm{Ah}$ & $76.5 \mathrm{~kW}$ & $135 \mathrm{~kW}$ & $14 \mathrm{kWh}$ \\
\hline
\end{tabular}

generators and battery storage system provides further fuel consumption even though the battery pack is charged from the same onboard generators. This case is as a result of the proper power management of the system. This study is an extension of [8] by proposing a new advanced PMS to achieve high fuel savings while satisfying all operational constraints.

Proper sizing of the battery is essential to ensure that the ferry can be operated in a safe and reliable way. The battery energy capacity is sized based on Equation (10). As shown in Fig. 9, the maximum energy at terminal $\left(E_{T}^{\max }\right)$ occurred in terminal 2 in both scenarios. Therefore, the battery energy capacity is selected as $14 \mathrm{kWh}$ in both scenarios.

The technical specification of the battery is obtained from Ref. [53]. Three modules of battery are selected for the proposed system based on the battery data sheet. Table 3 shows the battery pack specification. As high charging rate affects the battery's stateof-health [54-56], a 10\% reduction on the maximum charging power is applied to ensure an improved state-of-health for the battery pack. Therefore, the maximum charging power is set to $70 \mathrm{~kW}$.

\section{Results and discussions}

Simulation is performed for one round trip ( $60 \mathrm{~min})$. The performance of the proposed algorithm (FL-GWO) is compared with that of GWO and simulated for two load scenarios: normal and high loads. The parameters of the PMS are shown in Table 4.

The algorithm parameters are: number of agents $=30$, maximum number of iterations $=1000$ and problem dimensions $=3$. The simulation was performed using Matlab R2018b installed on 64-bit operating system, Intel ${ }^{\circledR}$ Core тм i5-6500 CPU at $3.20 \mathrm{GHz}$.

\subsection{Performance of the proposed PMS}

A total of 20 trial runs are performed for each method to examine the deviation of the optimal results in each method. The performance of each method is compared using three criteria over 20 different runs: (1) the best fuel consumption (F) of each trail, (2) the best operating cost $(\mathrm{OC})$ of each trial and (3) the standard deviation (SD) of the objective function values obtained in all trials.

The simulation results of both methods over 20 trials for normal- and high-load are shown in Table 5 and Table 6, respectively. In normal load scenario, the simulation results using GWO

Table 4

PMS parameters.

\begin{tabular}{|c|c|c|c|}
\hline Max. charging current $\left(P_{\text {cha }}^{\max }\right)$ & $70 \mathrm{~kW}$ & Min. battery pack capacity & $\left(E_{B}^{\min }\right) 2.8 \mathrm{kWh}$ \\
\hline Max. discharge current $\left(P_{d c h a}^{\max }\right)$ & $135 \mathrm{~kW}$ & Max. generator power $\left(P_{n}^{\max }\right)$ & $320 \mathrm{~kW}$ \\
\hline Max. value of SoC (SOC $\left.{ }^{\max }\right)$ & $100 \%$ & Number of generators $N_{g}$ & 2 \\
\hline Safe value of SoC (SOChigh $)$ & $90 \%$ & Max load power $\left(P_{L}^{\max }\right)^{\delta}$ & $640 \mathrm{~kW}$ \\
\hline Min. value of SoC $\left(\mathrm{SOC}^{\mathrm{min}}\right)$ & $20 \%$ & Charging $\left(\eta_{\text {cha }}\right)$ and discharging $\left(\eta_{d c h a}\right)$ efficiency & 0.98 \\
\hline Max battery pack capacity $\left(E_{B}^{\max }\right)$ & $14 \mathrm{kWh}$ & Power threshold $\left(P_{t h}\right)$ & $70 \mathrm{~kW}$ \\
\hline
\end{tabular}

Table 5

Optimization results for load scenario 1.

\begin{tabular}{|c|c|c|c|c|c|c|}
\hline \multirow[t]{2}{*}{ Run } & \multicolumn{3}{|l|}{ GWO } & \multicolumn{3}{|l|}{ FL-GWO } \\
\hline & $\mathrm{F}(\mathrm{L})$ & $\mathrm{OC}(\$)$ & SD & $F(L)$ & OC $(\$)$ & SD \\
\hline 1 & 56.41877176 & 79.26837432 & 0.253331434 & 54.8275023 & 77.03264073 & 0.05939153 \\
\hline 2 & 55.84418976 & 78.46108661 & 0.072816413 & 54.73828356 & 76.90728841 & 0.031361897 \\
\hline 3 & 56.25255046 & 79.0348334 & 0.201110095 & 54.55648294 & 76.65185852 & 0.02575396 \\
\hline 4 & 55.3640706 & 77.78651919 & 0.078021447 & 53.92881182 & 75.76998061 & 0.222947857 \\
\hline 5 & 54.63309333 & 76.75949613 & 0.307670785 & 54.57283606 & 76.67483466 & 0.020616341 \\
\hline 6 & 55.25861117 & 77.6383487 & 0.111153376 & 54.7556186 & 76.93164413 & 0.036808002 \\
\hline 7 & 56.1804744 & 78.93356653 & 0.178466137 & 54.5864866 & 76.69401367 & 0.016327783 \\
\hline 8 & 54.80534548 & 77.0015104 & 0.253554749 & 54.71876749 & 76.87986832 & 0.025230579 \\
\hline 9 & 56.13870877 & 78.87488582 & 0.165344729 & 54.82196425 & 77.02485977 & 0.057651655 \\
\hline 10 & 55.11881971 & 77.44194169 & 0.155071317 & 54.53801743 & 76.62591449 & 0.031555222 \\
\hline 11 & 54.80065935 & 76.99492638 & 0.255026979 & 54.82092286 & 77.02339661 & 0.057324482 \\
\hline 12 & 55.19419507 & 77.54784408 & 0.131390825 & 54.66576494 & 76.80539974 & 0.008578899 \\
\hline 13 & 55.43054896 & 77.87992129 & 0.057136103 & 54.57696191 & 76.68063148 & 0.019320131 \\
\hline 14 & 56.14586686 & 78.88494294 & 0.167593571 & 54.8321036 & 77.03910555 & 0.060837108 \\
\hline 15 & 56.60972075 & 79.53665765 & 0.31332141 & 54.73638111 & 76.90461546 & 0.030764209 \\
\hline 16 & 55.21954739 & 77.58346408 & 0.123425949 & 54.63607746 & 76.76368883 & 0.000747945 \\
\hline 17 & 56.19756165 & 78.95757412 & 0.183834397 & 54.70365903 & 76.85864094 & 0.020483993 \\
\hline 18 & 54.94832995 & 77.20240358 & 0.208633667 & 54.34520794 & 76.35501716 & 0.092129708 \\
\hline 19 & 56.34356551 & 79.16270954 & 0.229704069 & 54.7035665 & 76.85851093 & 0.020454923 \\
\hline 20 & 55.34364961 & 77.75782771 & 0.084437057 & 54.70374713 & 76.85876472 & 0.020511671 \\
\hline Mean & 55.61241403 & 78.13544171 & 0.176552225 & 54.63845818 & 76.76703374 & 0.042939895 \\
\hline
\end{tabular}


Table 6

Optimization results for load scenario 2 .

\begin{tabular}{|c|c|c|c|c|c|c|}
\hline \multirow[t]{2}{*}{ Run } & \multicolumn{3}{|l|}{ GWO } & \multicolumn{3}{|l|}{ FL-GWO } \\
\hline & $F(L)$ & OC $(\$)$ & SD & $F(L)$ & OC $(\$)$ & SD \\
\hline 1 & 59.86839632 & 84.11509683 & 0.095117189 & 58.59666487 & 82.32831414 & 0.020521896 \\
\hline 2 & 58.83541136 & 82.66375297 & 0.229413165 & 58.93807928 & 82.80800139 & 0.127783225 \\
\hline 3 & 59.75112083 & 83.95032476 & 0.058273035 & 58.58226591 & 82.3080836 & 0.015998208 \\
\hline 4 & 59.2673886 & 83.27068098 & 0.093699936 & 58.60868562 & 82.3452033 & 0.024298425 \\
\hline 5 & 59.57367923 & 83.70101932 & 0.002526644 & 58.4350327 & 82.10122094 & 0.030257688 \\
\hline 6 & 59.65659702 & 83.81751881 & 0.028576721 & 58.60248902 & 82.33649708 & 0.022351655 \\
\hline 7 & 59.52806881 & 83.63693668 & 0.01180267 & 58.4363452 & 82.10306501 & 0.029845342 \\
\hline 8 & 59.78902577 & 84.00358121 & 0.070181539 & 58.52841547 & 82.23242373 & 0.000919852 \\
\hline 9 & 59.28130391 & 83.290232 & 0.089328195 & 58.74576977 & 82.53780653 & 0.067365817 \\
\hline 10 & 60.02671953 & 84.33754093 & 0.144857203 & 58.21906677 & 81.79778881 & 0.098107175 \\
\hline 11 & 59.10855659 & 83.047522 & 0.143599799 & 58.5696977 & 82.29042527 & 0.012049686 \\
\hline 12 & 59.4711332 & 83.55694215 & 0.029689991 & 58.47869663 & 82.16256876 & 0.016539898 \\
\hline 13 & 59.98227444 & 84.27509559 & 0.130894 & 58.44491985 & 82.11511239 & 0.027151465 \\
\hline 14 & 59.83008733 & 84.0612727 & 0.083081747 & 58.51098036 & 82.20792741 & 0.006397397 \\
\hline 15 & 59.32925355 & 83.35760124 & 0.074263974 & 58.81269184 & 82.63183203 & 0.088390559 \\
\hline 16 & 59.71208531 & 83.89547987 & 0.046009343 & 58.39089842 & 82.03921228 & 0.044123246 \\
\hline 17 & 59.56992099 & 83.69573899 & 0.001345925 & 58.43646904 & 82.103239 & 0.029806437 \\
\hline 18 & 59.32464144 & 83.35112123 & 0.075712949 & 58.44446098 & 82.11446767 & 0.027295629 \\
\hline 19 & 59.90526654 & 84.16689949 & 0.106700617 & 58.41864476 & 82.07819589 & 0.035406247 \\
\hline 20 & 59.50180699 & 83.60003882 & 0.020053283 & 58.49191472 & 82.18114019 & 0.012387202 \\
\hline Mean & 59.56563689 & 83.68971983 & 0.076756396 & $\mathbf{5 8 . 5 3 1 3 4 3 3 7}$ & 82.23653744 & 0.036849852 \\
\hline
\end{tabular}

provide an optimal OC value of $\$ 76.7594$ with SD of 0.3076 . The average OC of all runs is $\$ 78.1354$ with an average SD of 0.1765 . On the other hand, the simulation results using FL-GWO provide better optimal OC value of $\$ 75.7699$ with a less SD value of 0.2229 than those using GWO. The average OC of all runs using FL-GWO is $\$ 76.7670$ with an average SD of 0.0429 . Similarly, in the high-load scenario, the FL-GWO provides a better optimal OC value of $\$ 81.7977$ than GWO with an optimal OC value of $\$ 82.6637$. In addition, FL-GWO provides a lower average SD than GWO.

As illustrated in Fig. 10, and Fig. 11, the optimal results obtained by GWO varies within wide range of optimal results set (between 76.7594 and 79.5366 in the normal-load scenario and between 82.6637 and 84.3375 in the high-load scenario). This case results a high average SD from the mean value. In contrast, results obtained by FL-GWO vary within a narrow range of optimal set results with boundaries from 75.7699 to 77.0391 in the normal-load scenario and from 81.7977 to 82.8080 in the high-load scenario. In other words, when random initial conditions are chosen for GWO and FLGWO, FL-GWO provide better global convergence as initial conditions are closer to the mean optimum solution than using GWO alone.

The best, mean, and worst results obtained from GWO and FLGWO for both load scenarios are shown in Table 7. Results obtained by FL-GWO method provide $1.29 \%$ and $1.05 \%$ improvements in minimizing OC compared with GWO in normal- and high-load scenarios, respectively. The worst optimal result had the highest improvement in both scenarios $(3.14 \%$ and $1.81 \%$ in normal- and high-load scenarios, respectively). Overall, FL-GWO's capability to ensure convergence to feasible solutions under different load conditions outperforms GWO. This case is apparent, as in all optimal solutions (best, mean, and worst), using the proposed FLGWO provides an improved optimal result compared with using GWO.

The best and worst optimal solutions for the total SFOC for both engines using FL-GWO and GWO are shown in Fig. 12 for normaland high-load scenarios. Both scenarios manifest an SFOC that is close to the optimal SFOC region using FL-GWO. This is expected to happen, as generator powers are often operated at high engine load in most of the time. Moreover, the optimal solutions provide a smooth transition from high engine load to zero when using FL-
GWO instead of GWO. This result can be observed at the end of the first cruising period, as the peak of SFOC curve in the best and worst optimal solutions obtained from GWO is attributed to the increase of total fuel consumption.

Fig. 13 shows the best solution of fuel consumption in liters for both engines using GWO and FL-GWO for (a) normal-load scenario and (b) high-load scenario. In Fig. 13 (a), normal-load scenario, the fuel consumption obtained by using GWO for engine 1 and 2 are $27.42 \mathrm{~L}$ and $27.20 \mathrm{~L}$ respectively. On the other hand, fuel consumption obtained by using FL-GWO for engine 1 and 2 are $25.33 \mathrm{~L}$ and $28.59 \mathrm{~L}$ respectively. In Fig. 13 (b), high-load scenario, the fuel consumption obtained by using GWO for engine 1 and 2 are $29.06 \mathrm{~L}$ and $29.77 \mathrm{~L}$ respectively. On the other hand, fuel consumption obtained by using FL-GWO for engine 1 and 2 are $29.11 \mathrm{~L}$ and $29.10 \mathrm{~L}$ respectively. It is apparent that FL-GWO provided less total fuel consumption than GWO by ensuring the convergence of fuel consumption solution of engine 1 to the most possible minimum solution in normal-load scenario. Similarly, in high-load scenario, fuel consumption solutions of both engines are optimized and converged to the most possible minimum solution.

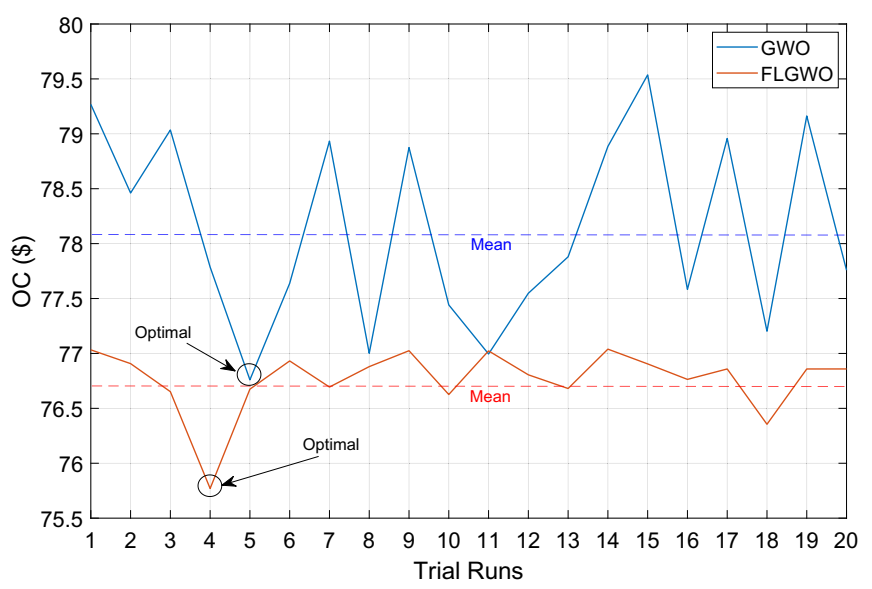

Fig. 10. Variation of OC optimal results over 20 runs for scenario 1 . 


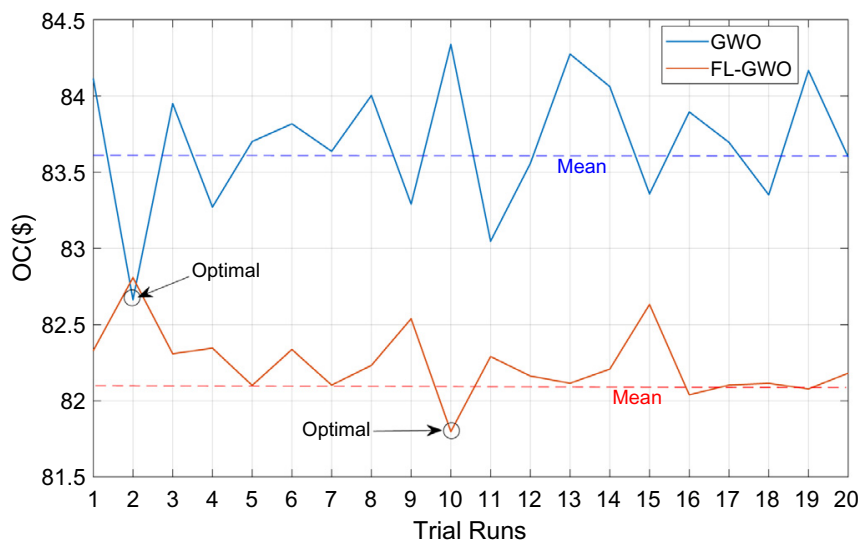

Fig. 11. Variation of OC optimal results over 20 runs for scenario 2.

\subsection{Sensitivity analysis}

In all aforesaid cases, GWO and FL-GWO methods satisfy the technical constraints and continuously fulfil the load demand. The main optimization parameters are generator 1 power $\left(P_{d g 1}\right)$, generator 2 power $\left(P_{d g 1}\right)$, and battery charging power $\left(P_{c h a}\right)$. The optimization parameters are optimized in the way that ensures both engines operate above the low operational efficiency region and close to the optimal SFOC region, resulting to reduced fuel consumption and OC. The influence of the abovementioned optimization parameters on the SFOC is discussed below.

\subsection{Effects of generators powers on SFOC}

Fig. 14 demonstrates the power produced by generators, battery power, and load for the two scenarios. Results suggest that both PMS methods are efficient in operating the ferry's power system in a safe and reliable way by satisfying the load demand and maintaining the battery power within the safe operational limits. As it can be seen, both generators are out of operation when the ferry is at the terminals and the battery operates in discharge mode to supply the load. In addition, both generators in the two scenarios are operated above the low efficiency region (above 25\% engine load).

As depicted in Fig. 2, the variation of engine load (generator output power) affects the SFOC value. The increase in power output of the generator reduces the SFOC until a certain value within the

Table 7

Best, mean, and worst optimal OC results.

\begin{tabular}{|c|c|c|c|c|c|c|}
\hline & \multicolumn{2}{|l|}{ GWO (\$AUD) } & \multicolumn{2}{|c|}{ FL-GWO (\$AUD) } & \multicolumn{2}{|c|}{ Improvement (\%) } \\
\hline & Normal Load & High Load & Normal Load & High Load & Normal Load & High Load \\
\hline Best & 76.75949613 & 82.66375297 & 75.76998061 & 81.79778881 & 1.29 & 1.05 \\
\hline Mean & 78.13544171 & 83.68971983 & 76.76703374 & 82.23653744 & 1.75 & 1.74 \\
\hline Worst & 79.53665765 & 84.33754093 & 77.03910555 & 82.80800139 & 3.14 & 1.81 \\
\hline
\end{tabular}

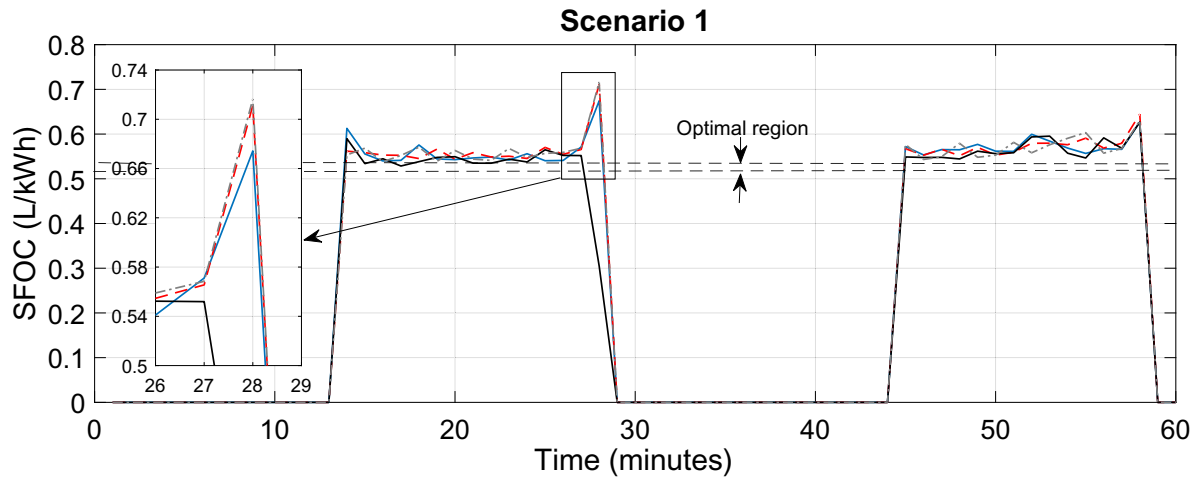

(a)

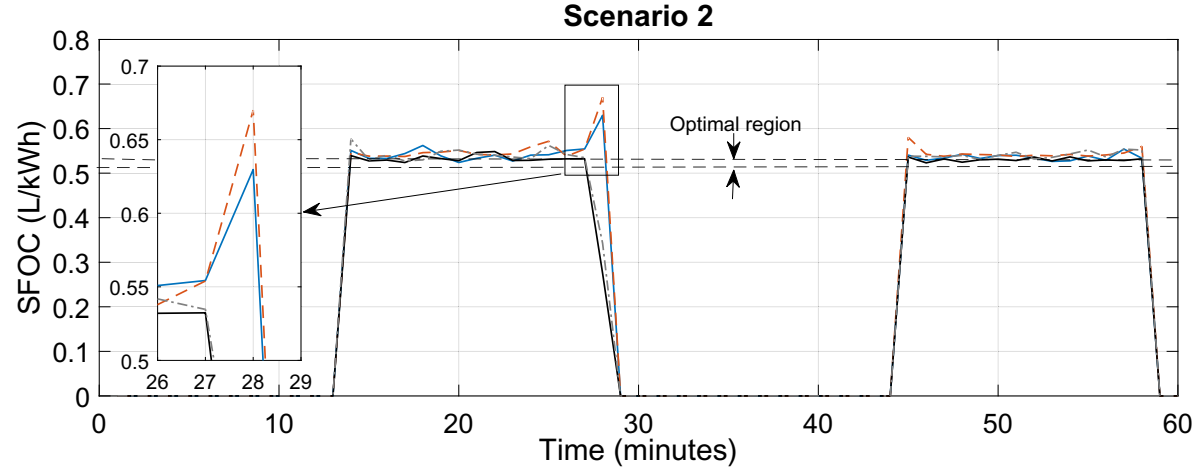

(b)

GWO Best - -- GWO Worst —_ FLGWO Best -.---FLGWO Worst

Fig. 12. SFOC results for scenarios 1 and 2 . 


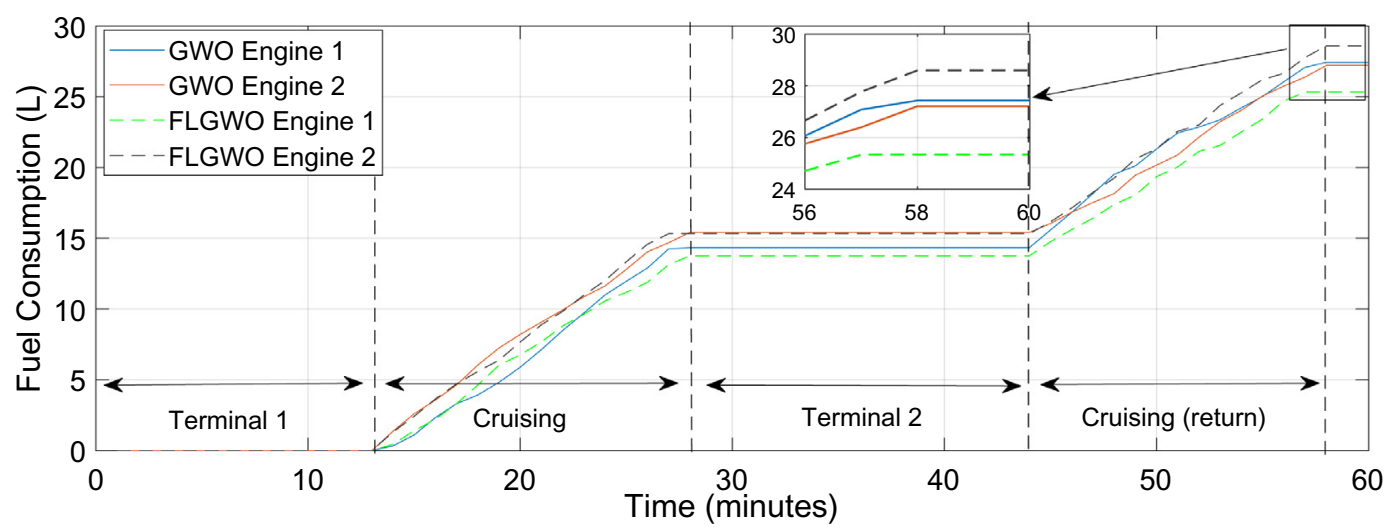

(a)

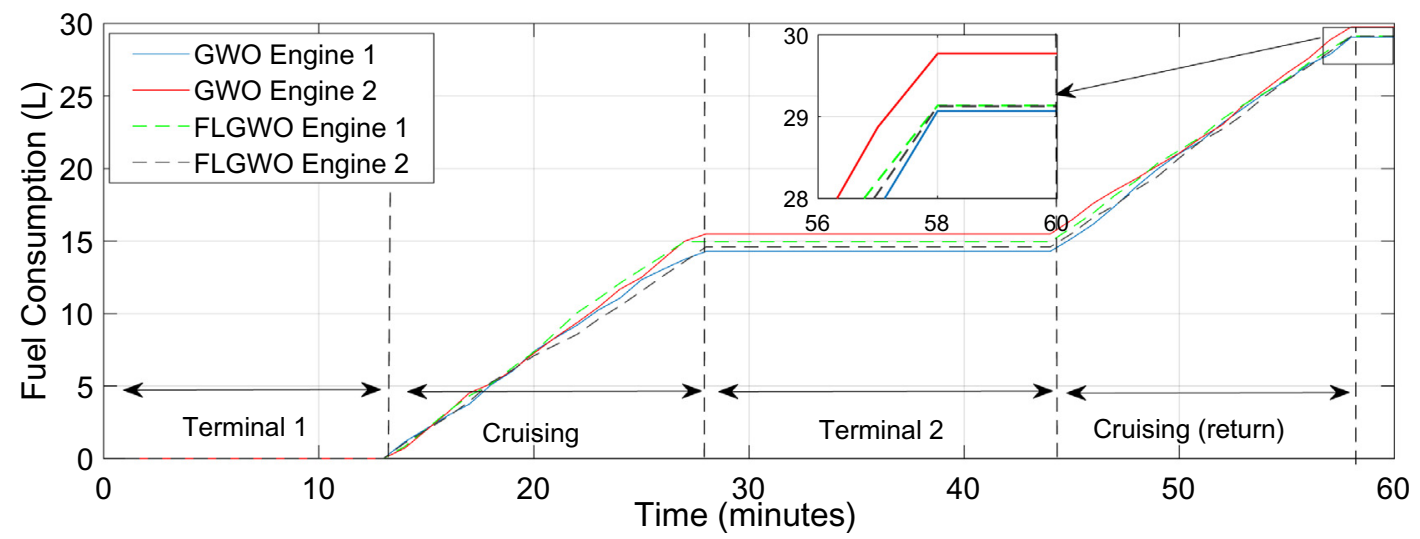

(b)

Fig. 13. Fuel consumption results for (a) normal-load secanrio and (b) high-load secanrio.

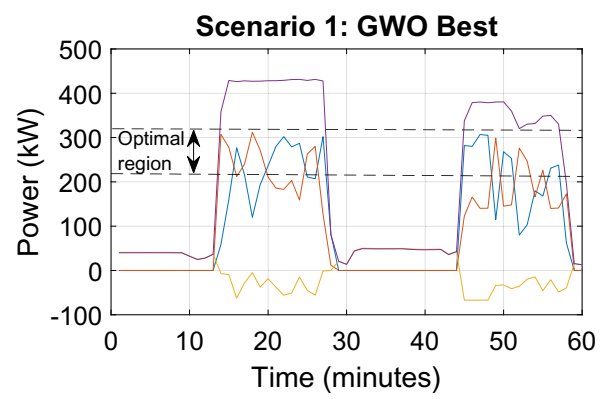

(a)

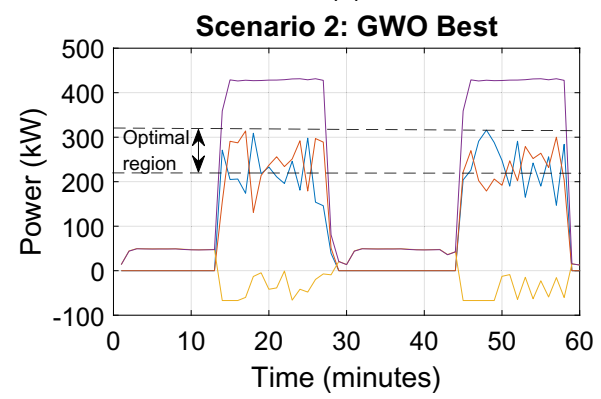

(c)

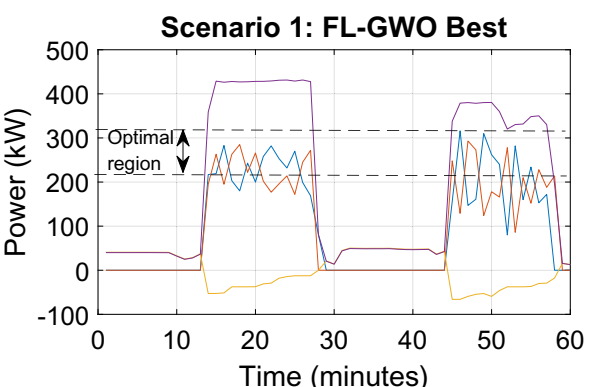

(b)

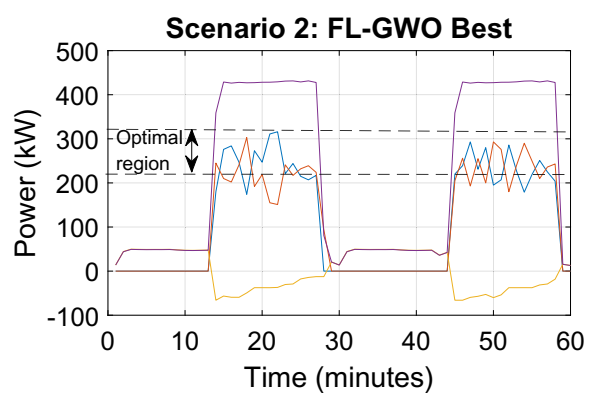

(d)

- Generator $1-$ Generator $2-$ Battery — Load

Fig. 14. Optimization output for generator and battery powers. 
optimal operational region. However, in some engines, the SFOC value keeps reducing until it reaches $100 \%$ engine load. This study uses an average SFOC equation, and an optimal range (i.e., from $70 \%$ to $100 \%$ engine load) is considered rather than optimal point.

Fig. 14 (a) and (b) show the simulation results for scenario 1 using GWO and FL-GWO. By using GWO, the variation of the generator powers is within a wide range. In this case, the possibility of both generators to operate far from the optimal operation region increases (far from the optimal SFOC region). Hence, this power deviation influences the OC as the SFOC is slightly increased. On the other hand, Fig. 14 (b) shows that FL-GWO provides less variation in generator powers than GWO. As it can be seen, the generator powers are operated close to the optimal region. The variation of generators powers is above $185 \mathrm{~kW}$ (above $56 \%$ engine load) in the first cruising period from $14 \mathrm{~min}$ to $28 \mathrm{~min}$. Hence, both generators are operated close to the optimal operational efficiency region (close to the optimal SFOC region), resulting in reduced SFOC and OC. Generator 1 has less operating time as its operation stopped $1 \mathrm{~min}$ before the end of the second cruising period even though the variation of powers in the second cruising period is higher than that in the first cruising period. This case also attributes to the reduction of SFOC and OC.

Fig. 14 (c) and (d) show the simulation results for scenario 2 using GWO and FL-GWO. It is apparent that the variation of generator powers using FL-GWO has less deviation from the optimal operational region than that using GWO. In the first cruising period, both methods provide a similar pattern of variation. However, FL-GWO is slightly closer to the optimal operational region (from $24 \mathrm{~min}$ to $28 \mathrm{~min}$ ) than GWO. FL-GWO provides less deviation in the second cruising period from the optimal region than GWO.

\subsection{Effects of battery SoC and power on SFOC}

Battery power is also an important parameter that can influence the SFOC value and OC function. Charging the battery from the onboard generators while the ferry is cruising adds extra load on the generators. Managing these extra loads (charging power) in a way that causes the generators to operate at or close to the optimal operational region will contribute in SFOC reduction. This approach can be performed by charging the battery until its $\mathrm{SoC}$ reaches a safe limit (above 90\%) in a uniform rate over the entire cruising period.

Fig. 14 shows that the battery is charged at a uniform and descending rate by using the FL-GWO method compared with the GWO method, where the battery charges at a random rate. This can be more depicted in Fig. 15 for normal- and high-load scenarios. In Fig. 15 (a), it is noted that for the best and worst optimal solution, the SoC/DoD is increased/decreased at a more uniform rate using FL-GWO than GWO. However, the SoC value reaches almost $100 \%$ at the end of the first cruising period (at $\mathrm{t}=28 \mathrm{~min}$ ) in all cases. Both methods successfully charge the battery up to the safe SoC region (above 90\%) at the end of the second cruising period. In Fig. 15 (b), at the end of the first cruising period, all optimal solutions using FLGWO and GWO can charge the battery up to the safe SoC region. It is noted that the worst optimal solution using GWO reaches to $100 \%$ SoC $1.5 \mathrm{~min}$ before the end of the cruising period, which changes the battery mode from charging to standby and reduces the load on engines. This case results in a slight increase of the SFOC and OC.

Overall, the battery power is optimized by charging the battery at a uniform SoC over the entire cruising period. Nevertheless, both methods have successfully optimized the battery power and charged the battery over the entire cruising period, FL-GWO

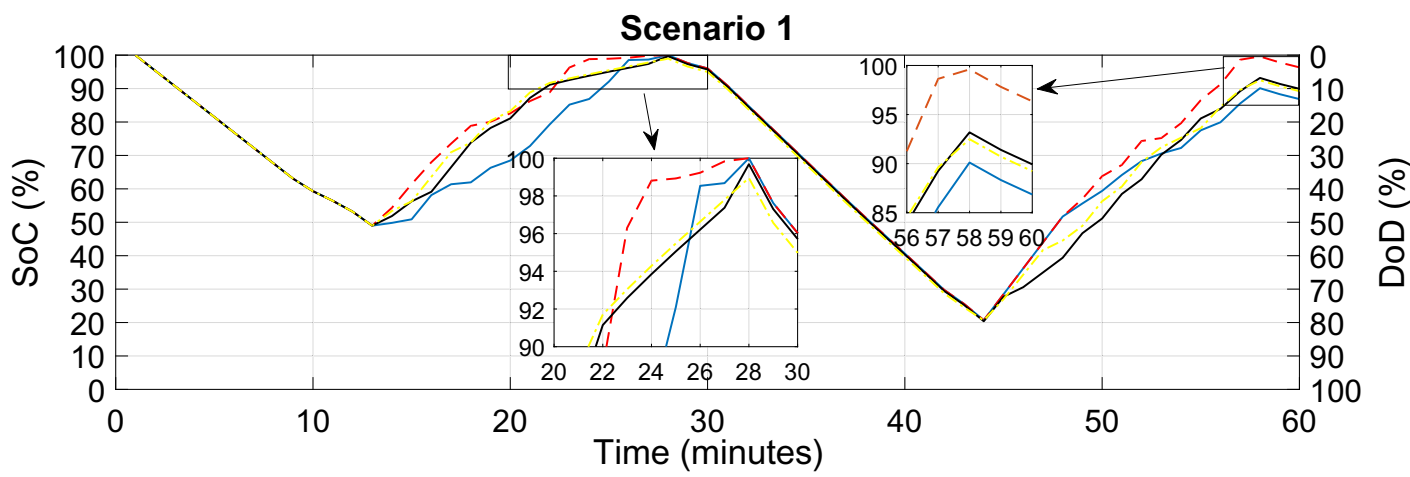

(a)

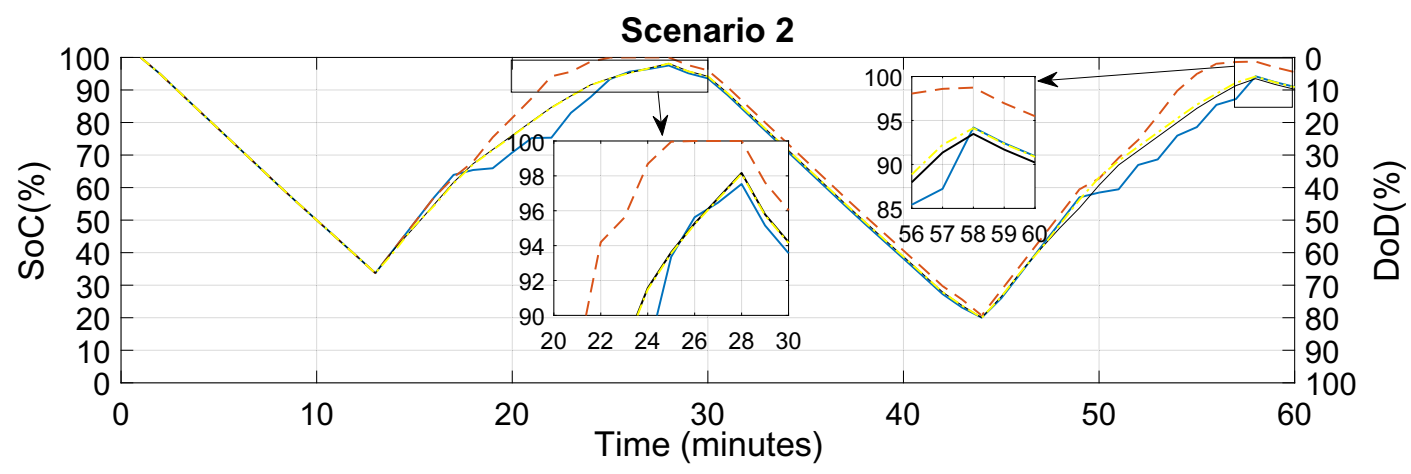

(b)

-GWO Best ---GWO Worst — FL-GWO Best_ FL-GWO Worst

Fig. 15. SoC of the battery for scenarios 1 and 2 . 


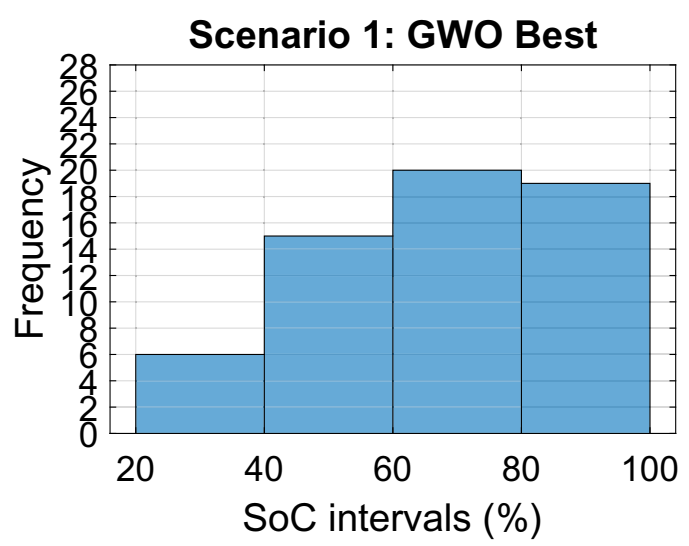

(a)

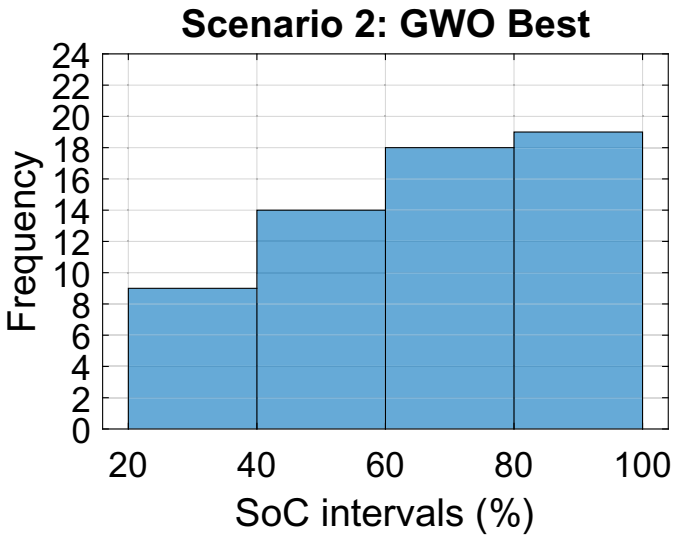

(c)

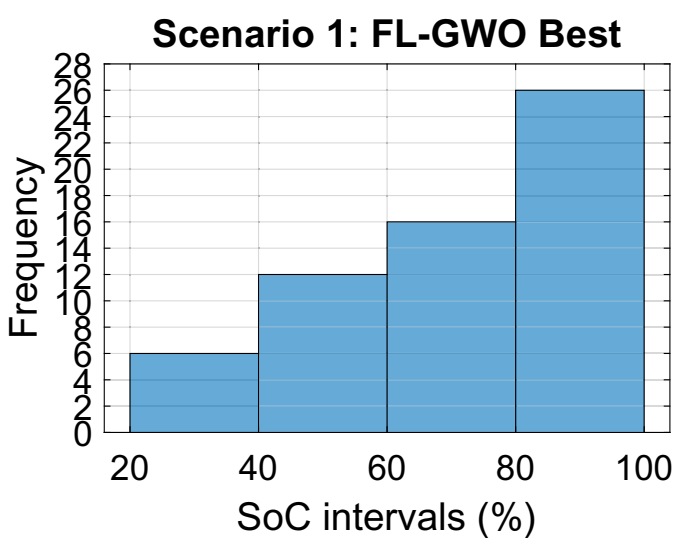

(b)

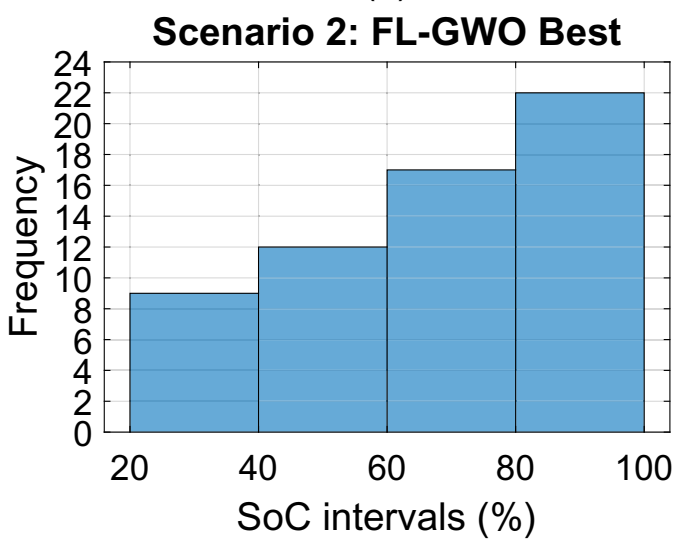

(d)

Fig. 16. Histogram of the SoC distribution for scenarios 1 and scenario 2 .

provides a further uniform charging rate. Fig. 16 shows the histogram of the SoC distribution for scenarios 1 and 2. The histogram presents the underlying frequency distribution of a set of SoC values within the complete round trip ( $60 \mathrm{~min})$. The $\mathrm{SoC}$ values are split into four intervals. In Fig. 16 (a) and (b), it is apparent that the number of times that the SoC value is above $80 \%$ is high with 26 occurrences (min) using the FL-GWO method. Thus, FL-GWO maintains the SoC above $80 \%$ for 26 min. By contrast, SoC values above $80 \%$ occur 19 times ( $\mathrm{min}$ ) using GWO. In the same pattern, for the high-load scenario, FL-GWO provides a higher frequency of SoC above $80 \%$ than GWO.

\section{Conclusions}

An effective power management method based on a hybrid algorithm is proposed for hybrid electric short-haul ferries with battery storage system. The hybrid algorithm is derived from an interactive approach based on FL-GWO for solving the optimization problem of minimizing operating costs (OC). The performance of the proposed PMS method is tested on the measured load data of a short-haul ferry under two operational scenarios and compared with that of GWO.

The key findings are summarized below:

- The capability of the proposed FL-GWO method to ensure convergence to feasible solutions under different load conditions is better than that of GWO.

- The proposed FL-GWO-based-PMS provides more promising results with less SD and fuel savings improvements of up to
$3.14 \%$ and $1.81 \%$ of the results in normal- and high-load scenarios, respectively, than GWO.

- The sensitivity analysis shows that the variation of generator powers using FL-GWO has less deviation from the optimal operational region (optimal SFOC region) than that using GWO. This is achieved by charging the battery power in a more uniform rate over the entire cruising period. Thus, the load on generators increases, thereby maintaining the highest engine performance over the entire cruising period. This practice attributes in reducing the SFOC and hence the OC.

Results of this study suggest that hybrid power systems with battery storage and hybrid meta-heuristic-based PMS is a good combination to achieve low fuel consumption for short-haul hybrid electric ferries. The insight gained from this study can also be of assistance to marine coastal vessels operating in close proximity to residential areas where both noise and air emissions are of concern as well as reducing the dependency on fossil fuels in ferry transportation industry.

Future work is required to incorporate real-time control methods with the hybrid optimization algorithm to adjust the operation of the lower-level devices. Moreover, incorporating the maintenance cost of the generator sets and battery system with the OC function can be considered in the overall assessment of the system.

\section{References}

[1] Hemmati R, Saboori H. Emergence of hybrid energy storage systems in 
renewable energy and transport applications-A review. Renew Sustain Energy Rev 2016;65:11-23.

[2] Jafarzadeh S, Utne IB. A framework to bridge the energy efficiency gap in shipping. Energy 2014;69:603-12.

[3] Trivyza NL, Rentizelas A, Theotokatos G. Impact of carbon pricing on the cruise ship energy systems optimal configuration. Energy; 2019.

[4] McCoy TJ. Electric ships past, present, and future [technology leaders]. IEEE Electrification Magazine 2015;3:4-11.

[5] Wen S, Lan H, Yu DC, Fu O, Hong Y-Y, Yu L, et al. Optimal sizing of hybrid energy storage sub-systems in PV/diesel ship power system using frequency analysis. Energy 2017;140:198-208.

[6] Mutarraf M, Terriche Y, Niazi K, Vasquez J, Guerrero J. Energy storage systems for shipboard microgrids-a review. Energies 2018;11:3492.

[7] Tang R, Wu Z, Li X. Optimal operation of photovoltaic/battery/diesel/coldironing hybrid energy system for maritime application. Energy 2018;162: $697-714$.

[8] Al-Falahi MD, Nimma KS, Jayasinghe SD, Enshaei H, Guerrero JM. Power management optimization of hybrid power systems in electric ferries. Energy Convers Manag 2018;172:50-66.

[9] Othman M, Anvari-Moghaddam A, Ahamad N, Chun-Lien S, Guerrero JM. Scheduling of power generation in hybrid shipboard microgrids with energy storage systems. In: 2018 IEEE international conference on environment and electrical engineering and 2018 IEEE industrial and commercial power systems Europe. EEEIC/I\&CPS Europe); 2018. p. 1-6.

[10] Zanne M, Počuča M, Bajec P. Environmental and economic benefits of slow steaming. Trans Marit Sci 2013;02:123-7.

[11] THORNTON J. Simply the best! Electric \& hybrid marine technology international. 2018. p. 42-6.

[12] M. Fuhrmann, A. Johnstone, A. Kühner, P. Lindemann, K. Tester, A. Wardwell, et al., "Maritime impact," DNV GL, Hamburg2018.

[13] DEIF, TYCHO BRAHE. HYBRID FERRY CASE STORY. In: Frisenborgvej 33, 7800 skive. Denmark: DEIF; 2018.

[14] Gagatsi E, Estrup T, Halatsis A. Exploring the potentials of electrical waterborne transport in Europe: the E-ferry concept. Transportation Research Procedia 2016;14:1571-80

[15] Holter M, Hodges J. The next ferry you board might run on batteries. Available, https://www.bloomberg.com/news/features/2018-03-13/the-next-ship-youboard-might-run-on-batteries; 2018.

[16] Kane M. Canada gets its first fully electric vessels: 2 massive ferries. Available, https://insideevs.com/canada-first-fully-electric-vessels/; 2018.

[17] CORVUS ENERGY SECURES CONTRACTS FOR FIVE ALL-ELECTRIC, BATTERYPOWERED FERRIES. Available, https://corvusenergy.com/corvus-energysecures-contracts-for-five-all-electric-battery-powered-ferries/; 2018.

[18] Boveri AP, Molinas M, Silvestro F, Skjong E. Optimal sizing of energy storage systems for shipboard applications. IEEE Transactions on Energy Conversion; 2018.

[19] Kanellos FD, Anvari-Moghaddam A, Guerrero JM. A cost-effective and emission-aware power management system for ships with integrated full electric propulsion. Electr Power Syst Res 2017;150:63-75.

[20] Han J, Charpentier J-F, Tang T. An energy management system of a fuel cell/ battery hybrid boat. Energies 2014;7:2799-820.

[21] Bassam AM, Phillips AB, Turnock SR, Wilson PA. An improved energy management strategy for a hybrid fuel cell/battery passenger vessel. Int J Hydrogen Energy 2016;41:22453-64.

[22] Bassam AM, Phillips AB, Turnock SR, Wilson PA. Development of a multischeme energy management strategy for a hybrid fuel cell driven passenger ship. Int J Hydrogen Energy 2017;42:623-35.

[23] Kalikatzarakis M, Geertsma R, Boonen E, Visser K, Negenborn R. Ship energy management for hybrid propulsion and power supply with shore charging Contr Eng Pract 2018;76:133-54.

[24] Lhomme W, Trovão JP. Zero-emission casting-off and docking maneuvers for series hybrid excursion ships. Energy Convers Manag 2019;184:427-35.

[25] Jin Z, Sulligoi G, Cuzner R, Meng L, Vasquez JC, Guerrero JM. Next-generation shipboard dc power system: introduction smart grid and dc microgrid technologies into maritime electrical netowrks. IEEE Electrification Magazine 2016;4:45-57.

[26] Al-Falahi M, Tarasiuk T, Jayasinghe S, Jin Z, Enshaei H, Guerrero J. AC ship microgrids: control and power management optimization. Energies 2018;11: 1458.

[27] Karadede Y, Ozdemir G, Aydemir E. Breeder hybrid algorithm approach for natural gas demand forecasting model. Energy 2017;141:1269-84.

[28] Gamarra C, Guerrero JM. Computational optimization techniques applied to microgrids planning: a review. Renew Sustain Energy Rev 2015;48:413-24.

[29] Frangopoulos CA. Recent developments and trends in optimization of energy systems. Energy 2018;164:1011-20.

[30] Al-Falahi MD, Jayasinghe S, Enshaei H. A review on recent size optimization methodologies for standalone solar and wind hybrid renewable energy system. Energy Convers Manag 2017:143:252-74.

[31] Khan FA, Pal N, Saeed SH. Review of solar photovoltaic and wind hybrid energy systems for sizing strategies optimization techniques and cost analysis methodologies. Renew Sustain Energy Rev 2018;92:937-47.

[32] Nabaei A, Hamian M, Parsaei MR, Safdari R, Samad-Soltani T, Zarrabi H, et al. Topologies and performance of intelligent algorithms: a comprehensive review. Artif Intell Rev 2018;49:79-103.

[33] Karadede Y, Özdemir G. A hierarchical soft computing model for parameter estimation of curve fitting problems. Soft Computing 2018;22:6937-64.

[34] Akdeniz F, Biçil M, Karadede Y, Özbek FE, Özdemir G. Application of real valued genetic algorithm on prediction of higher heating values of various lignocellulosic materials using lignin and extractive contents. Energy 2018;160:1047-54.

[35] Kankanala P, Srivastava SC, Srivastava AK, Schulz NN. Optimal control of voltage and power in a multi-zonal MVDC shipboard power system. IEEE Trans Power Syst 2012;27:642-50.

[36] Shariatzadeh F, Vellaithurai CB, Biswas SS, Zamora R, Srivastava AK. Real-time implementation of intelligent reconfiguration algorithm for microgrid. IEEE Transactions on sustainable energy 2014;5:598-607.

[37] Sciberras EA, Zahawi B, Atkinson DJ, Breijs A, van Vugt JH. Managing shipboard energy: a stochastic approach special issue on marine systems electrification. IEEE Transactions on Transportation Electrification 2016;2: $538-46$.

[38] Kanellos F. Optimal power management with GHG emissions limitation in allelectric ship power systems comprising energy storage systems. IEEE Trans Power Syst 2014:29:330-9.

[39] Jalkanen J-P, Johansson L, Kukkonen J, Brink A, Kalli J, Stipa T. Extension of an assessment model of ship traffic exhaust emissions for particulate matter and carbon monoxide. Atmos Chem Phys 2012;12:2641-59.

[40] Larsen U, Pierobon L, Baldi F, Haglind F, Ivarsson A. Development of a model for the prediction of the fuel consumption and nitrogen oxides emission trade-off for large ships. Energy 2015;80:545-55.

[41] Moreno-Gutiérrez J, Calderay F, Saborido N, Boile M, Valero RR, DuránGrados V. Methodologies for estimating shipping emissions and energy consumption: a comparative analysis of current methods. Energy 2015;86: 603-16.

[42] Cummins Available. https://powersuite.cummins.com/en/genset-model/ 88959.

[43] Caterpillar. Available, https://www.cat.com/en_AU/products/new/powersystems/electric-power-generation/diesel-generator-sets/1000029524.html.

[44] Engines P. Available, https://www.perkins.com/en_GB/products/new/perkins/ electric-power-generation/diesel/1000001903.html.

[45] Bukar AL, Tan CW. A review on stand-alone photovoltaic-wind energy system with fuel cell: system optimization and energy management strategy. J Clean Prod 1 June 2019;221:73-88.

[46] Mirjalili S, Mirjalili SM, Lewis A. Grey wolf optimizer. Adv Eng Software 2014:69:46-61.

[47] Nimma K, Al-Falahi M, Nguyen H, Jayasinghe S, Mahmoud T, Negnevitsky M. Grey wolf optimization-based optimum energy-management and batterysizing method for grid-connected microgrids. Energies 2018;11:847.

[48] Elahi A, Baygi SMH, Karsaz A. A novel framework for optimal sizing of hybrid grid-independent renewable energy system. In: Electrical engineering (ICEE). Iranian Conference on; 2018. p. 1292-7.

[49] Mirjalili S. How effective is the Grey Wolf optimizer in training multi-layer perceptrons. Appl Intell 2015;43:150-61.

[50] Sulaiman MH, Mustaffa Z, Mohamed MR, Aliman O. Using the gray wolf optimizer for solving optimal reactive power dispatch problem. Appl Soft Comput 2015;32:286-92.

[51] Adnan MM, Sarkheyli A, Zain AM, Haron H. Fuzzy logic for modeling machining process: a review. Artif Intell Rev 2015;43:345-79.

[52] Suganthi L, Iniyan S, Samuel AA. Applications of fuzzy logic in renewable energy systems-a review. Renew Sustain Energy Rev 2015;48:585-607.

[53] PBES. System specifications for the PBES power \& energy systems. Available, http://www.pbes.com/wp-content/uploads/2017/06/PBES_Power-Energy 2017-06-16.pdf; August 2017. 2017.

[54] Jiang J, Liu Q, Zhang C, Zhang W. Evaluation of acceptable charging current of power Li-ion batteries based on polarization characteristics. IEEE Trans Ind Electron 2014:61:6844-51.

[55] Fernández I, Calvillo C, Sánchez-Miralles A, Boal J. Capacity fade and aging models for electric batteries and optimal charging strategy for electric vehicles. Energy 2013;60:35-43.

[56] Gao Y, Jiang J, Zhang C, Zhang W, Jiang Y. Aging mechanisms under different state-of-charge ranges and the multi-indicators system of state-of-health for lithium-ion battery with Li (NiMnCo) O2 cathode. J Power Sources 2018;400: $641-51$. 


\section{Chapter 6: \\ Summary, Findings, Conclusions and Future Works}

This chapter provides a summary of the main findings and outcomes achieved in this study. The answers to the research questions, conclusions, and implications are further presented. Limitations of this research and recommendations for future work are discussed. 


\subsection{Summary}

The contributions with the associated approaches are summarized with respect to the guiding research questions.

\section{What types of optimization algorithms can improve the design and operation of hybrid power systems while fulfilling the reliability, economic, and environmental criteria?}

There is a limited literature that addresses the artificial optimizations and their performance in hybrid marine power systems. However, hybrid marine power systems have a lot in common with terrestrial hybrid power system; many of the methods and a lot of equipment and components at the generation side are same. Therefore, to answer this question, a comprehensive review is performed to classify the types of recent optimization methods and identify their performance assessment parameters for terrestrial hybrid power systems (Chapter 2 Part A).

The first step is to identify the assessment parameters of hybrid power system. These parameters evaluate the availability and feasibility of hybrid power system to help optimally design and operate the hybrid system. This step is done by categorizing the assessment parameters into four categories: economical, reliability, environmental, and social parameters.

The second step is to classify the type of optimization technique according to the performance in solving complex optimization problems. Therefore, the optimization algorithms are categorized as classical and modern. On the one hand, classical techniques use iterative, numerical, analytical, probabilistic, and graphical construction methods. These methods can solve single objective optimization problem. On the other hand, modern techniques use meta-heuristic and evolutionary algorithms that can solve complex optimization problems with several linear and nonlinear constraints.

Therefore, the types of optimization methods used in this study are classical, metaheuristic, and hybrid meta-heuristic, and their performance is assessed using economic, reliability, and environmental parameters.

What is the most feasible shipboard power system configuration in terms of reliability, ease of operation, and fuel efficiency? 
The advancements of architectures, control technologies, and power management strategies of ship microgrids are reviewed to answer this research question (Chapter 2 Part B).

In terms of power system configuration, the common shipboard power system architectures used in the last decade are analyzed. This study helps understand the common practices, component arrangements, voltage levels, and types of distribution system to select a suitable power system for this research.

In terms of operation and fuel efficiency, power management strategy plays a vital role in maintaining the power balance, improving fuel efficiency, preventing blackouts, and ensuring safe operation at various operating conditions. Thus, power management methods are categorized according to their objective function and operational constraints. This study helps understand the contributions of these methods to operate the system optimally and increase the fuel savings.

\section{What are the main parameters in sizing a battery storage system for electric ferries and how they affect the battery life?}

Sizing battery storage systems is important to achieve a reliable operation of the overall system and maintain the battery within reasonable lifetime. Many parameters, such as temperature, humidity, charge/discharge current, and operation cycle, can affect the battery lifetime. However, there are main factors that can significantly affect the size of the battery. To optimally size the battery, capacity of other components in the system needs to be determined. The size capacity can be changed depending on the operation and components of the entire system. Therefore, an approach is proposed to size the battery storage system for short-haul ferries (Chapter 3 Parts A and B).

The approach is derived on the basis of the maximum load and the recommended battery DoD. The size approach is tested on three different simulation scenarios of the ferry: (1) hybrid system with two diesel generator sets and onboard battery pack charged from onboard generator sets, (2) hybrid system with one diesel generator set and onboard battery bank charged from shore power supply, and (3) fully battery-powered ferry charged from the shore power supply.

A probability distribution method is used to investigate the effects of these parameters on the lifetime of the battery. A probability distribution provides the probabilities of occurrence 
of possible outcomes. In this method, one random variable is required (DoD value). This variable change randomly within specified boundaries in which a set of possible outcomes is generated.

How a power management strategy can optimize the operation of short-haul electric ferries in terms of fuel consumption, emission reduction, and cost effectiveness?

An efficient PMS is essential because it can optimally share power among all power system components of the ferry. The PMS also offers power saving, loading and unloading of generator sets, load-dependent start/stop, storage system control, and load sharing. This system can reduce the fuel consumption of onboard engines and their resulting emissions and operating costs of the systems.

To achieve this, three types of power management strategies are designed, and their performance is compared (Chapters 4 and 5). Load measurement and data collection of the existing diesel-electric short-haul ferry are used to validate the proposed PMSs. The proposed PMSs are applied to the hybrid power system configuration of the short-haul ferry. The three types of power management strategies are classical rule-based, meta-heuristic GWO, and hybrid FL-GWO methods.

Firstly, rule-based and GWO methods are designed and applied to the hybrid power system of short-haul ferry. The performance of both methods is assessed in terms of fuel consumption and emission. The fuel consumption factor consists of SFOC equation of onboard engine, which is derived using regression method from the data obtained from a specific engine manufacturer. The emission factor $\left(\mathrm{CO}_{2}, \mathrm{SO}_{\mathrm{x}}\right.$, and $\mathrm{NO}_{\mathrm{x}}$ emissions) is based on the equation obtained from Entec inventory with the top-down method because it covers the emission estimations from ferries and requires only the load profile of the engine to calculate the emissions. In rule-based method, several operating states based on battery SoC and total load power are defined to control the power sharing of each component. Outputs are the decisions to switch the generators on/off and charge/discharge the onboard batteries. In GWO, the main objective function of the optimization is to minimize the fuel consumption of onboard engines subjected to several operational constraints. The optimization parameters are the onboard generator powers and battery power. Optimizing these parameters will optimize the value of SFOC and results in reduction in fuel consumption and emission. 
Secondly, a novel hybrid meta-heuristic algorithm-based PMS is developed, and its performance is compared with that of GWO. The proposed hybrid PMS method applies an interactive approach based on a GWO and fuzzy expert system to improve the computational efficiency of the algorithm. The fuzzy logic system is integrated with GWO to select the most appropriate battery charging power values for achieving high optimization performance. The proposed FL-GWO method is applied and tested on two simulation scenarios: normal load scenario and high load scenario. The performance of both methods is assessed in terms of operating cost, fuel consumption, and standard deviation. The fuel consumption factor based on SFOC equation is incorporated in the operating cost function. A more general SFOC equation is derived, which can be used for different diesel engines with the same rated power.

\subsection{Findings}

The methods and findings of this thesis can be used by researchers and designers in the field of marine power system. This study is the first to develop a hybrid meta-heuristic algorithm and test other optimization strategies for power management optimization in short-haul hybrid electric ferries with a battery storage system. This study enables other researchers and designers to adapt and extend the methods to other marine vessels. The main findings and associated subfindings of this thesis are listed below:

1. Standalone hybrid system with DC distribution is the most suitable configuration for short-haul electric ferries among others.

- DC distribution system is an effective power system architecture when a battery storage system is incorporated into the marine power system.

- Battery-powered system in short-haul ferries is the most effective configuration in terms of operational cost only compared with standalone hybrid system and hybrid system with shore charging configurations.

- However, battery-powered system configuration followed by hybrid system configuration with shore charging possesses high investment costs due to the high capacity of onboard battery and cost of shore charging facility.

2. The main affecting parameters of the size of the battery for short-haul electric ferries are battery DoD and operational profile (number of stops at terminal, number of cruising periods, and maximum load). 
- A proper sizing of the onboard battery is important to achieve a safe and reliable operation of the system.

- Battery DoD significantly influences the size and thus the lifetime of the battery. This factor also affects the PBP of the system. Large DoD value results in low battery size capacity, small PBP, and short battery lifetime.

\section{Hybrid optimization algorithm-based PMS provides more promising results} compared to single optimization algorithm and classical optimization methods for short-haul electric ferries.

- The three power management strategies, namely, classical rule-based, metaheuristic GWO, and hybrid meta-heuristic FL-GWO, always operate the ferry power system safely by matching the generation with load demand.

- The three power management strategies provide $100 \%$ reductions of emissions produced by onboard engines at berth.

- The three power management strategies applied to the hybrid power system of the ferry provide lower fuel consumption than the existing diesel-electric system of the ferry.

- The GWO method can optimize the SFOC and provide higher fuel consumption reduction than using rule-based method.

- Rule-based method equally shares the load among onboard generators while GWO optimizes the generator powers efficiently by operating generators at high load and close to the optimal operational point.

- In terms of optimizing battery power, charging the battery while cruising (when generators are operated) creates a correlation between the generator powers and the battery charging power. Therefore, the efficient management of charging the battery while cruising will contribute to SFOC minimization, which in turn reduces the fuel consumption and operating cost.

- When GWO method is used, the battery is charged at low and fixed charging rate to operate the generators at high load over the entire cruising period. By contrast, given that rule-based method is set on the basis of predetermined conditions, the battery is charged at highest charging rate from the first moment 
of cruising period. By doing so, the battery becomes fully charged before the end of cruising period. This condition reduces the load on generators which in turn increases the SFOC.

- The proposed hybrid FL-GWO method can better ensure convergence to feasible solutions under different load conditions than GWO.

- The variation in generator powers using FL-GWO has lower deviation from the optimal operational region (optimal SFOC region) than that using GWO.

- FL-GWO provides lower deviation of optimal solutions (operating costs) than GWO. Therefore, FL-GWO provides more fuel consumption reductions than GWO.

\subsection{Conclusions and Implications}

Power management strategies for hybrid short-haul ferries are developed, and their performance is evaluated and compared using numerical simulation. Several system configurations with battery storage size calculation are investigated.

Overall, the battery integration into short-haul electric ferries can be considered an effective solution for fuel savings and emission reductions. This results a reduction of fuel consumptions of onboard engines because they are shut down at terminals and efficiently operated while cruising. This condition also reduces engine noises at terminals. In terms of power system architecture, the standalone hybrid system with DC distribution is considered an effective architecture when energy storage is incorporated into electric ferries.

In terms of power management strategies, a meta-heuristic optimization method provides better fuel consumption and emission reduction than classical methods. This performance is due to meta-heuristic algorithms effectiveness to solve complex optimization problems with several operational constraints to find the optimal solution, that is in contrast to classical methods that use pre-determined conditions. Despite the superior performance of the metaheuristic algorithm, the hybrid meta-heuristic algorithm provides further promising optimization results due to its high capability to converge to feasible solutions under different loading conditions.

Overall, these results suggest that DC distribution hybrid power system with battery storage and hybrid meta-heuristic-based power management strategy is an efficient combination to achieve low fuel consumption, reduction of emissions, and minimum noises at 
terminals for short-haul ferries. These outcomes and methods enable other researchers and designers in the field of marine power systems to adapt and extend the methods to other marine vessels. The insight gained from this study can also be of assistance to marine coastal vehicles operating in the close proximity to residential areas where both noise and air emissions are of concern as well as reduction on the dependency of fossil fuels in ferry transportation industry.

\subsection{Limitations and Future Works}

The power management strategies were developed to optimize the power system for short-haul ferries with minimal focus on improving local controllers of each component of the system. Although droop control was investigated and implemented in the model, further investigation and development of control methods are required. Thus, research incorporating and developing local controllers with each component can be conducted to improve the operation of the optimizer.

In this study, the proposed power management optimization problem is subjected to several technical and operational constraints and assessed based on economic, reliability, and environmental factors. However, recommendations for repetitive shutdown-restart of the engines should also be considered in the overall assessment of the proposed system. Moreover, incorporating the maintenance cost of the generator sets and battery system with the cost function can be considered in the overall assessment of the hybrid system.

This study compares battery-powered and diesel-electric driven ferries in terms of investment, operational, and maintenance costs. However, a study based on detailed economic and technical investigation should be conducted to distinguish the overall costs and lifetime of retrofitting current diesel-electric ferries with batteries and building new ferries incorporating batteries.

Given that short-haul ferries have short turnaround times at terminals, the batteries will be charged from shore power supply at high C-rates, thereby producing excessive heat. This excessive heat increases the temperature of batteries, which in turn reduces their performance and lifetime. Therefore, the methods of cooling and thermal management for batteries in battery-powered ferries should be investigated or considered as one of the technical constraints in the optimization process. 


\section{References}

[1] E. Skjong, R. Volden, E. Rødskar, and M. Molinas, "Past, Present, and Future Challenges of the Marine Vessel's Electrical Power System," IEEE TRANSACTIONS ON TRANSPORTATION ELECTRIFICATION, vol. 2, 2016.

[2] E. A. Sciberras, B. Zahawi, and D. J. Atkinson, "Reducing shipboard emissions Assessment of the role of electrical technologies," Transportation Research Part D: Transport and Environment, vol. 51, pp. 227-239, 2017.

[3] IMO. (2016). Low carbon shipping and air pollution control. Available: http://www.imo.org/en/MediaCentre/HotTopics/GHG/Pages/default.aspx

[4] Z. Jin, G. Sulligoi, R. Cuzner, L. Meng, J. C. Vasquez, and J. M. Guerrero, "NextGeneration Shipboard DC Power System: Introduction Smart Grid and dc Microgrid Technologies into Maritime Electrical Netowrks," IEEE Electrification Magazine, vol. 4, pp. 45-57, 2016.

[5] J. F. Hansen and F. Wendt, "Historyand Stateofthe Art in Commercial Electric Ship Propulsion, Integrated Power Systems, and Future Trends," Proceedings of the IEEE, vol. 103, pp. 2229 - 2242, 2015.

[6] E. K. Dedes, D. A. Hudson, and S. R. Turnock, "Assessing the potential of hybrid energy technology to reduce exhaust emissions from global shipping," Energy Policy, vol. 40, pp. 204-218, 2012.

[7] E. Skjong, E. Rødskar, M. M. Molinas Cabrera, T. A. Johansen, and J. Cunningham, "The marine vessel’s electrical power system: From its birth to present day," 2015.

[8] Z. Jin, L. Meng, J. M. Guerrero, and R. Han, "Hierarchical control design for a shipboard power system with DC distribution and energy storage aboard future more-electric ships," IEEE Transactions on Industrial Informatics, vol. 14, pp. 703-714, 2018.

[9] M. Weiming, "Development of vessel integrated power system," in 2011 International Conference on Electrical Machines and Systems, 2011, pp. 1-12.

[10] B. Zahedi and L. E. Norum, "Modeling and Simulation of All-Electric Ships With LowVoltage DC Hybrid Power Systems," IEEE TRANSACTIONS ON POWER ELECTRONICS, vol. 28, pp. 4525 - 4537, 2013.

[11] S. KNIGHT. (2018) Digital Age. Electric \& Hybrid Marine Technology International. $30-34$. 
[12] M. Fuhrmann, A. Johnstone, A. Kühner, P. Lindemann, K. Tester, A. Wardwell, et al., "Maritime Impact," DNV GL, Hamburg2018.

[13] K. Gallagher, A. Johnstone, A. Wardwell, and K. Tester, "Ferry and RO-RO Update," DNV GL, Hamburg, Germany2018.

[14] M. Zanne, M. Počuča, and P. Bajec, "Environmental and Economic Benefits of Slow Steaming," Transactions on Maritime Science, vol. 02, pp. 123-127, 2013.

[15] DEIF, "TYCHO BRAHE: HYBRID FERRY CASE STORY," ed. Frisenborgvej 33, 7800 Skive, Denmark: DEIF, 2018.

[16] J. THORNTON. (2018) Simply the best! Electric \& Hybrid Marine Technology International. 42 - 46.

[17] B. Maritime. (2018). HYBRID 600-PAX FERRY ENTERS SERVICE WITH SAN FRANCISCO-BASED RED AND WHITE FLEET. Available: https://www.bairdmaritime.com/work-boat-world/passenger-vessel-world/3309-hybrid600-pax-ferry-enters-service-with-san-francisco-based-red-and-white-fleet

[18] (2018, Dec 2018). Ben Woollacott. Available: http://www.lmgmarin.no/references/409/ben-woollacott

[19] (2018, Dec 2018). DAME VERA LYNN. Available: http://www.lmgmarin.no/references/410/dame-vera-lynn

[20] R. Moore. (2018, Dec 2018). Victoria of Wight: the benefits of battery propulsion. Available: https://www.passengership.info/news/view,ivictoria-of-wighti-the-benefitsof-battery-propulsion_54446.htm

[21] J. Taylor. (2017, Dec 2018). Seaspan Ferries Corporation Announces Arrival of Second New Liquefied Natural Gas (LNG) Fuelled Vessel. Available: https://www.seaspan.com/seaspan-ferries-corporation-announces-arrival-second-newliquefied-natural-gas-lng-fuelled-vessel

[22] A. Lakshmi. (2018, Dec 2018). Fjord1's First Battery-powered Ferry Named at Havyard. Available: https://www.marinelink.com/news/batterypowered-havyard437563

[23] (2019, March 2019). Stockholm's first hybrid ice-class passenger ferry. Available: https://www.danfoss.com/en/about-danfoss/news/dps/stockholm-s-first-hybrid-iceclass-passenger-ferry/ 
[24] (2018, March 2019). First electric ferry in Asia. Available: https://www.pressebox.com/pressrelease/baumueller-holding-gmbh-co-kg/Firstelectric-ferry-in-Asia/boxid/902539

[25] (2019, March 2019). Havyard ferries making the fjords greener. Available: https://www.havyard.com/news/2019/havyard-ferries-making-the-fjordsgreener/?fbclid=IwAR382fjBURmmpe2lRvTa6scDQ4qQJSZTYZBkNBOAFHk79xsblNGROmI0Ns

[26] P. Gunton. (2018, Dec 2018). FinFerries starts a quiet revolution. Available: https://www.passengership.info/news/view, finferries-starts-a-quietrevolution_50424.htm

[27] E. Gagatsi, T. Estrup, and A. Halatsis, "Exploring the Potentials of Electrical Waterborne Transport in Europe: The E-ferry Concept," Transportation Research Procedia, vol. 14, pp. 1571-1580, 2016.

[28] M. Holter and J. Hodges. (2018). The Next Ferry You Board Might Run on Batteries. Available: https://www.bloomberg.com/news/features/2018-03-13/the-next-ship-youboard-might-run-on-batteries

[29] (2018). CORVUS ENERGY SECURES CONTRACTS FOR FIVE ALL-ELECTRIC, BATTERY-POWERED FERRIES. Available: https://corvusenergy.com/corvus-energysecures-contracts-for-five-all-electric-battery-powered-ferries/

[30] M. Kane. (2018). Canada Gets Its First Fully Electric Vessels: 2 Massive Ferries. Available: https://insideevs.com/canada-first-fully-electric-vessels/

[31] J. Han, J.-F. Charpentier, and T. Tang, "An Energy Management System of a Fuel Cell/Battery Hybrid Boat," Energies, vol. 7, pp. 2799-2820, 2014.

[32] A. M. Bassam, A. B. Phillips, S. R. Turnock, and P. A. Wilson, "An improved energy management strategy for a hybrid fuel cell/battery passenger vessel," International Journal of Hydrogen Energy, vol. 41, pp. 22453-22464, 2016.

[33] A. M. Bassam, A. B. Phillips, S. R. Turnock, and P. A. Wilson, "Development of a multischeme energy management strategy for a hybrid fuel cell driven passenger ship," International Journal of Hydrogen Energy, vol. 42, pp. 623-635, 2017.

[34] M. Kalikatzarakis, R. Geertsma, E. Boonen, K. Visser, and R. Negenborn, "Ship energy management for hybrid propulsion and power supply with shore charging," Control Engineering Practice, vol. 76, pp. 133-154, 2018. 
[35] W. Lhomme and J. P. Trovão, "Zero-emission casting-off and docking maneuvers for series hybrid excursion ships," Energy Conversion and Management, vol. 184, pp. 427435, 2019.

[36] M. Othman, A. Anvari-Moghaddam, N. Ahamad, S. Chun-Lien, and J. M. Guerrero, "Scheduling of Power Generation in Hybrid Shipboard Microgrids with Energy Storage Systems," in 2018 IEEE International Conference on Environment and Electrical Engineering and 2018 IEEE Industrial and Commercial Power Systems Europe (EEEIC/I\&CPS Europe), 2018, pp. 1-6.

[37] C. Gamarra and J. M. Guerrero, "Computational optimization techniques applied to microgrids planning: A review," Renewable and Sustainable Energy Reviews, vol. 48, pp. 413-424, 2015.

[38] M. D. Al-Falahi, S. Jayasinghe, and H. Enshaei, "A review on recent size optimization methodologies for standalone solar and wind hybrid renewable energy system," Energy Conversion and Management, vol. 143, pp. 252-274, 2017.

[39] F. A. Khan, N. Pal, and S. H. Saeed, "Review of solar photovoltaic and wind hybrid energy systems for sizing strategies optimization techniques and cost analysis methodologies," Renewable and Sustainable Energy Reviews, vol. 92, pp. 937-947, 2018.

[40] A. Nabaei, M. Hamian, M. R. Parsaei, R. Safdari, T. Samad-Soltani, H. Zarrabi, et al., "Topologies and performance of intelligent algorithms: a comprehensive review," Artificial Intelligence Review, vol. 49, pp. 79-103, 2018.

[41] P. Kankanala, S. C. Srivastava, A. K. Srivastava, and N. N. Schulz, "Optimal control of voltage and power in a multi-zonal MVDC shipboard power system," IEEE Transactions on Power Systems, vol. 27, pp. 642-650, 2012.

[42] F. Shariatzadeh, C. B. Vellaithurai, S. S. Biswas, R. Zamora, and A. K. Srivastava, "Realtime implementation of intelligent reconfiguration algorithm for microgrid," IEEE Transactions on sustainable energy, vol. 5, pp. 598-607, 2014.

[43] E. A. Sciberras, B. Zahawi, D. J. Atkinson, A. Breijs, and J. H. van Vugt, "Managing shipboard energy: A stochastic approach special issue on marine systems electrification," IEEE Transactions on Transportation Electrification, vol. 2, pp. 538-546, 2016.

[44] F. Kanellos, "Optimal power management with GHG emissions limitation in all-electric ship power systems comprising energy storage systems," IEEE Transactions on Power Systems, vol. 29, pp. 330-339, 2014. 
[45] F. D. Kanellos, A. Anvari-Moghaddam, and J. M. Guerrero, "A cost-effective and emission-aware power management system for ships with integrated full electric propulsion," Electric Power Systems Research, vol. 150, pp. 63-75, 2017. 